\title{
Cell Sex and Sex Hormones Modulate Kidney Glucose and Glutamine Metabolism in Health and Diabetes
}

\author{
S Clotet-Freixas ${ }^{1,2}$, O Zaslaver ${ }^{3,4}$, C Pastrello ${ }^{5,6}$, M Kotlyar5,6, C McEvoy $^{1,2}$, S Farkona ${ }^{1,2}$, A Saha ${ }^{1,2}$, A \\ Boshart $^{1,2}$, M Chan $^{1,2}$, M Riera ${ }^{7}$, MJ Soler ${ }^{8}$, A Isenbrandt ${ }^{9,10}$, J Lamontagne-Proulx ${ }^{9,10}$, S Pradeloux ${ }^{9,10}, \mathrm{~K}$ \\ Coulombe $^{9}$, D Soulet ${ }^{9,10}$, AB Dart ${ }^{11,12}$, B Wicklow ${ }^{11,12}$, JM McGavock ${ }^{11,12}$, TD Blydt-Hansen ${ }^{13}$, I \\ Jurisica ${ }^{5,6,14,15}, \mathrm{M} \mathrm{Woo}^{1,16}$, JW Scholey ${ }^{17,18,19}, \mathrm{H}_{\text {Röst }}^{3,4}$, and A Konvalinka ${ }^{1,2,17,18,19}$
}

${ }^{1}$ Toronto General Hospital Research Institute, University Health Network, Toronto, ON, Canada ${ }^{2}$ Soham and Shaila Ajmera Family Transplant Centre, University Health Network, Toronto, ON, Canada ${ }^{3}$ The Donnelly Centre, University of Toronto, Toronto, ON, Canada ${ }^{4}$ Department of Molecular Genetics, University of Toronto, Toronto, ON, Canada ${ }^{5}$ Osteoarthritis Research Program, Division of Orthopedic Surgery, Schroeder Arthritis Institute, University Health Network, Toronto, ON, Canada

${ }^{6}$ Krembil Research Institute, University Health Network, Toronto, ON, Canada

${ }^{7}$ Kidney Research Group, Hospital del Mar Medical Research Institute, IMIM, Barcelona, Spain ${ }^{8}$ Hospital Universitari Vall d'Hebron, Division of Nephrology Autonomous University of Barcelona, Barcelona, Spain

${ }^{9}$ Neurosciences Axis, CHU de Quebec Research Center - Université Laval, Québec, QC, Canada

${ }^{10}$ Faculty of Pharmacy, Université Laval, Québec, QC, Canada

${ }^{11}$ Department of Pediatrics and Child Health, University of Manitoba, Winnipeg, MB, Canada.

${ }^{12}$ Diabetes Research Envisioned and Accomplished in Manitoba Research Team, Children's Hospital Research Institute of Manitoba, Winnipeg, MB, Canada.

${ }^{13}$ Department of Pediatrics, The University of British Columbia, Winnipeg, MB, Canada

${ }^{14}$ Departments of Medical Biophysics and Computer Science, University of Toronto, Toronto, ON, Canada

${ }^{15}$ Institute of Neuroimmunology, Slovak Academy of Sciences, Bratislava, Slovakia

${ }^{16}$ Department of Medicine, Division of Endocrinology, University Health Network, University of Toronto, Toronto, ON, Canada

17Department of Medicine, Division of Nephrology, University Health Network, Toronto, Canada

${ }^{18}$ Department of Laboratory Medicine and Pathobiology, University of Toronto, Toronto, Canada

${ }^{19}$ Institute of Medical Science, University of Toronto, Toronto, Canada

Word count: 12,023

\section{Corresponding author:}

Sergi Clotet-Freixas, $\mathrm{PhD}$

ORCID ID (Sergi Clotet-Freixas): $\underline{0000-0002-6965-4403}$

Department of Medicine, Division of Nephrology, University Health Network

585 University Avenue, room 11-PMB-189. M5G 2N2.Toronto, ON, Canada.

Telephone number: +1 (416)340-4800 ext. 6950. Fax: 18882478594

Sergi.ClotetFreixas@uhnresearch.ca 


\section{ABSTRACT}

Male sex is a risk factor for progression of diabetic kidney disease, but the reasons for this predilection are unclear. Here, we demonstrate that cell sex and sex hormones alter the metabolic phenotype of human proximal tubular epithelial cells (PTECs). Male PTECs displayed increased glycolysis, mitochondrial respiration, oxidative stress, apoptosis, and high glucose-induced injury, compared to female PTECs. This phenotype was enhanced by dihydrotestosterone (DHT) and linked to increased mitochondrial utilization of glucose and glutamine. Studies in vivo pointed towards increased glutamine anaplerosis in diabetic male kidneys. Male sex was linked to increased levels of glutamate, TCA cycle, and glutathione cycle metabolites, in PTECs and in the blood metabolome of healthy youth and youth with type 2 diabetes. Conversely, female PTECs displayed increased levels of pyruvate, glutamyl-cysteine, cysteinylglycine, and a higher GSH/GSSG ratio than male PTECs, indicative of enhanced redox homeostasis. Finally, we identified transcriptional mechanisms that control kidney metabolism in a sex-specific fashion. While X-linked demethylase KDM6A facilitated metabolic homeostasis in female PTECs, transcription factor HNF4A mediated the deleterious effects of DHT in male PTECs. This work uncovers the role of sex in glucose/glutamine metabolism, that may explain the roots of sex dimorphism in the healthy and diabetic kidney. 


\section{CONFLICT OF INTEREST STATEMENT}

The authors of this manuscript have conflicts of interest to disclose. Dr. Igor Jurisica reports receiving personal fees from Canadian Rheumatology Association, grants and nonfinancial support from IBM, and personal fees from Novartis, outside the submitted work. Dr. María José Soler reports honorarium for conferences, consulting fees and/or advisory boards from Astra Zeneca, NovoNordsik, Esteve, Vifor, Bayer, Mundipharma, Ingelheim Lilly, Jansen, ICU Medical, and Boehringer. All other authors have declared that no conflict of interest exists. 


\section{INTRODUCTION}

Chronic kidney disease (CKD) affects $>14 \%$ of the adult population and causes significant morbidity and mortality ${ }^{1}$. Diabetes is the leading cause of CKD worldwide, and diabetic kidney disease (DKD) is responsible for $\sim 40 \%$ of $C K D^{2}$. Importantly, the cumulative incidence of DKD after 30 years of diabetes is higher in men $(4.1 \%)$, compared to women $(2.5 \%)^{3}$. Moreover, diabetic men are at higher risk than diabetic women of progressing to end-stage kidney disease, with hazard ratios ranging from $2 \cdot 5-3 \cdot 0^{3-5}$. Studies involving patients with type 1 or type 2 diabetes and different follow-up times (2.5-18 years) have shown that male sex is significantly associated with the development of microalbuminuria and macroalbuminuria ${ }^{5-10}$. However, the reasons for the sex-based nature of DKD are incompletely understood. Both clinical and animal model studies predominantly include male subjects, leaving a major gap in the understanding of the role of sex or sex hormones in kidney physiology and in DKD progression ${ }^{11}$. A better understanding of this sex dimorphism will lead to the development of sex-specific predictive tools and treatments for bridging this critical gap in knowledge.

Proximal tubular epithelial cells (PTECs) are the most abundant cell type in the kidney. These cells are metabolically active, as they require high amounts of energy to facilitate renal sodium reabsorption ${ }^{12}$. In healthy PTECs, glucose is mainly converted to pyruvate for utilization by the tricarboxylic acid (TCA) cycle, the most efficient mechanism of energy production in the form of ATP $^{13}$. Glutamine participates in the antioxidant glutathione cycle ${ }^{14}$. Alterations in glucose and glutamine homeostasis are key contributors to the progression of $\mathrm{DKD}^{15}$. Increased glucose uptake triggers glycolysis and glutamine anaplerosis, resembling the Warburg effect ${ }^{15,16}$, which is increasingly recognized in $\mathrm{DKD}^{17,18}$. Aberrant glucose and glutamine metabolism also activates the hexosamine pathway, leading to hypertrophy and fibrosis ${ }^{19,20}$. In PTECs, these metabolic 
alterations are linked to the development of fibrosis ${ }^{21-2324,25}$. The importance of PTECs and their metabolism in DKD is supported by the remarkable effectiveness of SGLT2 inhibitors ${ }^{26-30}$.

We and others have demonstrated that functional and metabolic alterations in DKD develop in a sex-specific fashion ${ }^{31-42}$. Sex chromosomes define physiological features in adult somatic tissues $^{43}$, and influence the development of cardiovascular disease ${ }^{44}$, but little is known about the effect of cell sex on kidney metabolism and disease. In diabetic mice, male sex was linked to the development of severe kidney lesions, which were attenuated in the absence of androgens ${ }^{32,34}$. We demonstrated that the androgen dihydrotestosterone (DHT), but not the estrogen estradiol (EST), increased enzymes involved in glucose and glutamine metabolism in PTECs. These changes were confirmed in healthy and diabetic male mice and related to kidney injury ${ }^{31}$. We thus postulated that energy metabolism could be the key to understand sex hormone-related effects in kidney physiology and pathophysiology.

In this study, we investigate, for the first time, the effect of cell sex on kidney metabolism. We demonstrate that male sex and androgens confer a 'pro-oxidant' metabolic state and accelerate high glucose-induced mitochondrial injury in PTECs. In contrast, female PTECs show decreased oxidative stress and cell death, and preserved mitochondrial function upon exposure to high glucose. We also show increased intracellular levels of glutamate, TCA, and glutathione cycle metabolites in male PTECs, while female PTECs display increased levels of pyruvate. These sexspecific differences in metabolite levels in PTECs were reproduced in adolescents with and without diabetes. At the mechanistic level, we identify hepatocyte nuclear factor 4 alpha (HNF4A), a regulatory partner of androgen receptor $(A R)$ in the kidney ${ }^{45}$, as a key transcriptional regulator of glucose- and glutamine-related enzymes upregulated by androgens. We also identify X-linked lysine demethylase KDM6A as the key regulator of metabolism in the female kidney. We demonstrate that HNF4A mediates androgen-induced metabolic injury in male PTECs, while 
KDM6A contributes to the preservation of mitochondrial function in female PTECs. Overall, we identify a compelling link between glucose/glutamine metabolism and sex, that may explain the roots of sex dimorphism in the healthy kidney and in DKD.

\section{RESULTS}

\section{Male PTECs have a more energetic and oxidative phenotype compared to female PTECs}

We first investigated if sex hormones and the sex of the cell influence the metabolic phenotype of PTECs. Commercially available primary PTECs from 4 male and 5 female healthy donors of comparable ages were examined at baseline and after stimulation with sex hormones (Fig.1A).

Male PTECs showed significantly higher glycolysis and oxygen consumption rate (OCR) than female PTECs, both at baseline and after metabolic stress (Fig.1B). We next calculated the relevant metabolic parameters from glycolysis and OCR curves (Fig.S1). Together with a significant increase in baseline glycolysis (Fig.1C), male PTECs showed a modest increment in their maximal glycolytic capacity (Fig.1D) and glycolytic reserve (Fig.1E), compared to female cells. In addition, male PTECs displayed modestly increased basal respiration (Fig.1F) and ATPlinked respiration (Fig.1G), and a significantly increased maximal respiratory capacity (Fig.1H), and reserve capacity (Fig.1I), compared to female cells. No differences between sexes were found in proton leak (Fig.1J) or non-mitochondrial respiration (Fig.1K). In conjunction with their enhanced glycolytic response, male PTECs showed a numerically higher capacity for glucose uptake, relative to female PTECs (Fig.1L).

\section{The metabolic effects of male sex and dihydrotestosterone are linked to increased cell injury in PTECs}

We next investigated the potential role of sex hormones on the metabolic function of PTECs. In male PTECs, DHT significantly increased glycolysis and OCR at baseline, compared to CONT- 
and EST-treated cells. In female PTECs, both DHT and EST induced a significant increase in glycolysis and OCR at baseline and after metabolic stress, compared to CONT-treated cells (Fig.2A,B). Despite the significant effects of DHT and EST on the glycolytic activity of PTECs at baseline, none of the sex hormones significantly altered maximal glycolytic capacity or glycolytic reserve (Fig.S2A-C). In contrast, DHT significantly increased not only the basal respiration rate of male PTECs (Fig.S2D), but also their non-mitochondrial respiration (Fig.S2E). In female PTECs, the significant increase in OCR at baseline induced by DHT and EST, was accompanied by a significantly higher ATP-linked respiration (Fig.S2F), compared to CONT-treated cells.

We next examined if the functional differences linked to cell sex and sex hormones in PTECs related to a different propensity to consume glucose. We did not observe any significant differences in glucose levels in the media, at 16h (Fig.2C). However, at $24 \mathrm{~h}$ and $48 \mathrm{~h}$, all groups of male PTECs displayed a significant reduction in the extracellular levels of glucose (indicating increased consumption), compared to female PTECs receiving the same treatment (Fig.2D-E). Moreover, DHT significantly enhanced the glucose consumption in male and female PTECs, compared to CONT and EST treatments (Fig.2D,E).

In metabolic disorders such as DKD, excessive glucose consumption and utilization in the TCA cycle may result in mitochondrial stress and cell death ${ }^{46}$. We thus evaluated if the observed changes in metabolic function and glucose consumption induced by cell sex and sex hormones in PTECs were related to altered oxidative stress and apoptosis ${ }^{15,20,47}$. Importantly, increased glucose and oxygen consumption in male PTECs were accompanied by significantly higher levels of oxidative stress (Fig.2F) and early apoptosis (Fig.2G), compared to female cells. DHT-treated male PTECs showed significantly higher oxidative stress levels than CONT-treated cells (Fig.2F), as well as significantly augmented early apoptosis compared to EST-treated cells (Fig.2G). Despite increasing OCR in male PTECs and altering the metabolic function of female PTECs 
(Fig.2A,B), EST treatment did not increase oxidative stress or apoptosis, regardless of the cell $\operatorname{sex}(\mathbf{F i g} . \mathbf{2 F}, \mathbf{G})$.

\section{Androgen receptor mediates metabolic perturbations in male PTECs}

DHT had a dominant effect on glucose consumption in both male and female PTECs, and promoted glycolysis, oxidative stress, and apoptosis in male PTECs (Fig.2). To elucidate if the metabolic actions of DHT were mediated by AR, we exposed male and female PTECs to antiandrogen treatment for $2 \mathrm{~h}$ prior to sex hormone stimulation. Two different AR inhibitors with distinct mechanisms of action were employed: flutamide ${ }^{48}$ and enzalutamide ${ }^{49}$ (Fig.S3A). Pretreatment for $2 \mathrm{~h}$ with either FLUT $(1 \mu \mathrm{M})$ or ENZ $(1 \mu \mathrm{M})$ significantly prevented the DHT-induced increase in glycolysis (Fig.S3B, D-F, I) and mitochondrial respiration (Fig.S3C, G-I) in male PTECs and, to a lesser extent, in female PTECs. Pre-treatment with FLUT also numerically reduced superoxide ion levels in DHT-treated male and female PTECs. A similar trend was observed in DHT-treated male and female PTECs pre-treated with ENZ (Fig.S3J). Overall, the effects of AR inhibition were greater in male PTECs than in female PTECs. This sex dimorphism could be explained by increased AR expression in male PTECs (Fig.S3K). These data suggest that DHT-induced metabolic alterations in PTECs are mediated by AR.

\section{High glucose-induced mitochondrial dysfunction and ATP depletion are accelerated in male PTECs}

We next investigated if high glucose is a modifier of the identified sex differences in the metabolism of PTECs. Male and female PTECs were exposed to high glucose for $2 \mathrm{~h}$ (mimicking acute hyperglycemia), and 48h-96h (chronic hyperglycemia).

In male PTECs, high glucose exposure resulted in a marked increase in glycolysis and mitochondrial respiration at $2 \mathrm{~h}$ (Fig.3A). While the gradual increase in mitochondrial respiration persisted after $48 \mathrm{~h}$ of hyperglycemia, the basal glycolytic rate of male PTECs started to decline 
at this time point. After $96 \mathrm{~h}$ of exposure to high glucose, male PTECs displayed a reduction in both glycolysis and OCR, as compared to the previous time point. In female PTECs, $2 \mathrm{~h}$ of hyperglycemia was also associated with a significant increase in glycolysis and OCR. Interestingly, both parameters were further increased at $48 \mathrm{~h}$, especially after metabolic stress. At 96h, high glucose induced a more pronounced increase in OCR in female PTECs, but a reduction in the glycolytic rate, as compared to the previous time points (Fig.3A).

The differences between male and female PTECs exposed to high glucose over time were reflected in glycolysis- and OCR-related metabolic parameters. After $2 \mathrm{~h}$ and $48 \mathrm{~h}$ of high glucose, male PTECs still showed a higher baseline glycolysis (Fig.3B), maximal glycolytic capacity (Fig.3C), glycolytic reserve (Fig.3D), basal respiration (Fig.3E), maximal respiratory capacity (Fig.3F), ATP-linked respiration (Fig.3G), reserve respiratory capacity (Fig.3H) and nonmitochondrial respiration (Fig.3I) than female PTECs. This effect was not observed after 96h of high glucose exposure. At this time point, the maximal respiratory capacity (Fig.3F) and reserve respiratory capacity (Fig.3H) were significantly higher in female, compared to male PTECs.

Our findings suggest that high glucose conditions elicit sex-specific metabolic changes in PTECs, that are time-dependent. Male PTECs displayed a more energetic phenotype during acute exposure (2h), and a switch toward a more quiescent state at $48 \mathrm{~h}$, with a clear reduction in their glycolytic rate and mitochondrial activity at $96 \mathrm{~h}$. This slowing of glycolytic and oxygen consumption rates was delayed in female PTECs, which showed a more energetic phenotype after $48 \mathrm{~h}$ of high glucose, and a change towards a more oxidative phenotype at 96h (Fig.3J). High glucose induced a decline in the intracellular levels of ATP over time. Again, this effect was more pronounced in male PTECs, which showed the lowest ATP levels at 96h (Fig.3K). These data suggest that male sex accelerates high glucose-induced reduction in glycolysis and OCR, and subsequent ATP depletion in PTECs. 
We also investigated if co-treatment with sex hormones modified the effects of high glucose on PTEC metabolic function (Fig.S4). DHT-treated PTECs displayed the highest levels of ATP and oxidative stress after $96 \mathrm{~h}$ of high glucose, and this effect was more pronounced in male compared to female PTECs. In line with their reduced respiration, EST-treated male PTECs exposed to high glucose showed lower levels of ATP and superoxide ion, compared to CONT- and DHT-treated cells (Fig.S4J,K). These data suggest that EST accelerates the decline in mitochondrial function induced by high glucose in male PTECs, while delaying this effect in female PTECs. In contrast, DHT promotes oxidative stress in the setting of high glucose, particularly in male PTECs.

\section{Increased mitochondrial utilization of glucose and glutamine contributes to the enhanced metabolic activity of male PTECs}

Early alterations in the metabolism of glucose, glutamine and pyruvate are key contributors to $\mathrm{DKD}^{15,20}$. We therefore deciphered the specific contribution of these substrates to the metabolic phenotype of PTECs. We subjected male and female PTECs to nutrient restricted conditions, prior to the measurement of glycolysis and OCR. As observed under non-restricting conditions (Fig.1), exposure to glucose alone resulted in a similar extent of increased respiration and glycolysis in male compared to female cells (Fig.4A-C). Importantly, when glutamine was the only substrate present in the media, male PTECs showed the greatest increase in their maximal respiratory capacity and respiratory reserve, compared to female PTECs (Fig.4D-F). In contrast, no marked sex differences in OCR and glycolysis were observed in the presence of pyruvate (Fig.4G-I).

Our substrate-restricting studies suggest a higher mitochondrial utilization of glucose and glutamine by male PTECs, compared to female PTECs. In turn, female PTECs showed a relative preference for pyruvate utilization over glucose and glutamine. In male PTECs, glucose seems to be more rapidly metabolized through glycolysis, and consequently, glutamine may be supporting the mitochondrial TCA cycle to a higher extent than in female PTECs. 


\section{Male sex is linked to increased circulating levels of glucose, lactate, glutamine, and glutamate in diabetic and healthy mice}

Metabolites are considered the final output of the cell biological activity and reflect the metabolic states of a living system ${ }^{50}$. Whereas lactate is the end-product of glucose utilization through glycolysis, glutamate is the product of glutaminolysis and a key intermediate for the entry of glutamine-derived carbons to the TCA cycle in the mitochondria, after further conversion to alphaketoglutarate ( $\alpha-K G$ ) (Fig.5A $)^{51,52}$. We next examined in vivo the circulating and urinary levels of glucose, glutamine, and their breakdown products (lactate and glutamate).

We first measured these 4 metabolites in the plasma of control and type 1 diabetic (T1DM) male and female Akita mice. At 16 weeks of age, Akita mice show microalbuminuria and glomerular enlargement, indicative of early $\mathrm{DKD}^{53}$. We examined sexually mature animals at two different ages: 8 weeks and 16 weeks $^{54}$ (Fig.5B). Hyperglycemia in diabetic males was associated with increased lactate levels at 8 weeks, but not at 16 weeks of age. Diabetic females showed no changes in plasma lactate levels, compared to healthy animals. All diabetic groups showed a clear increase in the plasma levels of glutamine and a significant reduction in glutamate, compared to their healthy controls, and regardless of sex and age (Fig.5C,D).

Male sex was associated with increased circulating levels of glucose, lactate, glutamine, and glutamate. This effect was modified by age and diabetes. The circulating levels of lactate and glutamate were significantly higher in 16-week-old healthy males, compared to females. In contrast, within the diabetic groups, sex differences in the plasma levels of glucose, lactate, glutamine, and glutamate were more evident at 8 weeks than at 16 weeks (Fig.5C,D). Interestingly, male mice showed lower urinary excretion of glutamine and glutamate than female mice, and this decrease was prevented by androgen reduction through gonadectomy 
(Fig.S5A,B). These data suggest that male sex and androgens promote the utilization of glutamate by kidney cells, rather than its excretion.

The link between male sex and increased circulating levels of glucose, lactate, and glutamate is conserved in humans

To test the translational potential of our findings, we investigated the effects of sex and diabetes on the serum metabolome of 10-18 years old adolescents (iCARE cohort) ${ }^{55}$ with early onset type 2 diabetes (T2DM, $\mathrm{n}=159)$, and their age- and body weight-matched controls without diabetes $(\mathrm{n}=45)$ (Fig.5E). This cohort was particularly relevant, as our findings in vivo supported sexspecific effects early in diabetes. The median duration of diabetes was 2.3 years and was comparable between male and female patients (Table 1). On average, patients with T2DM displayed a significant but minimal increase in urine albumin-to-creatinine ratio (median 0.7 $\mathrm{mg} / \mathrm{mmol}$ ), compared to controls without T2DM (median $0.2 \mathrm{mg} / \mathrm{mmol}$ ). Hypertension was present in $50 \%$ of patients with T2DM and $40 \%$ of controls. Patients with T2DM showed an increase in eGFR $\left(143.1 \mathrm{ml} / \mathrm{min} / 1.73 \mathrm{~m}^{2}\right.$ vs $\left.139.4 \mathrm{ml} / \mathrm{min} / 1.73 \mathrm{~m}^{2}\right)$, indicative of early hyperfiltration. This eGFR increase was more marked in males with T2DM (median $154.8 \mathrm{ml} / \mathrm{min} / 1.73 \mathrm{~m}^{2}$ ) compared to females (median $139.4 \mathrm{ml} / \mathrm{min} / 1.73 \mathrm{~m}^{2}$ ). Overall, these clinical features are indicative of early stages of DKD among both male and female patients with T2DM.

Supporting our findings in vivo, patients with T2DM displayed increased serum levels of glucose and lactate, and decreased levels of glutamine, in comparison to the control individuals (Fig.5F, Table S1). In agreement with our experimental observations, male adolescents without T2DM displayed significantly increased levels of glucose, lactate, and glutamate, compared to females without T2DM. This increase was also observed in males with T2DM, relative to females with T2DM, without reaching statistical significance. Interaction analyses confirmed the independent contribution of sex and diabetes to the levels of each metabolite. While diabetes was an 
independent modifier of the circulating levels of glucose and lactate, glutamate levels were exclusively modified by sex $(P=0.031$, Table S2).

TCA and glutathione cycle metabolites arising from glutamate are increased in male PTECs and in male individuals

Our data suggested that glutamine and glutamate were involved in major metabolic sex differences. We next examined the expression of key glutamine/glutamate transporters and enzymes. We measured in vivo the renal levels of Slc38a3 (important glutamine importer), Gls (glutaminase, which converts glutamine to glutamate), Glud1 (glutamate dehydrogenase, which converts glutamate to $\alpha-K G$ ), and Oxgr1 (G protein-coupled receptor that mediates signaling induced by $\alpha-K G$, that is not used up in the TCA cycle) (Fig.S5C). Under non-diabetic conditions, female sex was associated with increased kidney expression of all four genes. In turn, hyperglycemia was linked to a significant increase in the kidney levels of Slc38a3 and Glud1 exclusively in diabetic male mice. Diabetes and male sex were also associated with the lowest kidney expression of Oxgr1 (Fig.S5D). These data suggest a higher conversion of glutamine to glutamate in females, but increased utilization of $\alpha-K G$ in the TCA cycle by diabetic and healthy male kidneys, compared to female kidneys.

In addition to being a carbon source for the TCA cycle, glutamate is also a major substrate of the anti-oxidant glutathione cycle ${ }^{14}$. To determine if glutamate-derived metabolites of the TCA and glutathione cycles were altered between male and female PTECs, we analysed the intracellular metabolites of male and female PTECs exposed to normal glucose (Table S4A,B). Supporting our findings in animals and humans, male PTECs displayed a significant increase in the intracellular levels of glutamate, compared to female PTECs $(Q<0.05)$ (Fig.6A). Importantly, male sex was linked to an increase in the intracellular levels of 7 metabolites of the TCA cycle, which represent the 'pro-oxidant' metabolism of glutamate. Specifically, male PTECs displayed a significant increase in the intracellular levels of citrate, aspartate, and malate $(Q<0.05)$, and a 
numerical increase in the intracellular levels of isocitrate, $\alpha-K G$, succinate, and fumarate, in comparison to female PTECs. Female PTECs showed a marked increase in the intracellular levels of pyruvate $(Q<0.001)$, relative to male PTECs (Table S4C, Fig.6A). We also identified sex differences in the intracellular metabolites of the glutathione cycle, the 'antioxidant arm' of glutamate metabolism. To exert their antioxidant function, some GSH molecules (the reduced form of glutathione) exit the cycle and neutralize the negative charge of reactive oxygen species, while generating GSSG (oxidized form of glutathione) as by-product ${ }^{56}$. Male PTECs showed significantly higher intracellular levels of GSH and GSSG $(Q<0.001)$, compared to female PTECs. In contrast, female PTECs displayed a significant increase in the GSH/GSSG ratio $(Q<0.05)$, an indicator of adequate redox balance ${ }^{57}$. Female cells also displayed significantly increased levels of glutamyl-cysteine $(Q<0.001$, precursor of $G S H)$ and cysteinylglycine $(Q<0.01$, product of $G S H)$. While the cycling of glutathione metabolites seems to be more maintained in female PTECs, a higher efflux of GSH may be occurring in male PTECs, indicating a higher demand for antioxidant mechanisms (Table S4C, Fig.6A).

Our main findings at the cell metabolome level were reproduced in the iCARE cohort. Females with and without T2DM had numerically increased circulating levels of pyruvate, compared to males. In contrast, male sex was associated with increased circulating levels of TCA cycle metabolites (namely succinate, fumarate, and malate). This increase relative to females was significant only among youth without T2DM, but a similar pattern was observed among patients with T2DM (Fig.6B). We also identified $17 \gamma$-glutamyl-aminoacids, which play a key role as substrates of the glutathione cycle ${ }^{58}$, that were increased in the serum of male individuals (Table S1, Figure 6C,D). Among them, circulating levels of $\gamma$-glutamyl-epsilon-lysine, $\gamma$-glutamyl-alanine, $\gamma$-glutamyl-alpha-lysine, $\gamma$-glutamylleucine,$\quad \gamma$-glutamylglutamate, $\gamma$-glutamylisoleucine, and $\gamma$ glutamylmethionine were significantly increased in healthy male individuals, compared to females, and the same trend was observed among patients with T2DM. Most of these metabolites 
(11/17 in males and 13/17 in females) were also significantly decreased by diabetes (Table S1,

Figure 6C,D). These $17 \gamma$-glutamyl-aminoacids strongly and positively correlated with each other, reinforcing their coregulation (Fig.S6A, Table S3). Subsequent interaction analyses showed that sex was an independent modifier of circulating levels of succinate $(P=0.034)$. Both sex and diabetes were independent modifiers of the levels of $\gamma$-glutamyl-a-lysine and $\gamma$ glutamylmethionine, while the interaction between the two factors was a modifier of $\gamma$-glutamyl-Elysine levels (Table S2). Interestingly, the circulating levels of $15 / 17 \gamma$-glutamyl-aminoacids significantly and negatively correlated with the levels of glutamine $(Q<0.05)$, while all $17 \gamma$ glutamyl-amino acids significantly and positively correlated with the circulating levels of glutamate. Only 3/17 $\gamma$-glutamyl-amino acids correlated with the levels of glucose (Fig.S6B, Table S3). These observations suggest that sex differences in the levels of $\gamma$-glutamyl-amino acid metabolites in the blood may be due to a sex-specific metabolism of glutamine and glutamate by male and female tissues.

The formation of $\gamma$-glutamyl-amino acids is based on the conjugation of a glutamate molecule to itself or to another amino acid, via $\gamma$-glutamyl bonds ${ }^{59,60}$. In addition to glutamate, ten amino acids can be a source of carbons for the TCA cycle in the tissues ${ }^{61}$. We postulated that increased kidney conjugation of these amino acids would be reflected in their decreased urine excretion. Urine levels of glycine were significantly increased in females with or without T2DM, as compared to males $(P<0.001$, Fig.6D). Significant interaction was confirmed between sex and urinary levels of glycine (Table S2). Females with or without T2DM also displayed increased urinary excretion of isoleucine, methionine, valine, serine, and threonine, compared to males (Fig.6D). It is noteworthy that the pattern of these 6 amino acids in the urine was a mirror-image of the corresponding $\gamma$ glutamyl-amino acid levels in the circulation. A higher demand for amino acids as metabolic substrates in the human male kidney, relative to the female kidney, could explain this effect. 


\section{KDM6A orchestrates metabolic homeostasis in female PTECs}

Our next goal was to identify transcriptional regulators of sex-specific metabolic changes in PTECs. We studied genes encoding the proteins upregulated by sex hormones in PTECs ${ }^{31}$. To identify regulators that may underpin the sex chromosome and sex hormone effects, we analyzed 4 publicly available gene datasets of human healthy kidneys (NephroSeq) ${ }^{62-64}$. We identified 196 genes increased in male kidneys and 139 genes increased in female kidneys (Fig.S7, Table S5), and used these genes to computationally predict their transcriptional regulators (see Supplemental Methods).

We first focused on the datasets linked to female sex hormones. We identified 49 regulators significantly enriched among the 18 proteins increased by EST, but not by DHT. We also identified 339 regulators significantly enriched exclusively among genes upregulated in the female kidney, but not in the male kidney (Table S6, Fig.7A). Among these 339 regulators, we identified the Xlinked lysine demethylase KDM6A, also known as UTX, which was increased at transcriptional level in the female kidney (Fig.7B). The main function of KDM6A is to activate gene expression by removing two methyl groups from H3K27me3, a repressive mark on DNA histones ${ }^{65,66}$. KDM6A mediates proliferation and metabolism, and influences glutamine demands, as it requires $\alpha-K G$ to function ${ }^{67,68}$.

Little is known about the role of KDM6A in the kidney, and whether such role is sex-specific. In this study, KDM6A was predicted to regulate 22 genes increased in the female kidney $(Q<0.05)$. Five of these genes (CCNL1, FOXN3, IFRD1, PRIM2, and WNK1) are involved in cell cycle control and proliferation ${ }^{69}$. Together with two additional X-linked regulators (ZFX and DDX3X), KDM6A was predicted to participate in the regulation of the mitochondrial pyruvate dehydrogenase gene PDHB (Fig.7C). KDM6A, ZFX, DDX3X, and PDHB genes were increased in the human female kidney, relative to the male kidney (Table S5). KDM6A expression was also significantly increased in female compared to male PTECs (Fig.7D). Female mice had 
significantly increased kidney gene levels of Kdm6a compared to males, even after ovariectomy (removal of estrogens) (Fig.7E).

Given the conserved sex-biased expression of renal KDM6A, and its link to metabolism, we investigated the role of KDM6A in male and female PTECs. Pharmacological inhibition of KDM6A impaired metabolic function and viability of PTECs in a sex-specific fashion (Fig.7F-P). Treatment with the prototypic KDM6A inhibitor GSK-J4 ${ }^{70,71}$, but not the inactive control GSK-J5, decreased mitochondrial function (Fig.7F) and increased glycolysis (Fig.7G), in female PTECs. Interestingly, KDM6A inhibition in female PTECs reduced their maximal respiratory capacity (Fig. 7I) and significantly decreased their respiratory reserve (Fig.7J), without affecting ATP-linked respiration (Fig.7K). KDM6A inhibition in female PTECs also resulted in a significantly increased basal glycolytic rate (Fig.7L) and a higher maximal glycolytic capacity (Fig.7M). A metabolic shift toward reduced mitochondrial respiration and increased glycolysis may result in a decreased capacity to generate ATP, compromising proliferation and viability ${ }^{72}$. Increased glycolysis in female PTECs exposed to GSK-J4 was accompanied by a significant decrease in intracellular ATP (Fig.7N) and cell viability (Fig.70,P), elevated levels of superoxide ion (Fig.7Q) and a significant increase in cell death (Fig.7R). KDM6A may maintain metabolic homeostasis in female PTECs, helping them prioritize the mitochondrial use of energy substrates over glycolysis. In male PTECs, KMD6A inhibition significantly increased basal respiration (Fig.7H), decreased cell viability (Fig.7N-P), and increased cell death (Fig.7R). However, these changes were not linked to altered glycolysis in male PTECs (Fig.7G, L, M). 


\section{HNF4A mediates the deleterious effects of androgens in male PTECs}

We next examined transcriptional regulators linked to male sex and sex hormones in the kidney. We identified 343 factors significantly enriched among the 60 proteins upregulated by DHT in male PTECs, but not among the proteins altered by EST. We also identified 608 factors enriched among the genes upregulated in the male kidney (Table S6, Fig.8A). We were particularly interested in hepatocyte nuclear factor 4 alpha (HNF4A), predicted to regulate DHT-induced proteins in male PTECs, and genes increased in the human male kidney (Fig.8B). HNF4A mediates fatty acid, glucose and glutamine metabolism ${ }^{73-77}$, and is considered a central regulator of the kidney cell metabolism ${ }^{78-80}$. HNF4A was predicted to regulate the expression of 6 enzymes that were upregulated by DHT in male PTECs ${ }^{31}$, and that play a critical role in the metabolism of glucose (GPI, TKT), glutamine/glutamate (GGH, GLUD1, GLS), or both (GNPNAT1). HNF4A was also predicted to regulate 6 transcription factors encoded on the $Y$ chromosome, either directly (ZFY, EIF1AY, RPS4Y1), or indirectly (UTY, DDX3Y, KDM5D) (Fig.8C). All 6 Y-linked regulators were exclusively expressed at the gene level in male human kidneys and/or male PTECs (Table S5, Fig.8B,C, Fig.S8).

In the kidney, HNF4A has been proposed as the main partner of $\mathrm{AR}^{45}$. Along the same lines, $\mathrm{DHT}$ treatment significantly increased HNF4A gene expression in both male and female PTECs (Fig.8D). Similarly, kidney Hnf4a gene expression was significantly increased in male compared to female mice, and this increase was completely prevented in gonadectomized males (Fig.8E). In contrast, HNF4A gene expression did not differ between human male and female healthy kidneys, in publicly available datasets (Table S5). In summary, HNF4A was modified by male sex hormones, but not sex. We wondered if HNF4A also mediated the metabolic effects of DHT. We thus inhibited HNF4A in DHT-treated male and female PTECs, using BI6015, a pharmacological antagonist of HNF4A ${ }^{81}$. Co-treatment with BI6015 significantly prevented the DHT-induced increase in mitochondrial respiration, glycolysis, and apoptosis, and this effect was more 
pronounced in male PTECs (Fig.8F-O). HNF4A inhibition decreased basal respiration (Fig.8H), maximal respiratory capacity (Fig.8I), respiratory reserve (Fig.8J), ATP-linked respiration (Fig.8K), maximal glycolytic capacity (Fig.8M), and glycolytic reserve (Fig.8N) of DHT-treated male PTECs. These metabolic effects were associated with a decrease in apoptosis (Fig.80). BI6015 also reduced these parameters in female PTECs exposed to DHT, but in a more subtle fashion (Fig.8H-O). Interestingly, HNF4A inhibition significantly reduced the cellular ATP levels in DHT-treated PTECs, but also in control-treated cells, suggesting that HNF4A also governs ATPproducing mechanisms in PTECs independently from androgens (Fig.8P). These findings suggest that HNF4A mediates the deleterious metabolic effects of androgens, but also has androgen-independent metabolic effects, in male PTECs. 


\section{DISCUSSION}

The existence and clinical implications of the sex-based progression of DKD are well recognized ${ }^{82-85}$. Despite its importance, the underlying causes of the sex dimorphism in health or in disease remain unknown. Here, we demonstrate that: 1) male sex and DHT promote oxidative metabolism and cell injury in PTECs, while female sex promotes a more quiescent metabolism; 2) male PTECs are more susceptible to high glucose-induced injury and display a higher mitochondrial utilization of glucose and glutamine, while female PTECs show a predilection for pyruvate; 3) male sex is linked to increased levels of lactate, glutamate, TCA cycle, and glutathione cycle metabolites, while female sex is linked to increased levels of pyruvate, in vitro, in vivo and in healthy and diabetic individuals; and 4) KDM6A orchestrates metabolic homeostasis in female PTECs, while HNF4A mediates the deleterious effects of androgens in male PTECs. This study supports our previous proteomics findings ${ }^{31}$, and identifies, for the first time, a compelling link between glucose/glutamine metabolism and sex, that may explain the roots of sex dimorphism in DKD and possibly other kidney diseases.

Growing evidence demonstrates that the contribution of sex chromosomes in health and disease is broader than previously thought ${ }^{86}$. Genes escaping from the inactivation of one of the $\mathrm{X}$ chromosomes can be elevated in adult female tissues ${ }^{65}$, while $\mathrm{Y}$ chromosome genes are expressed by adult, somatic male tissues ${ }^{87,88}$. In the kidney, genes encoded by sex chromosomes can exhibit sex-biased expression and exert important functions ${ }^{89-91}$. In mammals, both sex chromosomes and sex hormones control tissue morphogenesis, organ size and metabolism, at different stages of life $e^{92,93}$. These effects are evolutionary connected to the specific metabolic demands of male and female tissues ${ }^{86}$, which may result in a different susceptibility to metabolic diseases $^{92,94}$. In DKD, sex differences in progression have been linked to a differential activation of mechanisms controlling renal hemodynamics, such as the renin-angiotensin system ${ }^{32,33}$ or nitric oxide signaling ${ }^{33,95,96}$. We previously uncovered a link between androgens and increased 
expression of metabolic enzymes in PTECs, and validated our key findings in two models of diabetes $^{31}$. We now considered the sex of the kidney cell as a principal study variable. Our novel approach led to the identification of fundamental sex differences in the metabolism of kidney cells, incentivising a paradigm shift in the study of sex differences in kidney health and disease.

Our main observation at the functional level is that male sex and DHT induce a more energetic and oxidative metabolic phenotype in PTECs, based on increased glucose consumption, glycolysis, mitochondrial respiration, oxidative stress, and apoptosis. Supporting these findings, we and others have shown increased oxidative stress levels in the kidneys of non-diabetic and diabetic male compared to female animals ${ }^{31,97,98}$. Increased ROS production by male mitochondria has also been described in other tissues ${ }^{99-101}$. We have now demonstrated that this sex dimorphism is conserved in PTECs, and attributed to both cell sex and sex hormones. PTECs are the most abundant and energy-demanding cell type in the kidney and possess a high mitochondrial content ${ }^{102}$. Mitochondrial respiration is the main ATP-producing mechanism, but also results in the generation of oxidative stress ${ }^{103}$. One may postulate that the higher rates of mitochondrial respiration in male PTECs explain the augmented levels of oxidative stress in male kidneys. Therefore, identifying and targeting the metabolic mechanisms responsible for this mitochondrial hyperactivity may help diminish oxidative stress and DKD progression in males.

Increased mitochondrial utilization of glucose and glutamine by male PTECs may explain their increased rates of respiration. Although fatty acid oxidation (FAO) is considered the main energyproducing mechanism in proximal tubules ${ }^{104}$, mitochondrial glucose and glutamine utilization are also crucial for the kidney cell homeostasis ${ }^{105-107}$. In DKD, defective renal FAO is accompanied by increased glycolysis and altered glutamine metabolism ${ }^{108-110}$. A higher influx of glucose to kidney cells may compromise the balance between the mitochondrial and glycolytic fates of glucose, resulting in enhanced lactate secretion. This shift leads to increased demands for glutamine to maintain acid/base homeostasis and to sustain the TCA cycle, through conversion 
to glutamate ${ }^{15,20,47}$. The increase in glucose and glutamine utilization by the mitochondria of male PTECs may explain why male kidneys are more prone to DKD injury than female kidneys. Indeed, male PTECs exposed to high glucose showed an initial increase in glycolysis and oxygen consumption, which remained higher than in female cells. These changes were followed by a more rapid decline ATP levels, a sign of injury in kidney cells exposed to high glucose ${ }^{111,112}$. Increased glycolysis and enhanced mitochondrial utilization of glutamine in male PTECs could predispose them to a more severe injury under high-glucose conditions, when glycolysis is favoured $^{113,114}$.

Our approach revealed sex differences in glutamate-derived metabolites in PTECs that were conserved in a relevant clinical setting. Male PTECs showed increased levels of glutamate and 7 metabolites of the TCA cycle, the metabolic pillar of mitochondrial respiration ${ }^{115}$. Of those, glutamate, malate, succinate and fumarate were also increased in the blood metabolome of male subjects with and without T2DM. Krumsiek et al. identified increased levels of circulating malate in healthy males, compared to females ${ }^{116}$. In vivo, succinate levels were increased in the kidneys of male mice, compared to females, and changed in a sex-specific fashion in the setting of $\mathrm{T1DM}^{117}$. Although we did not measure the TCA cycle metabolites in the urine, a previous analysis of the healthy human urine metabolome showed that males had a lower excretion of malate, citrate, succinate, and fumarate than females ${ }^{118}$. Our findings point towards a higher activation of the TCA cycle in the male kidney, which could explain the reduced excretion of TCA metabolites in males ${ }^{118}$. Our sex-based observations regarding the TCA cycle may be important in DKD ${ }^{119-}$ ${ }^{123}$. For example, altered urine and plasma levels of glutamate, citrate, malate, and fumarate predicted CKD progression in T2DM, especially in male patients ${ }^{119,120,123}$. Increased TCA cycle metabolites in males may reflect hyperactivation of this pathway and impaired redox balance in kidney cells. 
Male sex was also linked to increased cellular levels of GSH and GSSG, the effector metabolites of the glutathione cycle ${ }^{56}$. In turn, female PTECs displayed elevated levels of glutamyl-cysteine and cysteinylglycine as well as elevated GSH/GSSG ratio (indicative of a favourable redox balance $\left.{ }^{57}\right)$. Supporting our findings, decreased oxidative stress in female tissues has been linked to higher antioxidant capacity, increased GSH/GSSG ratio, and differential activation of glutathione-related genes and enzymes under healthy ${ }^{99,100,124}$ and diabetic conditions ${ }^{125,126}$. A higher efflux of GSH may be occurring in male PTECs, since they displayed higher oxidative stress than female PTECs and may have a higher demand for antioxidant mechanisms. In the human cohort, we identified $17 \gamma$-glutamyl-aminoacids intimately linked to the glutathione cycle that were significantly increased by male sex and decreased by diabetes. Similarly, Krumsiek et al. found higher levels $\gamma$-glutamylleucine, $\gamma$-glutamylvaline, $\gamma$-glutamylphenylalanine, $\gamma$ glutamylisoleucine, $\gamma$-glutamyltyrosine in male compared to female sera ${ }^{116}$. Our work confirms the link between sex and circulating $\gamma$-glutamyl-aminoacids and demonstrates that this aspect of sex dimorphism is conserved in the setting of T2DM. In urine, we found a strong association between female sex and increased excretion of glycine. This association was observed before in healthy subjects $^{118}$, and we now describe it in T2DM. The urine excretions of isoleucine, methionine, valine, serine, and threonine were increased by female sex and also by diabetes, mirroring the pattern of their corresponding $\gamma$-glutamyl-aminoacids in the circulation. While glycine is a natural precursor of $\mathrm{GSH}$, all 6 aminoacids can conjugate to glutamate and facilitate its utilization in the glutathione cycle ${ }^{127,128}$. A higher demand for these aminoacids as substrates of the glutathione cycle in the male kidney, relative to the female kidney, could explain their increased excretion in females.

The different type of diabetes between the animals (T1DM) and humans (T2DM) studied here may represent a limitation. However, renal metabolic alterations in DKD are triggered by hyperglycemia and have been described in both T1DM and T2DM ${ }^{15,129}$. Furthermore, Akita mice 
show microalbuminuria and hyperfiltration ${ }^{53,130}$, resembling the renal function profile of the iCARE participants with T2DM. In contrast to prior metabolomics studies ${ }^{119-123}$, we identified metabolite changes that occurred early after the onset of diabetes, and before the establishment of overt DKD. The value of reporter metabolites that can infer alterations in tissue homeostasis in a noninvasive fashion, and at early stages of disease, is increasingly recognized in nephrology ${ }^{131-133}$.

At the mechanistic level, we identified a link between KDM6A and female kidney metabolism. KDM6A mediates epigenetic modifications ${ }^{67}$, which are linked to metabolism and "molecular memory" in $\mathrm{DKD}^{20,134}$. KDM6A can escape from $\mathrm{X}$ inactivation, resulting in increased expression due to a double gene dosage in female $(X X)$, compared to male tissues $(X Y)^{65,135,136124,125}$. Here we show increased KDM6A expression in female PTECs and mouse kidneys. KDM6A inhibition in female PTECs led to a metabolic shift characterized by increased glycolysis, oxidative stress, and apoptosis, suggesting that KDM6A contributes to maintenance of metabolic homeostasis. In agreement, studies in autoimmunity models revealed that KDM6A mediates metabolism and exerts sex-specific functions in macrophages and T cells ${ }^{68,136}$. Furthermore, female chromosomes and increased KDM6A exerted protective actions in mice with cardiac ischemia ${ }^{137}$. Our female PTECs showed higher levels of pyruvate than male PTECs. In turn, our bioinformatics analyses revealed that KDM6A participates in the regulation of PDHB. This enzyme catalyzes the mitochondrial use of pyruvate, preventing its conversion to lactate through glycolysis ${ }^{138}$. By lowering glycolysis, KDM6A may contribute to an increased cytosolic pool of pyruvate in female PTECs. This idea is supported by data generated in immune cells $s^{136,139,140}$. New lines of investigation of sex differences and epigenetics may help identify important sex-based regulatory mechanisms in kidney health and disease.

Finally, we have identified a link between androgens and HNF4A, a central regulator of glucose and glutamine metabolism ${ }^{73-77,141}$. In the kidney, HNF4A is highly expressed in PTECs ${ }^{142,143}$ and has been proposed as the main co-regulator of $A R^{144}$. Accordingly, androgens increased HNF4A 
levels in our PTECs, while orchidectomy decreased kidney Hnf4a. Our findings reinforce the link between sex hormones and HNF4A in the kidney. We have also demonstrated that the deleterious metabolic effects of androgens in male PTECs are mediated by HNF4A. At first glance, these findings may seem contradictory, as previous studies suggest that the renal metabolic actions of HNF4A are beneficial ${ }^{145,146}$. HNF4A also regulates gene expression via androgen-independent mechanisms ${ }^{143,145,147,148}$. While androgen-independent HNF4A actions seem essential for PTECs, androgen-dependent mechanisms of this transcription factor may result in mitochondrial hyperactivity and predispose to oxidative stress. In diabetes, high glucose alters the levels and activity of HNF4A ${ }^{149,150}$. In turn, HNF4A regulates genes altered in PTECs from diabetic patients ${ }^{151}$. Since both DHT and HNF4A control metabolic processes altered by diabetes, they may play a synergistic role in $\mathrm{DKD}^{31,73,152,153}$.

Our work sheds new light on the sex-based dimorphism in kidney metabolism and DKD. Our sexbalanced approach spearheads new research paradigms through the investigation of the distinct metabolic wiring of male and female kidney cells that can contribute to homeostasis and disease. We are the first to identify fundamental sex differences in the kidney cell metabolism of glucose and glutamine, linking them to early changes in glutamate-derived metabolites under diabetic and non-diabetic conditions, and to sex-specific mechanisms of transcriptional and metabolic regulation. Our findings pave the way to new avenues of research based on patient sex, with the potential to improve monitoring and prevention of DKD in men and women. 


\section{METHODS}

\section{Cell culture}

PTECs were purchased from Lonza Walkersville Inc (Walkersville, MD). They were cultured in custom-made Dulbecco's modified Eagle's medium (DMEM) containing 5.55mM D-glucose, 4mM L-glutamine, and $1 \mathrm{mM}$ sodium pyruvate. Growth DMEM media was supplemented with $10 \% \mathrm{v} / \mathrm{v}$ dialyzed fetal bovine serum (FBS), 10ng/mL EGF, 1x of Transferrin/Insulin/Selenium (Invitrogen), $0.05 \mathrm{M}$ hydrocortisone, $50 \mathrm{units} / \mathrm{mL}$ penicillin, and $50 \mathrm{~g} / \mathrm{mL}$ streptomycin, as previously described $^{31,154}$. All experiments were performed at passage 5. To study the effects of cell sex, PTECs from 4 different male donors and 5 different female donors were studied. To study the effects of sex hormones in male and female PTECs, cells were serum-starved for $24 \mathrm{~h}$ and treated with 100nM DHT (D-073 Sigma) or 100nM EST (3301 Sigma) for 16h or 24h. Ethanol-treated cells were used as controls (CONT). After stimulation, cell media were collected and centrifuged at $2000 \mathrm{G}$ for $10 \mathrm{~min}$ at $4^{\circ} \mathrm{C}$, and supernatants were stored at $-80^{\circ} \mathrm{C}$. For gene expression experiments, cells were washed with PBS, harvested with trypsin, and snap-frozen at $80^{\circ} \mathrm{C}$ until further analysis. See Supplemental Methods for experimental details on high glucose and pharmacologic inhibition studies.

\section{Assessment of metabolic function in kidney cells}

Glycolysis was assessed in male and female PTECs by measuring the extracellular acidification rate (ECAR) in a Seahorse XFe96 analyzer (Agilent). Oxygen consumption rate (OCR) was also monitored. Upon confluence, cells were detached with $0.25 \%$ trypsin for $5 \mathrm{~min}$ at $37^{\circ} \mathrm{C}$, and subsequently seeded in a Seahorse XFe96 Cell Culture Microplate at a density of 15,000 cells/well in $100 \mu \mathrm{L}$ of DMEM complete media. After letting them adhere for 4-6h, PTECs were starved for $24 \mathrm{~h}$ and exposed to the treatment of interest. One hour prior to the assay, starvation DMEM media was removed, and cells were washed with phenol-free basal media (Agilent) and 
exposed to $150 \mu \mathrm{L}$ of minimal substrate assay media. The assay media was prepared freshly by adding $2 \mathrm{mM}$ glutamine, $1 \mathrm{mM}$ pyruvate, and $5.55 \mathrm{mM}$ glucose (normal glucose experiments) or $25 \mathrm{mM}$ glucose (high glucose experiments) to the basal media. For substrate restriction experiments, the assay media was prepared with only glucose $(5.55 \mathrm{mM})$, glutamine $(2 \mathrm{mM})$, or pyruvate $(1 \mathrm{mM})$. The same concentrations of sex hormones (100nM for normal glucose conditions and $1 \mathrm{nM}$ for high glucose conditions) were maintained during this acclimatization step. See Supplemental Methods for details about induction of metabolic stress and calculation of ECAR- and OCR-related parameters in PTECs.

\section{Glucose uptake}

Glucose uptake in male and female PTECs was measured with a Glucose Uptake-Glo ${ }^{\text {TM }}$ Assay Kit (Promega), following manufacturer instructions (see Supplemental Methods).

\section{Cell injury}

\section{Cellular oxidative stress and apoptosis}

Oxidative stress in male and female PTECs was assessed by measuring the intracellular levels of superoxide ion with the Cellular ROS Assay Kit (Red) (Abcam) following the manufacturer instructions. In turn, early apoptosis was assessed by measuring the levels of phosphatidylserine on the cell surface using the Apoptosis/Necrosis Assay Kit (Abcam) (see Supplemental Methods).

\section{Lactate dehydrogenase release}

Release of LDH in the cell supernatant was assessed as a marker of cell death in male and female PTECs using the CytoTox96® Cytotoxicity Assay (Promega) following the manufacturer instructions (see Supplemental Methods). 


\section{Cell viability}

\section{Cellular ATP levels}

Intracellular levels of ATP in male and female PTECs were measured with a CellTiter-Glo 2.0 Assay Kit (Promega), following manufacturer instructions (see Supplemental Methods).

\section{MTT assay}

Cell viability was also assessed in male and female PTECs using the MTT assay (Sigma), following manufacturer instructions (see Supplemental Methods).

\section{Cell metabolome}

\section{Sample preparation}

Male and female PTECs were grown on 6-well plates and serum starved as described above. The steady-state intracellular metabolome was then determined after $16 \mathrm{~h}$ of exposure to normal glucose starvation conditions, using liquid chromatography-mass spectrometry (see Supplemental Methods).

\section{Animal studies}

To assess sex differences in glucose and glutamine metabolism in vivo, the kidneys and the plasma of 8-week- and 16-week-old C57BL/6 healthy male and female mice were studied. Male and female diabetic Akita (Ins2WT/C96Y) mice of the same age groups were studied in parallel to assess the effect of sex in the context of type 1 diabetes. To evaluate the role of male and female sex hormones in the kidney levels of key transcriptional regulators, 10-week-old C57BL/6 healthy male and female mice were subjected to gonadectomy (GDX) and ovariectomy (OVX), respectively (see Supplemental Methods). 
To assess the effect of androgens on the urine excretion of glucose, lactate, glutamine, and glutamate, we studied the effect of GDX in a different colony of C57BL/6 10-week-old healthy male mice, as previously described ${ }^{34}$ (see Supplemental Methods).

At the end of each follow-up, morning spot urine was collected through abdominal massage, cleared by centrifugation at $8,000 \mathrm{G}$ for $10 \mathrm{~min}$, and stored at $-80^{\circ} \mathrm{C}$ until further analysis. Mice were then anesthetized with isoflurane and sacrificed by terminal surgery. Blood was collected by cardiac puncture in heparinized tubes (Sarstedt). Plasma was subsequently obtained by centrifugation at $8,000 \mathrm{G}$ for $10 \mathrm{~min}$ and stored at $-80^{\circ} \mathrm{C}$. Kidneys were removed, weighted, snap frozen in liquid nitrogen and kept at $-80^{\circ} \mathrm{C}$ until further analysis. Five to eight animals were studied in each experimental group.

Mouse studies were performed at the Division of Comparative Medicine at University of Toronto (healthy controls and diabetic Akita mice), at the Laval University Animal Supply Facility (GDX and OVX male and female mice), and at the Animal Facility of Barcelona Biomedical Research Park (female, male, and GDX male mice). Mice were housed in ventilated cages with full access to chow and water, in a controlled-temperature environment maintained under a $12 \mathrm{~h}$ light/dark cycle. All experiments were conducted under the guidelines of the University of Toronto Animal Care Committee, the Laval University Animal Care Committee, and the Ethical Committee of Animal Experimentation of Barcelona Biomedical Research Park (CEEA-PRBB). All efforts were made to use the minimal number of mice and minimize animal suffering.

\section{Targeted metabolite measurements}

Levels of glucose, lactate, glutamine, and glutamate were measured in cell supernatants, plasma and urine using a BioProfile ${ }^{\circledR}$ FLEX2 ${ }^{\mathrm{TM}}$ Automated Cell Culture Analyzer (Nova Biomedical) (see Supplemental Methods). 


\section{Gene expression}

Gene expression studies were conducted on RNA extracted from PTEC cell pellets or from 30$50 \mathrm{mg}$ of mouse kidney cortex using the RNAeasy Mini Kit (Qiagen) (see Supplemental Methods). After quantifying RNA concentration in a Nanodrop instrument (Thermo) 300-700ng of RNA were retrotranscribed to cDNA using the High-Capacity cDNA Reverse Transcription Kit (Applied Biosystems). For the in vitro experiments, male and female PTECs were treated with vehicle, 100nM DHT, or 100nM EST for 16-24h under normoglycemic conditions. In these cells, gene levels of AR, KDM6A, ZFX, DDX3X, HNF4A, ZFY, EIF1AY, RPS4Y1, UTY, DDX3Y, and KDM5D were measured by real-time quantitative PCR using a Power SYBR® Green PCR Master Mix reagent (Applied Biosystems) and normalized to HPRT1 or RPL31. The fluorescent signal was measured in a StepOnePlus System (Applied Biosystems) for 96-well plates, and in a LightCycler® 480 Instrument II (Roche) for 384-well plates. For the in vivo experiments, gene levels of Slc38a3, Gls, Glud1, Oxgr1, Kdm6a, Hnf4a, Zfy, Eif1ay, Uty, and Ddx3y were measured and normalized to Hprt1 or Rp/31. All primer sequences employed in this study are summarized in Table S7.

\section{Human studies}

\section{Clinical cohort}

We examined sex differences in the blood metabolome of individuals from the iCARE cohort (University of Manitoba). iCARE is a prospective, observational cohort study to identify risk factors for early onset albuminuria and progression of CKD in male and female adolescents with T2DM $(n=322)^{155}$. These patients were studied together with controls without diabetes of the same age group and ethnicity $(n=139)$. Metabolome analysis was performed on serum and/or urine samples collected from a subgroup of 204 participants of the iCARE cohort (15 males without T2DM, 30 females without T2DM, 52 males with T2DM, and 107 females with T2DM). Specifically, 
metabolite measurements were performed on serum samples from 155/204 cases (14 males and 28 females without diabetes, 36 males and 77 females with T2DM) and on timed overnight/first morning urine samples from 180/204 cases (15 males and 27 females without diabetes, 47 males and 91 females with T2DM) (Fig.S9).

\section{Serum and urine metabolome analysis}

The 155 serum and 180 urine samples of the iCARE cohort were prepared using the automated MicroLab STAR® system (Hamilton Company). The metabolome was measured in the serum and urine sample aliquots following an untargeted UPLC-MS/MS approach (Metabolon Inc, Durham, North Carolina). Raw data files from the MS runs were extracted, peak-identified, and processed for QC using Metabolon's hardware and software. Curation and QC processes were conducted by Metabolon data analysts to ensure accurate and consistent identification of true chemical entities. After curation, peak quantification for each metabolite was preformed using the area-under-the-curve (see Supplemental Methods).

\section{Bioinformatics}

Determination of sex-biased kidney genes in the human kidney tubulointerstitium

To identify genes differentially expressed between male and female kidneys that could relate to our findings in tubular cells we analyzed publicly available gene expression data in Nephroseq database. The analysis led to the identification of 196 genes significantly upregulated in the male tubulointerstitium and 139 genes significantly upregulated in the female tubulointerstitium (Table S5, Fig.S7) (see Supplemental Methods). These two lists of genes were used to identify transcriptional regulators linked to male or female sex, as described below. 


\section{Prediction of sex-specific transcriptional regulators of human kidney signatures}

To identify transcriptional mechanisms linked to the effect of sex hormones in the kidney tubule, we studied computationally which regulators are predicted to target the 60 proteins upregulated by DHT, as well as the 18 upregulated by EST, in PTECs ${ }^{31}$. To account for sex chromosome effects on the kidney tubule, we studied which regulators are predicted to target the 196 genes significantly upregulated in the male tubulointerstitium and the 139 genes significantly upregulated in the female tubulointerstitium (Table S5, Fig.S7). We queried these 4 lists of proteins and genes in CATRIN 1.0, a transcriptional regulator database that integrates the findings of 15 stand-alone transcriptional regulator databases (http://ophid.utoronto.ca/Catrin). Regulators of the genes of interest were identified using CATRIN interaction (excluding TF2DNA.experimental) and hypergeometric test was performed to identify which molecules significantly regulated the lists of genes of interest (see Supplemental methods).

\section{Statistical analysis}

The statistical analysis of our in vitro and in vivo data was performed using GraphPad Prism v9.1.2. Normalized serum and urine metabolome data was analyzed using $R^{156}$ version 4.0.2. Data from 65 metabolites of interest in serum and 19 metabolites in urine was analyzed using three statistical methods (see Supplemental Methods). 


\section{AUTHOR CONTRIBUTIONS}

SC-F and AK conceived the study.

SC-F, OZ, HR, and AK participated in study design.

SC-F and OZ carried out the in vitro experiments.

SC-F, MR, MJS, AI, JL-P, SP, KC, DS, and JWS retrieved and provided the biospecimens for the in vivo studies.

ABD, BW, JMM, and TDB provided clinical and metabolome data from the iCARE cohort for the human studies and helped with the analyses of the clinical data and the conceptual advance.

SC-F, OZ, CP, MK, CMM, HR, and AK analyzed the data.

SF, AS, AB, and MC provided technical support.

SC-F, OZ, CP, MK, IJ, MW, ABD, BW, JMM, TDB, and AK drafted, revised, and edited the paper; and all authors approved the final version of the manuscript. 


\section{ACKNOWLEDGEMENTS}

We thank Madhurangi Arambewela, Shilpa Balaji, and Stefan Petrovic for their technical support, and Brenden Dufault for conducting statistical analyses. We would like to thank the patients and their families for participating in the iCARE research study and for generously donating the biospecimens.

\section{FUNDING}

AK is supported by the Canadian Institutes of Health Research (CIHR) Catalyst grant 347479, Canada Foundation for Innovation (CFI) grant 37205, and Kidney Research Scientist Core Education and National Training (KRESCENT) program grants CIHR148204, KRES160004, and KRES160005. SC-F is supported by the KRESCENT program (2019KPPDF637713). IJ and computational analyses were in part supported by the Schroeder Arthritis Institute at the University Health Network, funding from Natural Sciences Research Council (NSERC \#203475), Canada Foundation for Innovation (CFI \#225404, \#30865), Ontario Research Fund (RDI \#34876), IBM and lan Lawson van Toch Fund. JWS is supported by the CIHR Strategy for Patient-Oriented Research (SPOR) Program and CanSOLVE CKD. MSJ was supported by a grant from the FONDO DE INVESTIGACIÓN SANITARIA-FEDER, ISCIII (\#PI17/00257) and REDINREN (\#RD16/0009/0030). DS is supported by the Canadian Institutes of Health Research (CIHR) 119836 and a Canada Foundation for Innovation (CFI) grant (\#10472). DS and HC are Fonds de recherche du Québec (Santé) Junior 2 scholars. The iCARE study was funded by a CIHR operating grant and received funding from the CIHR SPOR Program and CanSOLVE CKD. 


\section{REFERENCES}

1. Saran R, Robinson B, Abbott KC, et al. US Renal Data System 2016 Annual Data Report: Epidemiology of Kidney Disease in the United States. American Journal of Kidney Diseases. 2017;69(3):A7-A8. doi:10.1053/j.ajkd.2016.12.004

2. de Boer IH, Rue TC, Hall YN, Heagerty PJ, Weiss NS, Himmelfarb J. Temporal trends in the prevalence of diabetic kidney disease in the United States. JAMA. 2011;305(24):2532-2539. doi:10.1001/jama.2011.861

3. A M , M S, I W, et al. Cumulative risk, age at onset, and sex-specific differences for developing end-stage renal disease in young patients with type 1 diabetes: a nationwide population-based cohort study. Diabetes. 2010;59(7):1803-1808. doi:10.2337/DB09-1744

4. MA G, P H, K B-J, HH P. Risk factors for development of incipient and overt diabetic nephropathy in patients with non-insulin dependent diabetes mellitus: prospective, observational study. $B M J$ (Clinical research ed). 1997;314(7083):783-788. doi:10.1136/BMJ.314.7083.783

5. $\quad F P, I M, K L T$, et al. Sex-related differences in diabetic kidney disease: A review on the mechanisms and potential therapeutic implications. Journal of diabetes and its complications. 2021;35(4). doi:10.1016/J.JDIACOMP.2020.107841

6. SD S, W T, I de B, JD B, MW S. Gender and elevated albumin excretion in the Diabetes Control and Complications Trial/Epidemiology of Diabetes Interventions and Complications (DCCT/EDIC) cohort: role of central obesity. American journal of kidney diseases : the official journal of the National Kidney Foundation. 2006;47(2):223-232. doi:10.1053/J.AJKD.2005.10.021

7. Raile K, Galler A, Hofer S, et al. Diabetic nephropathy in 27,805 children, adolescents, and adults with type 1 diabetes: Effect of diabetes duration, A1C, hypertension, dyslipidemia, diabetes onset, and sex. Diabetes Care. 2007;30(10):2523-2528. doi:10.2337/dc07-0282

8. $R M, G D, \cup D M$, et al. Arterial hypertension and microalbuminuria in IDDM: the Italian Microalbuminuria Study. Diabetologia. 1994;37(10):1015-1024. doi:10.1007/BF00400465

9. R R, CA C, KI T, AI A, RR H. Risk factors for renal dysfunction in type 2 diabetes: U.K. Prospective Diabetes Study 74. Diabetes. 2006;55(6):1832-1839. doi:10.2337/DB05-1620

10. $P H, L T, P R$, et al. Predictors for the development of microalbuminuria and macroalbuminuria in patients with type 1 diabetes: inception cohort study. BMJ (Clinical research ed). 2004;328(7448):1105-1108. doi:10.1136/BMJ.38070.450891.FE

11. Giandalia A, Edoardo Giuffrida A, Gembillo G, et al. Molecular Sciences Gender Differences in Diabetic Kidney Disease: Focus on Hormonal, Genetic and Clinical Factors. Published online 2021. doi:10.3390/ijms22115808

12. Bhargava P, Schnellmann RG. Mitochondrial energetics in the kidney. Nature reviews Nephrology. 2017;13(10):629. doi:10.1038/NRNEPH.2017.107 
13. Bonora M, Patergnani S, Rimessi A, et al. ATP synthesis and storage. Purinergic Signalling. 2012;8(3):343-357. doi:10.1007/s11302-012-9305-8

14. Yoo HC, Yu YC, Sung Y, Han JM. Glutamine reliance in cell metabolism. Experimental and Molecular Medicine. 2020;52(9):1496-1516. doi:10.1038/s12276-020-00504-8

15. Brownlee M. The Pathobiology of Diabetic Complications A Unifying Mechanism.; 2004. Accessed July 30, 2019. https://diabetes.diabetesjournals.org/content/diabetes/54/6/1615.full.pdf

16. Koppenol WH, Bounds PL, Dang C v. Otto Warburg's contributions to current concepts of cancer metabolism. Nature Reviews Cancer. 2011;11(5):325-337. doi:10.1038/nrc3038

17. Sas KM, Kayampilly $P$, Byun J, et al. Tissue-specific metabolic reprogramming drives nutrient flux in diabetic complications. JCl insight. 2016;1(15):e86976. doi:10.1172/jci.insight.86976

18. Zhang G, Darshi M, Sharma K. The Warburg Effect in Diabetic Kidney Disease. Seminars in Nephrology. 2018;38(2):111-120. doi:10.1016/j.semnephrol.2018.01.002

19. Kanwar YS, Sun L, Xie P, Liu FY, Chen S. A glimpse of various pathogenetic mechanisms of diabetic nephropathy. Annual Review of Pathology: Mechanisms of Disease. 2011;6:395-423. doi:10.1146/annurev.pathol.4.110807.092150

20. Reidy K, Kang HM, Hostetter T, Susztak K. Molecular mechanisms of diabetic kidney disease. The Journal of clinical investigation. 2014;124(6):2333-2340. doi:10.1172/JCI72271

21. HM K, SH A, P C, et al. Defective fatty acid oxidation in renal tubular epithelial cells has a key role in kidney fibrosis development. Nature medicine. 2015;21(1):37-46. doi:10.1038/NM.3762

22. Zheng W, Guo J, Liu Z-S. Effects of metabolic memory on inflammation and fibrosis associated with diabetic kidney disease: an epigenetic perspective. Clinical Epigenetics 2021 13:1. 2021;13(1):1-16. doi:10.1186/S13148-021-01079-5

23. Bansal A, Balasubramanian S, Dhawan S, Leung A, Chen Z, Natarajan R. Integrative Omics Analyses Reveal Epigenetic Memory in Diabetic Renal Cells Regulating Genes Associated With Kidney Dysfunction. Diabetes. 2020;69(11):2490-2502. doi:10.2337/DB20-0382

24. Hodgkins KS, Schnaper HW. Tubulointerstitial injury and the progression of chronic kidney disease. Pediatric nephrology (Berlin, Germany). 2012;27(6):901-909. doi:10.1007/s00467-0111992-9

25. Zoja C, Abbate M, Remuzzi G. Progression of renal injury toward interstitial inflammation and glomerular sclerosis is dependent on abnormal protein filtration. Nephrology Dialysis Transplantation. 2015;30(5):706-712. doi:10.1093/ndt/gfu261

26. C W, SE I, JM L, et al. Empagliflozin and Progression of Kidney Disease in Type 2 Diabetes. The New England journal of medicine. 2016;375(4):323-334. doi:10.1056/NEJMOA1515920

27. V P, MJ J, B N, et al. Canagliflozin and Renal Outcomes in Type 2 Diabetes and Nephropathy. The New England journal of medicine. 2019;380(24):2295-2306. doi:10.1056/NEJMOA1811744 
28. DC W, BV S, N J, et al. Effects of dapagliflozin on major adverse kidney and cardiovascular events in patients with diabetic and non-diabetic chronic kidney disease: a prespecified analysis from the DAPA-CKD trial. The lancet Diabetes \& endocrinology. 2021;9(1):22-31. doi:10.1016/S22138587(20)30369-7

29. DL B, M S, B P, et al. Sotagliflozin in Patients with Diabetes and Chronic Kidney Disease. The New England journal of medicine. 2021;384(2):129-139. doi:10.1056/NEJMOA2030186

30. HJL H, BV S, R C-R, et al. Dapagliflozin in Patients with Chronic Kidney Disease. The New England journal of medicine. 2020;383(15):1436-1446. doi:10.1056/NEJMOA2024816

31. Clotet S, Soler MJ, Riera M, et al. SILAC-Based Proteomics of Primary Human Kidney Cells Reveals a Novel Link between Male Sex Hormones and Impaired Energy Metabolism in Diabetic Kidney Disease. Molecular \& Cellular Proteomics. Published online January 4, 2017:mcp.M116.061903. doi:10.1074/mcp.M116.061903

32. Clotet-Freixas S, Soler MJ, Palau V, et al. Sex dimorphism in ANGII-mediated crosstalk between ACE2 and ACE in diabetic nephropathy. Laboratory investigation; a journal of technical methods and pathology. 2018;98(9):1237-1249. doi:10.1038/s41374-018-0084-x

33. Clotet S, Riera M, Pascual J, Soler MJ. RAS and sex differences in diabetic nephropathy. American Journal of Physiology - Renal Physiology. Published online March 9, 2016:ajprenal.00292.2015. doi:10.1152/ajprenal.00292.2015

34. Clotet S, Soler MJ, Rebull M, et al. Gonadectomy prevents the increase in blood pressure and glomerular injury in angiotensin-converting enzyme 2 knockout diabetic male mice. Effects on renin-angiotensin system. Journal of hypertension. Published online July 1, 2016. doi:10.1097/HJH.0000000000001015

35. Van JAD, Clotet-Freixas S, Zhou J, et al. Peptidomic analysis of urine from youths with early type 1 diabetes reveals novel bioactivity of uromodulin peptides in vitro. Molecular and Cellular Proteomics. 2020;19(3):501-517. doi:10.1074/mcp.RA119.001858

36. Van JAD, Scholey JW, Konvalinka A. Insights into Diabetic Kidney Disease Using Urinary Proteomics and Bioinformatics. Journal of the American Society of Nephrology. 2017;28(4):10501061. doi:10.1681/ASN.2016091018

37. Xu Q, Wells CC, Garman JH, Asico L, Escano CS, Maric C. Imbalance in sex hormone levels exacerbates diabetic renal disease. Hypertension. 2008;51(4 PART 2 SUPPL.):1218-1224. doi:10.1161/HYPERTENSIONAHA.107.100594

38. Shepard BD. Sex differences in diabetes and kidney disease: Mechanisms and consequences. American Journal of Physiology - Renal Physiology. 2019;317(2):F456-F462. doi:10.1152/ajprenal.00249.2019

39. Maric-Bilkan C. Sex Differences in Diabetic Kidney Disease. Mayo Clinic Proceedings. 2020;95(3):587-599. doi:10.1016/j.mayocp.2019.08.026 
40. Verzola D, Villaggio B, Procopio V, et al. Androgen-mediated apoptosis of kidney tubule cells: role of c-Jun amino terminal kinase. Biochemical and biophysical research communications.

2009;387(3):531-536. doi:10.1016/j.bbrc.2009.07.056

41. Verzola D, Gandolfo MT, Salvatore F, et al. Testosterone promotes apoptotic damage in human renal tubular cells. Kidney Int. 2004;65(4):1252-1261. doi:10.1111/j.1523-1755.2004.00497.x [doi]

42. Negulescu O, Bognar I, Lei J, Devarajan P, Silbiger S, Neugarten J. Estradiol reverses TGF-beta1induced mesangial cell apoptosis by a casein kinase 2-dependent mechanism. Kidney international. 2002;62(6):1989-1998. doi:10.1046/j.1523-1755.2002.00679.x

43. Clayton JA, Collins FS. Policy: NIH to balance sex in cell and animal studies. Nature. 2014;509(7500):282-283. Accessed June 18, 2016. http://www.ncbi.nlm.nih.gov/pubmed/24834516

44. Arnold AP, Cassis LA, Eghbali M, Reue K, Sandberg K. Sex hormones and sex chromosomes cause sex differences in the development of cardiovascular diseases. Arteriosclerosis, Thrombosis, and Vascular Biology. 2017;37(5):746-756. doi:10.1161/ATVBAHA.116.307301

45. Pihlajamaa P, Sahu B, Lyly L, Aittomäki V, Hautaniemi S, Jänne OA. Tissue-specific pioneer factors associate with androgen receptor cistromes and transcription programs. EMBO Journal. 2014;33(4):312-326. doi:10.1002/embj.201385895

46. Jae Han $\mathrm{H}$, Jung Lee $\mathrm{Y}$, Hyung Park $\mathrm{S}$, Hern Lee J, Taub M, Jae H. High glucose-induced oxidative stress inhibits $\mathrm{Na} / g$ lucose cotransporter activity in renal proximal tubule cells. Am J Physiol Renal Physiol. 2005;288:988-996. doi:10.1152/ajprenal.00327.2004.-Oxidative

47. Giacco F, Brownlee M. Oxidative stress and diabetic complications. Circulation Research. 2010;107(9):1058-1070. doi:10.1161/CIRCRESAHA.110.223545

48. Flutamide - an overview / ScienceDirect Topics. Accessed June 21, 2021. https://www.sciencedirect.com/topics/chemistry/flutamide

49. Ito Y, Sadar MD. Enzalutamide and blocking androgen receptor in advanced prostate cancer: Lessons learnt from the history of drug development of antiandrogens. Research and Reports in Urology. 2018;10:23-32. doi:10.2147/RRU.S157116

50. Srivastava S. Emerging insights into the metabolic alterations in aging using metabolomics. Metabolites. 2019;9(12). doi:10.3390/metabo9120301

51. Yang $C$, Ko B, Hensley CT, et al. Glutamine oxidation maintains the TCA cycle and cell survival during impaired mitochondrial pyruvate transport. Molecular Cell. 2014;56(3):414-424. doi:10.1016/j.molcel.2014.09.025

52. Altman BJ, Stine ZE, Dang C v. From Krebs to clinic: Glutamine metabolism to cancer therapy. Nature Reviews Cancer. 2016;16(10):619-634. doi:10.1038/nrc.2016.71 
53. Fang $\mathrm{F}, \mathrm{Bae} \mathrm{EH}, \mathrm{Hu} \mathrm{A}$, et al. Deletion of the gene for adiponectin accelerates diabetic nephropathy in the Ins2 +/C96Y mouse. Diabetologia. 2015;58(7):1668-1678.

doi:10.1007/s00125-015-3605-9

54. Fang $\mathrm{F}, \mathrm{Bae} \mathrm{E}-\mathrm{H}, \mathrm{Hu} \mathrm{A}$, et al. Deletion of the gene for adiponectin accelerates diabetic nephropathy in the Ins2 (+/C96Y) mouse. Diabetologia. 2015;58(7):1668-1678.

doi:10.1007/s00125-015-3605-9

55. AB D, BA W, EA S, et al. The Improving Renal Complications in Adolescents With Type 2 Diabetes Through the REsearch (iCARE) Cohort Study: rationale and Protocol. Canadian journal of diabetes. 2014;38(5):349-355. doi:10.1016/J.JCJD.2014.07.224

56. Ribas V, García-Ruiz C, Fernández-Checa JC. Glutathione and mitochondria. Frontiers in Pharmacology. 2014;5 JUL:151. doi:10.3389/fphar.2014.00151

57. Owen JB, Allan Butterfiel D. Measurement of oxidized/reduced glutathione ratio. In: Methods in Molecular Biology. Vol 648. Humana Press Inc.; 2010:269-277. doi:10.1007/978-1-60761-7563_18

58. Bachhawat AK, Yadav S. The glutathione cycle: Glutathione metabolism beyond the $\gamma$-glutamyl cycle. IUBMB Life. 2018;70(7):585-592. doi:10.1002/iub.1756

59. Boysen G. The Glutathione Conundrum: Stoichiometric Disconnect between Its Formation and Oxidative Stress. Chemical Research in Toxicology. 2017;30(5):1113-1116.

doi:10.1021/acs.chemrestox.7b00018

60. Hanigan MH. Gamma-glutamyl transpeptidase: Redox regulation and drug resistance. In: Advances in Cancer Research. Vol 122. Academic Press Inc.; 2014:103-141. doi:10.1016/B978-012-420117-0.00003-7

61. Sonnewald U. Glutamate synthesis has to be matched by its degradation - Where do all the carbons go? Journal of Neurochemistry. 2014;131(4):399-406. doi:10.1111/jnc.12812

62. Woroniecka KI, Park ASD, Mohtat D, Thomas DB, Pullman JM, Susztak K. Transcriptome analysis of human diabetic kidney disease. Diabetes. 2011;60(9):2354-2369. doi:10.2337/db10-1181

63. Ju W, Nair V, Smith S, et al. Tissue transcriptome-driven identification of epidermal growth factor as a chronic kidney disease biomarker. Science Translational Medicine. 2015;7(316). doi:10.1126/scitranslmed.aac7071

64. Lindenmeyer MT, Eichinger F, Sen K, et al. Systematic analysis of a novel human renal glomerulus-enriched gene expression dataset. PLOS ONE. 2010;5(7). doi:10.1371/journal.pone.0011545

65. Tukiainen T, Villani AC, Yen A, et al. Landscape of $X$ chromosome inactivation across human tissues. Nature. 2017;550(7675):244-248. doi:10.1038/nature24265

66. Hyun K, Jeon J, Park K, Kim J. Writing, erasing and reading histone lysine methylations. Experimental and Molecular Medicine. 2017;49(4). doi:10.1038/emm.2017.11 
67. Chang S, Yim S, Park H. The cancer driver genes IDH1/2, JARID1C/ KDM5C, and UTX/ KDM6A: crosstalk between histone demethylation and hypoxic reprogramming in cancer metabolism. Experimental and Molecular Medicine. 2019;51(6). doi:10.1038/s12276-019-0230-6

68. Li X, Zhang $Q$, Shi $Q$, et al. Demethylase Kdm6a epigenetically promotes IL-6 and IFN- $\beta$ production in macrophages. Journal of Autoimmunity. 2017;80:85-94. doi:10.1016/j.jaut.2017.02.007

69. Huot $\mathrm{G}$, Vernier M, Bourdeau V, et al. CHES1/FOXN3 regulates cell proliferation by repressing PIM2 and protein biosynthesis. Molecular Biology of the Cell. 2014;25(5):554-565.

doi:10.1091/mbc.E13-02-0110

70. Cribbs AP, Terlecki-Zaniewicz S, Philpott M, et al. Histone H3K27me3 demethylases regulate human Th17 cell development and effector functions by impacting on metabolism. Proceedings of the National Academy of Sciences of the United States of America. 2020;117(11):6056-6066. doi:10.1073/pnas.1919893117

71. Doñas C, Neira J, Osorio-Barrios F, et al. The demethylase inhibitor GSK-J4 limits inflammatory colitis by promoting de novo synthesis of retinoic acid in dendritic cells. Scientific Reports. 2021;11(1):1342. doi:10.1038/s41598-020-79122-3

72. Heiden MGV, Cantley LC, Thompson CB. Understanding the warburg effect: The metabolic requirements of cell proliferation. Science. 2009;324(5930):1029-1033. doi:10.1126/science.1160809

73. Maestro M, Cardalda C, Boj S, Luco R, Servitja J, Ferrer J. Distinct roles of HNF1 $\beta$, HNF1 $\alpha$, and $\mathrm{HNF} 4 \alpha$ in regulating pancreas development, $\beta$-cell function and growth. In: Endocrine Development. Vol 12. ; 2007:33-45. doi:10.1159/000109603

74. Cattin A-L, le Beyec J, Barreau F, et al. Hepatocyte Nuclear Factor $4 \alpha$, a Key Factor for Homeostasis, Cell Architecture, and Barrier Function of the Adult Intestinal Epithelium. Molecular and Cellular Biology. 2009;29(23):6294-6308. doi:10.1128/mcb.00939-09

75. Fang B, Mane-Padros D, Bolotin E, Jiang T, Sladek FM. Identification of a binding motif specific to HNF4 by comparative analysis of multiple nuclear receptors. Nucleic Acids Research. 2012;40(12):5343-5356. doi:10.1093/nar/gks190

76. Velázquez-Villegas LA, Charabati T, Contreras A v., Alemán G, Torres N, Tovar AR. PPAR $\alpha$ downregulates hepatic glutaminase expression in mice fed diets with different protein: Carbohydrate ratios. Journal of Nutrition. 2016;146(9):1634-1640. doi:10.3945/jn.116.232868

77. Stanulović VS, Kyrmizi I, Kruithof-De Julio M, et al. Hepatic HNF4 $\alpha$ deficiency induces periportal expression of glutamine synthetase and other pericentral enzymes. Hepatology. 2007;45(2):433444. doi:10.1002/hep.21456

78. Dubois V, Staels B, Lefebvre P, Verzi MP, Eeckhoute J. Control of Cell Identity by the Nuclear Receptor HNF4 in Organ Pathophysiology. Cells. 2020;9(10). doi:10.3390/cells9102185

79. Martovetsky G, Tee JB, Nigam SK. Hepatocyte nuclear factors $4 \alpha$ and $1 \alpha$ regulate kidney developmental expression of drug-metabolizing enzymes and drug transporters. Molecular Pharmacology. 2013;84(6):808-823. doi:10.1124/mol.113.088229 
80. P D, J P, C HDP, et al. The Nuclear Receptor ESRRA Protects from Kidney Disease by Coupling Metabolism and Differentiation. Cell metabolism. 2021;33(2):379-394.e8.

doi:10.1016/J.CMET.2020.11.011

81. Kiselyuk A, Lee SH, Farber-Katz S, et al. HNF4 $\alpha$ antagonists discovered by a high-throughput screen for modulators of the human insulin promoter. Chemistry and Biology. 2012;19(7):806818. doi:10.1016/j.chembiol.2012.05.014

82. Sex-related differences in diabetic kidney disease: A review on the mechanisms and potential therapeutic implications | Elsevier Enhanced Reader. Accessed July 6, 2021. https://reader.elsevier.com/reader/sd/pii/S1056872720306425?token=5A33E126033DB9E51EE7 0823D0865F60C3B646106DEB409EBE4C64CAB215FA6986F2F9BA081A3369A15F0681E28E55E5 \&originRegion=us-east-1\&originCreation $=20210706191327$

83. NJ R, JM S, M C, RJ M, G J, El E. Clinical predictive factors in diabetic kidney disease progression. Journal of diabetes investigation. 2017;8(1):6-18. doi:10.1111/JDI.12533

84. CM-B. Sex differences in micro- and macro-vascular complications of diabetes mellitus. Clinical science (London, England : 1979). 2017;131(9):833-846. doi:10.1042/CS20160998

85. Neugarten J, Golestaneh L. Gender and the prevalence and progression of renal disease. Advances in Chronic Kidney Disease. 2013;20(5):390-395. doi:10.1053/j.ackd.2013.05.004

86. Mauvais-Jarvis F. Sex differences in metabolic homeostasis, diabetes, and obesity. Biology of Sex Differences. Published online 2015. doi:10.1186/s13293-015-0033-y

87. Ainsworth C. Genetics: Sex and the single cell. Nature. 2017;550(7674):S6-S8. doi:10.1038/550S6a

88. Skaletsky H, Kuroda-Kawaguchl T, Minx PJ, et al. The male-specific region of the human $\mathrm{Y}$ chromosome is a mosaic of discrete sequence classes. Nature. 2003;423(6942):825-837. doi:10.1038/nature01722

89. Khramtsova EA, Davis LK, Stranger BE. The role of sex in the genomics of human complex traits. Nature Reviews Genetics 2018 20:3. 2018;20(3):173-190. doi:10.1038/s41576-018-0083-1

90. HS, RS B, P K, et al. Human and murine kidneys show gender- and species-specific gene expression differences in response to injury. PloS one. 2009;4(3).

doi:10.1371/JOURNAL.PONE.0004802

91. Bhargava A, Arnold AP, Bangasser DA, et al. Considering Sex as a Biological Variable in Basic and Clinical Studies: An Endocrine Society Scientific Statement. Endocrine Reviews. 2021;XX:1-40. doi:10.1210/endrev/bnaa034

92. Chen X, McClusky R, Itoh Y, Reue K, Arnold AP. X and Y chromosome complement influence adiposity and metabolism in mice. Endocrinology. Published online 2013. doi:10.1210/en.20122098 
93. Shi H, Brown LM, Rahimian R. Sex/Gender Differences in Metabolism and Behavior: Influence of Sex Chromosomes and Hormones. International Journal of Endocrinology. 2015;2015. doi:10.1155/2015/245949

94. Link JC, Chen X, Arnold AP, Reue K. Metabolic impact of sex chromosomes. Adipocyte. 2013;2(2):74. doi:10.4161/ADIP.23320

95. Sandberg K, Ji H. Sex and the renin angiotensin system: Implications for gender differences in the progression of kidney disease. Advances in Renal Replacement Therapy. 2003;10(1):15-23. doi:10.1053/jarr.2003.50006

96. K S. Mechanisms underlying sex differences in progressive renal disease. Gender medicine. 2008;5(1):10-23. doi:10.1016/S1550-8579(08)80004-6

97. Brinson KN. Sex Differences in Renal Inner Medullary Nitric Oxide Synthase Regulation and Nitric Oxide Synthase Contribution to Blood Pressure Control in Hypertension. Published online 2013. Accessed July 8, 2021. https://augusta.openrepository.com/handle/10675.2/344307

98. Ojeda NB, Hennington BS, Williamson DT, et al. OXIDATIVE STRESS CONTRIBUTES TO SEX DIFFERENCES IN BLOOD PRESSURE IN ADULT GROWTH RESTRICTED OFFSPRING. Hypertension. 2012;60(1):114. doi:10.1161/HYPERTENSIONAHA.112.192955

99. RF S, R S, IA Y, K S, DV I. Sex differences in renal mitochondrial function: a hormone-gous opportunity for research. American journal of physiology Renal physiology. 2020;319(6):F1117F1124. doi:10.1152/AJPRENAL.00320.2020

100. B C, MP A, A V, J O, P R, FJ G-P. Skeletal muscle of female rats exhibit higher mitochondrial mass and oxidative-phosphorylative capacities compared to males. Cellular physiology and biochemistry : international journal of experimental cellular physiology, biochemistry, and pharmacology. 2007;19(1-4):205-212. doi:10.1159/000099208

101. AR K, EA A-R, AM M, et al. Sex-specific differences in mitochondria biogenesis, morphology, respiratory function, and ROS homeostasis in young mouse heart and brain. Physiological reports. 2017;5(6). doi:10.14814/PHY2.13125

102. $\mathrm{Z} L, S \mathrm{~L}, \mathrm{X}$ L. The role of metabolic reprogramming in tubular epithelial cells during the progression of acute kidney injury. Cellular and molecular life sciences : CMLS. Published online June 29, 2021. doi:10.1007/S00018-021-03892-W

103. Bhatti JS, Bhatti GK, Reddy PH. Mitochondrial dysfunction and oxidative stress in metabolic disorders - A step towards mitochondria based therapeutic strategies. Biochimica et Biophysica Acta (BBA) - Molecular Basis of Disease. 2017;1863(5):1066-1077. doi:10.1016/J.BBADIS.2016.11.010

104. Bhargava P, Schnellmann RG. Mitochondrial energetics in the kidney. Nature Reviews Nephrology. 2017;13(10):629-646. doi:10.1038/nrneph.2017.107

105. Mather A, Pollock C. Glucose handling by the kidney. Kidney International. 2011;79:S1-S6. doi:10.1038/KI.2010.509 
106. Stumvoll M, Perriello G, Meyer C, Gerich J. Role of glutamine in human carbohydrate metabolism in kidney and other tissues. Kidney International. 1999;55(3):778-792. doi:10.1046/J.15231755.1999.055003778.X

107. NP C. Role of mitochondrial glutaminase in rat renal glutamine metabolism. The Journal of nutrition. 2001;131(9 Suppl). doi:10.1093/JN/131.9.2491S

108. Kang HM, Ahn SH, Choi P, et al. Defective fatty acid oxidation in renal tubular epithelial cells has a key role in kidney fibrosis development. Nature Medicine. 2014;21(1):37-46.

doi:10.1038/nm.3762

109. Forbes JM, Thorburn DR. Mitochondrial dysfunction in diabetic kidney disease. Nature Reviews Nephrology. 2018;14(5):291-312. doi:10.1038/nrneph.2018.9

110. LS G. Sugar or Fat? Renal Tubular Metabolism Reviewed in Health and Disease. Nutrients. 2021;13(5). doi:10.3390/NU13051580

111. Czajka A, Malik AN. Hyperglycemia induced damage to mitochondrial respiration in renal mesangial and tubular cells: Implications for diabetic nephropathy. Redox Biology. 2016;10:100107. doi:10.1016/j.redox.2016.09.007

112. Park $\mathrm{SH}, \mathrm{Choi} \mathrm{HJ}$, Lee $\mathrm{JH}$, Woo $\mathrm{CH}, \mathrm{Kim} \mathrm{JH}$, Han HJ. High glucose inhibits renal proximal tubule cell proliferation and involves PKC, oxidative stress, and TGF- $\beta 1$. Kidney International. 2001;59(5):1695-1705. doi:10.1046/j.1523-1755.2001.0590051695.x

113. Lund J, Ouwens DM, Wettergreen M, Bakke SS, Thoresen GH, Aas V. Increased Glycolysis and Higher Lactate Production in Hyperglycemic Myotubes. Cells. 2019;8(9). doi:10.3390/CELLS8091101

114. Kang HM, Ahn SH, Choi P, et al. Defective fatty acid oxidation in renal tubular epithelial cells plays a key role in kidney fibrosis development. Nature medicine. 2015;21(1):37. doi:10.1038/NM.3762

115. Martínez-Reyes I, Chandel NS. Mitochondrial TCA cycle metabolites control physiology and disease. Nature Communications 2020 11:1. 2020;11(1):1-11. doi:10.1038/s41467-019-13668-3

116. Krumsiek J, Mittelstrass K, Do KT, et al. Gender-specific pathway differences in the human serum metabolome. Metabolomics. 2015;11(6):1815-1833. doi:10.1007/s11306-015-0829-0

117. X Z, H X, J N, et al. Sex-Specific Metabolic Changes in Peripheral Organs of Diabetic Mice. Journal of proteome research. 2020;19(8):3011-3021. doi:10.1021/ACS.JPROTEOME.0C00049

118. S F, A Y, M S, et al. Sex-associated differences in baseline urinary metabolites of healthy adults. Scientific reports. 2018;8(1). doi:10.1038/S41598-018-29592-3

119. JJ L, S L, RL G, et al. Urine Tricarboxylic Acid Cycle Metabolites Predict Progressive Chronic Kidney Disease in Type 2 Diabetes. The Journal of clinical endocrinology and metabolism. 2018;103(12):4357-4364. doi:10.1210/JC.2018-00947

120. PJ S, M D, KM W, et al. Urine metabolites are associated with glomerular lesions in type 2 diabetes. Metabolomics : Official journal of the Metabolomic Society. 2018;14(6). doi:10.1007/S11306-018-1380-6 
121. Darshi M, Espen B van, Sharma K. Metabolomics in Diabetic Kidney Disease: Unraveling the Biochemistry of A Silent Killer. American journal of nephrology. 2016;44(2):92. doi:10.1159/000447954

122. $\mathrm{M} \mathrm{L}, \mathrm{XW}, \mathrm{J} \mathrm{A}$, et al. GC/TOFMS analysis of metabolites in serum and urine reveals metabolic perturbation of TCA cycle in $\mathrm{db} / \mathrm{db}$ mice involved in diabetic nephropathy. American journal of physiology Renal physiology. 2013;304(11):1317-1324. doi:10.1152/AJPRENAL.00536.2012

123. Mulder S, Hammarstedt A, Nagaraj SB, et al. A metabolomics-based molecular pathway analysis of how the sodium-glucose co-transporter-2 inhibitor dapagliflozin may slow kidney function decline in patients with diabetes. Diabetes, Obesity and Metabolism. 2020;22(7):1157-1166. doi:10.1111/DOM.14018

124. Wang L, Ahn YJ, Asmis R. Sexual dimorphism in glutathione metabolism and glutathionedependent responses. Redox Biology. 2020;31:101410. doi:10.1016/J.REDOX.2019.101410

125. Azarova I, Bushueva O, Konoplya A, Polonikov A. Glutathione S-transferase genes and the risk of type 2 diabetes mellitus: Role of sexual dimorphism, gene-gene and gene-smoking interactions in disease susceptibility. Journal of Diabetes. 2018;10(5):398-407. doi:10.1111/1753-0407.12623

126. Díaz A, López-Grueso R, Gambini J, et al. Sex Differences in Age-Associated Type 2 Diabetes in Rats-Role of Estrogens and Oxidative Stress. Oxidative Medicine and Cellular Longevity. 2019;2019. doi:10.1155/2019/6734836

127. Forman $\mathrm{HJ}$, Zhang $\mathrm{H}$, Rinna A. Glutathione: Overview of its protective roles, measurement, and biosynthesis. Molecular aspects of medicine. 2009;30(1-2):1. doi:10.1016/J.MAM.2008.08.006

128. Lu SC. GLUTATHIONE SYNTHESIS. Biochimica et biophysica acta. 2013;1830(5):3143. doi:10.1016/J.BBAGEN.2012.09.008

129. MC T, M B, K S, et al. Diabetic kidney disease. Nature reviews Disease primers. 2015;1. doi:10.1038/NRDP.2015.18

130. Gurley SB, Mach CL, Stegbauer J, et al. Influence of genetic background on albuminuria and kidney injury in Ins2+/C96Y (Akita) mice. American Journal of Physiology - Renal Physiology. 2010;298(3):F788. doi:10.1152/AJPRENAL.90515.2008

131. ON G, C P, P S, et al. Metabolites associate with kidney function decline and incident chronic kidney disease in the general population. Nephrology, dialysis, transplantation : official publication of the European Dialysis and Transplant Association - European Renal Association. 2013;28(8):2131-2138. doi:10.1093/NDT/GFT217

132. Pandey N, Lanke V, Vinod PK. Network-based metabolic characterization of renal cell carcinoma. Scientific Reports 2020 10:1. 2020;10(1):1-13. doi:10.1038/s41598-020-62853-8

133. Wu I-W, Gao S-S, Chou H-C, et al. Integrative metagenomic and metabolomic analyses reveal severity-specific signatures of gut microbiota in chronic kidney disease. Theranostics. 2020;10(12):5398. doi:10.7150/THNO.41725 
134. Kato $M$, Natarajan R. Epigenetics and epigenomics in diabetic kidney disease and metabolic memory. Nature Reviews Nephrology. 2019;15(6):327-345. doi:10.1038/s41581-019-0135-6

135. Fang H, Disteche CM, Berletch JB. X Inactivation and Escape: Epigenetic and Structural Features. Frontiers in Cell and Developmental Biology. 2019;7. doi:10.3389/fcell.2019.00219

136. Itoh Y, Golden LC, Itoh N, et al. The X-linked histone demethylase Kdm6a in CD4+ T lymphocytes modulates autoimmunity. Journal of Clinical Investigation. 2019;129(9):3852-3863.

doi:10.1172/JCI126250

137. Li J, Chen X, McClusky R, et al. The number of X chromosomes influences protection from cardiac ischaemia/reperfusion injury in mice: One $\mathrm{X}$ is better than two. Cardiovascular Research. 2014;102(3):375-384. doi:10.1093/cvr/cvu064

138. McCommis KS, Finck BN. Mitochondrial pyruvate transport: a historical perspective and future research directions. The Biochemical journal. 2015;466(3):443. doi:10.1042/BJ20141171

139. Qi W, Keenan HA, Li Q, et al. Pyruvate kinase M2 activation may protect against the progression of diabetic glomerular pathology and mitochondrial dysfunction. Nature medicine. Published online April 24, 2017. doi:10.1038/nm.4328

140. Cribbs AP, Terlecki-Zaniewicz S, Philpott M, et al. Histone H3K27me3 demethylases regulate human Th17 cell development and effector functions by impacting on metabolism. Proceedings of the National Academy of Sciences of the United States of America. 2020;117(11):6056-6066. doi:10.1073/pnas.1919893117

141. Martovetsky G, Tee JB, Nigam SK. Hepatocyte nuclear factors $4 \alpha$ and $1 \alpha$ regulate kidney developmental expression of drug-metabolizing enzymes and drug transporters. Molecular Pharmacology. 2013;84(6):808-823. doi:10.1124/mol.113.088229

142. Odom DT, Zizlsperger H, Gordon DB, et al. Control of Pancreas and Liver Gene Expression by HNF Transcription Factors. Science. 2004;303(5662):1378-1381. doi:10.1126/science.1089769

143. Marable SS, Chung E, Adam M, Potter SS, Park JS. Hnf4a deletion in the mouse kidney phenocopies Fanconi renotubular syndrome. JCl insight. 2018;3(14). doi:10.1172/jci.insight.97497

144. Pihlajamaa P, Sahu B, Lyly L, Aittomäki V, Hautaniemi S, Jänne OA. Tissue-specific pioneer factors associate with androgen receptor cistromes and transcription programs. EMBO Journal. 2014;33(4):312-326. doi:10.1002/embj.201385895

145. Marable SS, Chung E, Park J-S. Hnf4a-mediated regulation of proximal tubule progenitors in the mouse kidney. bioRxiv. Published online 2020:2020.02.16.951731.

146. Sasaki S, Hara A, Sakaguchi M, Nangaku M, Inoue Y. Hepatocyte nuclear factor $4 \alpha$ regulates megalin expression in proximal tubular cells. Biochemistry and Biophysics Reports. 2019;17:8792. doi:10.1016/j.bbrep.2018.11.010 
147. Pan J, Silva TC, Gull N, et al. Lineage-specific epigenomic and genomic activation of oncogene HNF4A promotes gastrointestinal adenocarcinomas. Cancer Research. 2020;80(13):2722-2736. doi:10.1158/0008-5472.CAN-20-0390

148. Qu M, Duffy T, Hirota T, Kay SA. Nuclear receptor HNF4A transrepresses CLOCK:BMAL1 and modulates tissue-specific circadian networks. Proceedings of the National Academy of Sciences. 2018;115(52):E12305-E12312. doi:10.1073/PNAS.1816411115

149. Niehof M, Borlak J. HNF4 $\alpha$ and the Ca-channel TRPC1 are novel disease candidate genes in diabetic nephropathy. Diabetes. 2008;57(4):1069-1077. doi:10.2337/db07-1065

150. Wang Y, Chaudhari S, Ren Y, Ma R. Impairment of hepatic nuclear factor-4 $\alpha$ binding to the stim1 promoter contributes to high glucose-induced upregulation of stim1 expression in glomerular mesangial cells. American Journal of Physiology - Renal Physiology. 2015;308(10):F1135-F1145. doi:10.1152/ajprenal.00563.2014

151. Bansal A, Balasubramanian S, Dhawan S, Leung A, Chen Z, Natarajan R. Integrative omics analyses reveal epigenetic memory in diabetic renal cells regulating genes associated with kidney dysfunction. Diabetes. 2020;69(11):2490-2502. doi:10.2337/db20-0382

152. Barry WE, Thummel CS. The Drosophila HNF4 nuclear receptor promotes glucose-stimulated insulin secretion and mitochondrial function in adults. eLife. 2016;5(MAY2016). doi:10.7554/eLife.11183

153. Miura A, Yamagata K, Kakei M, et al. Hepatocyte nuclear factor-4 $\alpha$ is essential for glucosestimulated insulin secretion by pancreatic $\beta$-cells. Journal of Biological Chemistry. 2006;281(8):5246-5257. doi:10.1074/jbc.M507496200

154. Konvalinka A, Zhou J, Dimitromanolakis A, et al. Determination of an angiotensin II-regulated proteome in primary human kidney cells by stable isotope labeling of amino acids in cell culture (SILAC). The Journal of biological chemistry. 2013;288(34):24834-24847. doi:10.1074/jbc.M113.485326

155. Dart AB, Wicklow BA, Sellers EA, et al. The Improving Renal Complications in Adolescents With Type 2 Diabetes Through the REsearch (iCARE) Cohort Study: Rationale and Protocol. Canadian Journal of Diabetes. 2014;38(5):349-355. doi:10.1016/j.jcjd.2014.07.224

156. R Foundation for Statistical Computing. R: A language and environment for statistical computing. Vienna, Austria. Published 2014. http://www.gnu.org/copyleft/gpl.html.\%0Ahttp://www.gbif.org/resource/81287 
Table 1. Demographics of adolescents with type 2 diabetes and matched controls. Groupto-group differences in age at baseline, duration of diabetes, HbA1c, BMI, ACR, and eGFR were calculated using the Mann-Whitney test, and data are expressed as median (interquartile range). The eGFR was calculated using the iCARE equation (Dart, et al., Pediatr Nephrol. 2019): $50.7 \times$ $\mathrm{BSA}^{0.816} \times(\text { height }(\mathrm{cm}) / \text { creatinine })^{0.405} \times 0.8994$ if sex $=$ female $\mid 1$ otherwise. Group-to-group differences in the frequency of participants with hypertension were calculated using the Fisher exact test. Abbreviations: T2DM, type 2 diabetes mellitus; HbA1c, glycated hemoglobin; BMI, body mass index; ACR, albumin to creatinine ratio; eGFR, estimated glomerular filtration rate; $\mathrm{N} / \mathrm{A}$, not available.

\begin{tabular}{|c|c|c|c|}
\hline & Controls without T2DM & Patients with T2DM & $P$ value \\
\hline $\mathbf{N}$ & 45 & 159 & $\mathrm{~N} / \mathrm{A}$ \\
\hline Sex (\% Female) & 66.67 & 67.30 & 1.000 \\
\hline Age at Baseline (years) & & & \\
\hline Total & $16.87(13.77,18.10)$ & $14.95(13.14,16.65)$ & 0.020 \\
\hline Male & $14.99(13.60,18.26)$ & $15.48(13.43,16.75)$ & 0.383 \\
\hline Female & $16.91(14.28,17.86)$ & $14.67(13.09,16,51)$ & 0.029 \\
\hline Duration of diabetes (years) & & & \\
\hline Total & $\mathrm{N} / \mathrm{A}$ & $2.28(1.16,4.05)$ & $\mathrm{N} / \mathrm{A}$ \\
\hline Male & $\mathrm{N} / \mathrm{A}$ & $2.57(1.27,3.92)$ & $N / A$ \\
\hline Female & N/A & $1.96(1.05,4.05)$ & $\mathrm{N} / \mathrm{A}$ \\
\hline HbA1c (\%) & & & \\
\hline Total & $5.60(5.50,5.80)$ & $9.50(7.05,11.45)$ & $<0.001$ \\
\hline Male & $5.50(5.50,5.70)$ & $9.45(6.87,11.72)$ & $<0.001$ \\
\hline Female & $5.60(5.50,5.80)$ & $9.50(7.20,11.20)$ & $<0.001$ \\
\hline BMI (z-score) & & & \\
\hline Total & $2.50(1.96,3.05)$ & $2.47(1.80,3.09)$ & 0.784 \\
\hline Male & $2.96(2.12,3.40)$ & $2.50(1.89,3.33)$ & 0.637 \\
\hline Female & $2.43(1.82,2.94)$ & $2.39(1.77,3.00)$ & 0.991 \\
\hline ACR (mg/mmol Cr) & & & \\
\hline Total & $0.20(0,0.55)$ & $0.71(0.2,2.7)$ & $<0.001$ \\
\hline Male & $0(0,0.14)$ & $0.60(0.16,1.71)$ & 0.001 \\
\hline Female & $0.40(0.16,0.78)$ & $0.84(0.23,3.27)$ & 0.018 \\
\hline \multirow{2}{*}{ eGFR $\left(\mathrm{ml} / \mathrm{min} / 1.73 \mathrm{~m}^{2}\right)$} & & & \\
\hline & $139.40(123.60,153.40)$ & $143.10(131.7,156.07)$ & 0.063 \\
\hline Male & $147.50(136.6,163.8)$ & $154.80(140.25,169.98)$ & 0.685 \\
\hline Female & $130.10(119.58,144.32)$ & $139.40(127.35,150.85)$ & 0.049 \\
\hline \multicolumn{4}{|l|}{$\begin{array}{l}\text { Hypertension (N, \%) } \\
\text { Total }\end{array}$} \\
\hline \multirow{2}{*}{\multicolumn{4}{|c|}{$\begin{array}{l}\text { Total } \\
\text { Male }\end{array}$}} \\
\hline & & & \\
\hline \multicolumn{4}{|c|}{$24 / 52,46.2 \%$} \\
\hline No & $7 / 15,46.7 \%$ & $24 / 52,46.2 \%$ & 1.000 \\
\hline $\mathrm{N} / \mathrm{A}$ & $2 / 15,13.3 \%$ & $4 / 52,7.6 \%$ & \\
\hline Female & $12 / 30,40.0 \%$ & $56 / 107,52.3 \%$ & \\
\hline No & $17 / 30,56.7 \%$ & $48 / 107,44.9 \%$ & 0.295 \\
\hline $\mathrm{N} / \mathrm{A}$ & $1 / 30,3.3 \%$ & $3 / 107,2.8 \%$ & \\
\hline
\end{tabular}


bioRxiv preprint doi: https://doi.org/10.1101/2021.08.23.457385; this version posted August 24, 2021. The copyright holder for this preprint (which was not certified by peer review) is the author/funder, who has granted bioRxiv a license to display the preprint in perpetuity. It is made

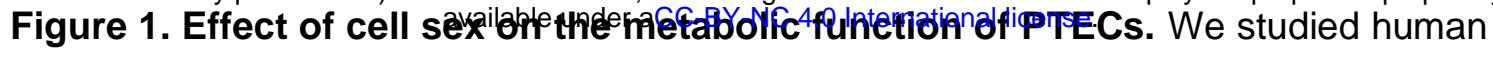

primary PTECs from 4 male and 5 female donors (Lonza) (A). We measured glycolysis as the extracellular acidification rate $(E C A R)$ and oxygen consumption rate $(O C R)$ in male and female PTECs ( $n=3 /$ sex; $n=4-6$ technical replicates/donor) in real-time in a Seahorse XFe96 analyzer. To induce metabolic stress, the following sequence of drugs was injected: $1 \mu \mathrm{M}$ oligomycin, $0.3 \mu \mathrm{M}$ FCCP, 100mM 2-DG, 1mM Rot/AA. Our results suggest a more glycolytic, oxidative and energetic phenotype in male PTECs, as compared to female PTECs (B). Baseline ECAR (C), maximal glycolytic capacity (D), and glycolytic reserve $(\mathrm{E})$ were calculated from the ECAR curves in panel B. Basal respiration $(F)$, ATP-linked respiration $(G)$, maximal respiratory capacity $(H)$, reserve capacity $(\mathrm{I})$, proton leak $(\mathrm{J})$, and non-mitochondrial respiration $(\mathrm{K})$ were calculated from the OCR curves in panel B. Rapid glucose uptake (1h) was assessed in male and female PTECs by employing a luminescence assay (L) ( $n=3$ technical replicates). ${ }^{*} p<0.05$; ${ }^{* * *} p<0.001$. PTECs, proximal tubular epithelial cells; $M$, male; F, female; AUC, area under the curve; ECAR, extracellular acidification rate; OCR, oxygen consumption rate; OLIGO, oligomycin; FCCP, p-trifluoromethoxy carbonyl cyanide phenyl hydrazone; 2-

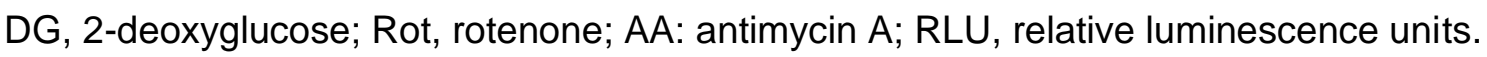


A

\section{\begin{tabular}{|l|l|l|l|}
\hline DONOR & LOT \# & AGE & SEX \\
\hline
\end{tabular} \\ \begin{tabular}{|l|l|l|l}
\hline M-A & 530068 & $57 \mathrm{Y}$ & MALE \\
\hline
\end{tabular} \\ \begin{tabular}{|l|l|l|l|}
\hline M-B & 664995 & $52 Y$ & MALE \\
\hline
\end{tabular} \\ \begin{tabular}{|l|l|l|l|}
\hline M-C & 682573 & $50 Y$ & MALE \\
\hline
\end{tabular} \\ \begin{tabular}{|c|c|c|c|}
\hline M-D & 117405 & $57 Y$ & MALE \\
\hline
\end{tabular}

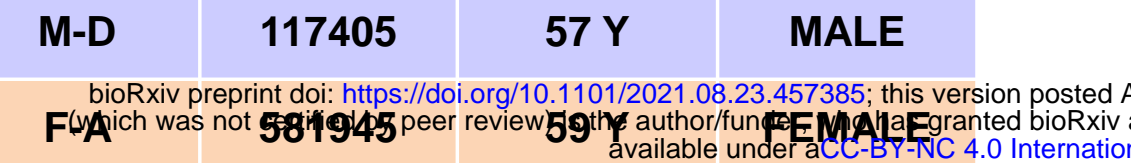 \\ \begin{tabular}{|l|l|l|l|}
\hline F-B & 560580 & $44 \mathrm{Y}$ & FEMALE \\
\hline
\end{tabular} \\ \begin{tabular}{|l|l|l|l|}
\hline F-C & 617045 & $55 Y$ & FEMALE \\
\hline
\end{tabular} \\ \begin{tabular}{|l|l|l|l|} 
F-D & 635750 & $52 Y$ & FEMALE \\
\hline
\end{tabular} \\ \begin{tabular}{|l|l|l|l|}
\hline F-E & 114340 & $59 \mathrm{Y}$ & FEMALE \\
\hline
\end{tabular}}

C

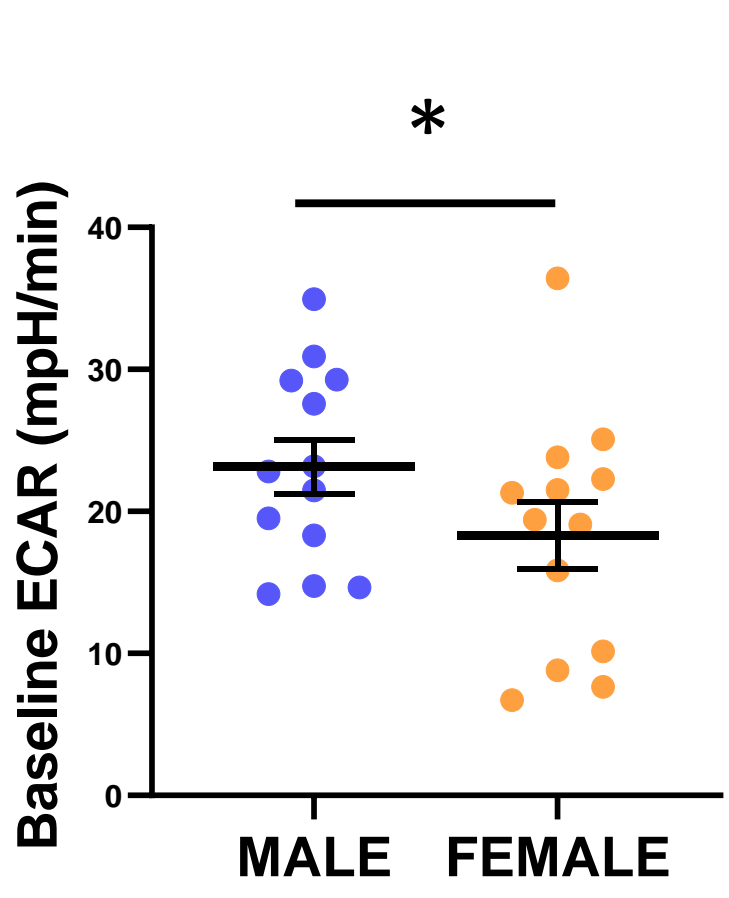

D

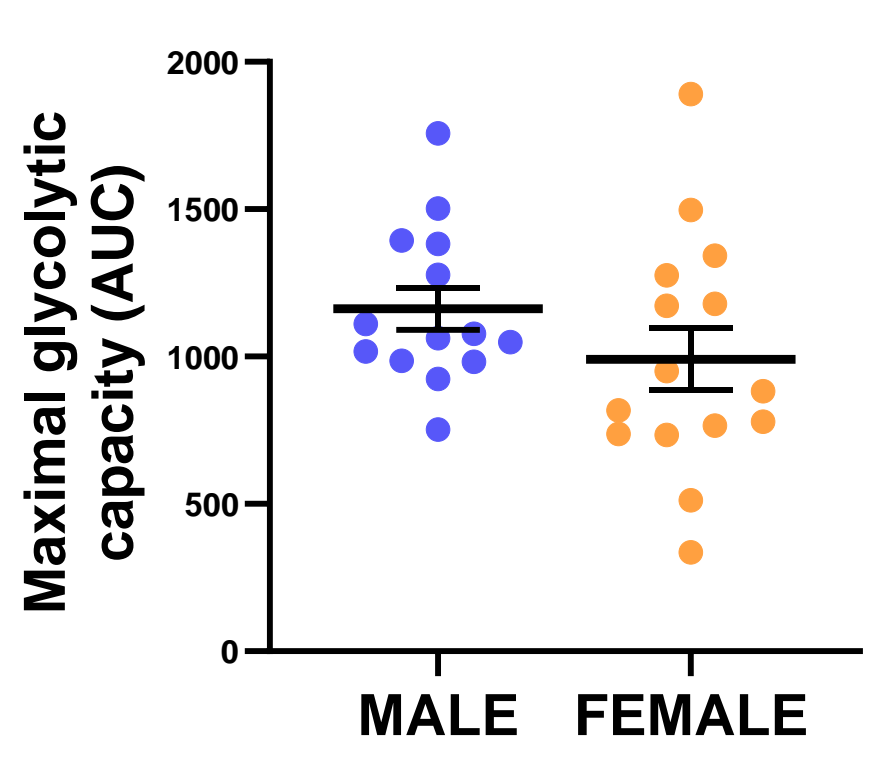

E

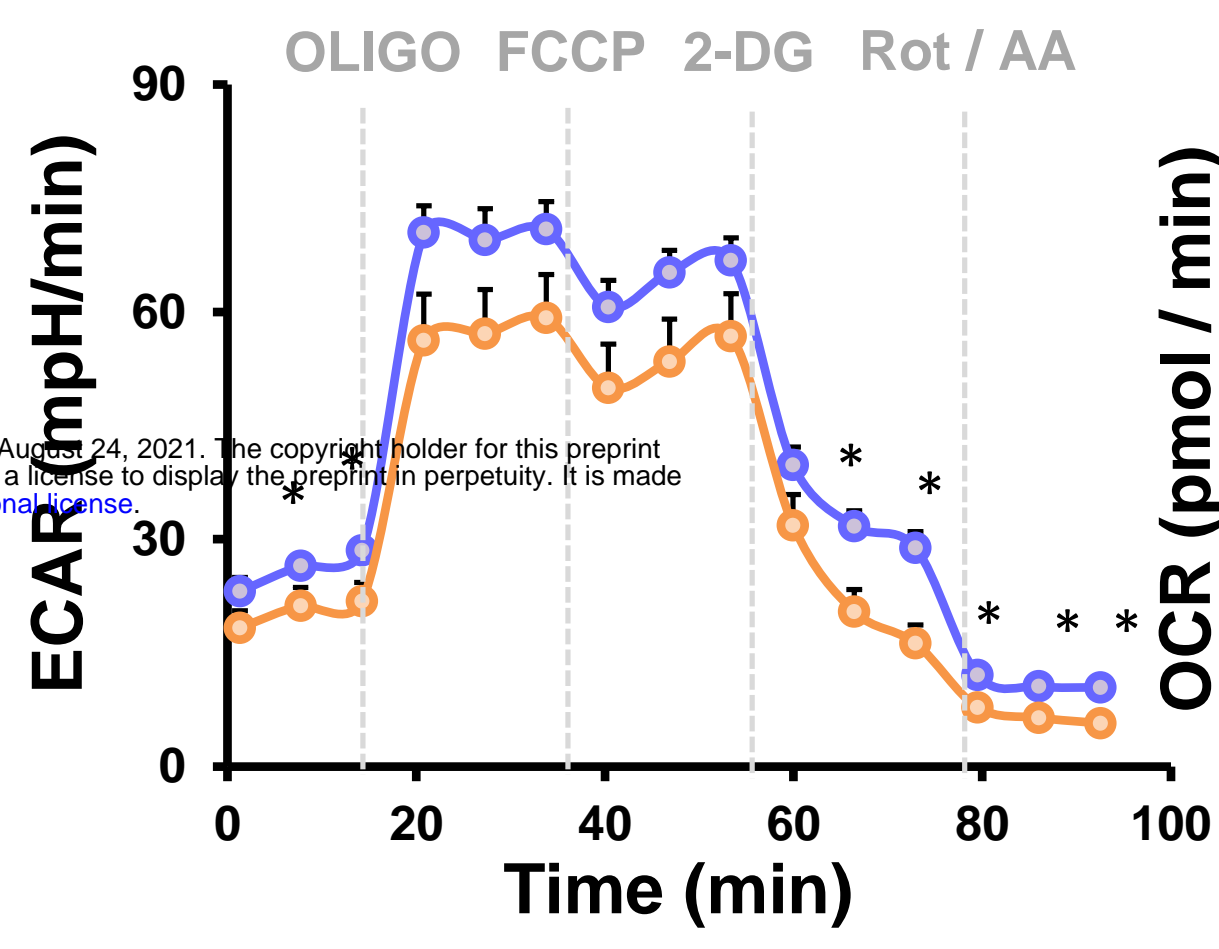

$\mathbf{F}$
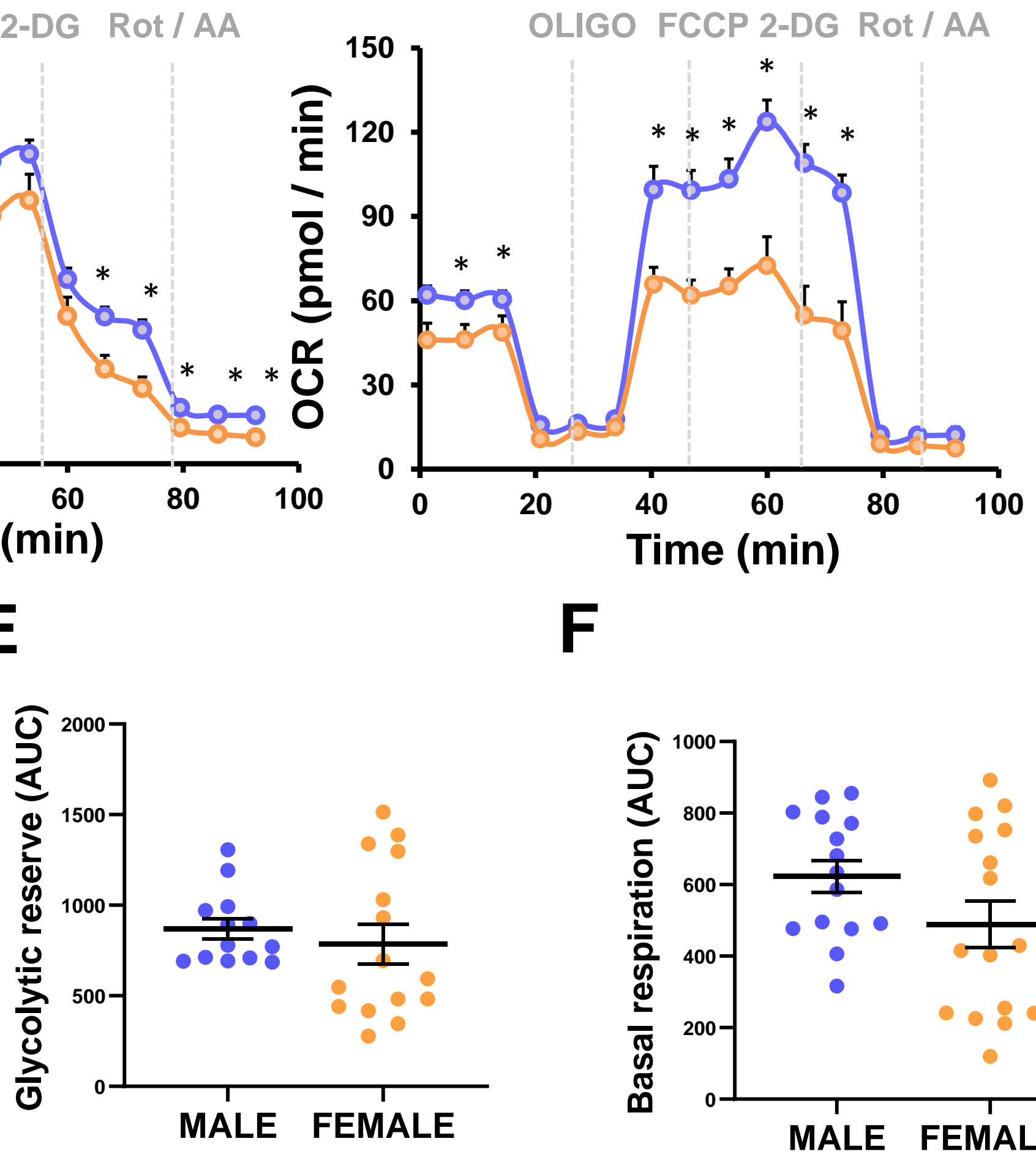

J

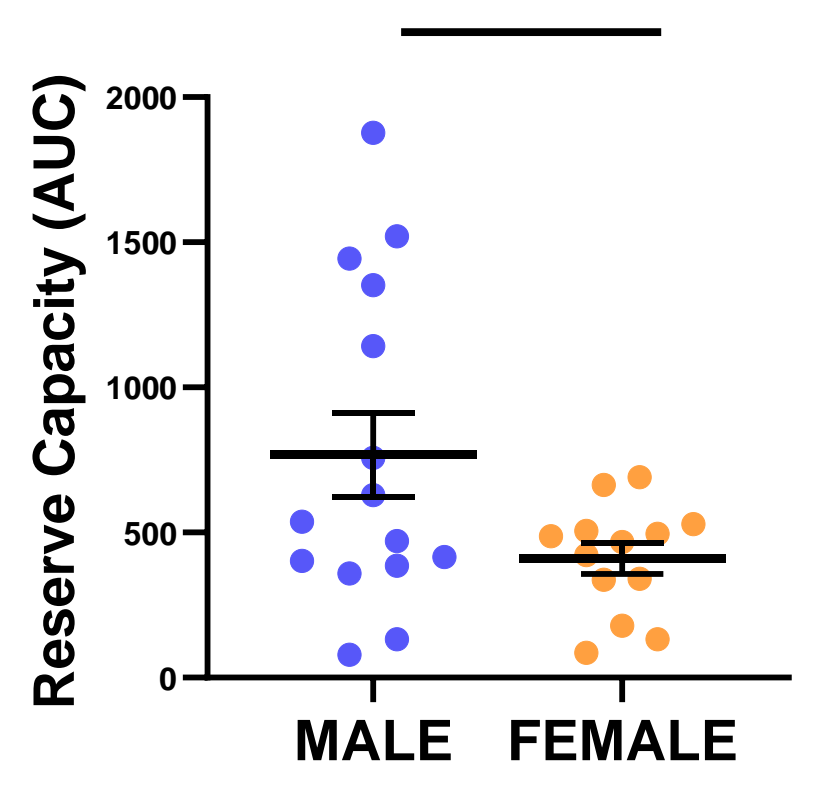

FIGURE 1

○- MALE PTECs - $\cdot$ FEMALE PTECs

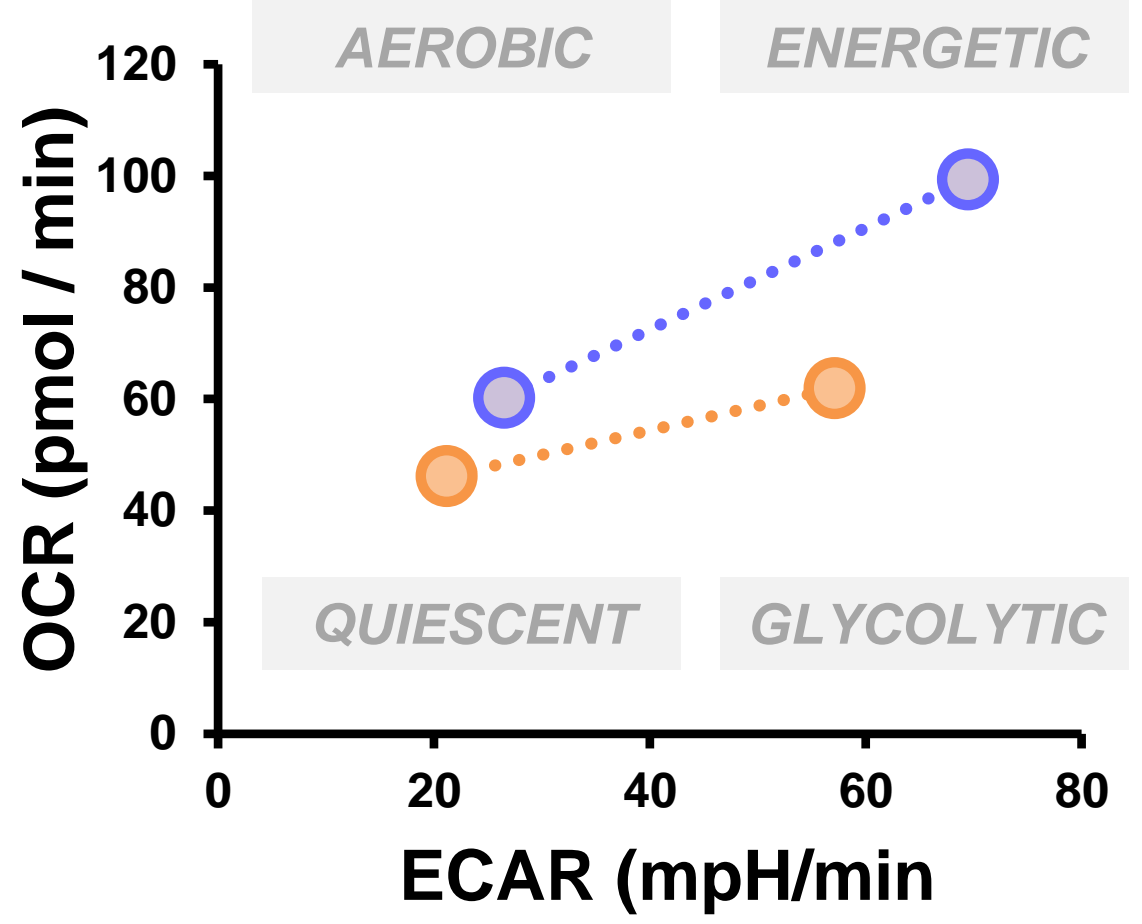

G
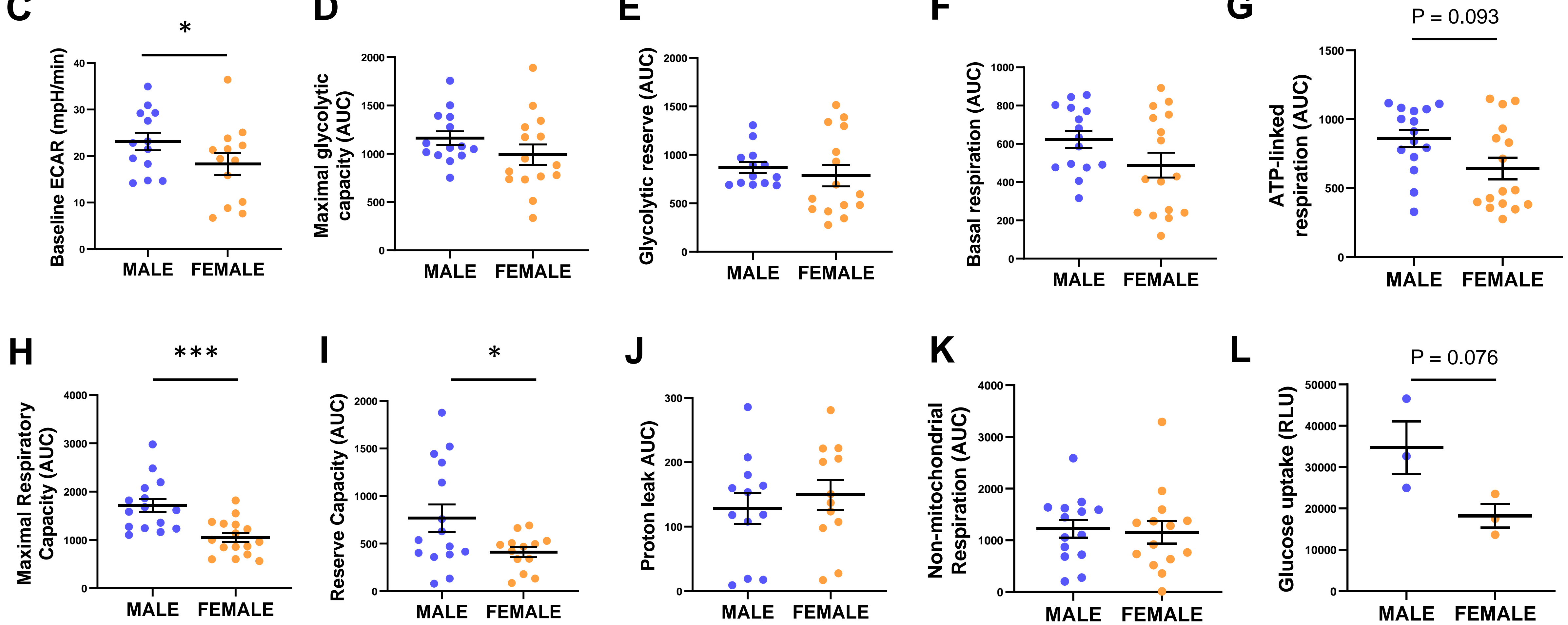

K

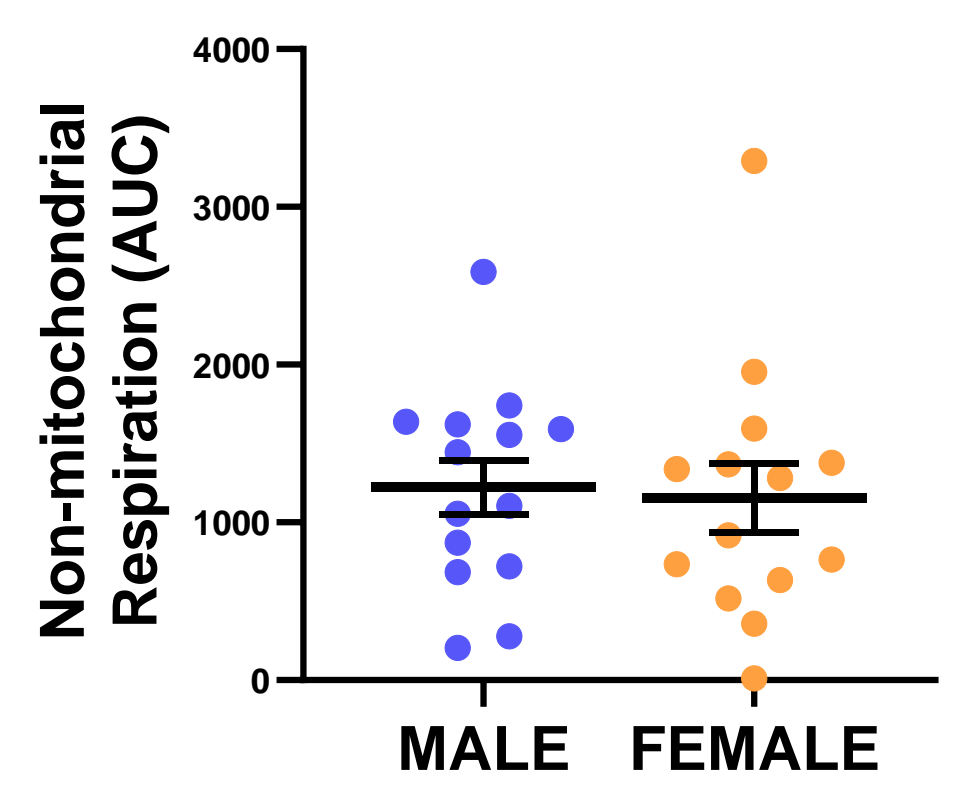

$\mathbf{L}$

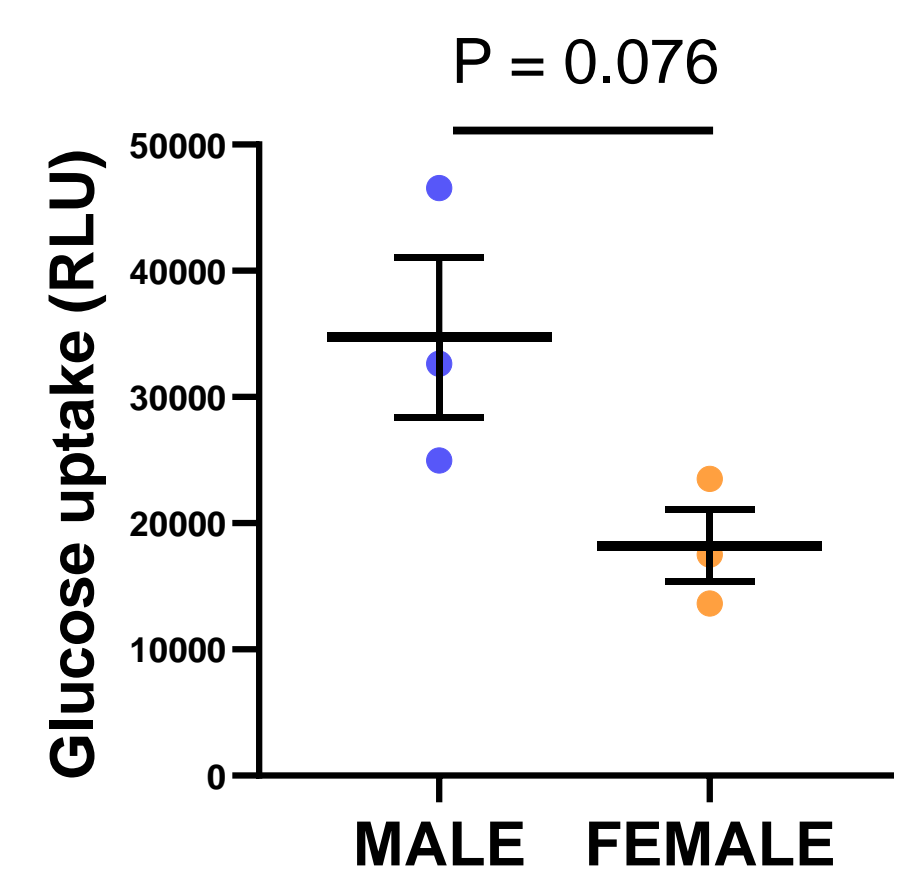


bioRxiv preprint doi: https://doi.org/10.1101/2021.08.23.457385; this version posted August 24, 2021. The copyright holder for this preprint (which was not certified by peer review) is the author/funder, who has granted bioRxiv a license to display the preprint in perpetuity. It is made

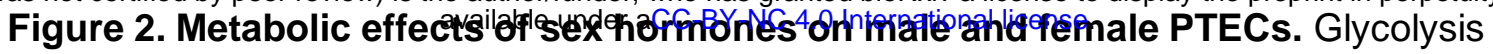

was assessed in male and female PTECs by measuring the extracellular acidification rate (ECAR) after stimulation with ethanol (CONT), 100nM DHT or 100nM EST for $16 \mathrm{~h}$ $(n=3 /$ sex; $n=4-6 /$ treatment). Oxygen consumption rate $(O C R)$ was also monitored $(A)$. To induce metabolic stress, the following sequence of drugs was injected: $1 \mu \mathrm{M}$ oligomycin, $0.3 \mu \mathrm{M}$ FCCP, 100mM 2-DG, 1 mM Rot/AA. The metabolic phenotype of male and female PTECs exposed to sex hormones was evaluated by plotting ECAR in the X axis, and OCR in the $\mathrm{Y}$ axis (B). Glucose levels in the media were assessed using Accucheck Aviva Nano strips in CONT-, DHT-, and EST-treated male and female PTECs at 16h (C), 24h (D), and 48h (E) ( $\mathrm{n}=2 /$ sex; $n=4-6 /$ treatment). After $16 \mathrm{~h}$ of hormone exposure, intracellular levels of superoxide ion were measured to assess oxidative stress $(F)$ ( $n=3 /$ sex; $n=4-6 /$ treatment), and surface levels of phosphatidylserine were measured to assess early apoptosis (G) ( $n=2 /$ sex; $n=4-6 /$ treatment). ${ }^{*} p<0.05 ;{ }^{* *} p<0.01$; ${ }^{* * *} \mathrm{p}<0.001$. PTECs, proximal tubular epithelial cells; CONT, control; DHT, dihydrotestosterone; EST, 17ß-estradiol; ECAR, extracellular acidification rate; OCR, oxygen consumption rate; FCCP, p-trifluoromethoxy carbonyl cyanide phenyl hydrazone; 2-DG, 2-deoxyglucose; Rot, rotenone; AA: antimycin A; RFU, relative fluorescence units; PS, phosphatidylserine. 
$\mathbf{A}$
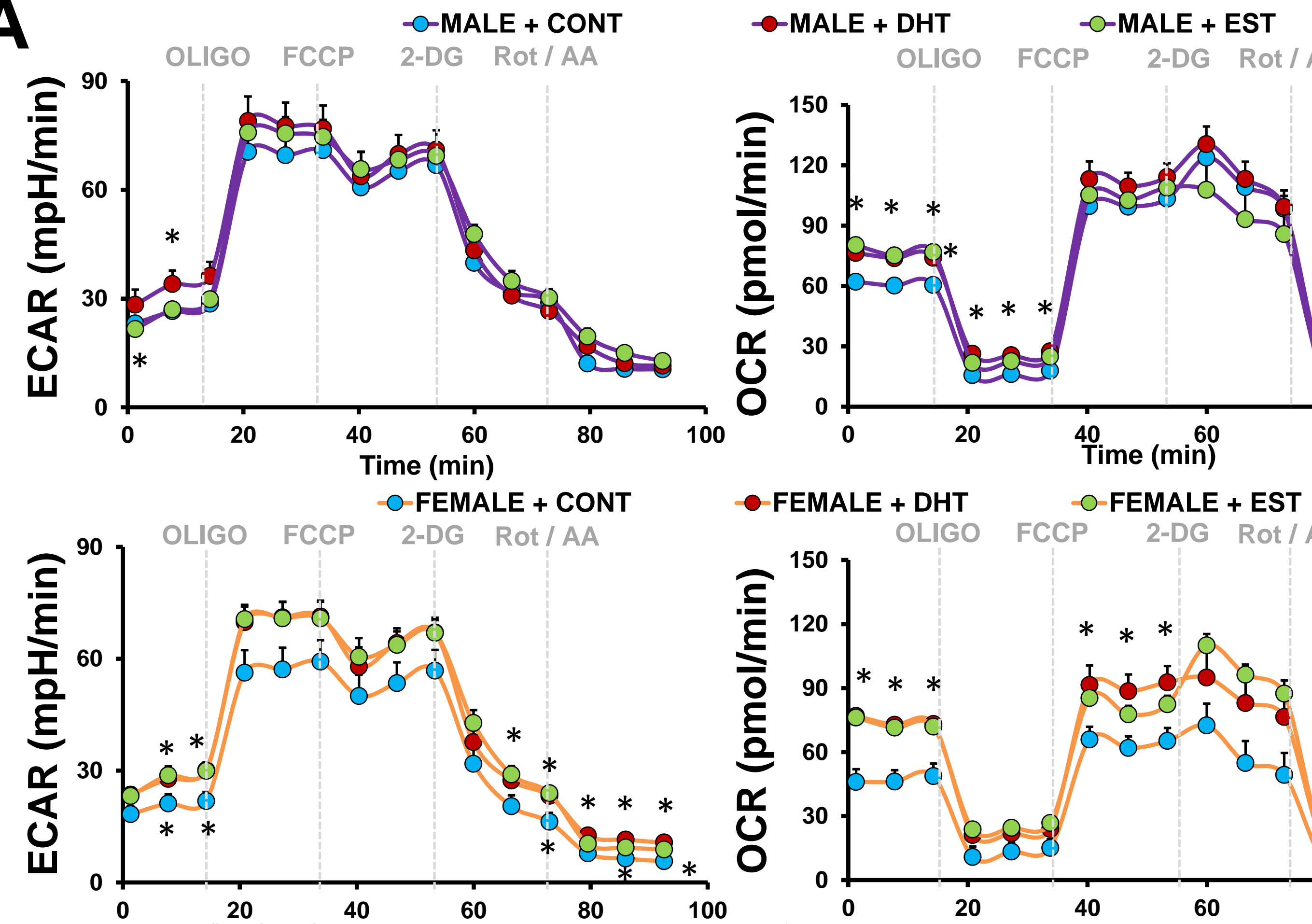

O-FEMALE + DHT O-FEMALE + EST
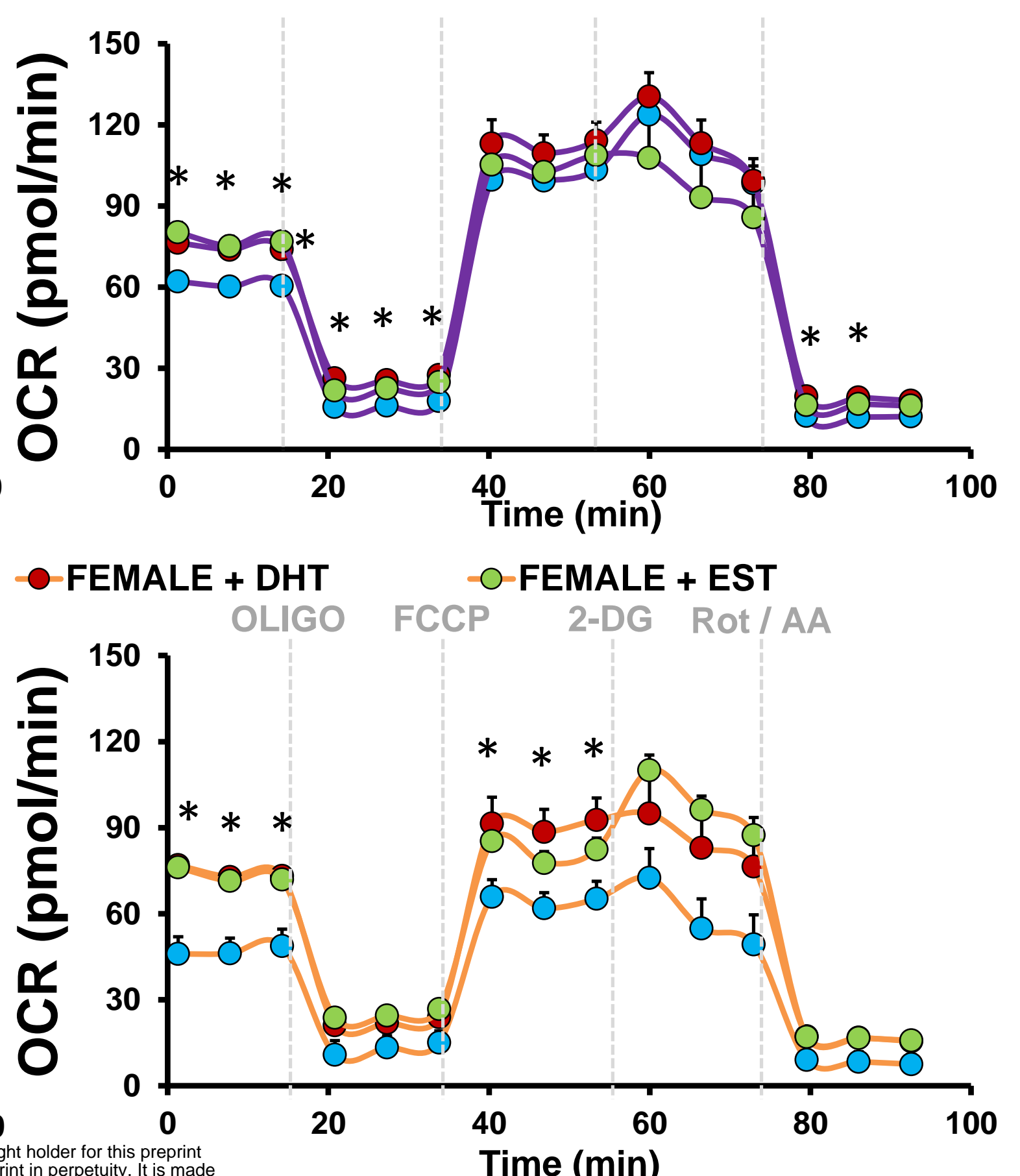

C
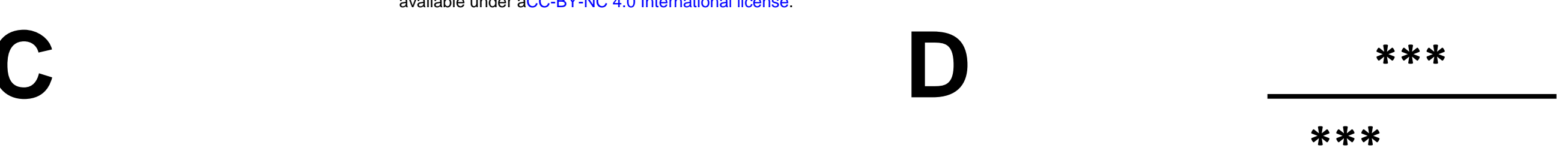

F

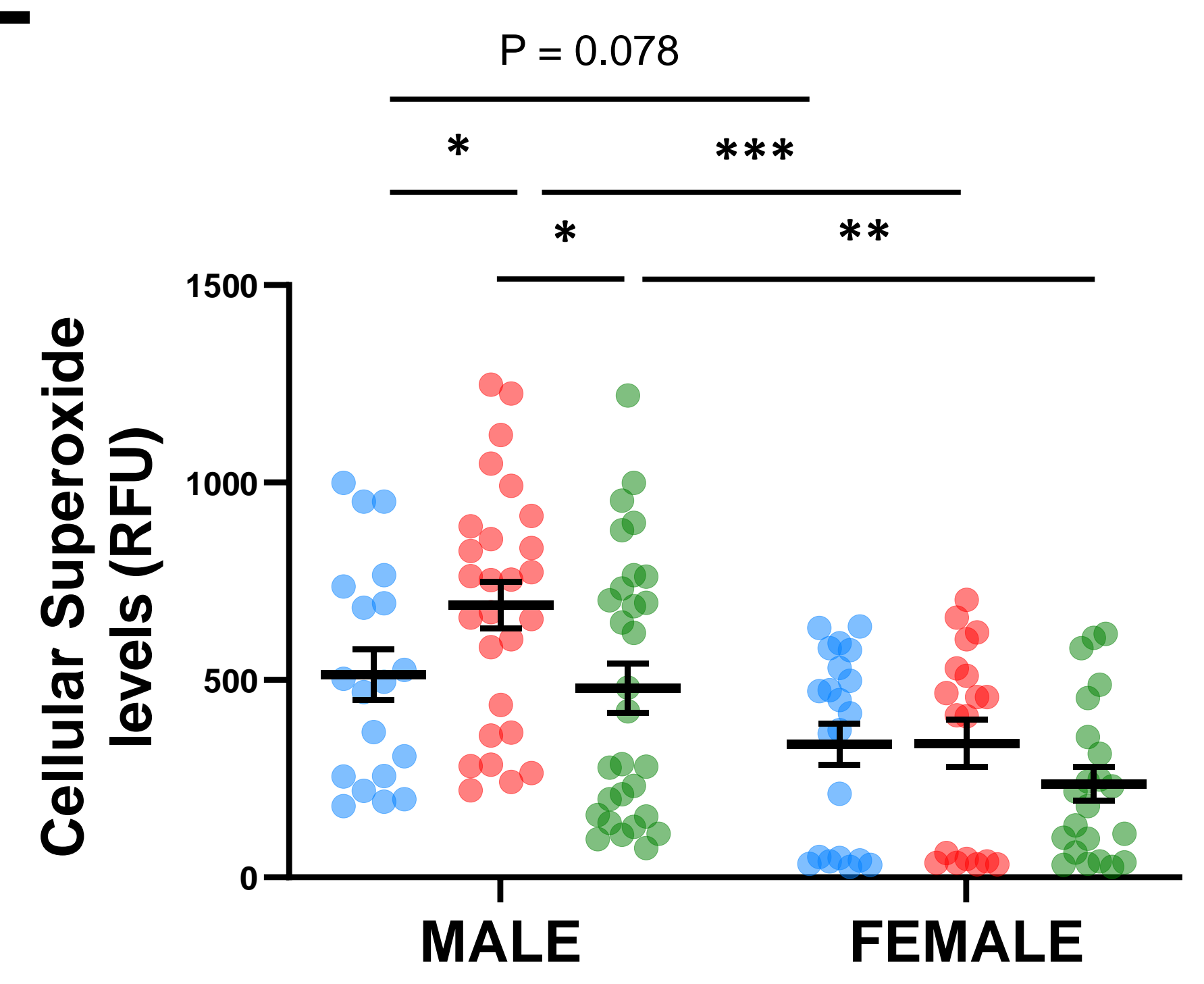

B

FIGURE 2
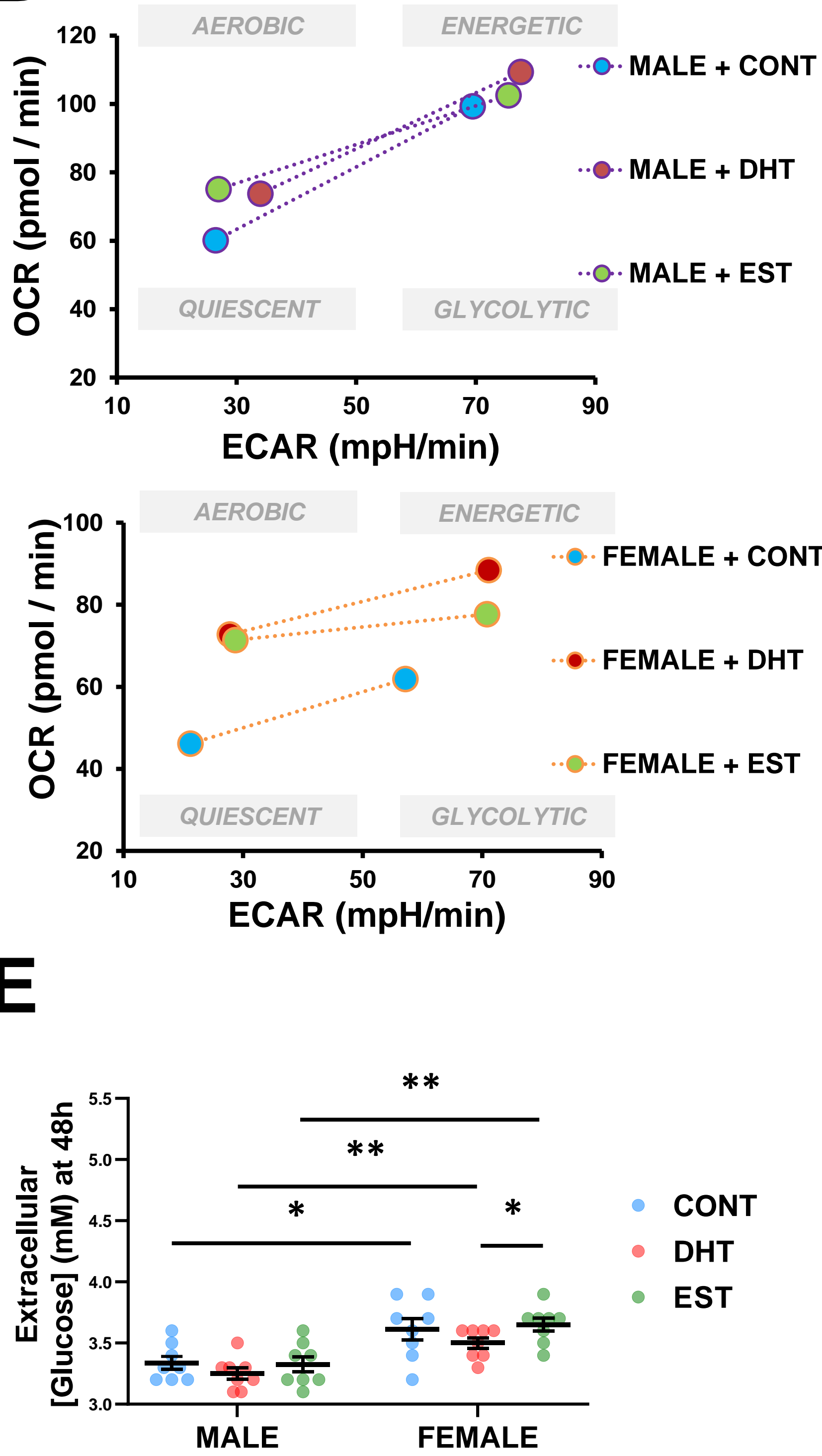

G

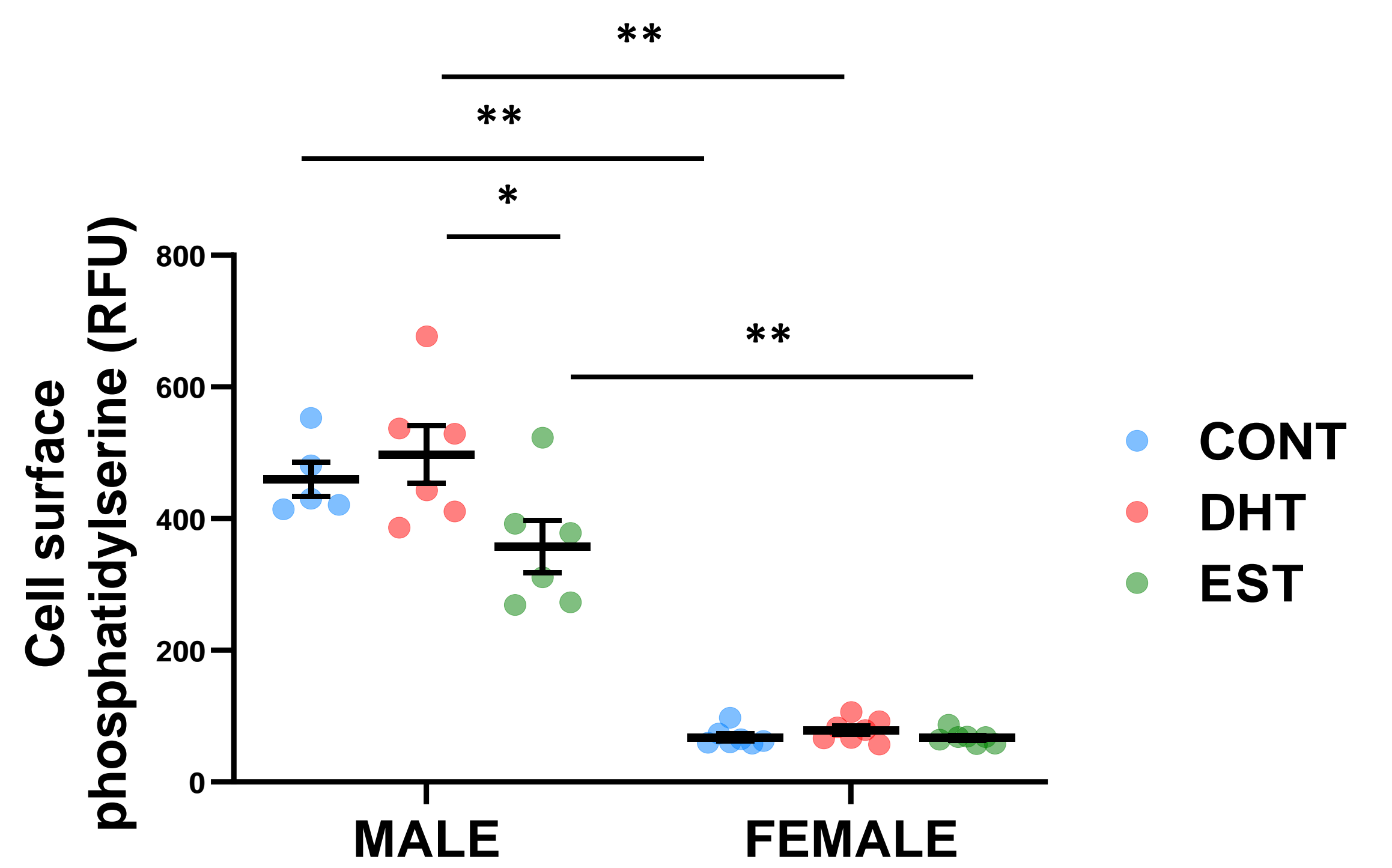


bioRxiv preprint doi: https://doi.org/10.1101/2021.08.23.457385; this version posted August 24, 2021. The copyright holder for this preprint (which was not certified by peer review) is the author/funder, who has granted bioRxiv a license to display the preprint in perpetuity. It is made

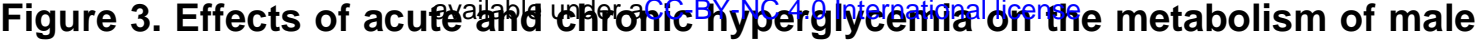

and female PTECs. ECAR and OCR were monitored to assess glycolysis and mitochondrial function, respectively, in male and female PTECs at baseline and after $2 \mathrm{~h}$, $48 \mathrm{~h}$, and $96 \mathrm{~h}$ of exposure to hyperglycemia ( $25 \mathrm{mM}$ glucose); $(\mathrm{n}=2 / \mathrm{sex} ; \mathrm{n}=4-6 /$ time point) (A). To induce metabolic stress, the following sequence of drugs was injected: $1 \mu \mathrm{M}$

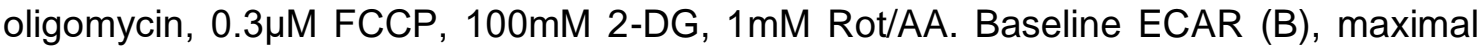
glycolytic capacity (C), and glycolytic reserve (D) were calculated from the ECAR curves in panel A. Baseline OCR (E), maximal respiratory capacity (F), ATP-linked respiration $(G)$, reserve respiratory capacity $(H)$, and non-mitochondrial respiration (I) were calculated from the OCR curves in panel A. The evolution of the metabolic phenotype of male and female PTECs during hyperglycemia was visualized by plotting ECAR on the $X$ axis and OCR on the $Y$ axis $(\mathrm{J})$. The intracellular levels of ATP were also measured (K). ${ }^{*} \mathrm{p}<0.05 ;{ }^{* *} \mathrm{p}<0.01 ;{ }^{* * *} \mathrm{p}<0.001$. PTECs, proximal tubular epithelial cells; AUC, area under the curve; ECAR, extracellular acidification rate; OCR, oxygen consumption rate; FCCP, p-trifluoromethoxy carbonyl cyanide phenyl hydrazone; 2-DG, 2-deoxyglucose; Rot, rotenone; AA: antimycin A. 
bioRxiv preprint doi: https://doi.org/10.1101/2021.08.23.457385; this version posted August 24, 2021. The copyright holder for this preprint (which was not certified by peer review) is the author/funder, who has granted bioRxiv a license to display the preprint in perpetuity. It is made

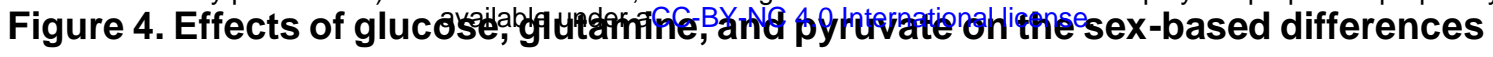

in the metabolic function of PTECs. Oxygen consumption rate (OCR) and glycolysis (ECAR) were measured in male and female PTECs exposed to glucose-only (A-C), glutamine-only (D-F) or pyruvate-only (G-I) conditions for $1 \mathrm{~h}(\mathrm{n}=2-4)$. To induce metabolic stress, the following sequence of drugs was injected: $1 \mu \mathrm{M}$ oligomycin, $0.3 \mu \mathrm{M}$ FCCP, 100mM 2-DG, 1 1 M Rot/AA. Basal respiration, ATP-linked respiration, maximal respiratory capacity, and reserve respiratory capacity were calculated from the OCR curves. Basal glycolysis, maximal glycolytic capacity, and glycolytic reserve were calculated from the ECAR curves. ${ }^{*} p<0.05 ;{ }^{* *} p<0.01 ;{ }^{* *} p<0.001$. PTECs, proximal tubular epithelial cells; Gluc, glucose; Gln, glutamine; Pyr, pyruvate; $\alpha-K G$, alphaketoglutarate; TCA, tricarboxylic acid; AUC, area under the curve; ECAR, extracellular acidification rate; OCR, oxygen consumption rate; FCCP, p-trifluoromethoxy carbonyl cyanide phenyl hydrazone; 2-DG, 2-deoxyglucose; Rot, rotenone; AA: antimycin A. Illustrations in panels $A, D$, and $G$ were created with BioRender.com. 
A

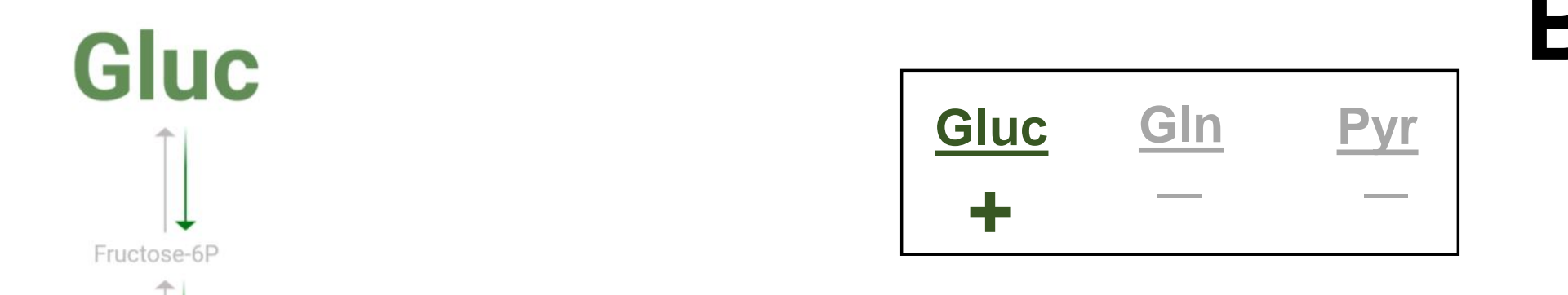

$\rightarrow$-FeMALE PTECs $\rightarrow$ MALE PTECs
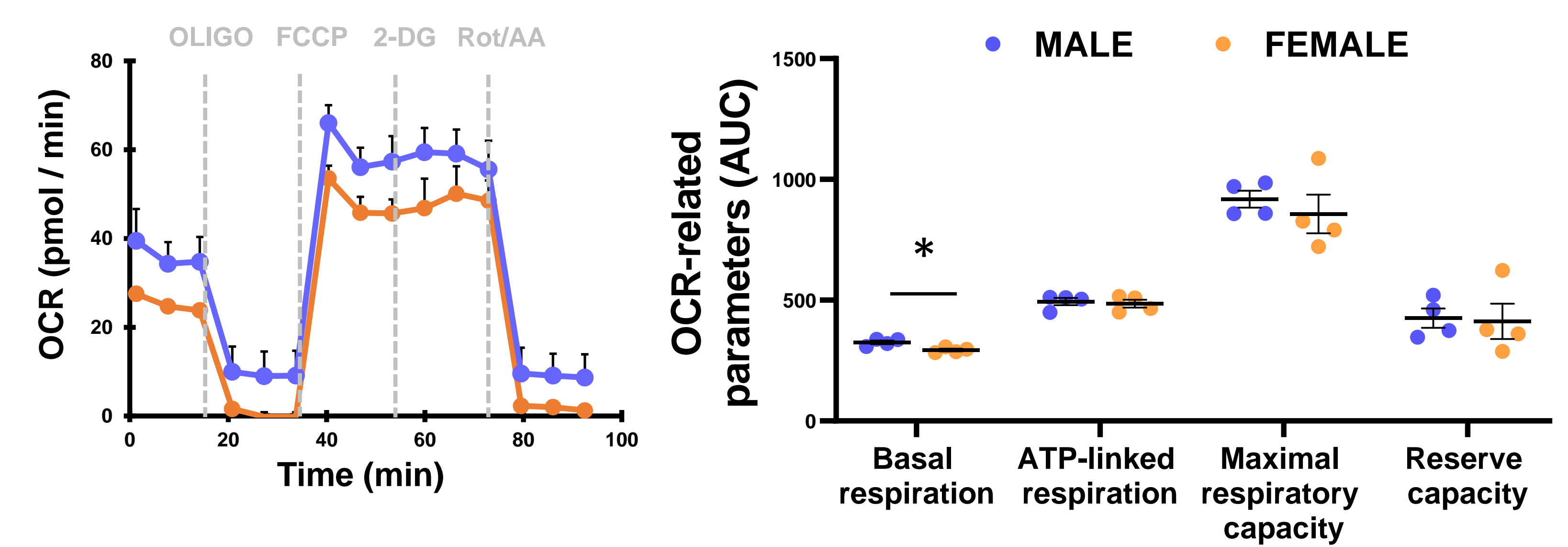

E

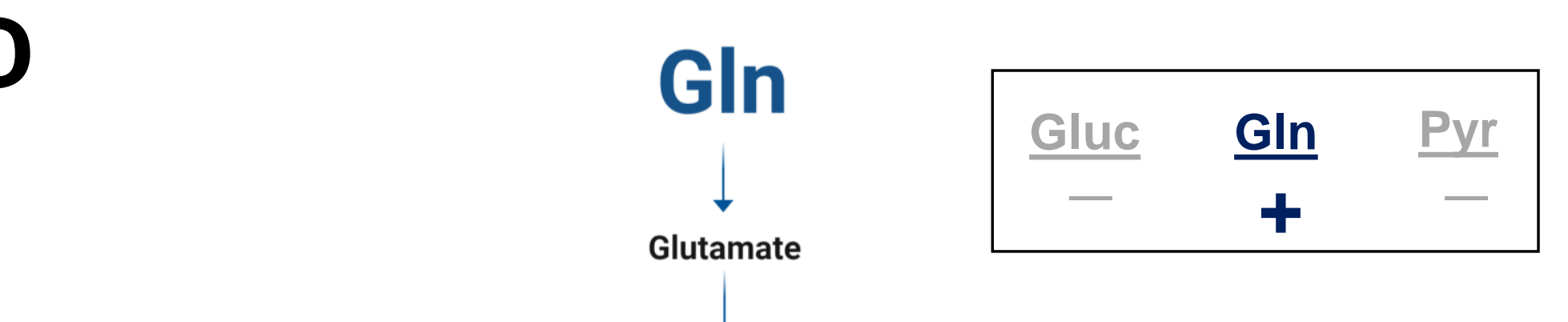

D

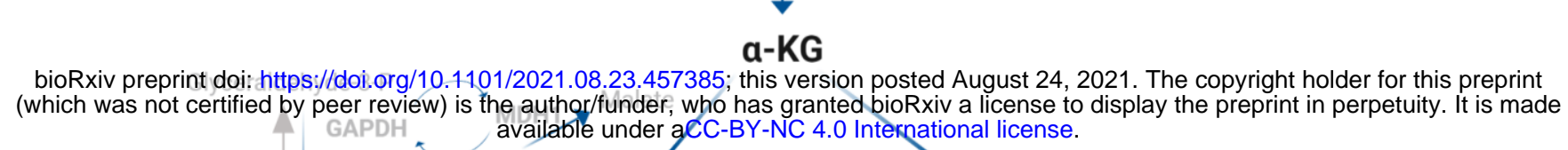

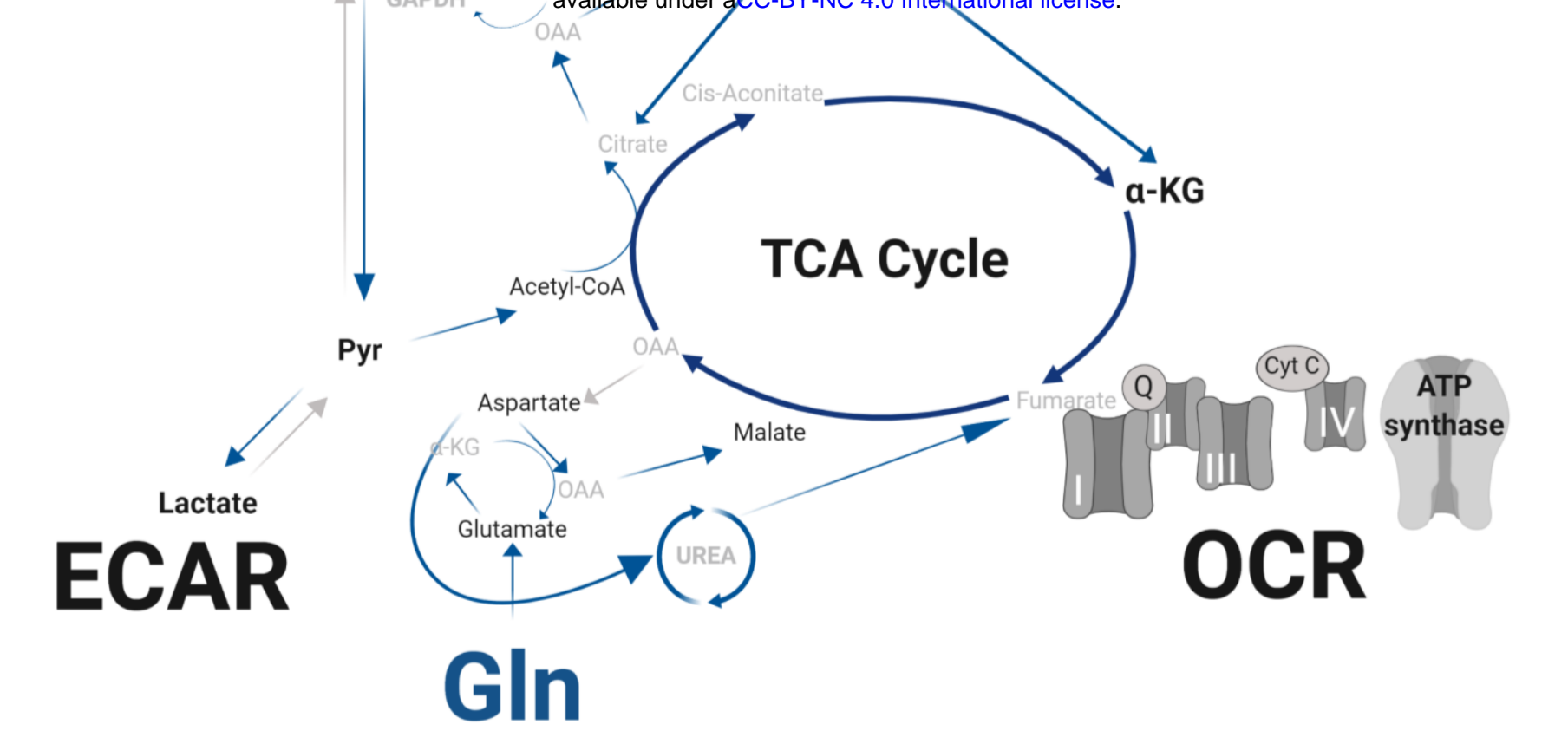

G
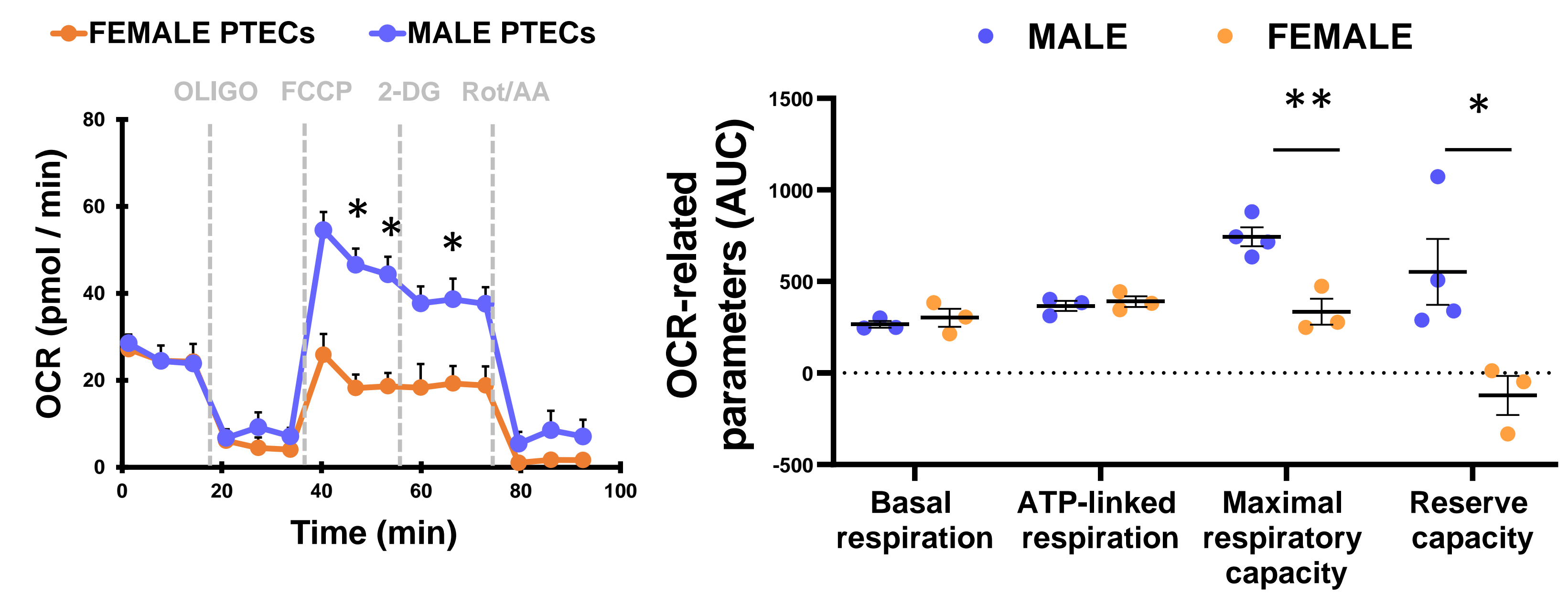

H

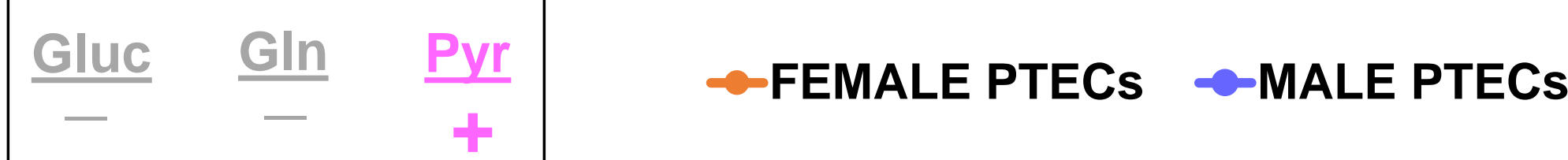

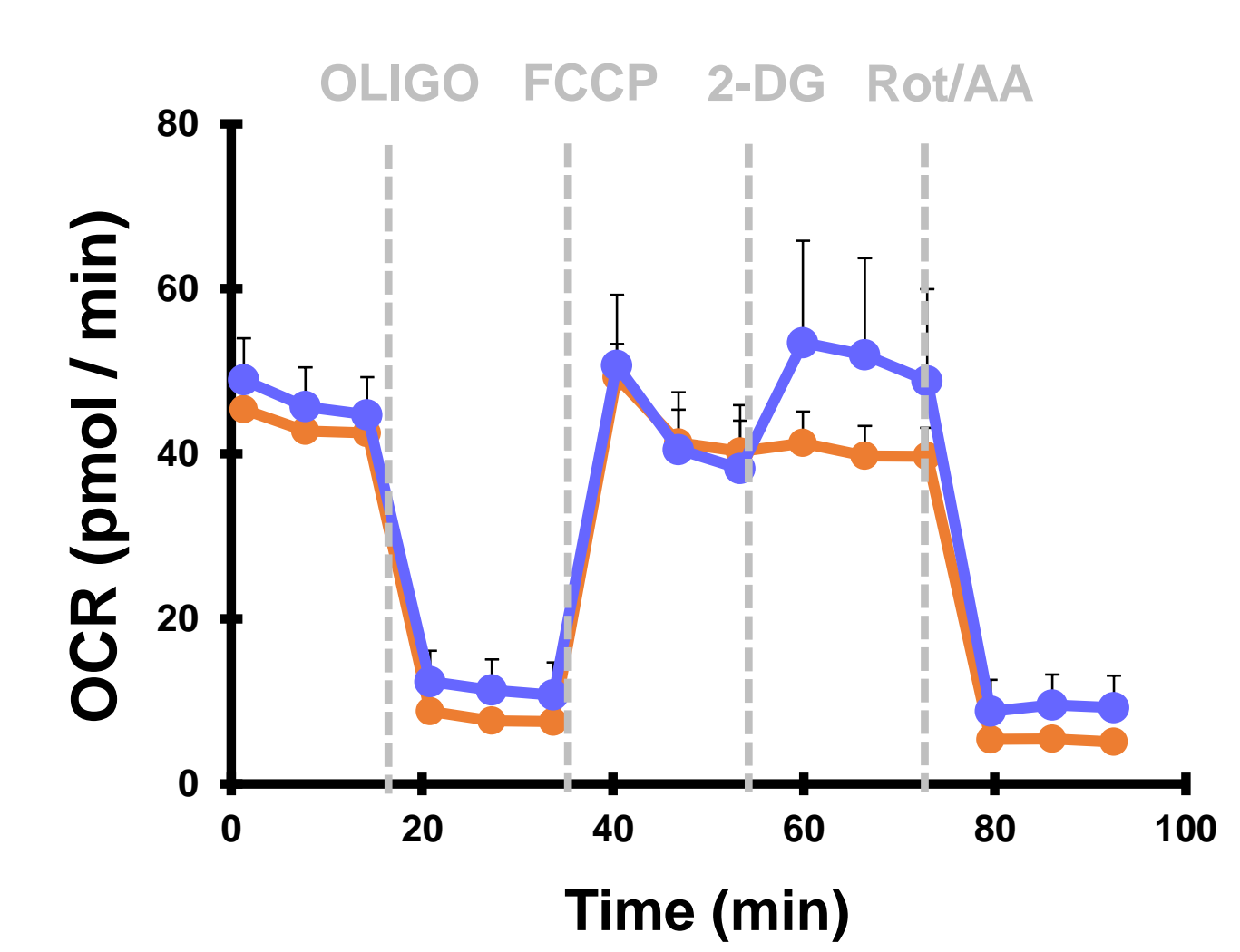

- MALE - FEMALE

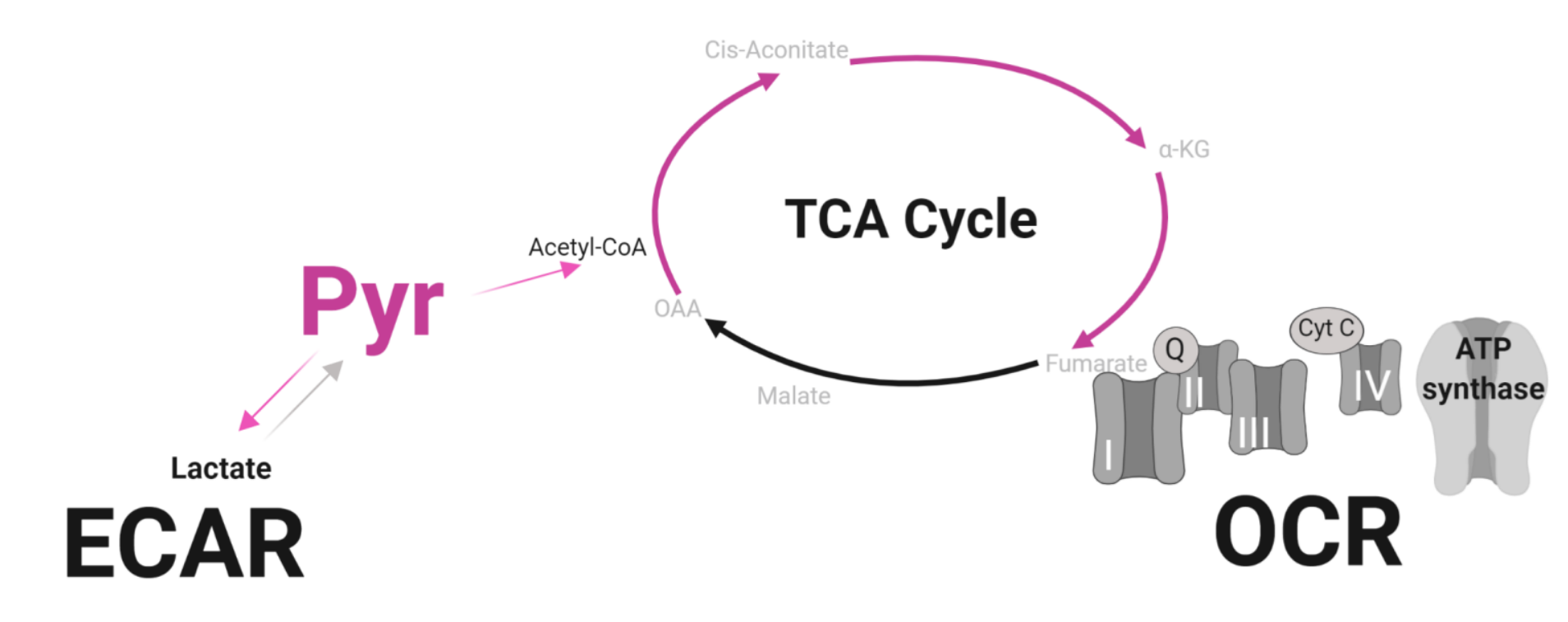

-FEMALE PTECs $\leadsto$-MALE PTECs
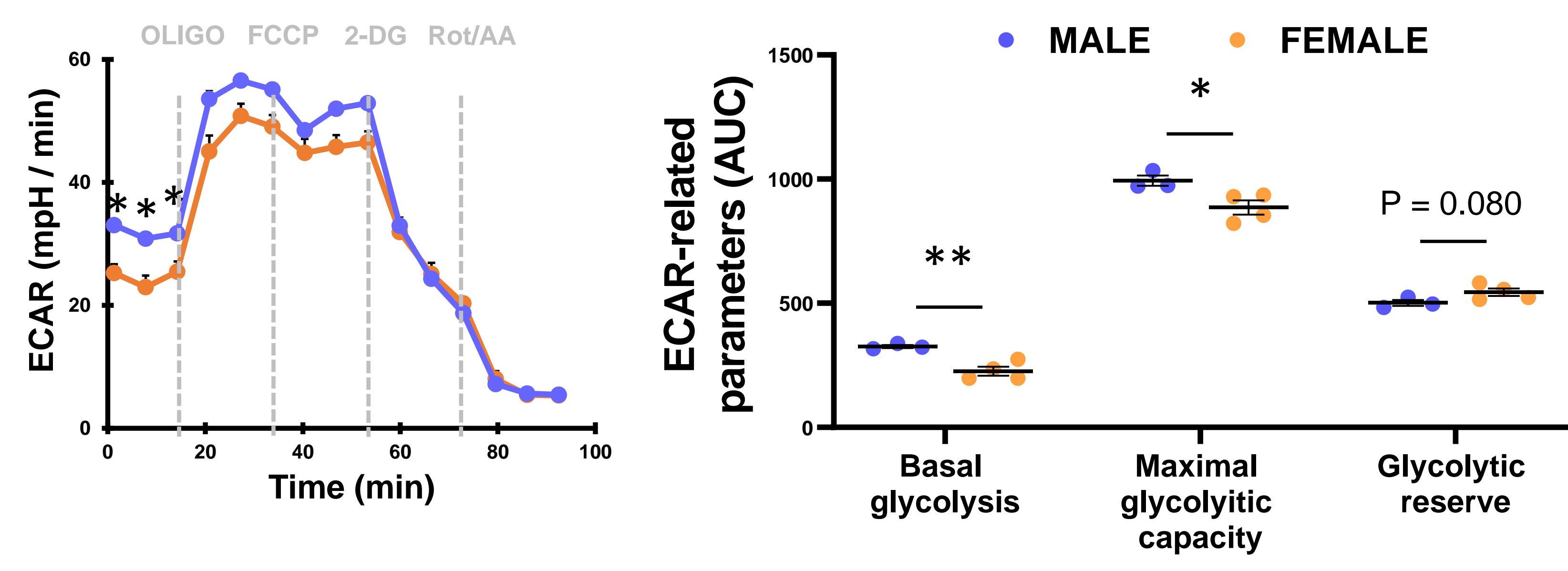

F

-FEMALE PTECs $\quad$ MALE PTECs

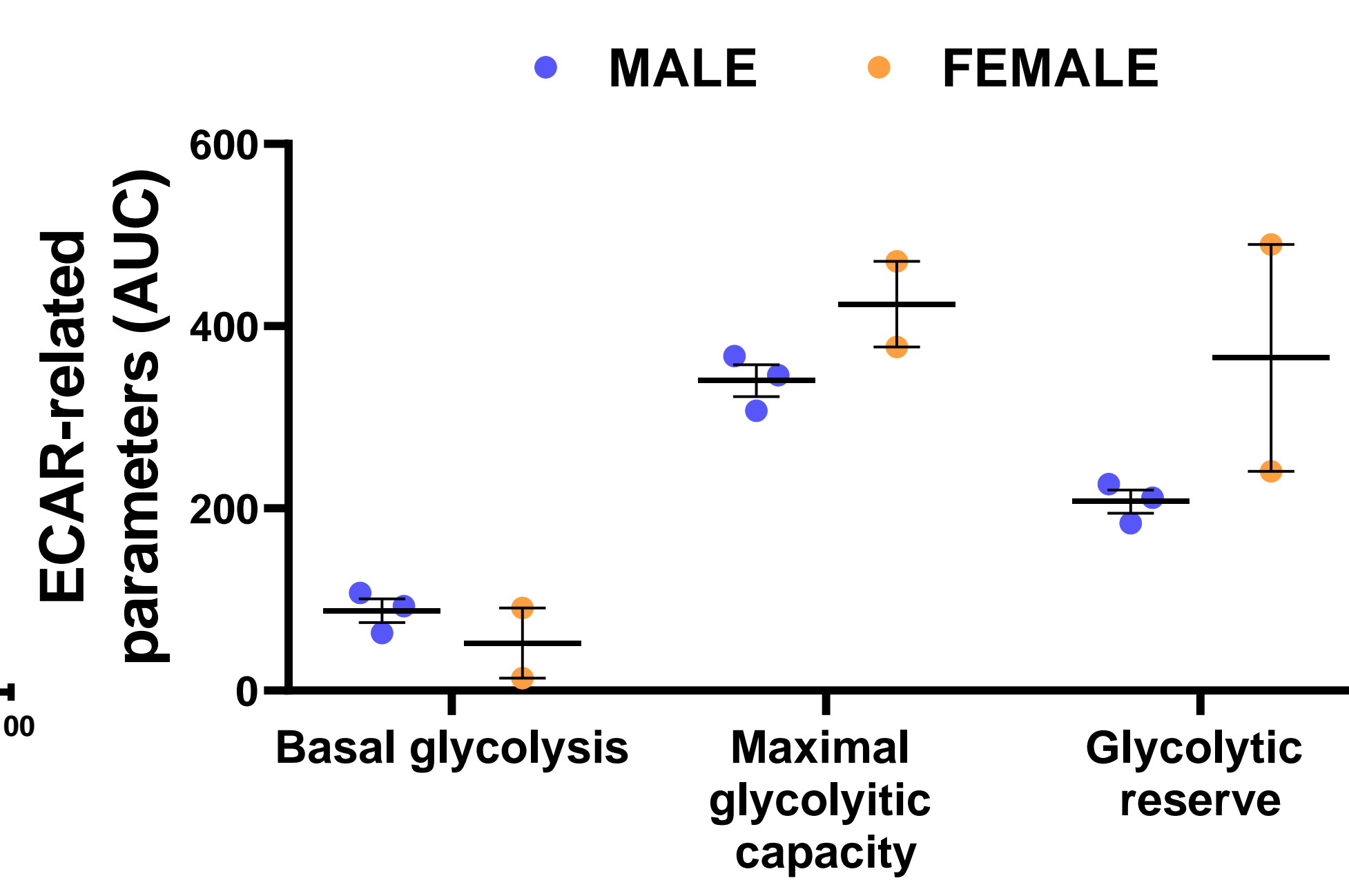

I

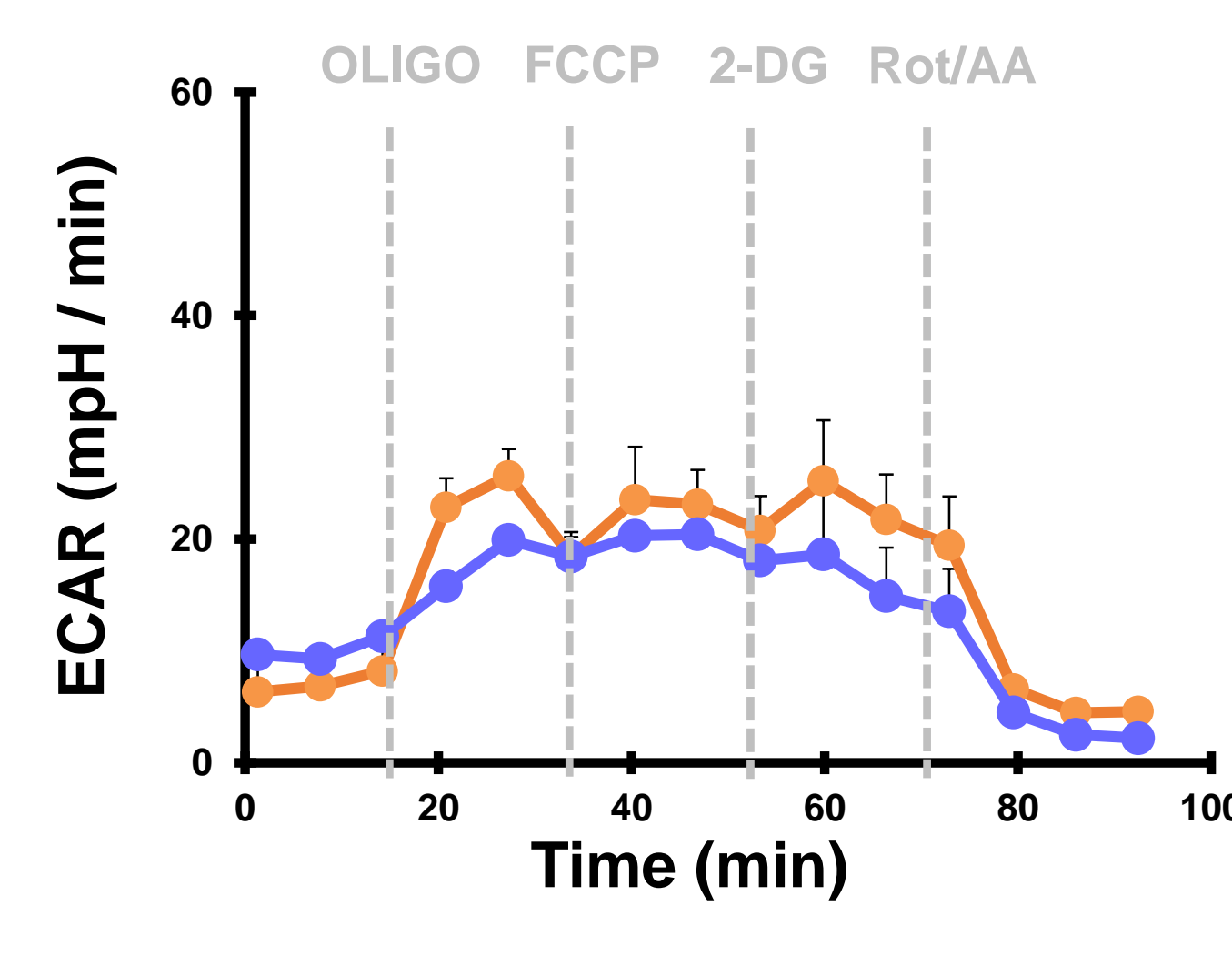

$\rightarrow$ FEMALE PTECs $\rightarrow$ MALE PTECs

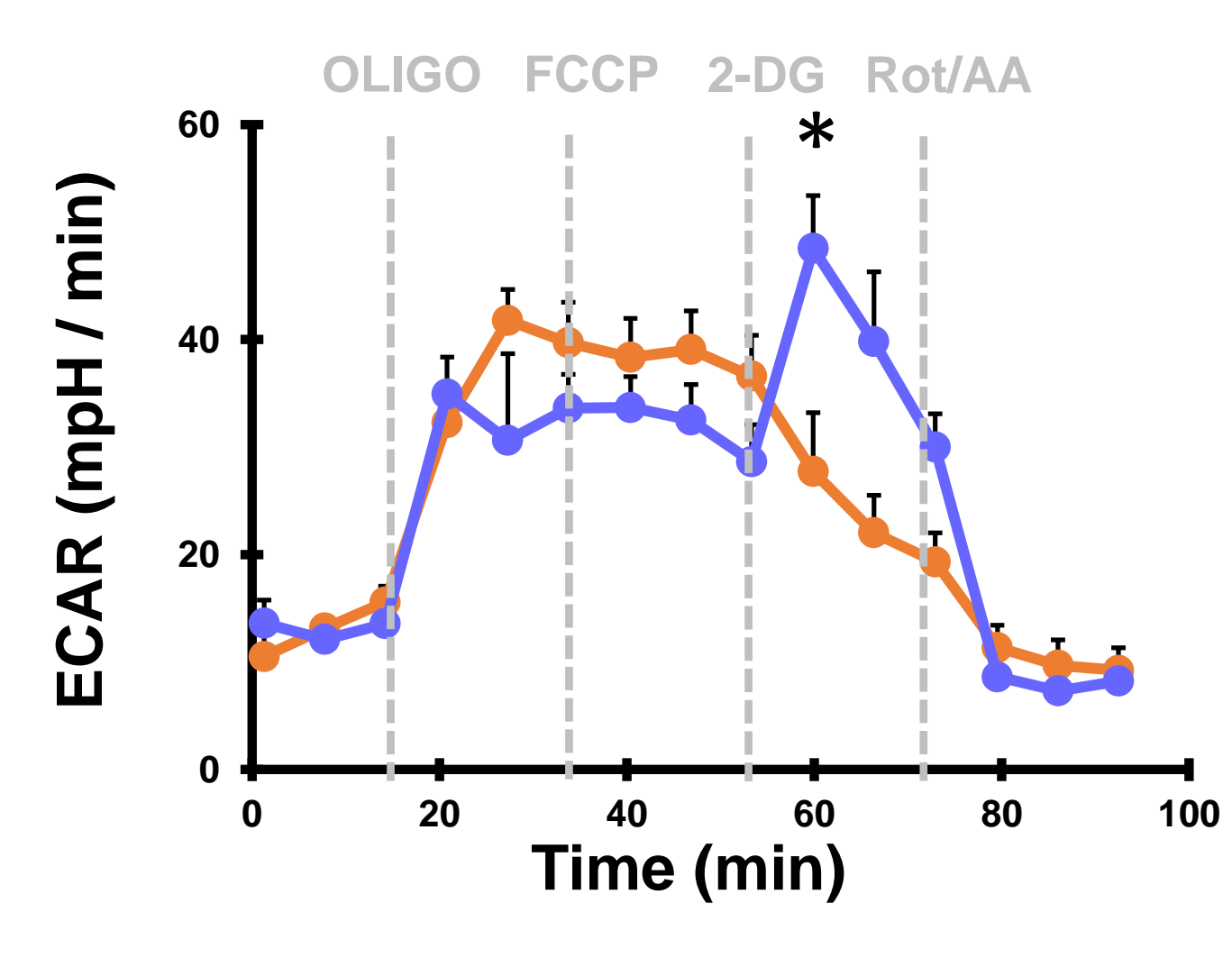

- MALE FEMALE

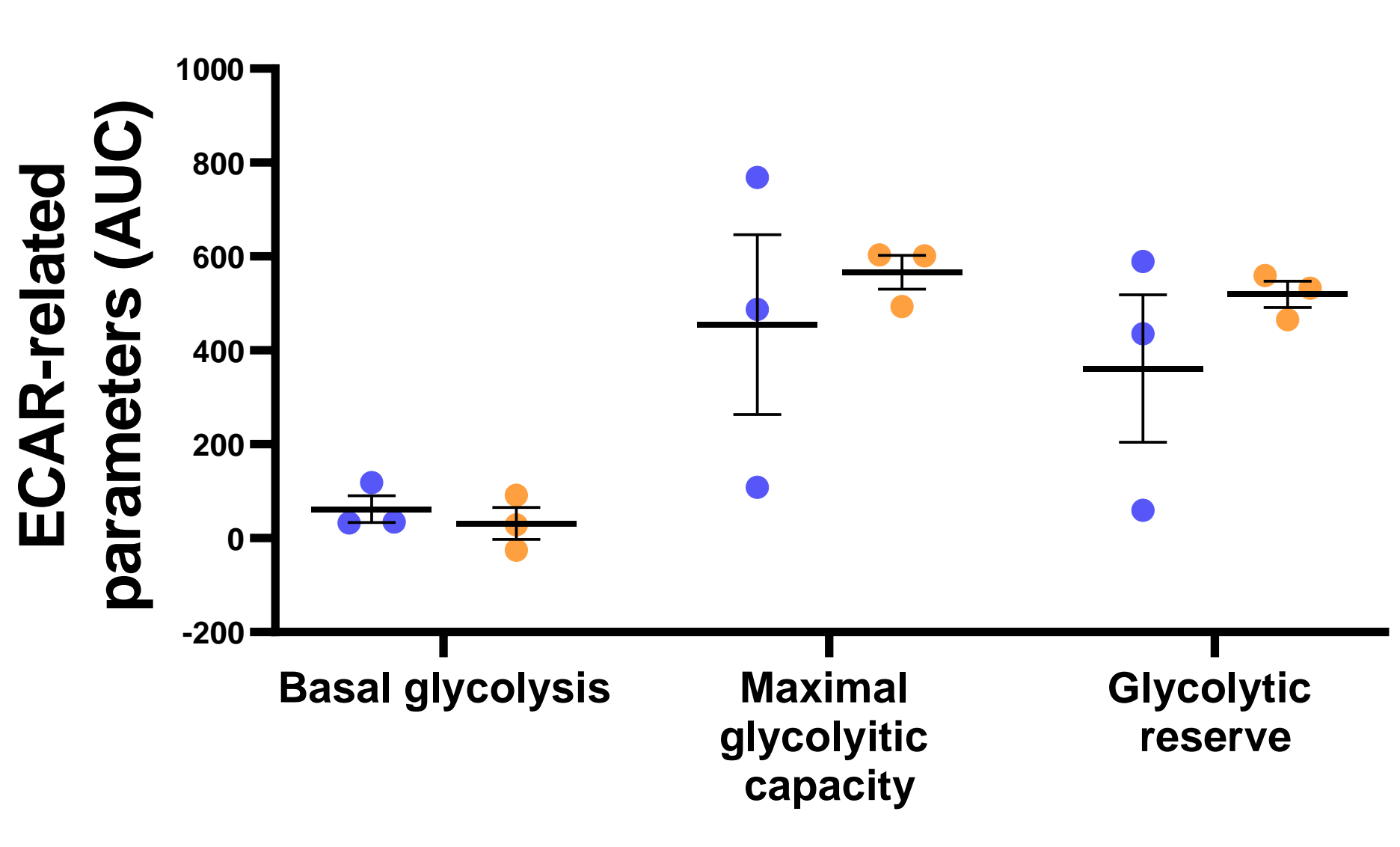


bioRxiv preprint doi: https://doi.org/10.1101/2021.08.23.457385; this version posted August 24, 2021. The copyright holder for this preprint (which was not certified by peer review) is the author/funder, who has granted bioRxiv a license to display the preprint in perpetuity. It is made

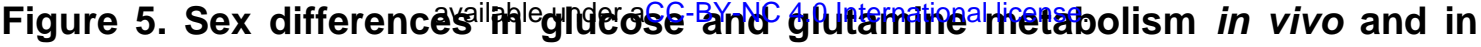

humans. Glucose can generate lactate via glycolysis, while glutamine can be converted to glutamate, which can enter the TCA cycle (A). Glucose, lactate, glutamine and glutamate were measured using a BioProfile ${ }^{\circledR}$ FLEX2 ${ }^{\text {TM }}$ Analyzer in the plasma of male and female type 1 diabetic (Akita) mice, and their healthy wild-type littermates (B). Two different age groups were studied: 8 weeks (C), and 16 weeks (D) ( $n=3-7$ animals/group). Sex differences in glucose, lactate, glutamine, and glutamate levels were also examined in the serum of men without T2DM $(n=15)$, women without T2DM $(n=27)$, men with T2DM $(n=47)$, and women with T2DM $(n=91)(E-F)$. Data normalization was performed to correct variation emerging from inter-day tuning differences on instrument performance. Each compound was corrected in run-day blocks by registering the medians to 1.00 and normalizing each data point proportionately. Metabolite measurements are thus presented as normalized mass spectrometry-based intensities. ${ }^{*} p<0.05 ;{ }^{* *} p<0.01$; ${ }^{* * *} p<0.001$. PTECs, proximal tubular epithelial cells; CONT, control; DHT, dihydrotestosterone; EST, 17ß-estradiol; WT, wild-type; $\alpha-K G$, alpha-ketoglutarate; TCA, tricarboxylic acid; T2DM, type 2 diabetes mellitus. 
A

C

ロ MALE ロ FEMALE

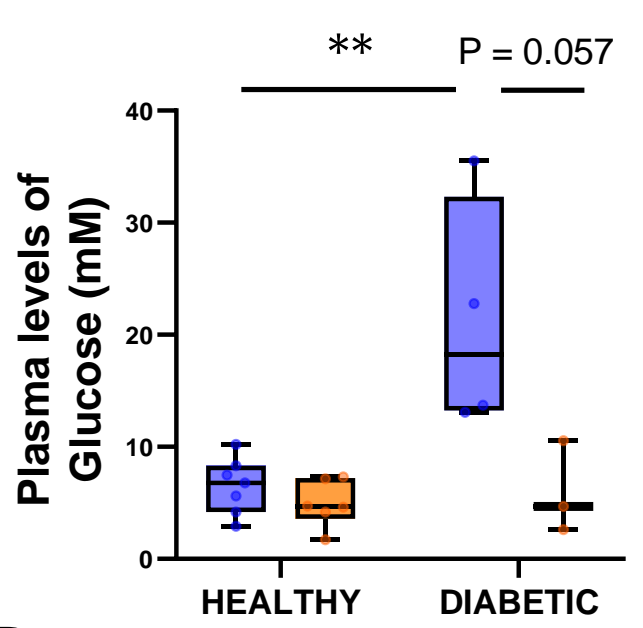

B

8 week-old healthy and diabetic mice

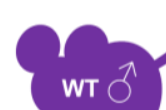

16 week-old healthy and diabetic mice

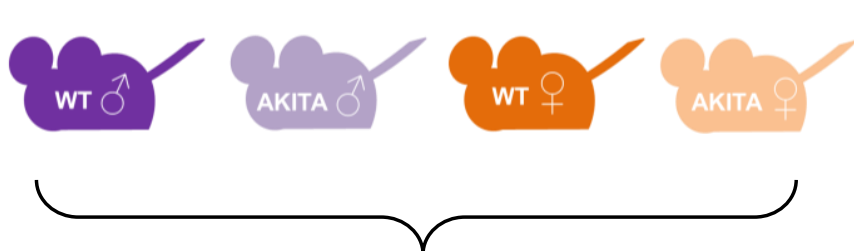

Plasma collection

$$
\begin{gathered}
\text { BioProfile FLEX2 } \\
\text { analyzer }
\end{gathered}
$$

Metabolite measurement

E

10-18 year-old subjects with and without T2DM (iCARE human cohort)

D

$\square$ MALE $\square$ FEMALE

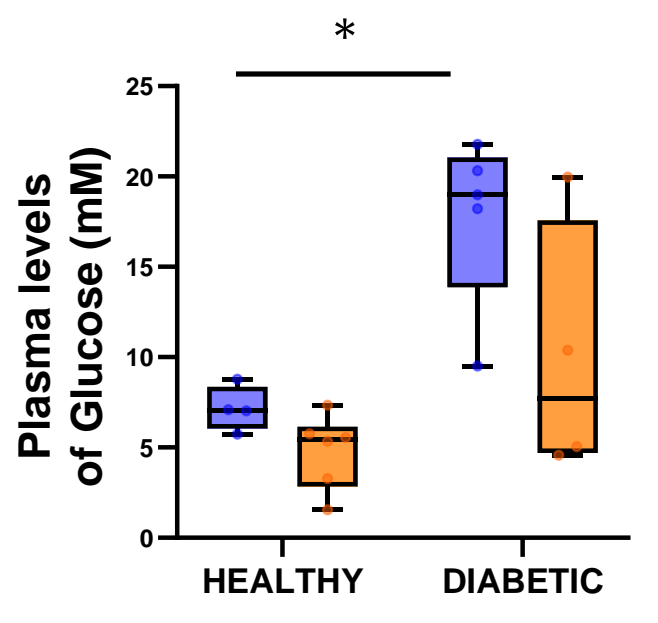

F $\square$ MALE $\square$ FEMALE
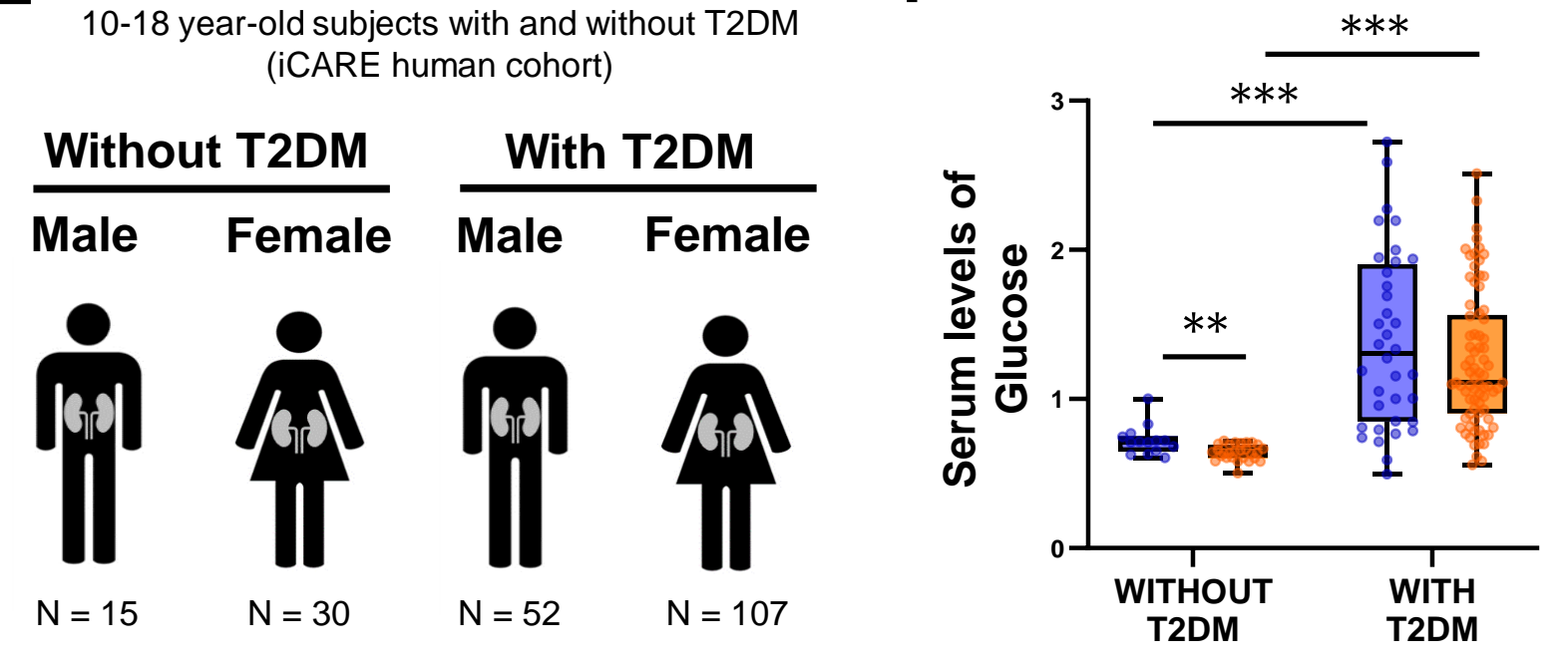
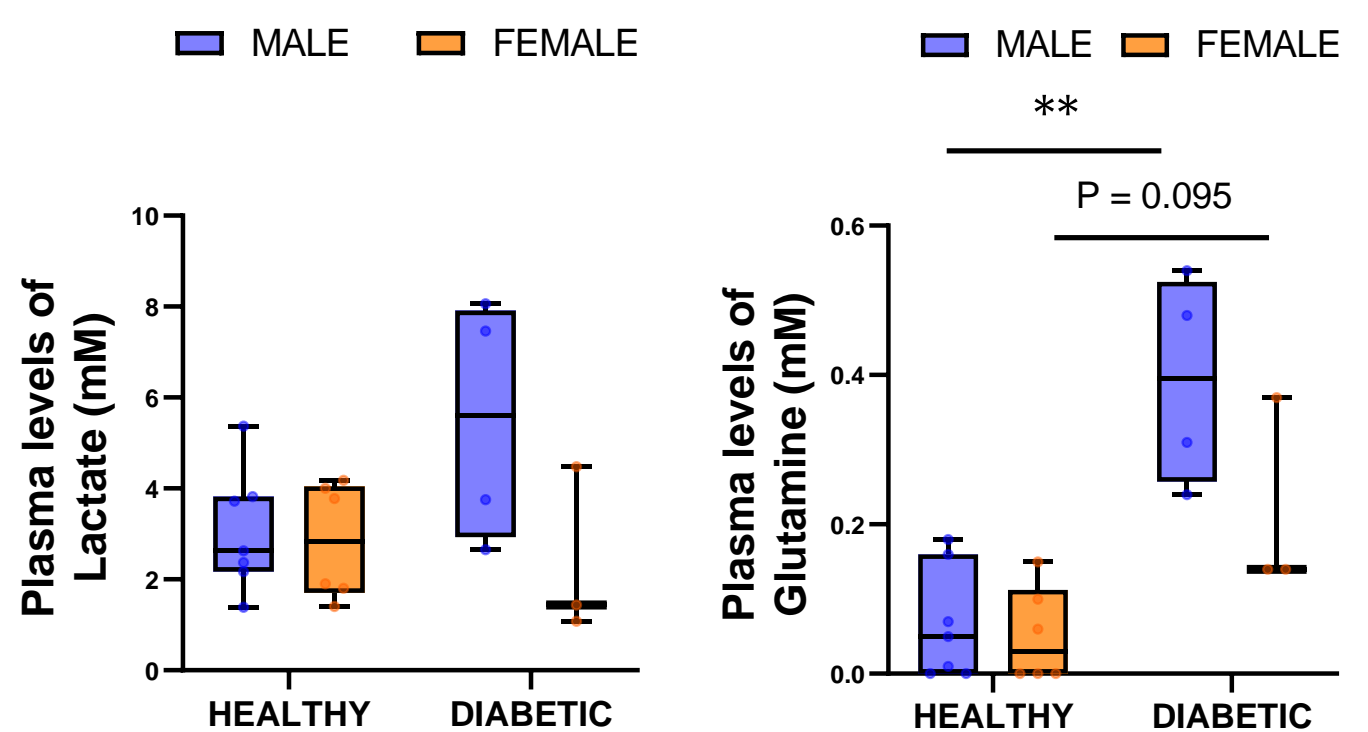

$\square$ MALE $\square$ FEMALE

$\square$ MALE $\square$ FEMALE
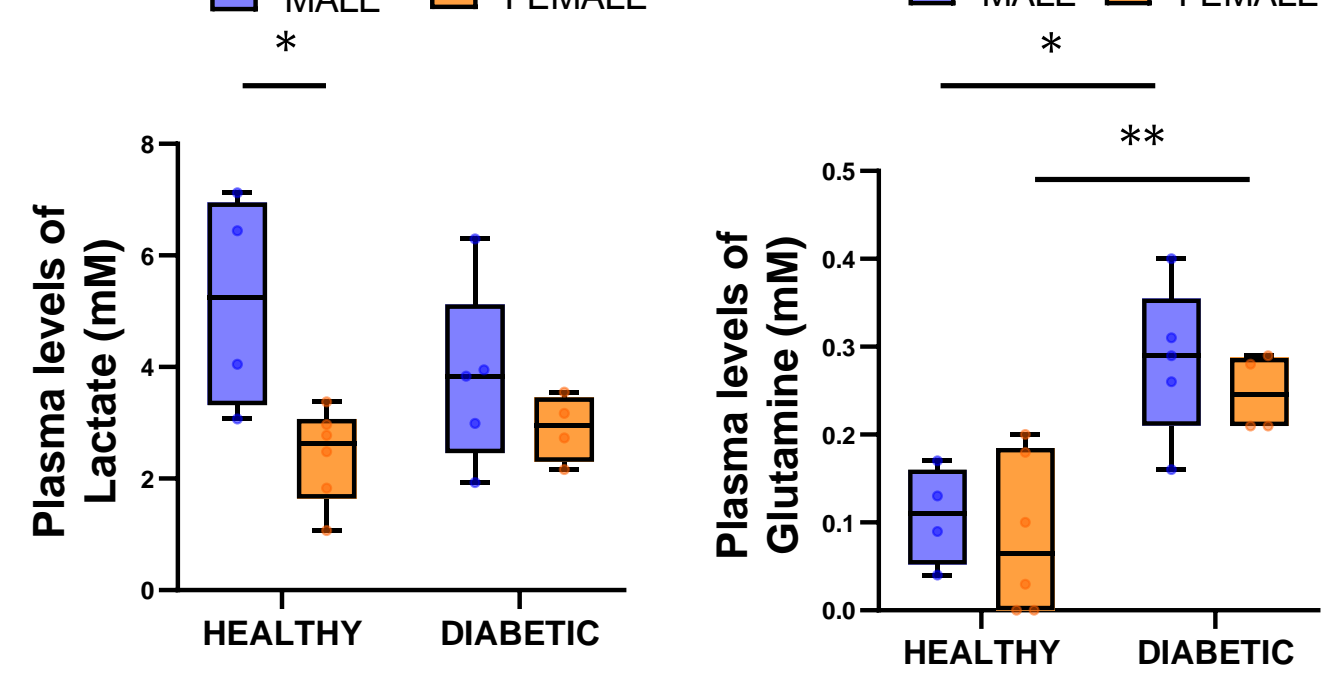

$\square$ MALE $\square$ FEMALE

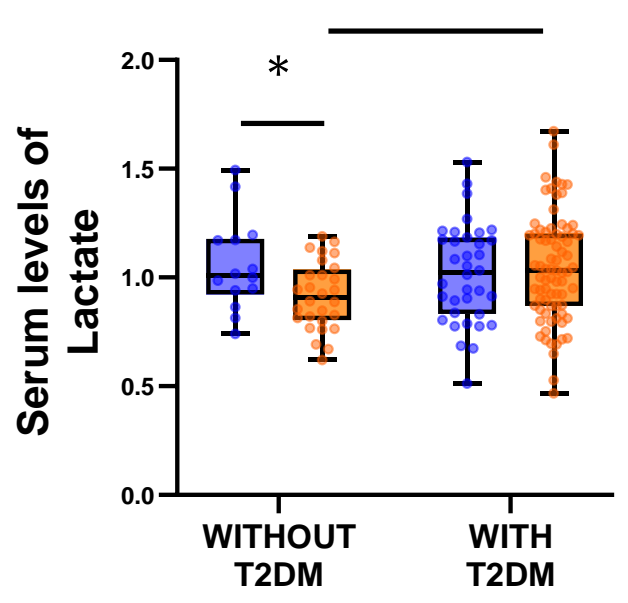

$\square$ MALE $\square$ FEMALE

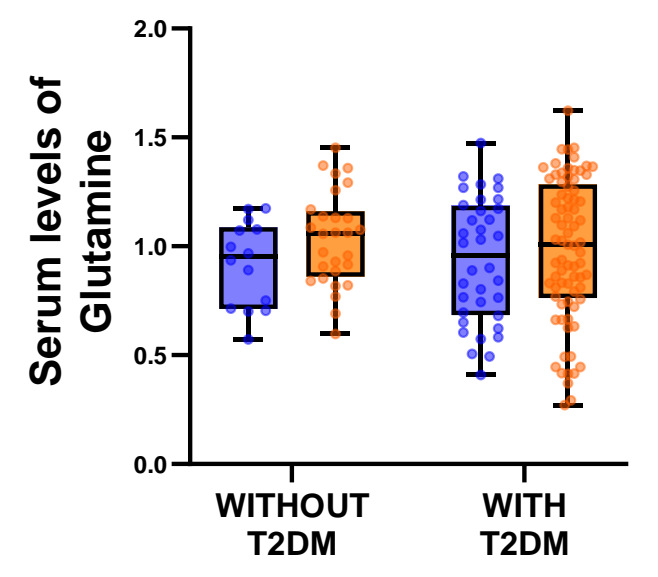

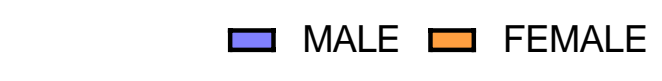

FIGURE 5

$\square$ MALE $\square$ FEMALE

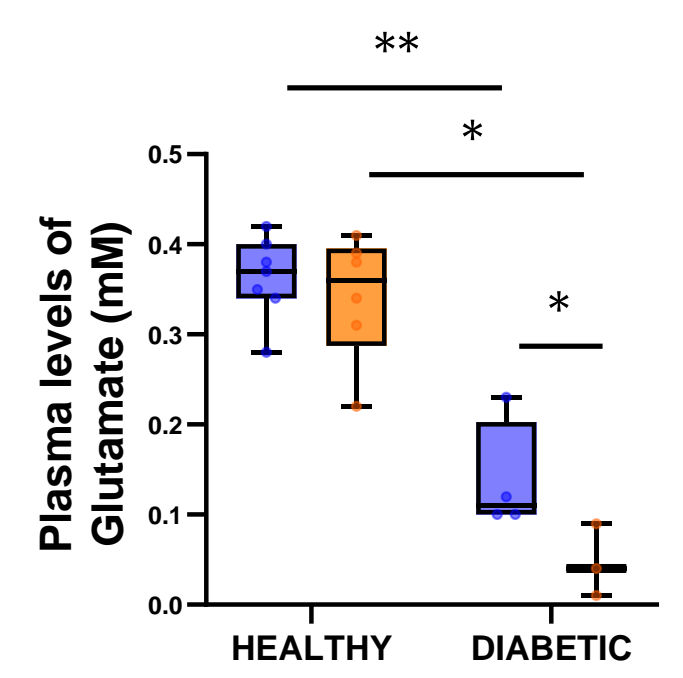

$\square$ MALE $\square$ FEMALE

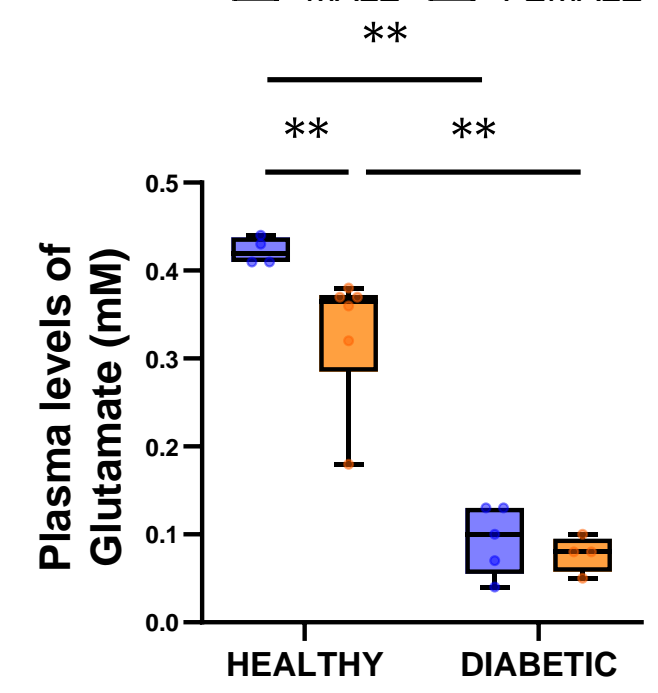

ロ MALE ロ FEMALE

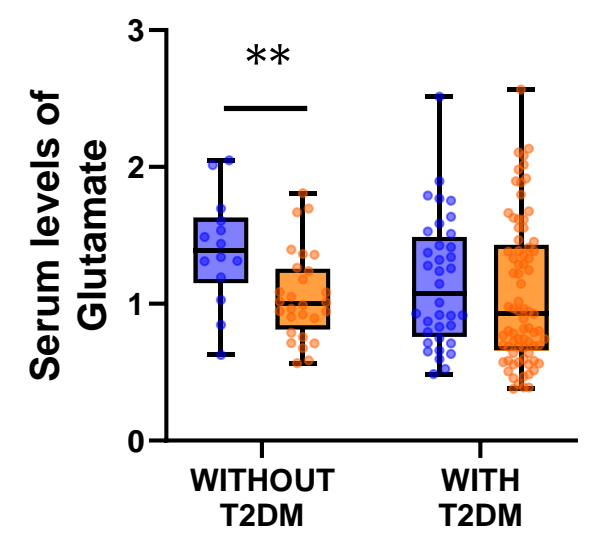


bioRxiv preprint doi: https://doi.org/10.1101/2021.08.23.457385; this version posted August 24, 2021. The copyright holder for this preprint (which was not certified by peer review) is the author/funder, who has granted bioRxiv a license to display the preprint in perpetuity. It is made

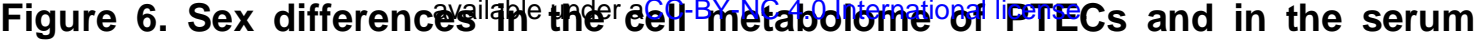

metabolome of healthy and diabetic individuals. Metabolome analysis was conducted in male and female PTECs exposed to normal glucose $(5.5 \mathrm{mM})$ for $16 \mathrm{~h}$ $(n=3 /$ sex; $n=6$ replicates/donor). The analysis revealed sex differences in the intracellular levels of key metabolites of the TCA cycle and the glutathione cycle. Differences in intracellular metabolite levels between the two groups were determined using a t-test adjusted for $5 \%$ false discovery rate. ${ }^{*} \mathrm{q}<0.05(\mathrm{~A})$. Sex differences in the levels of pyruvate, $\alpha-K G$, succinate, fumarate, and malate were examined in the serum of men without T2DM ( $n=15)$, women without T2DM ( $n=27)$, men with T2DM $(n=47)$, and women with T2DM ( $\mathrm{n}=91)$. Data normalization was performed to correct variation emerging from inter-day tuning differences on instrument performance. Each compound was corrected in run-day blocks by registering the medians to 1.00 and normalizing each data point proportionately. Metabolite measurements are thus presented as normalized mass spectrometry-based intensities (B). Male sex was independently associated with increased circulating levels of $9 \gamma$-glutamyl-aminoacids. The levels of 6 of these $\gamma$ glutamyl-aminoacids are shown in panel C. Among these individuals, the urinary excretion of glycine, isoleucine, methionine, valine, serine, and threonine was altered by sex and/or diabetes (D). Panels $B-E:{ }^{*} p<0.05$ and $q<0.1 ;{ }^{* *} p<0.01$ and $q<0.1 ;{ }^{* * *} p<0.001$ and $\mathrm{q}<0.1$. TCA, tricarboxylic acid; $\alpha-K G$, alpha-ketoglutarate; $\mathrm{GSH}$, reduced glutathione; GSSG, oxidized glutathione; T2DM, type 2 diabetes mellitus. The metabolic cycles in panel A were created with BioRender.com. 







\section{Figure 7. Identification of KDM6A as a regulator of the metabolic function of female PTECs.}

Kidney genes significantly altered by sex (Nephroseq database), and proteins altered by sex hormones were analyzed using CATRIN transcriptional regulator (TR) database. The analysis revealed a significant enrichment $(p<0.05)$ of 129 TRs among EST-regulated proteins. In turn, 818 TRs were significantly enriched $(q<0.05)$ among genes upregulated in the human female kidney (A). The Venn diagram illustrates the overlap between TRs significantly enriched among kidney molecular signatures linked to male sex, female sex, DHT, and EST. Key TRs linked to female sex are highlighted in orange, while TRs linked to EST are highlighted in green (B). Key TRs and targets emerging from the analysis and relevant to female sex are illustrated (C). The color of each box indicates that the TR/target was enriched/increased by female sex (orange) and/or by EST (green). KDM6A gene expression was determined in male and female PTECs exposed to vehicle (CONT), 100nM DHT, or 100nM EST for 16h, and normalized to RPL31 ( $n=3 /$ sex; $n=4-6 /$ treatment) (D). Kidney Kdm6a gene expression was assessed in 19-week-old male and female mice subjected to sham-operation, gonadectomy (GDX) or ovariectomy (OVX) ( $n=5-10$ animals/group) (E). Male and female PTECs were treated with vehicle (DMSO), $2.5 \mu M$ of GSK-J4 (KDM6A inhibitor), or $2.5 \mu \mathrm{M}$ of GSK-J5 (inactive analog) for $48 \mathrm{~h}$ ( $\mathrm{n}=3 / \mathrm{sex}$; $\mathrm{n}=4$ 8/treatment). After treatment, OCR and glycolysis (ECAR) were measured in a Seahorse XFe96 analyzer (F-G). To induce metabolic stress, the following sequence of drugs was injected: $1 \mu \mathrm{M}$

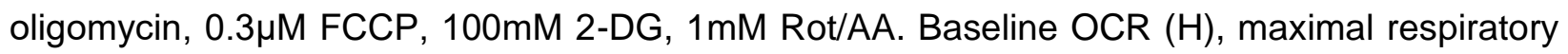
capacity $(\mathrm{I})$, reserve respiratory capacity $(\mathrm{J})$, and ATP-linked respiration $(\mathrm{K})$, were calculated from the OCR curves in panel F. In turn, basal glycolysis (L) and maximal glycolytic capacity (M) were calculated from the ECAR curve in panel G. Cell viability was assessed by measuring endogenous formazan formation (MTT assay) (N), uptake of cytocalcein (viability dye) (O), and intracellular ATP levels (P). Intracellular superoxide ion levels (Q) and release of lactate dehydrogenase $(R)$ were also assessed. In panels F-G, a t test was used at each time point to assess statistical significance between the two groups In panels D-E and H-R, Group-to-group 
differences were determined using pairwise t tests for variables following a normal distribution, and Mann-Whitney tests for variables with a non-parametric distribution. ${ }^{*} p<0.05 ;{ }^{* *} p<0.01$. PTECs, proximal tubular epithelial cells; CONT, control; DHT, dihydrotestosterone; EST, estradiol; AUC, area under the curve; ECAR, extracellular acidification rate; OCR, oxygen consumption rate; FCCP, p-trifluoromethoxy carbonyl cyanide phenyl hydrazone; 2-DG, 2deoxyglucose; Rot, rotenone; $\mathrm{AA}$ : antimycin $\mathrm{A}$; $\mathrm{DMSO}$, dimethyl sulfoxide; RFU, relative fluorescence units. The illustration in panel C was created with BioRender.com. 
A

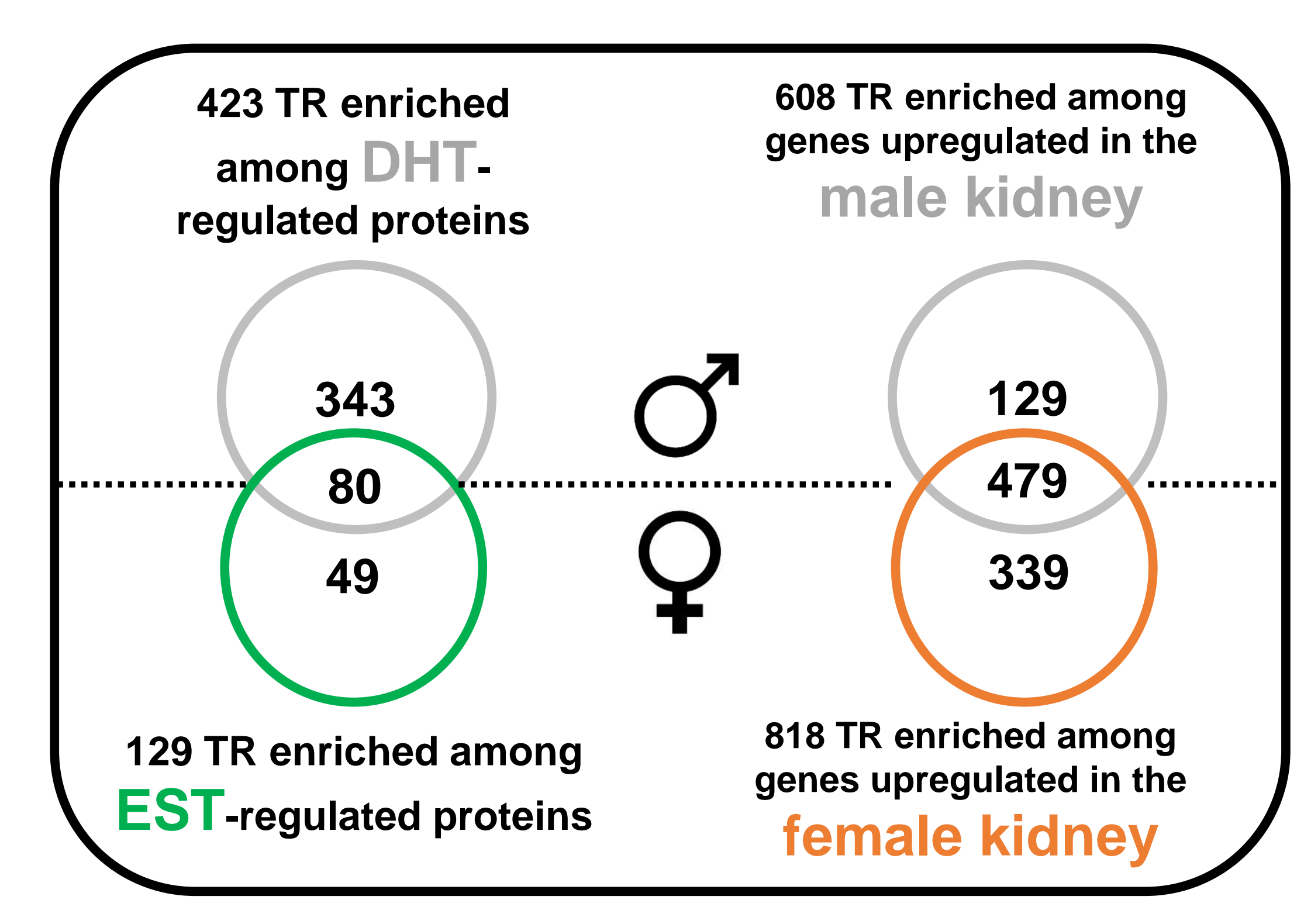

B

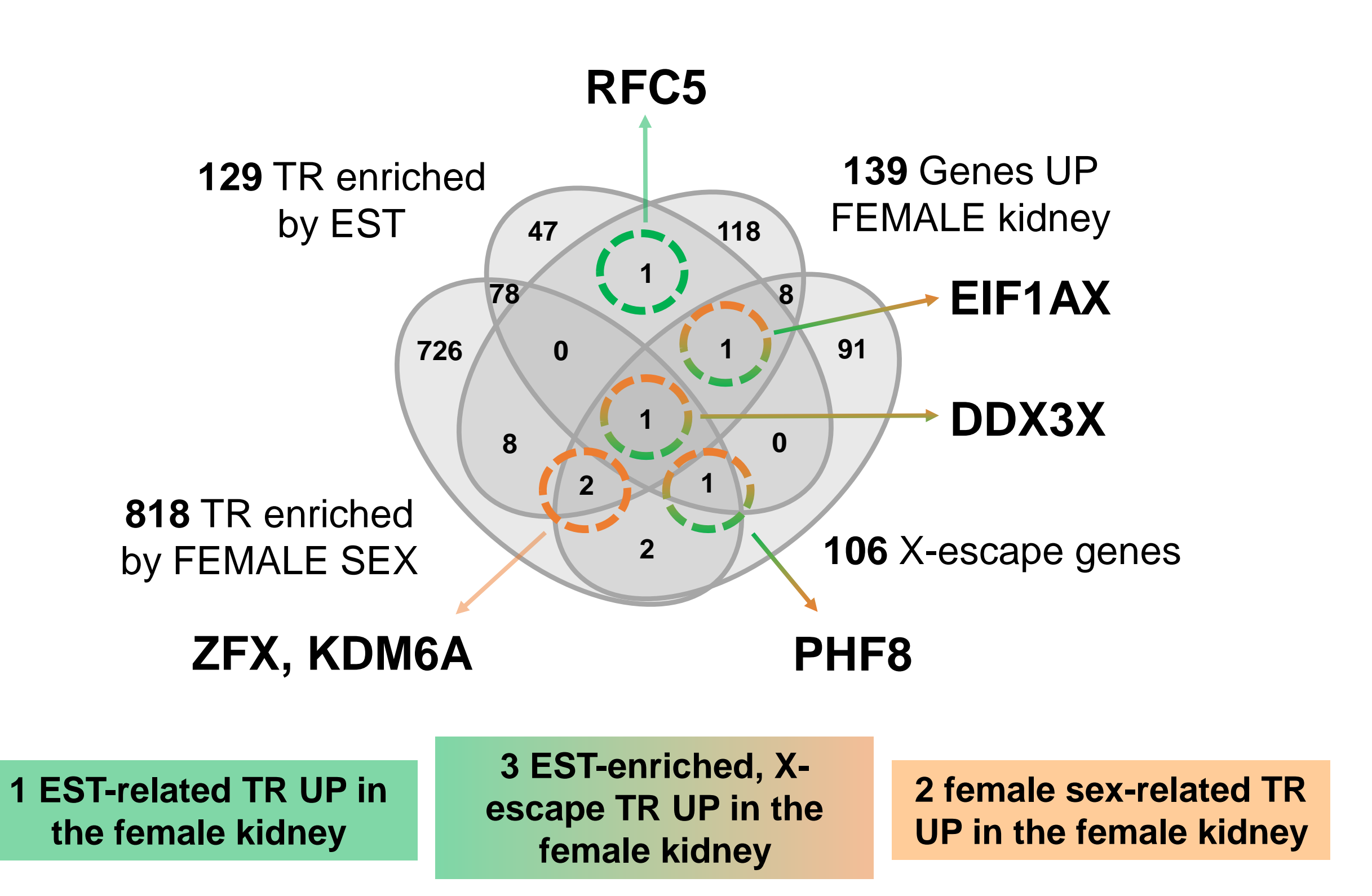

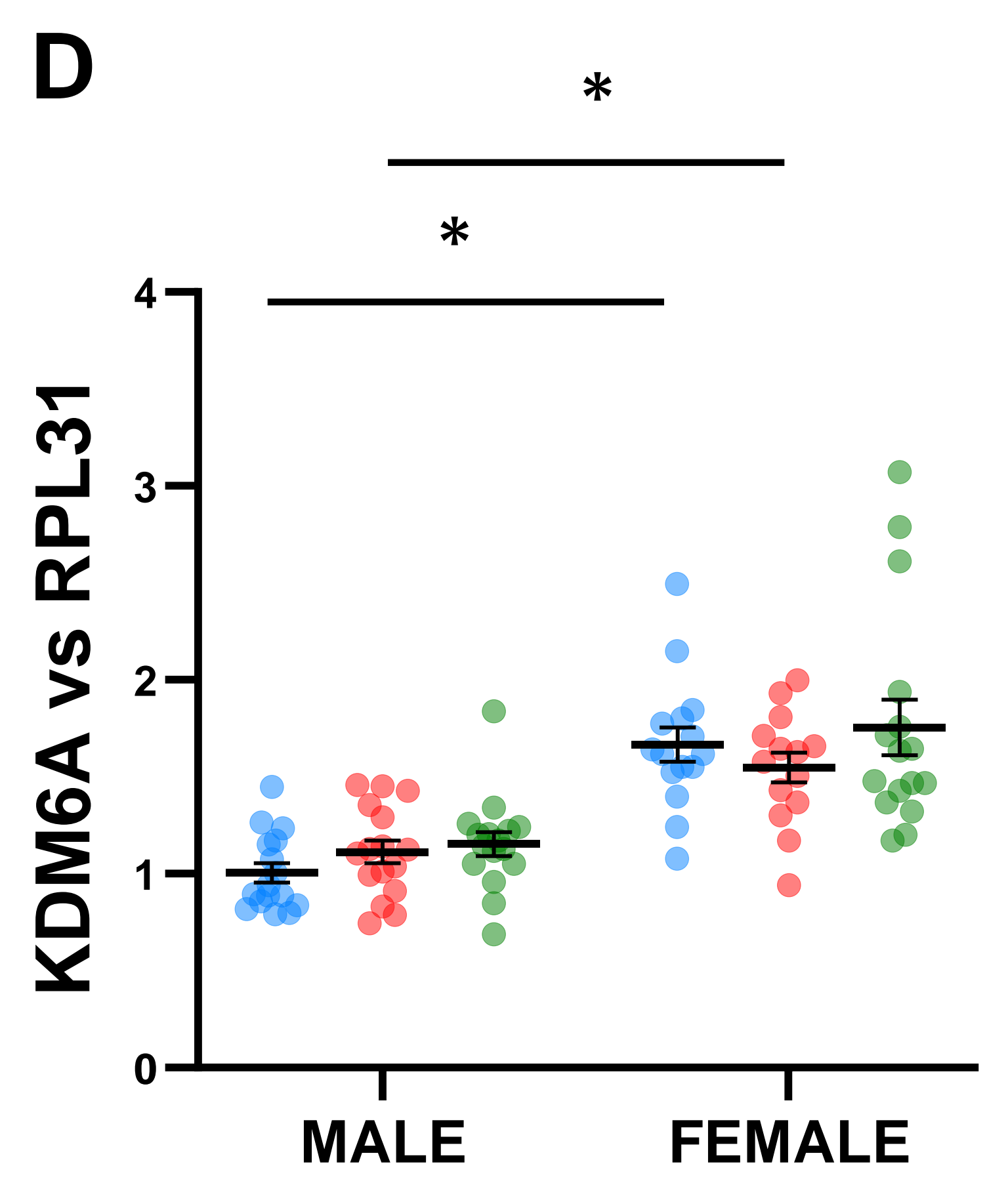

E
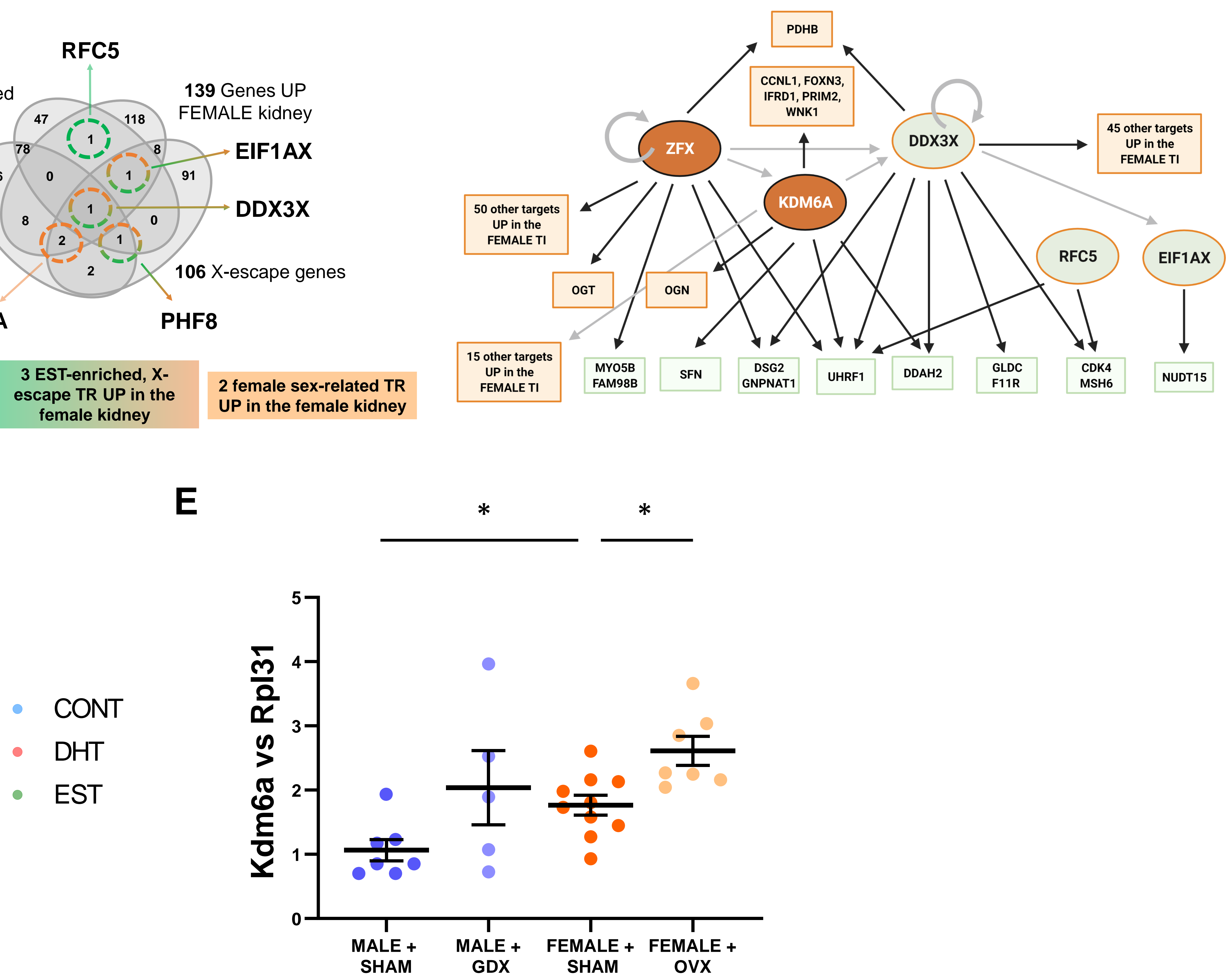

$\mathbf{F}$

Treatment: Vehicle (0.1\% DMSO), $2.5 \mu \mathrm{M}$ of GSK-J4 or $2.5 \mu \mathrm{M}$ GSK-J5 for $48 \mathrm{~h}$ MALE PTECS

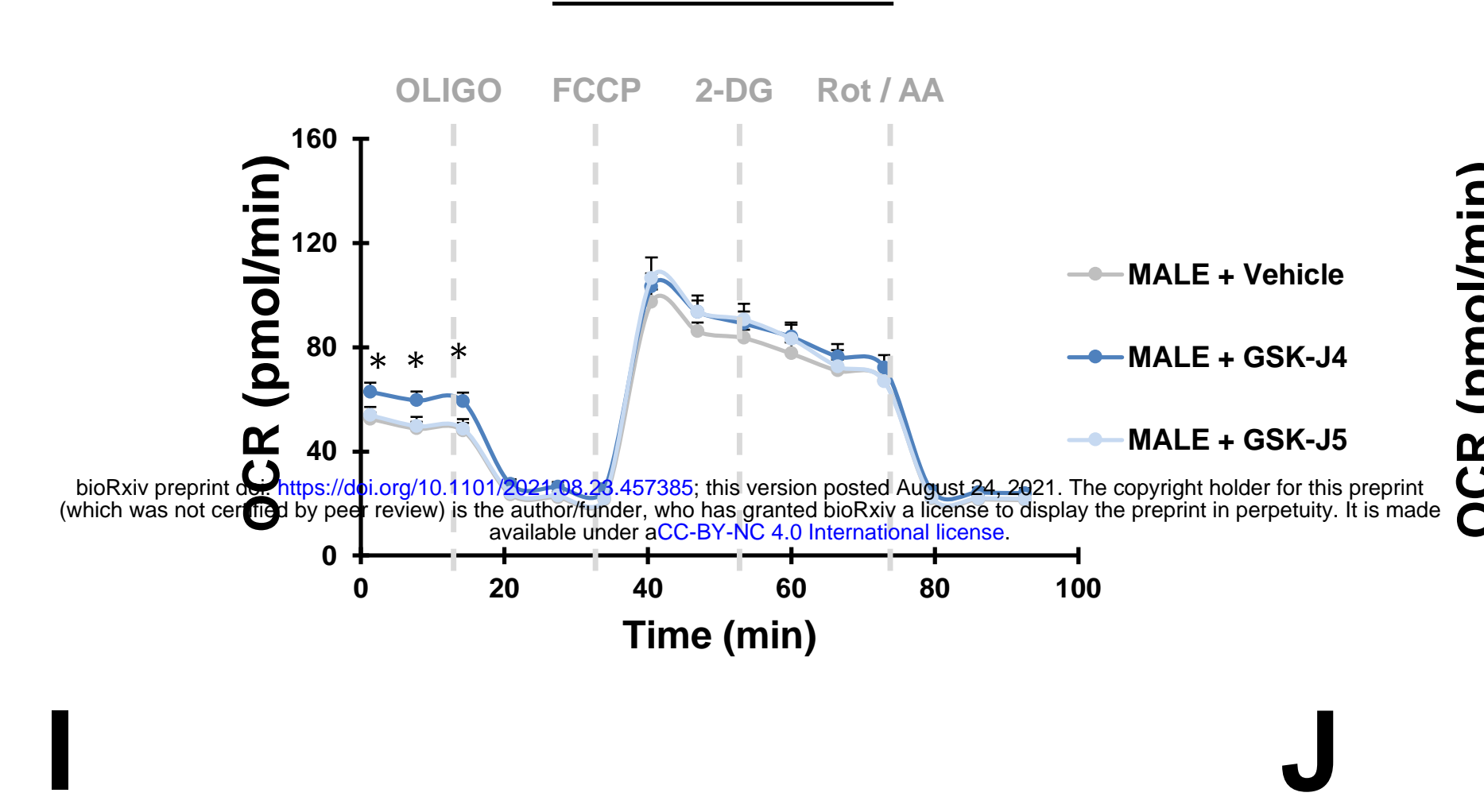

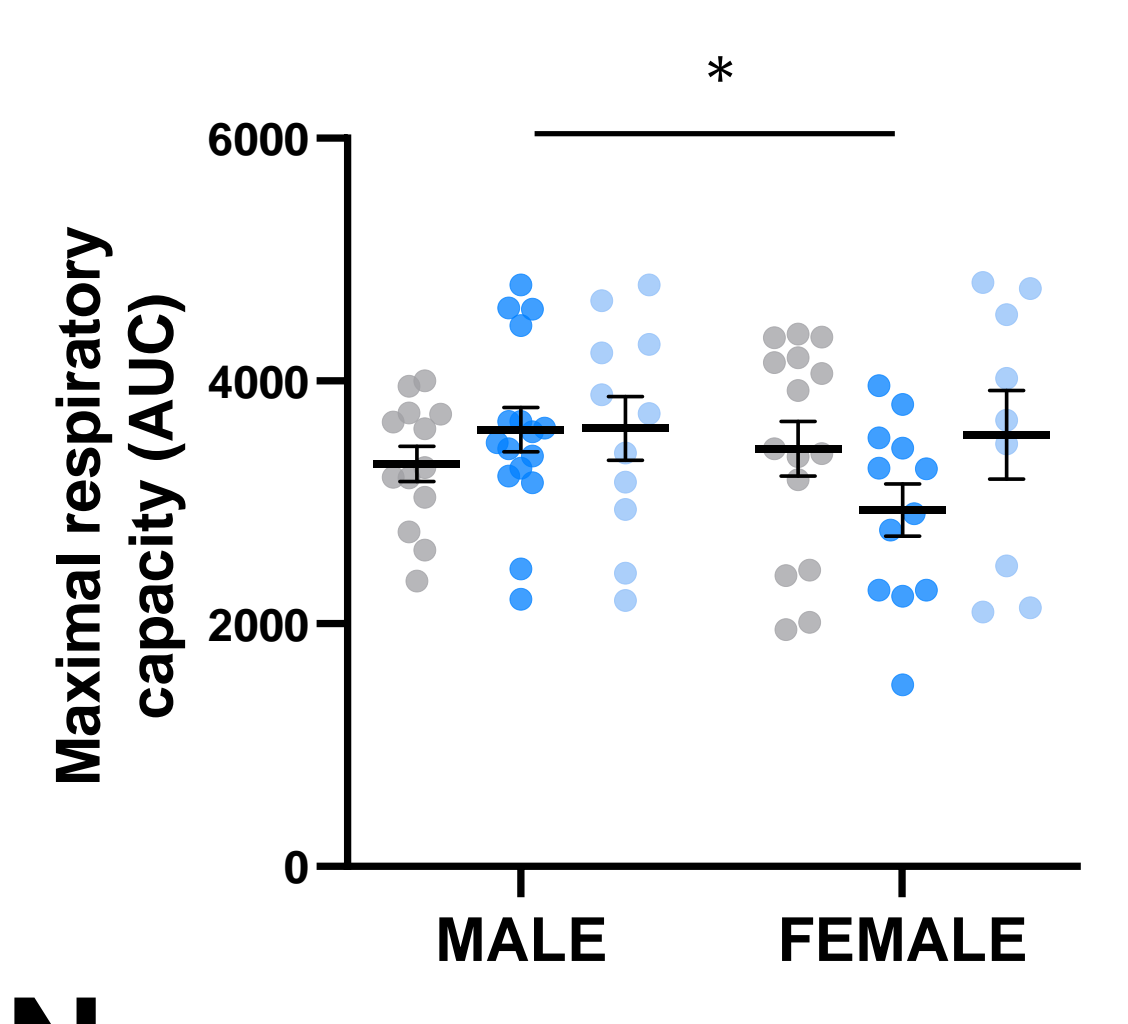

$\mathbf{N}$

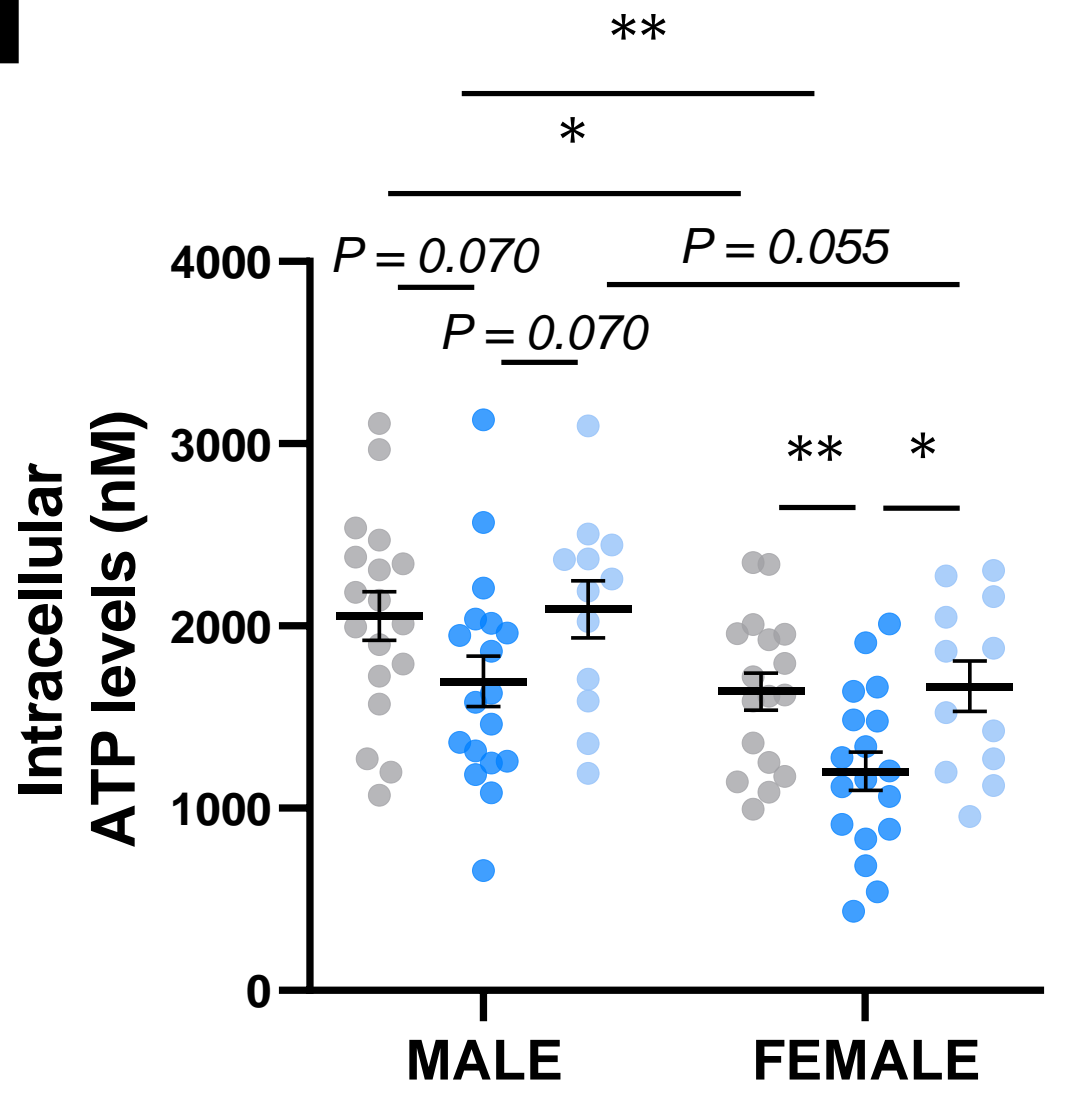

FEMALE PTECS
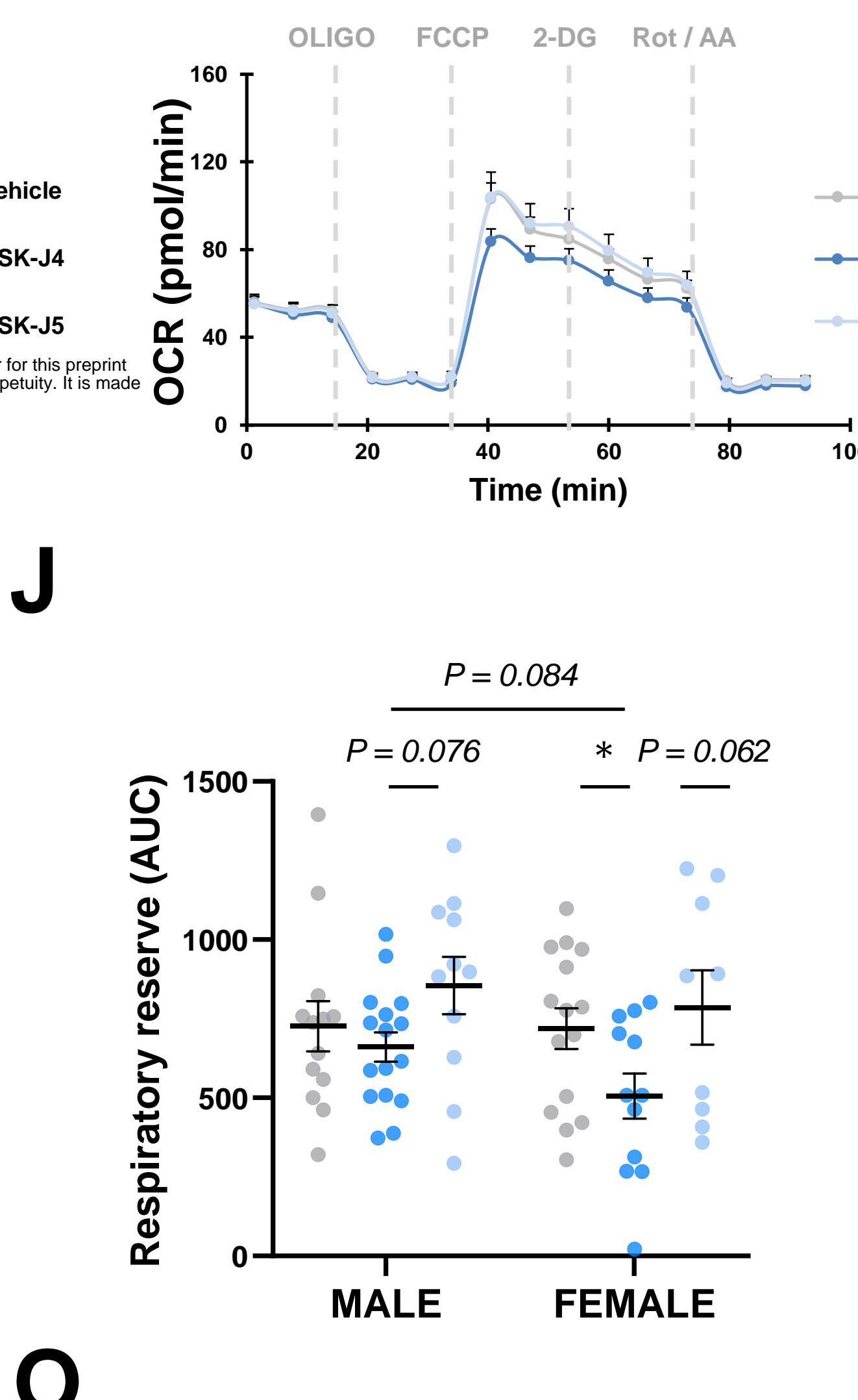

0

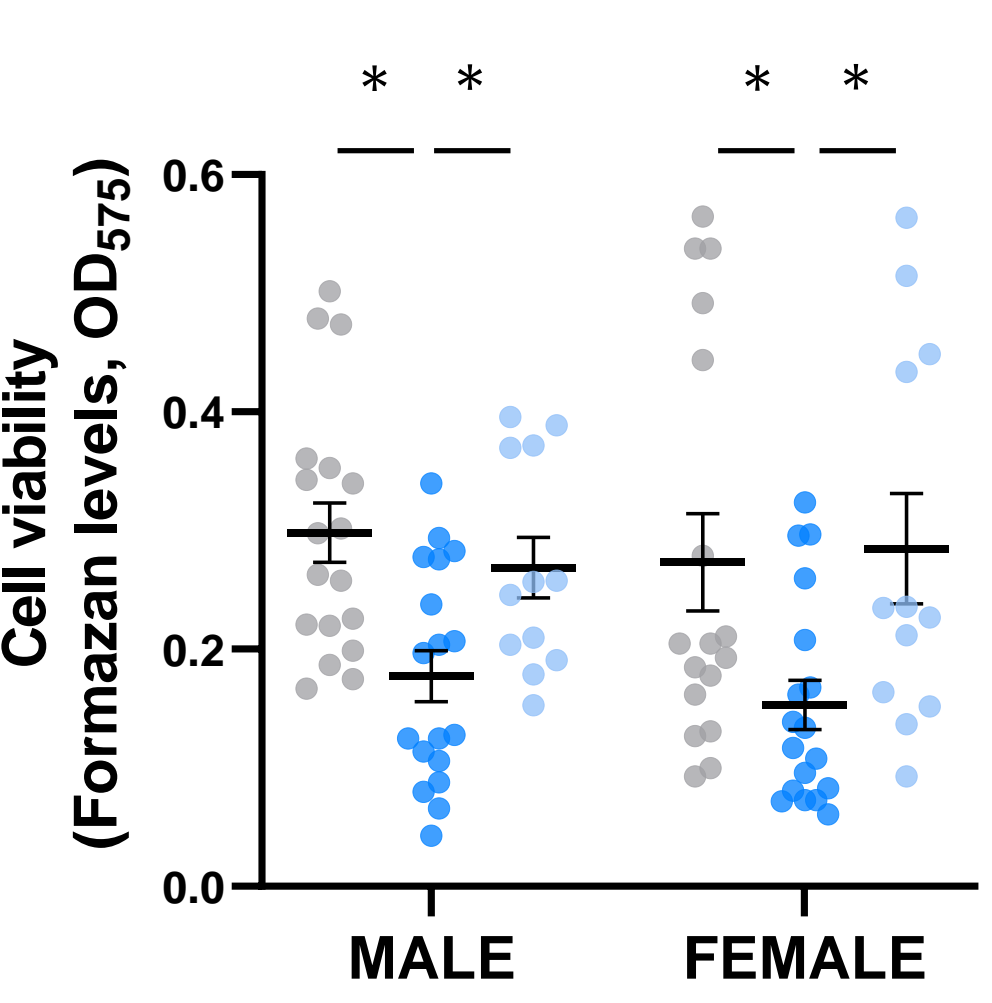

G

Treatment: Vehicle $(0.1 \%$ DMSO), $2.5 \mu \mathrm{M}$ of GSK-J4 or $2.5 \mu \mathrm{M}$ GSK-J5 for $48 \mathrm{~h}$ MALE PTECs

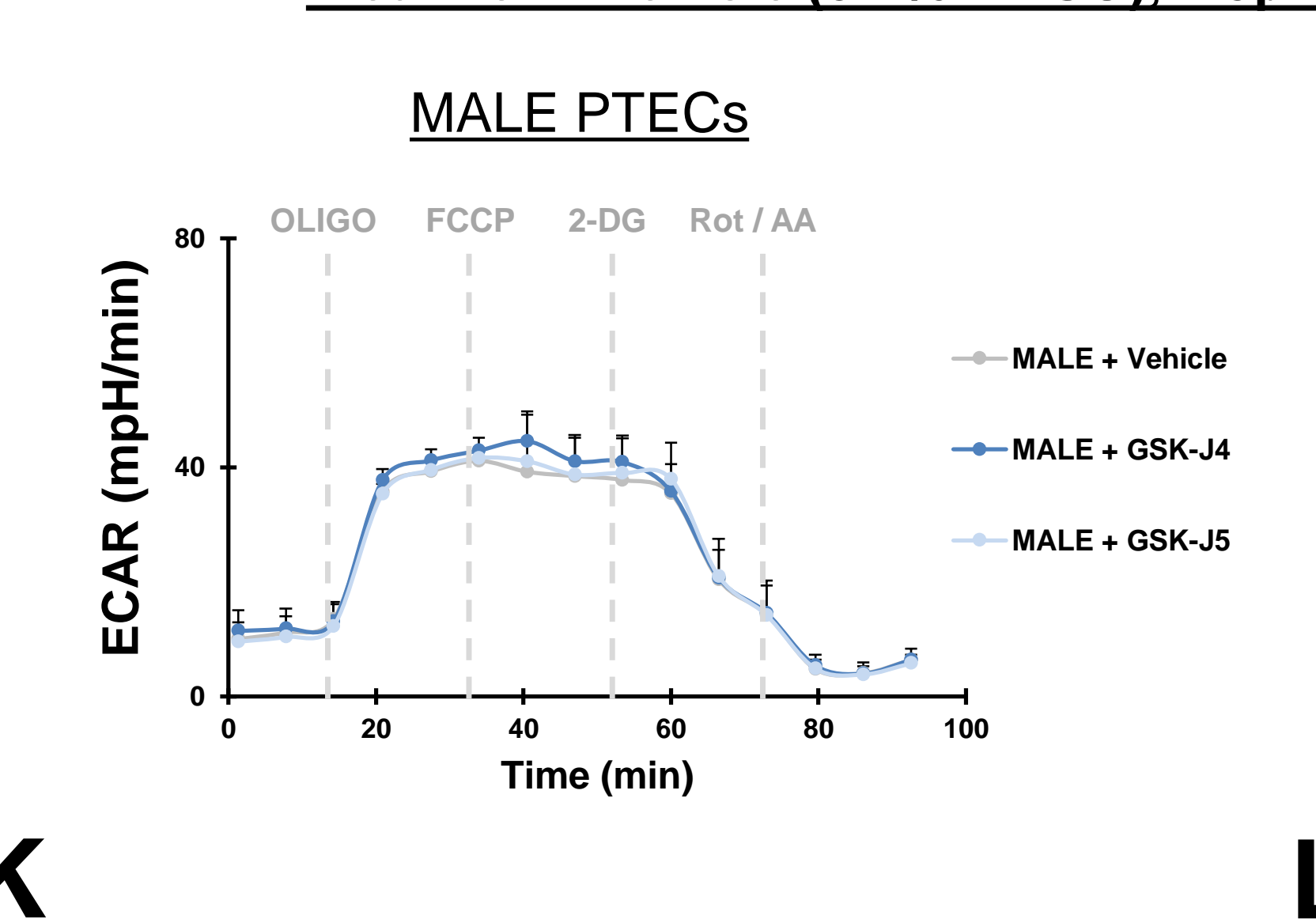

K

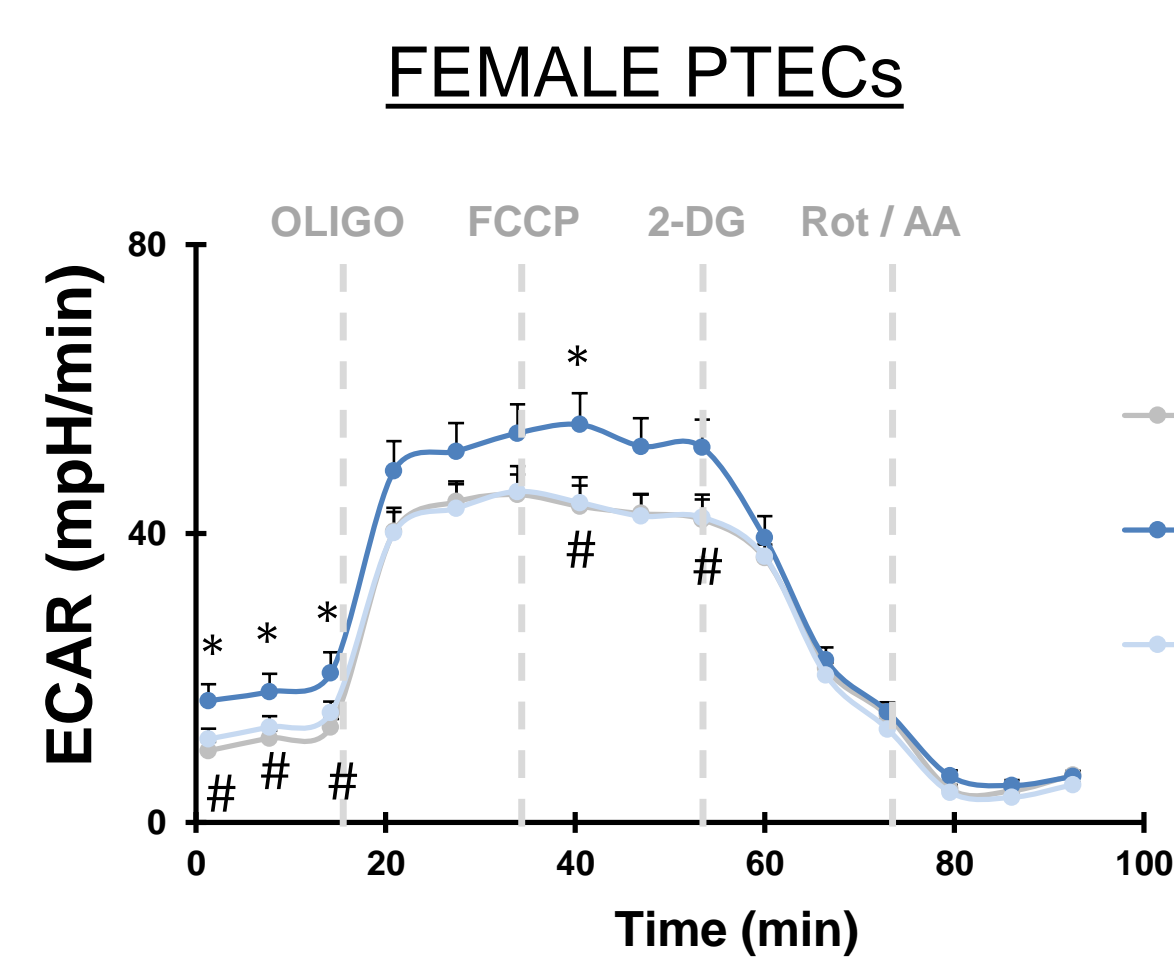

L

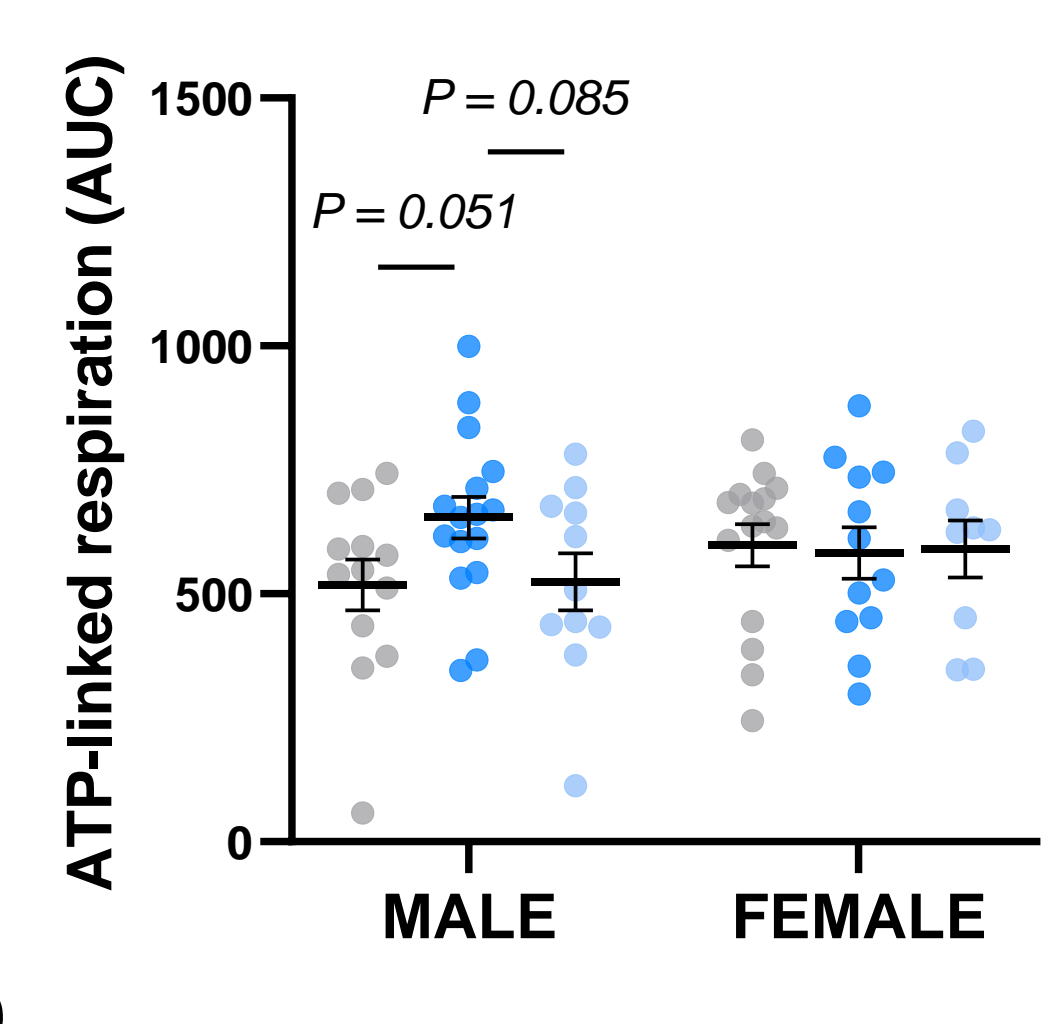

$\mathbf{P}$

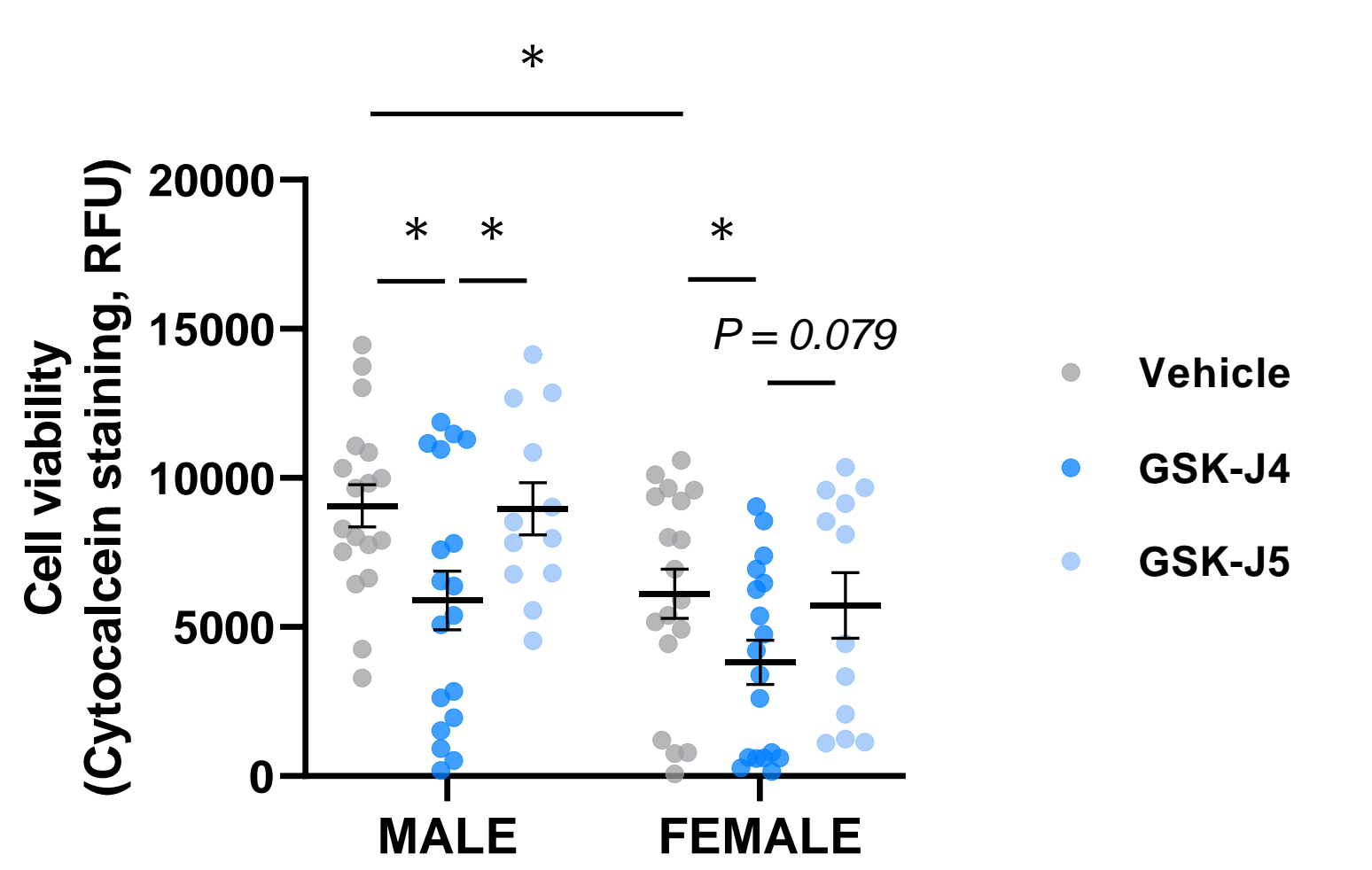

H

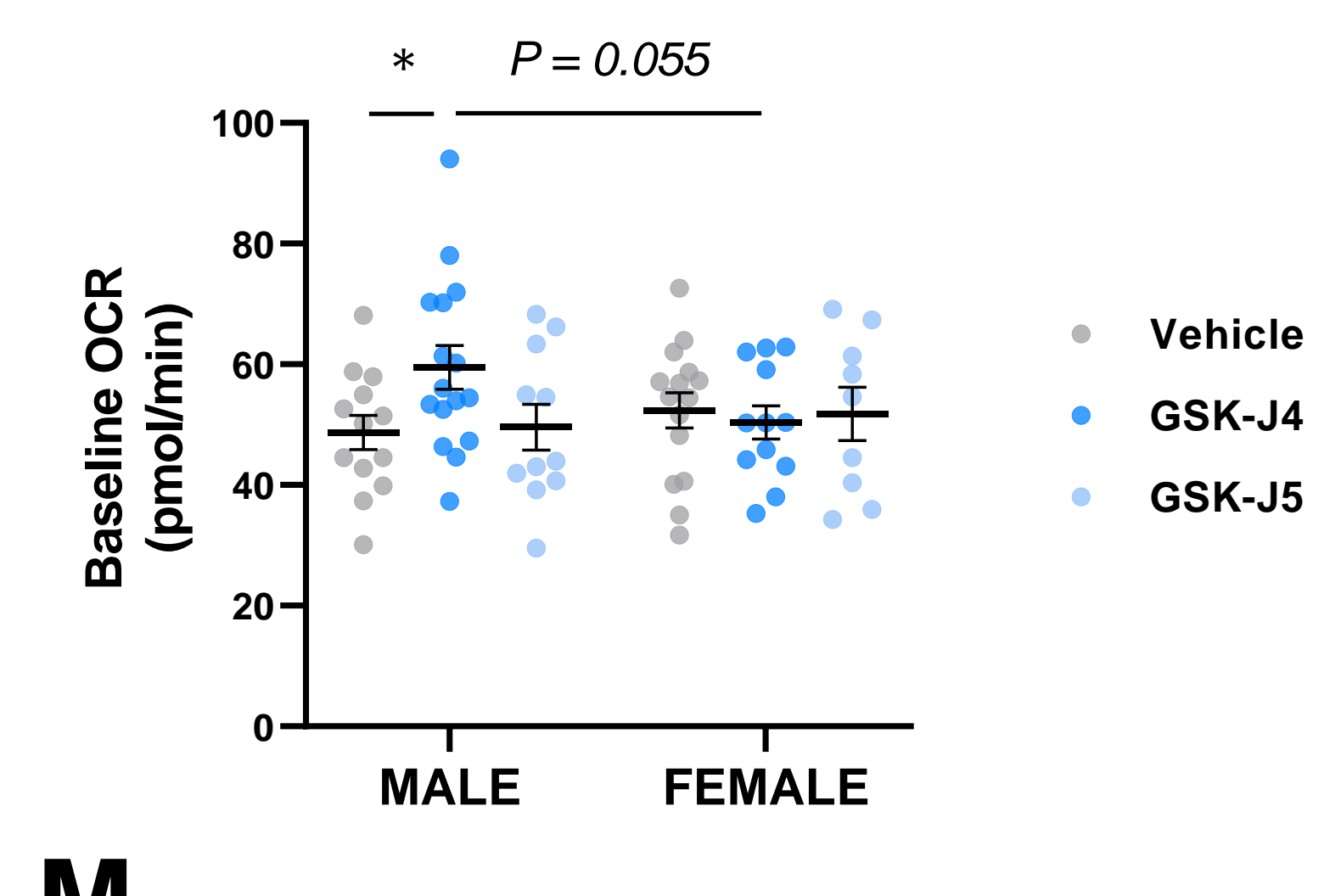

M

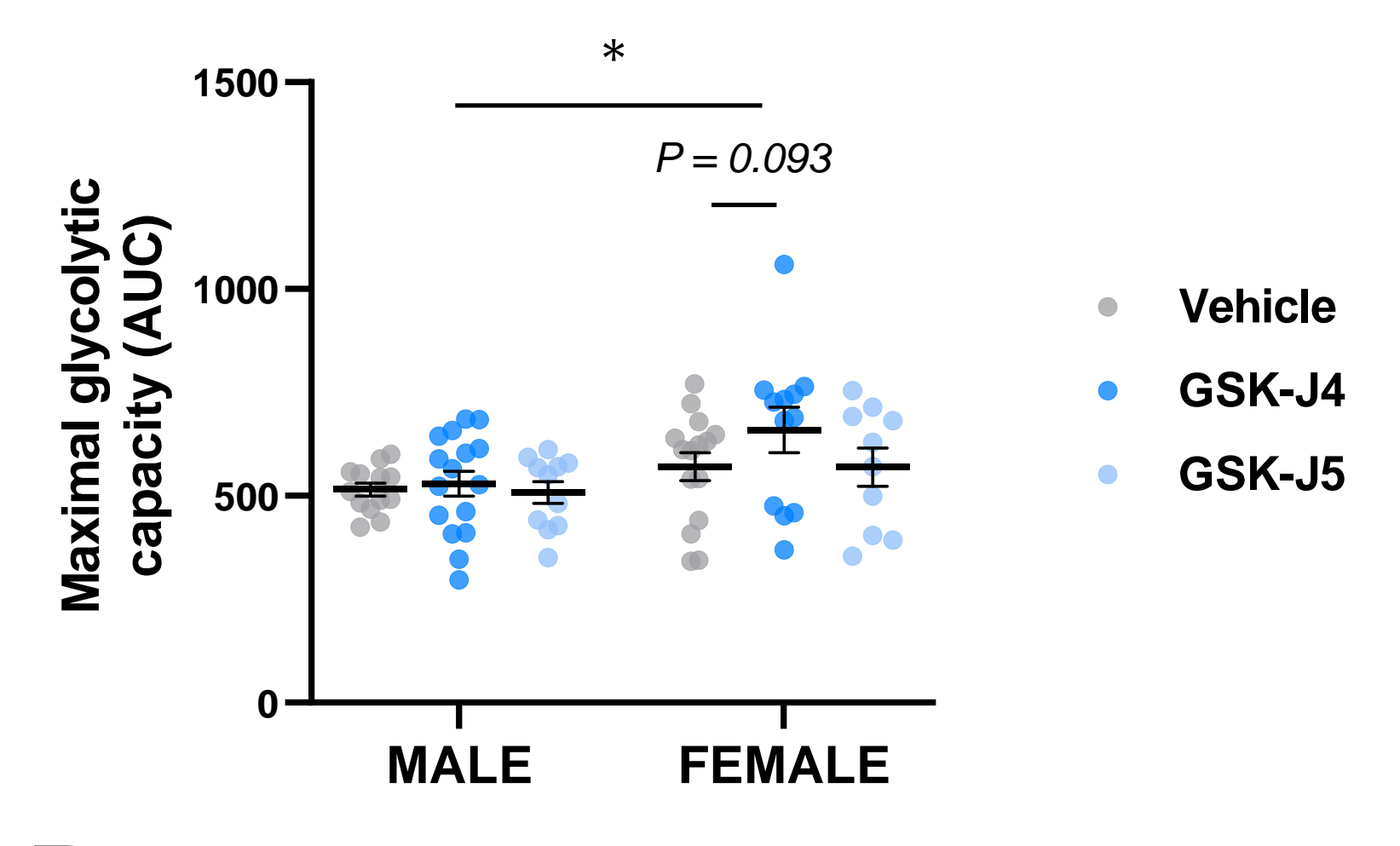

R

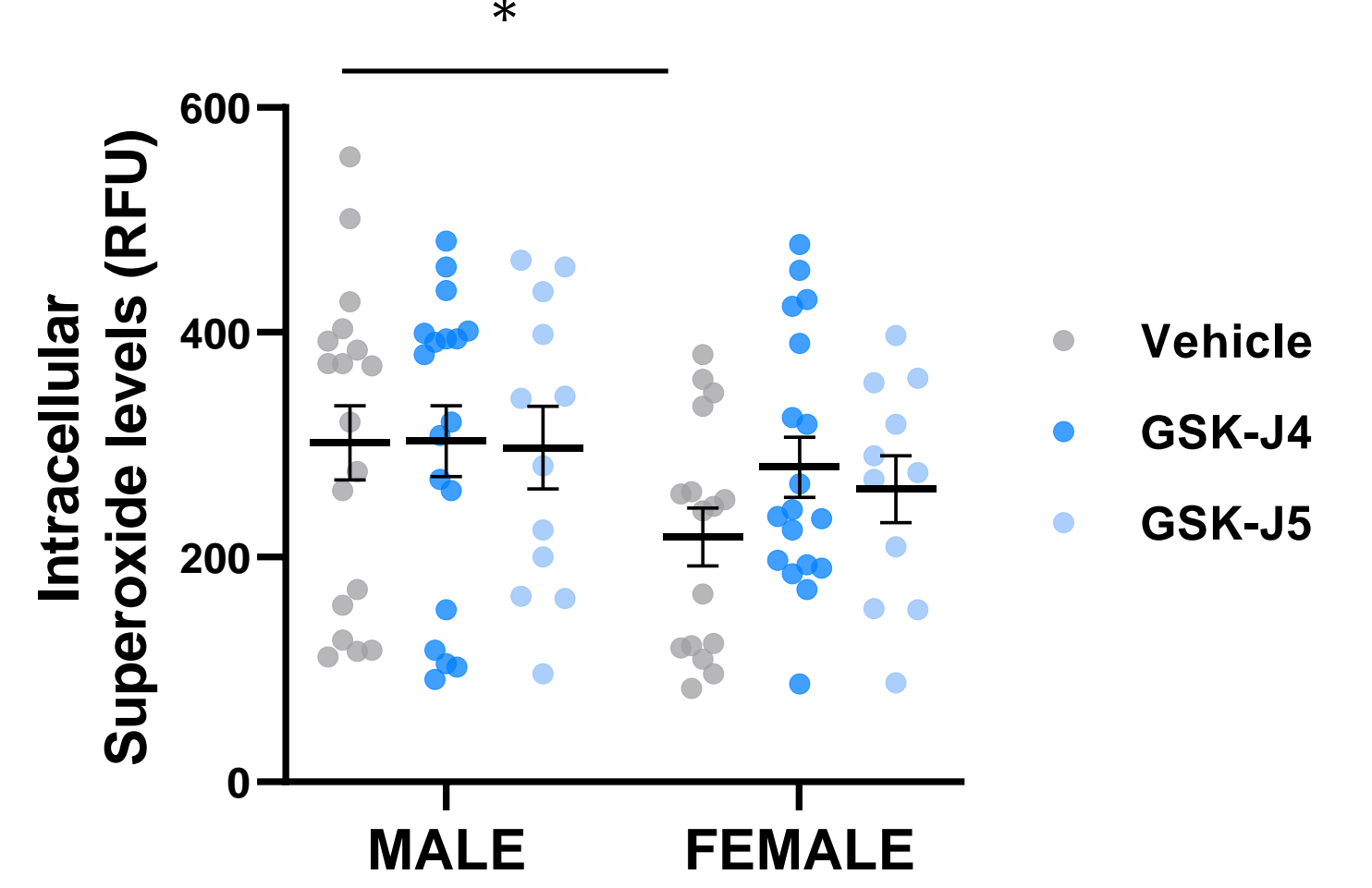

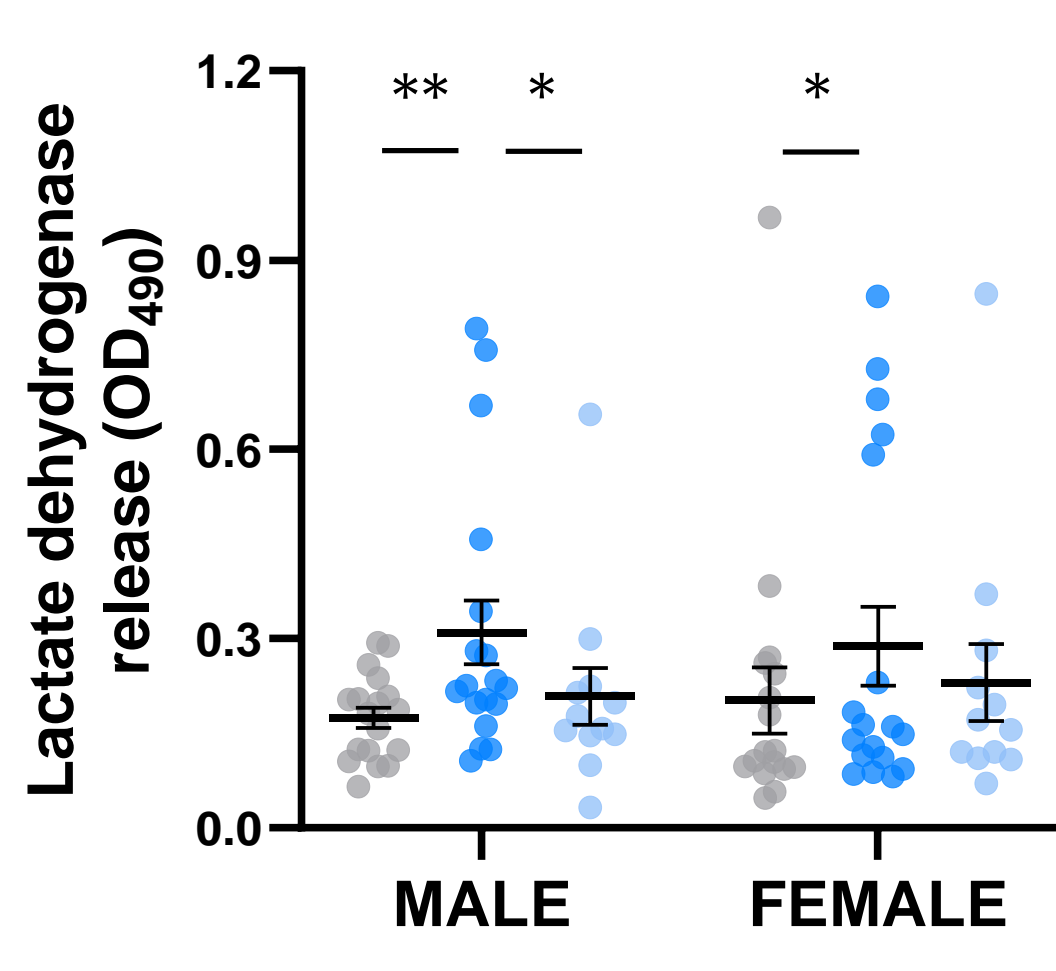


bioRxiv preprint doi: https://doi.org/10.1101/2021.08.23.457385; this version posted August 24, 2021. The copyright holder for this preprint (which was not certified by peer review) is the author/funder, who has granted bioRxiv a license to display the preprint in perpetuity. It is made

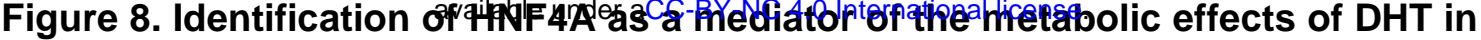

male PTECs. To identify transcriptional regulators (TRs) responsible for the observed metabolic sex differences, kidney genes significantly altered by sex (Nephroseq database) and proteins altered by sex hormones ${ }^{31}$ were analyzed using CATRIN TR database. The analysis revealed a significant enrichment $(p<0.05)$ of 423 TRs among DHT-regulated proteins. In turn, 608 TRs were significantly enriched $(q<0.05)$ among genes upregulated in the human male kidney $(\mathrm{A})$. The Venn diagram illustrates the overlap between TRs significantly enriched among kidney molecular signatures linked to male sex, female sex, DHT, and EST. Key TRs linked to male sex are highlighted in purple, while TRs of interest linked to DHT are highlighted in red (B). Relevant TRs and targets emerging from the analysis and relevant to male sex are illustrated. The color of each box indicates that the TR/target was enriched/increased by male sex (purple) and/or by DHT (red). HNF4A gene expression was determined in male and female PTECs exposed to vehicle (CONT), 100nM DHT, or 100nM EST for 16h, and normalized to RPL31 ( $n=3 /$ sex; $n=4-6 /$ treatment) (D). Kidney Hnf4a gene expression was assessed in 19-week-old male and female mice subjected to sham-operation, gonadectomy (GDX) or ovariectomy (OVX) ( $n=5-10$ animals/group) (E). Male and female PTECs were treated for $16 \mathrm{~h}$ with vehicle or DHT in the presence or absence of DMSO or $0.1 \mu \mathrm{M} \mathrm{BI6015}$ (HNF4A inhibitor) ( $n=3 /$ sex; $n=3-4 /$ treatment). After the treatment, OCR and glycolysis (ECAR) were measured in a Seahorse XFe96 analyzer (F-G). To induce metabolic stress, the following sequence of drugs was injected: $1 \mu \mathrm{M}$ oligomycin, $0.3 \mu \mathrm{M} F C C P$, 100mM 2-DG, 1mM Rot/AA. Baseline OCR $(H)$, maximal respiratory capacity (I), reserve respiratory capacity $(\mathrm{J})$, and ATP-linked respiration $(\mathrm{K})$, were calculated from the OCR curves in panel F. In turn, basal glycolysis (L), maximal glycolytic capacity (M), and glycolytic reserve $(\mathrm{N})$ were calculated from the ECAR curve in panel $\mathrm{G}$. Surface levels of phosphatidylserine (PS) were measured as a marker of early apoptosis (O). Intracellular ATP levels were also measured (P). Panels F-G: * $\mathrm{p}<0.05$ vs CONT + Vehicle; \#p<0.05 vs DHT + Vehicle. Panels D-E, H-P: ${ }^{*} p<0.05 ;{ }^{* *} p<0.01$. PTECs, 
bioRxiv preprint doi: https://doi.org/10.1101/2021.08.23.457385; this version posted August 24, 2021. The copyright holder for this preprint (which was not certified by peer review) is the author/funder, who has granted bioRxiv a license to display the preprint in perpetuity. It is made

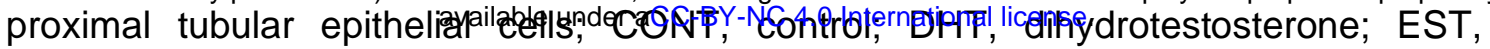

estradiol; AUC, area under the curve; ECAR, extracellular acidification rate; OCR, oxygen consumption rate; FCCP, p-trifluoromethoxy carbonyl cyanide phenyl hydrazone; 2-DG, 2-deoxyglucose; Rot, rotenone; AA: antimycin A; RFU, relative fluorescence units. The diagram in panel $\mathrm{C}$ was created with BioRender.com. 
A
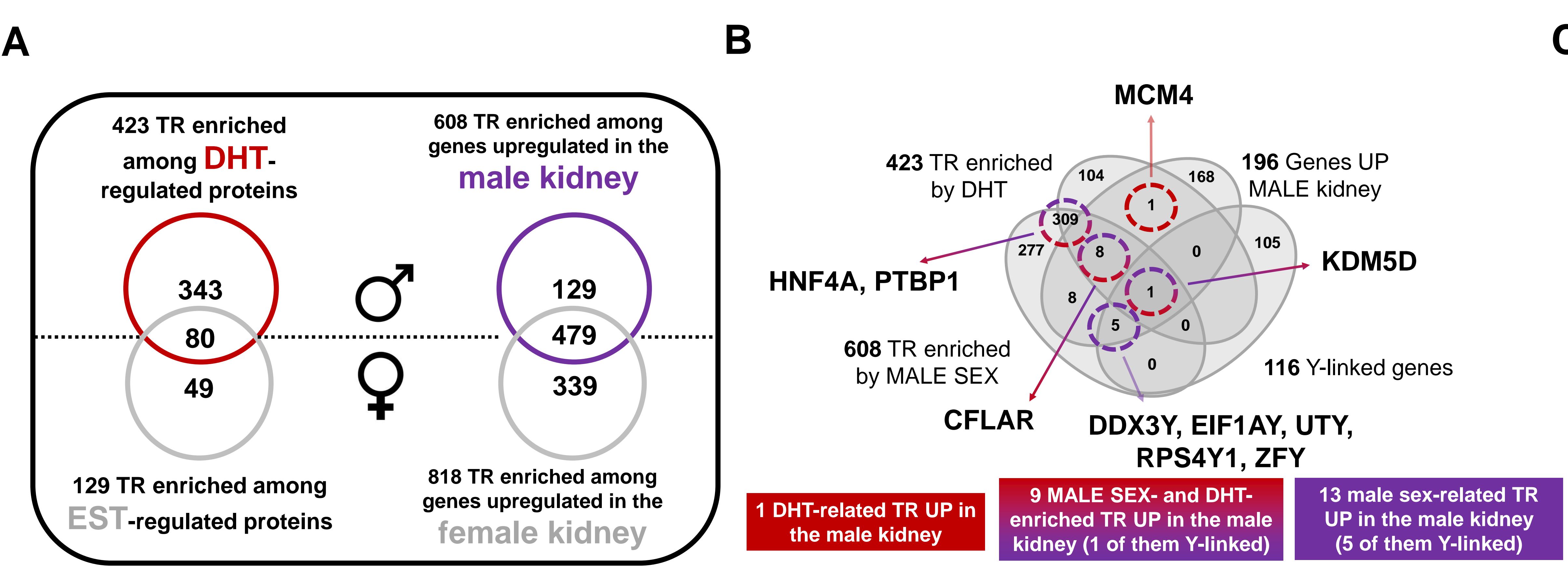

D

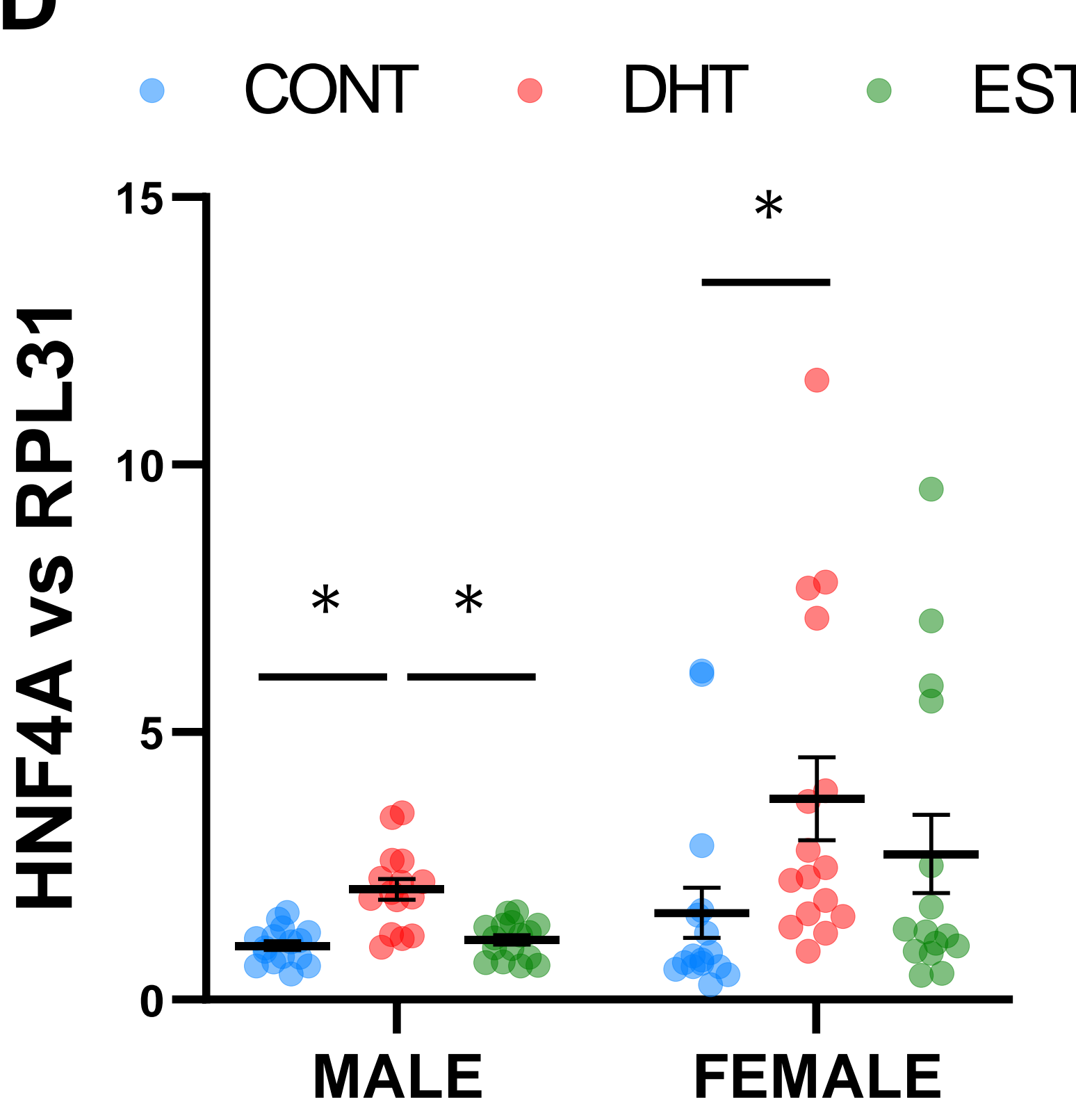

H
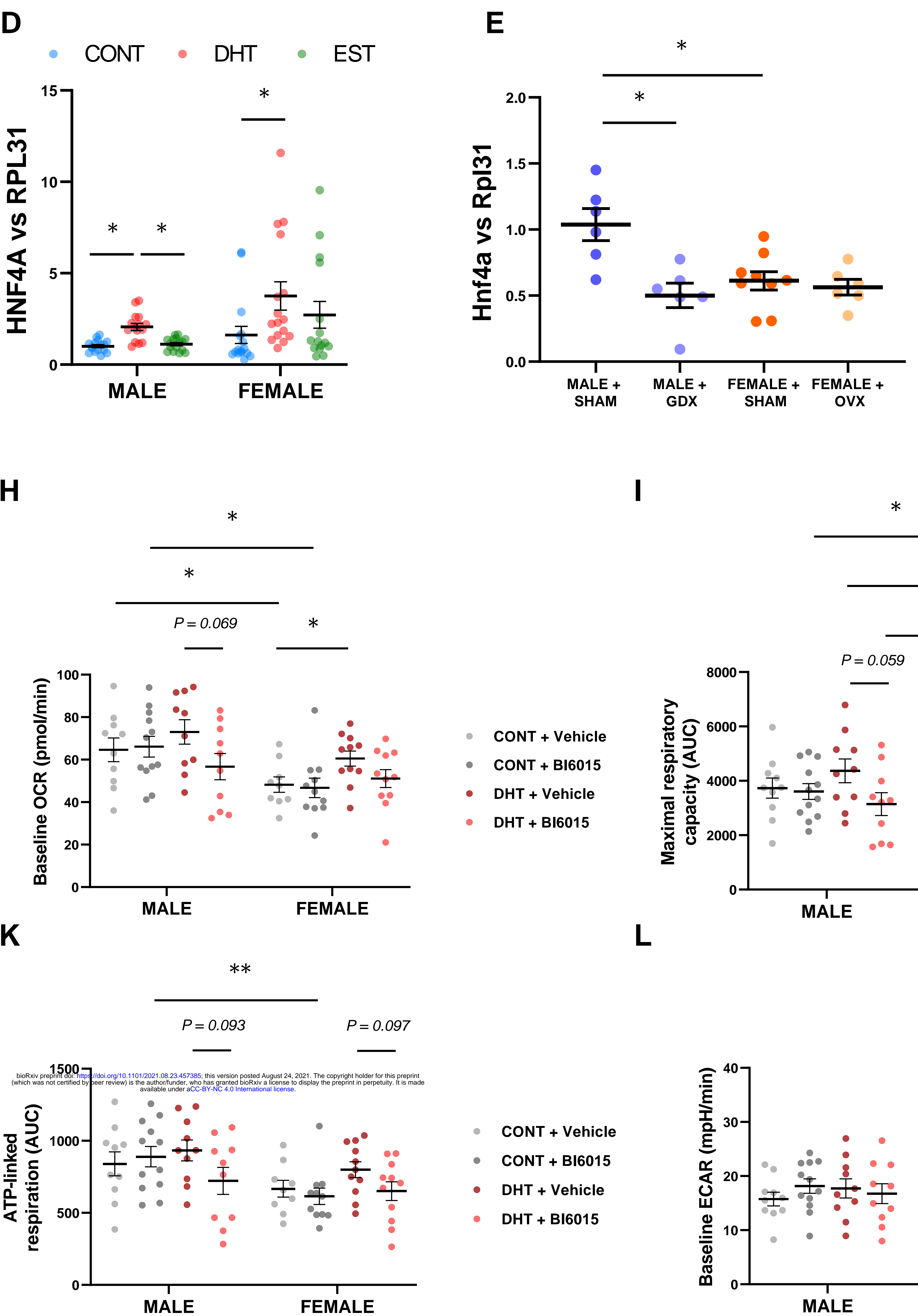

CONT + Vehicle

CONT + BI6015

- DHT + Vehicle

DHT + BI6015

$\mathbf{N}$

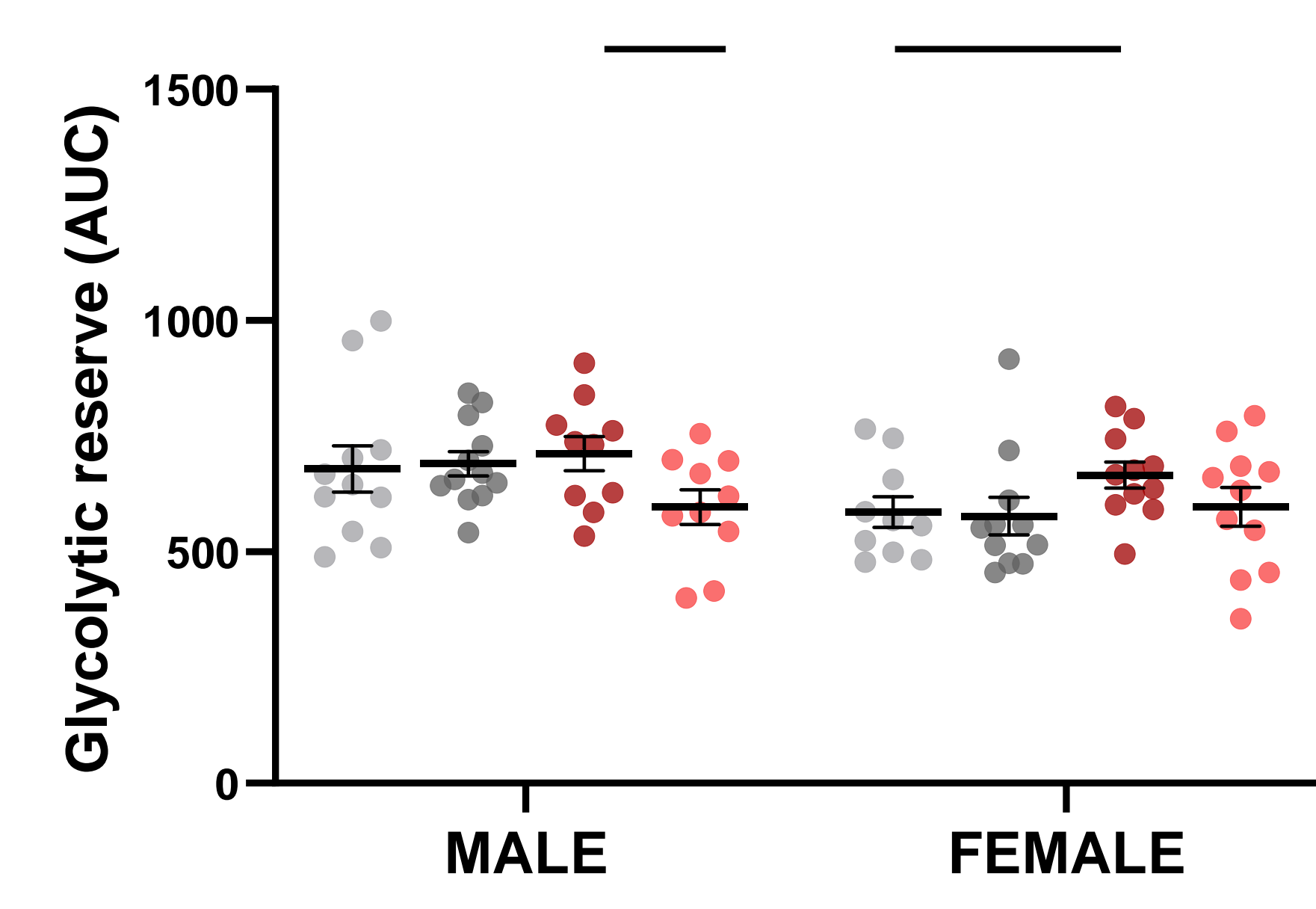

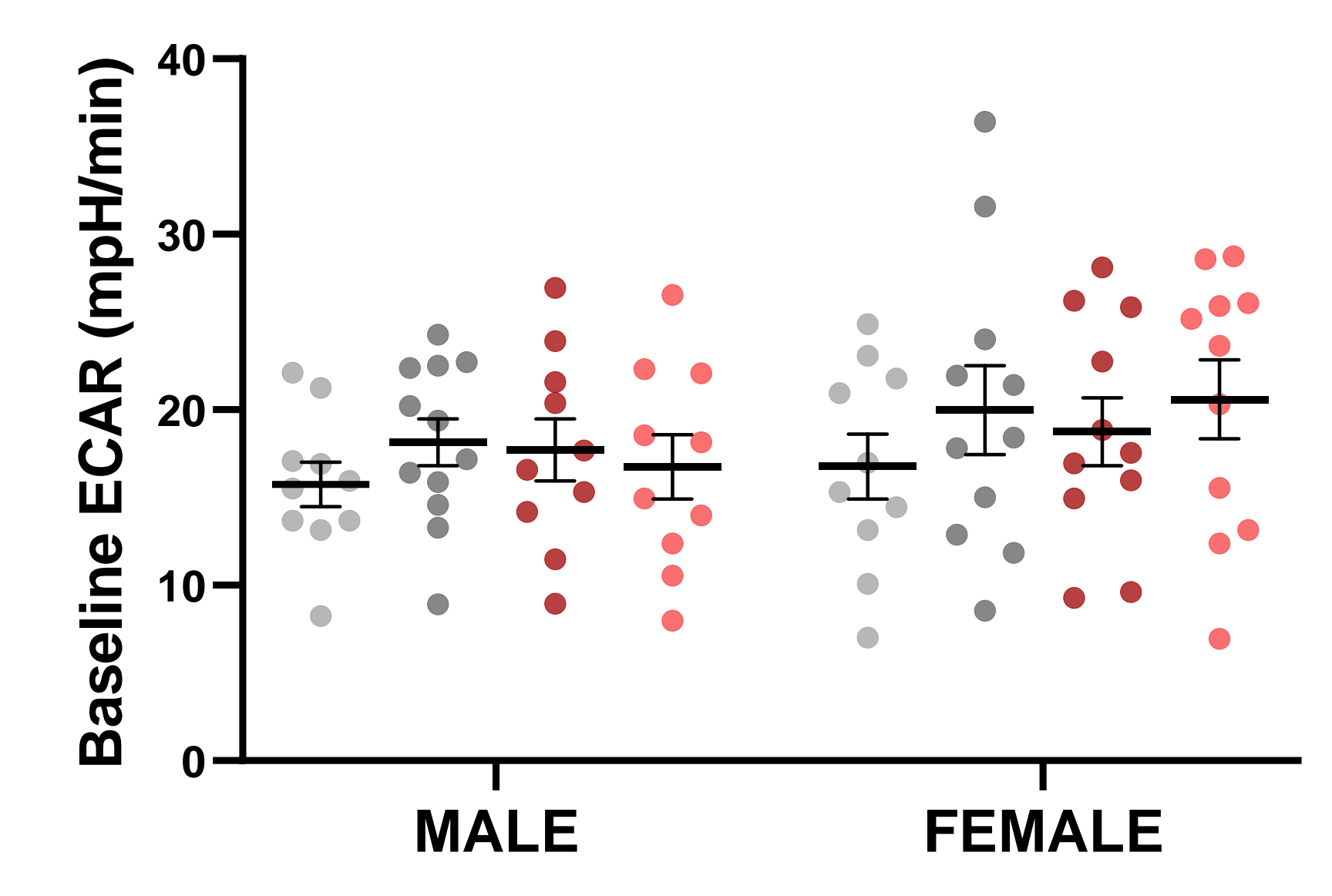

0

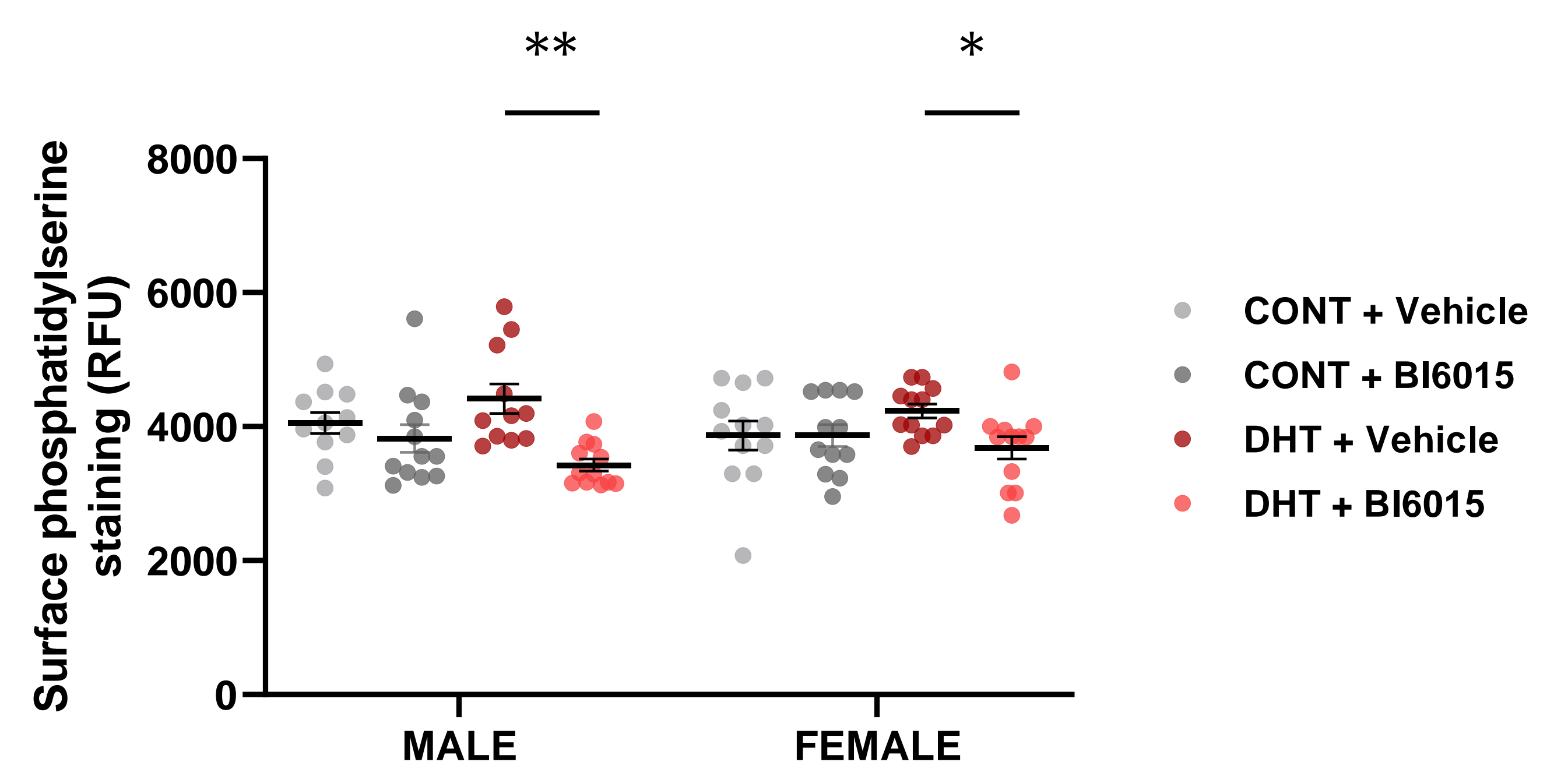

F

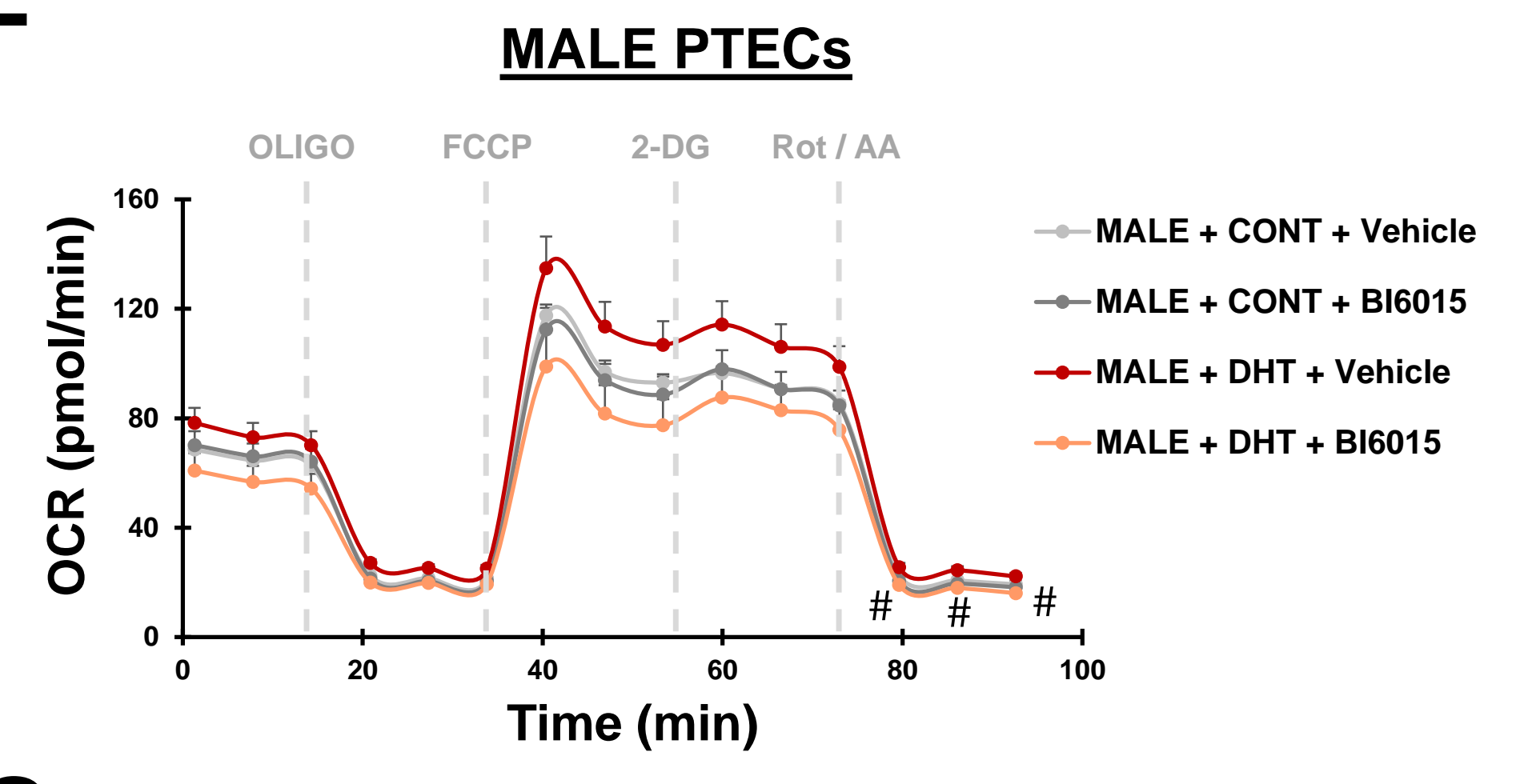

G

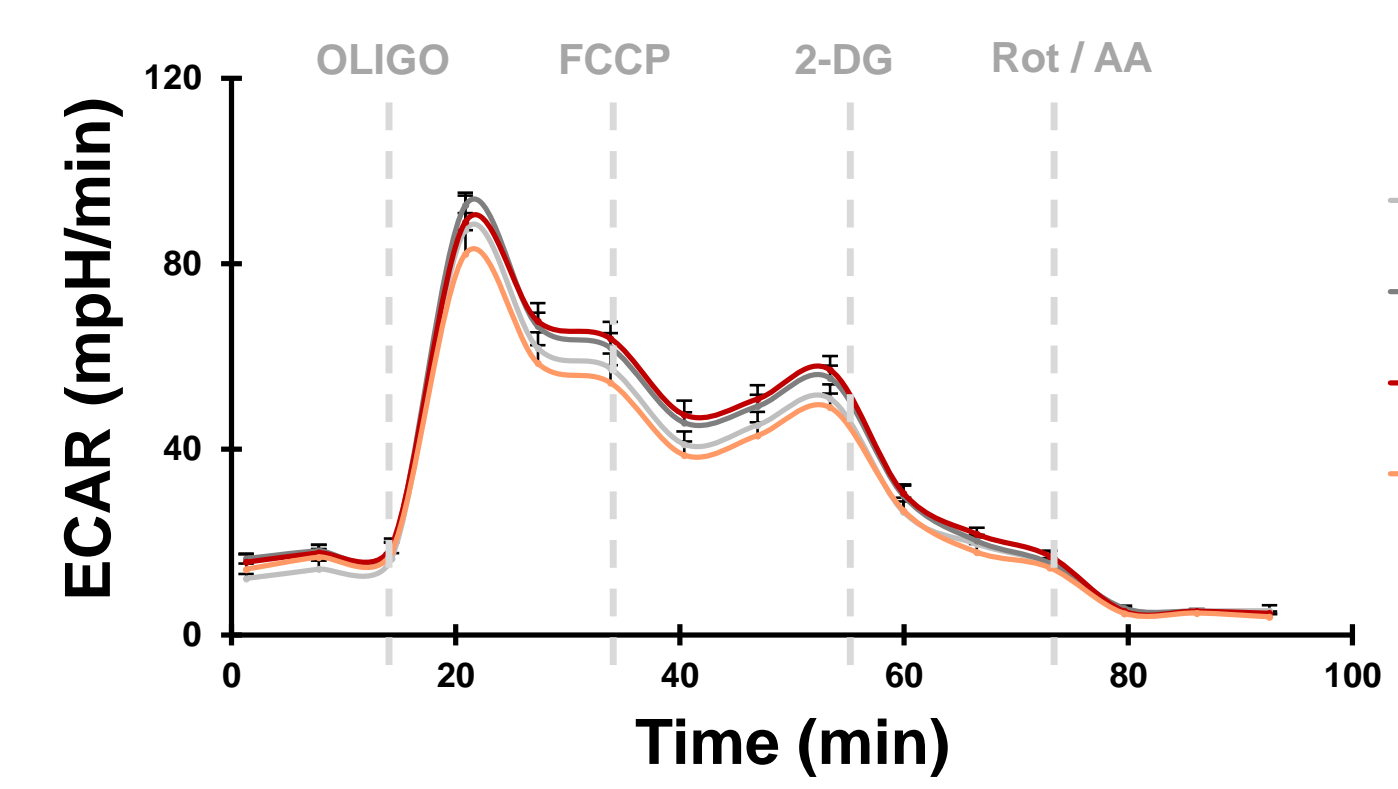

I

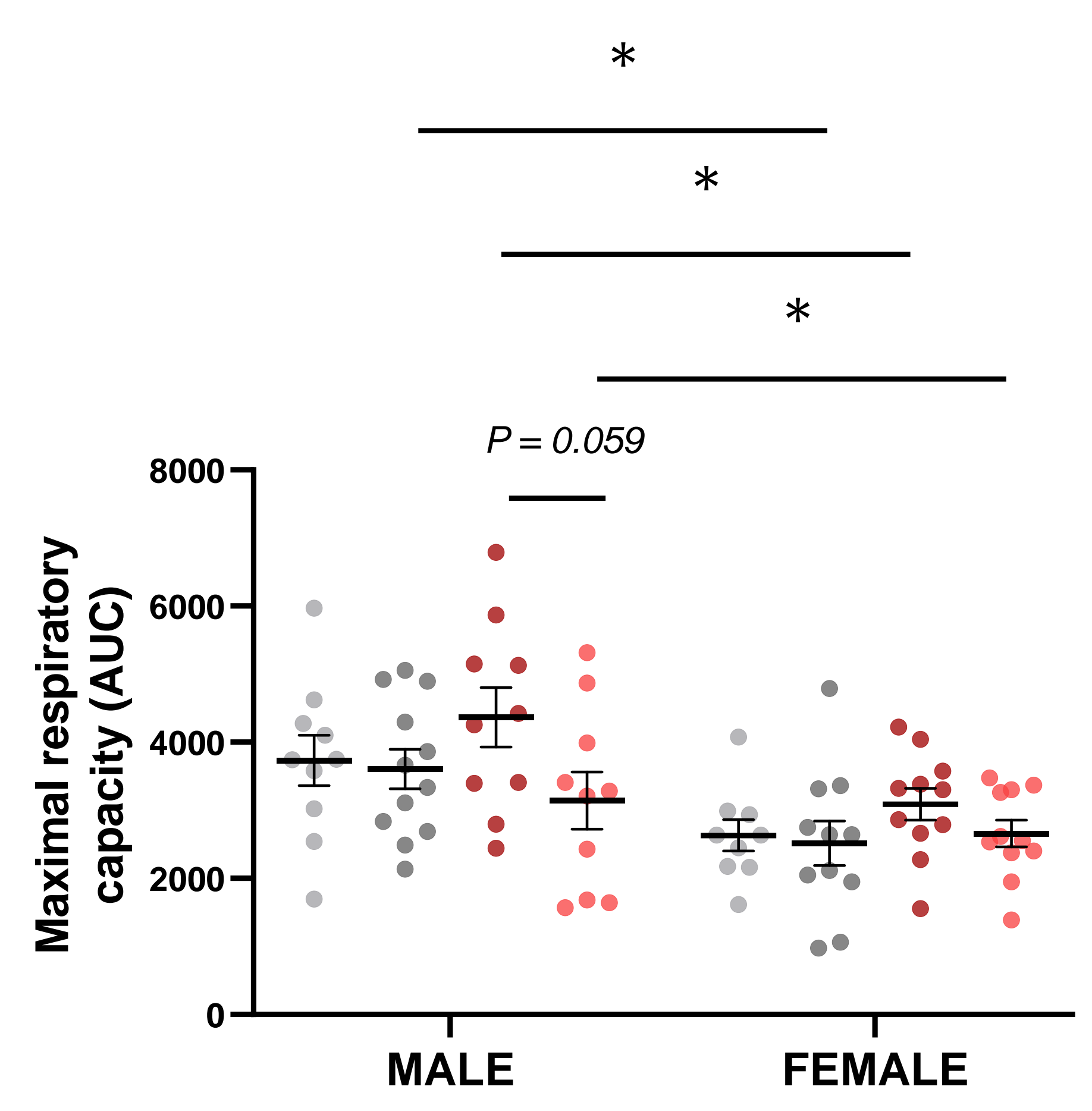

L

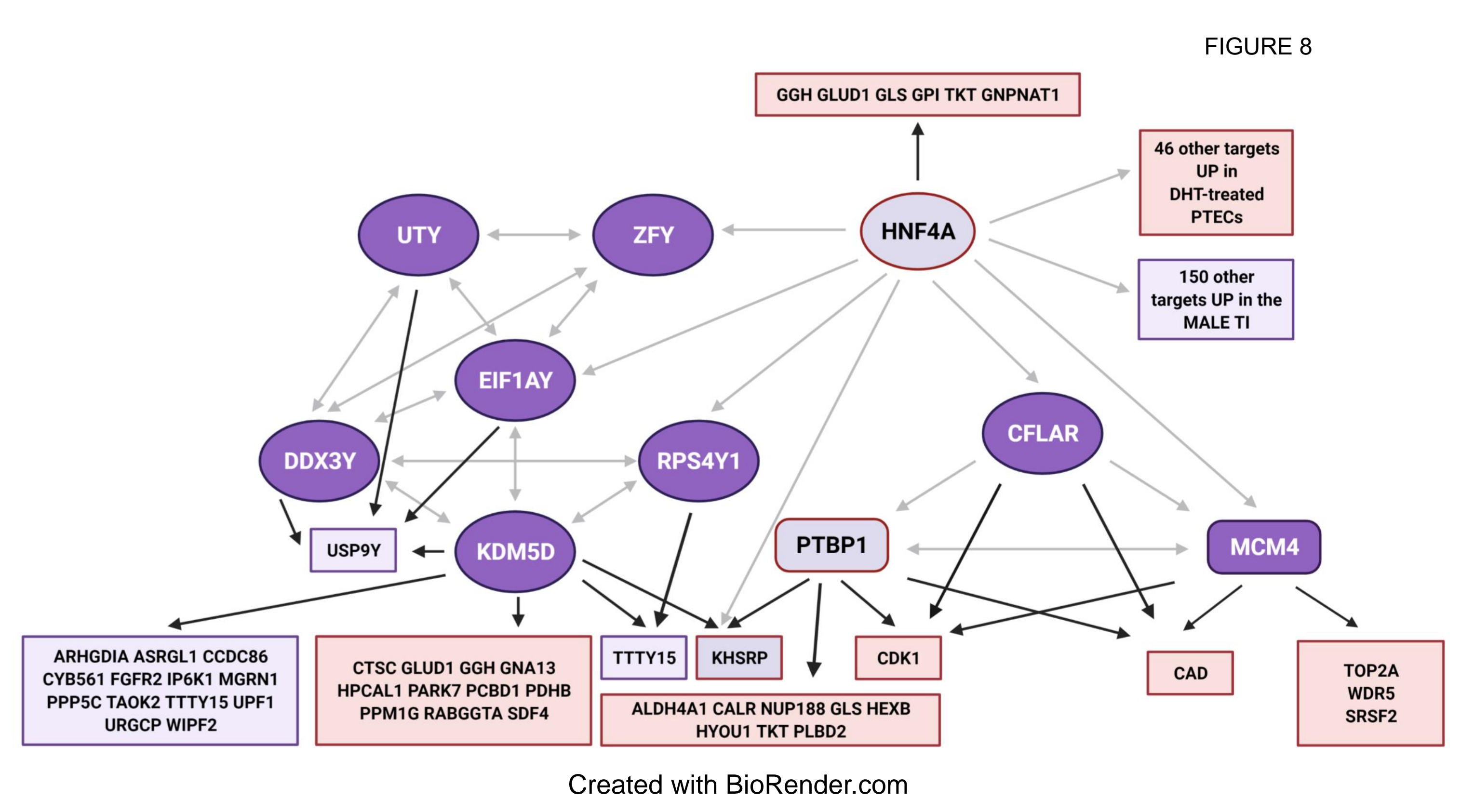

FEMALE PTECs

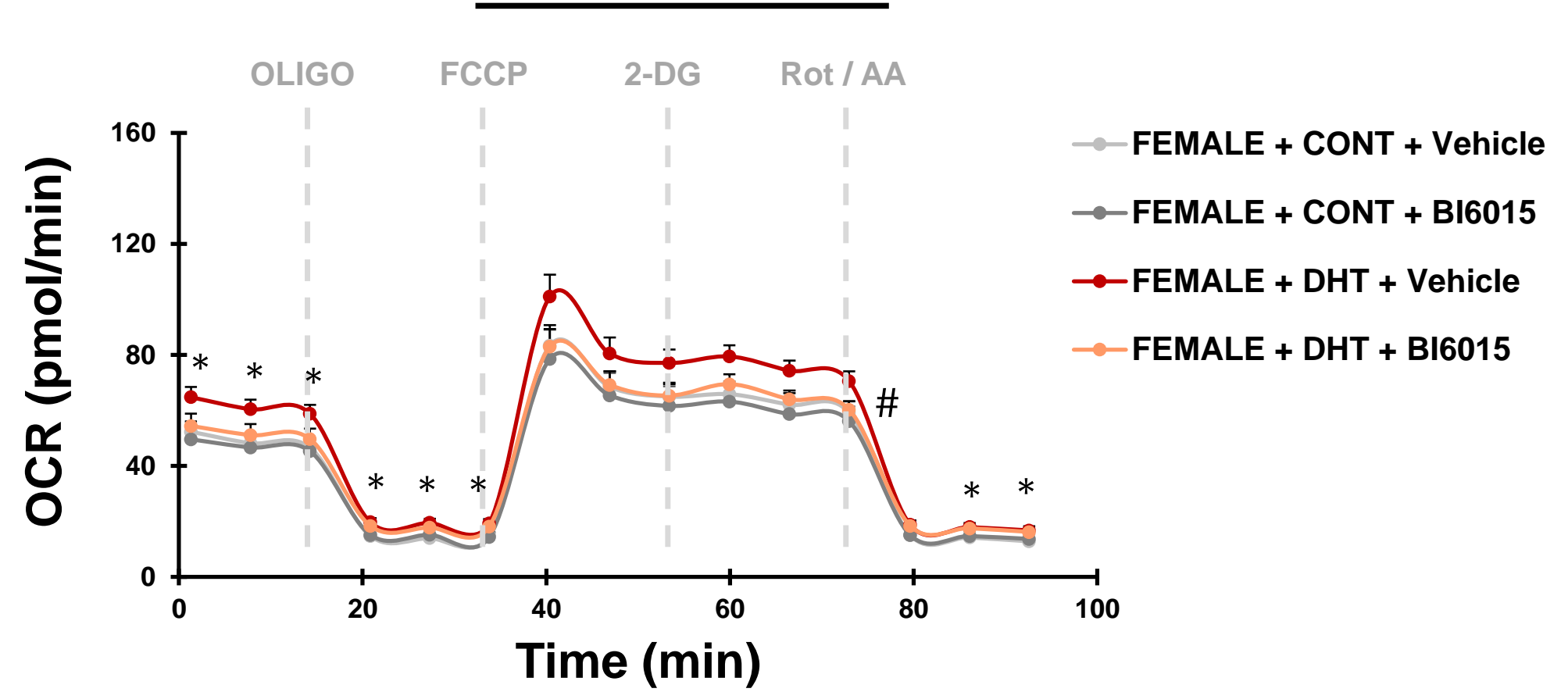

FEMALE PTECS

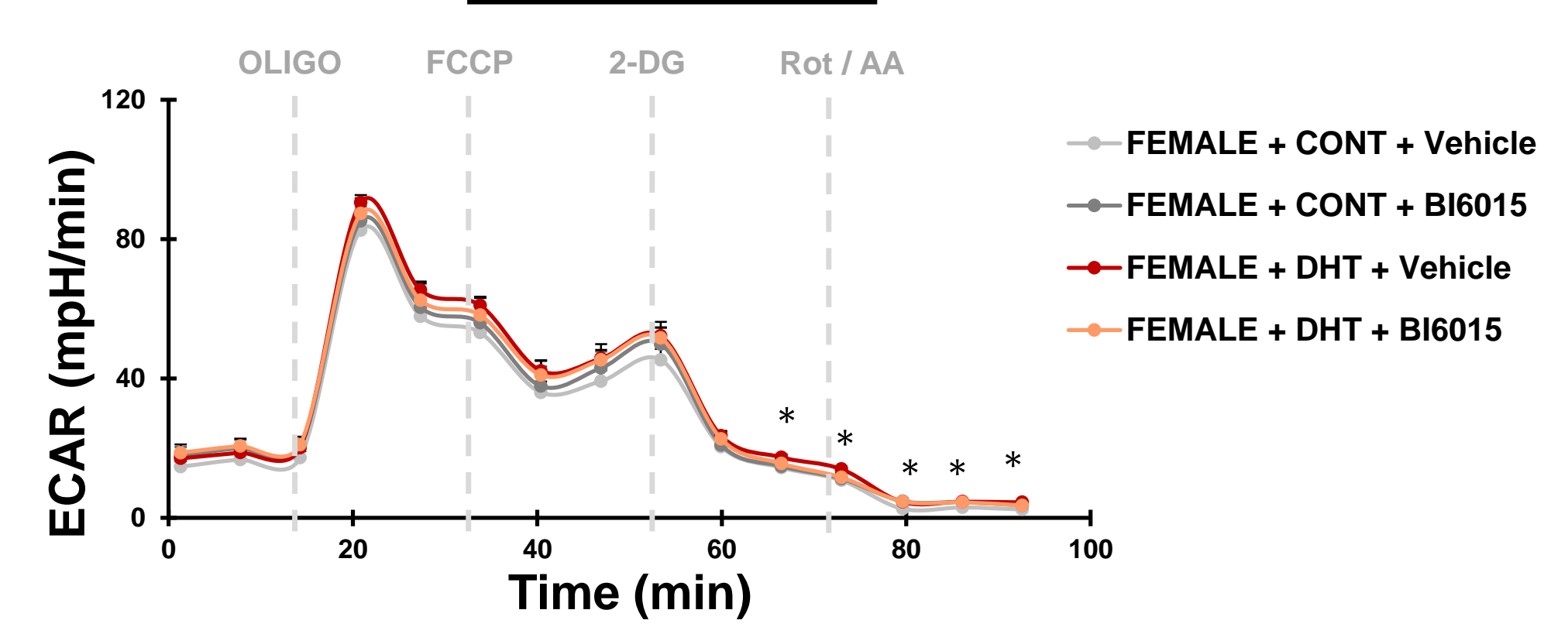

J

CONT + Vehicle

- CONT + BI6015

- DHT + Vehicle

MALE + CONT + Vehicle
MALE + CONT + B16015
MALE + DHT + Vehicle

MALE + DHT + B16015
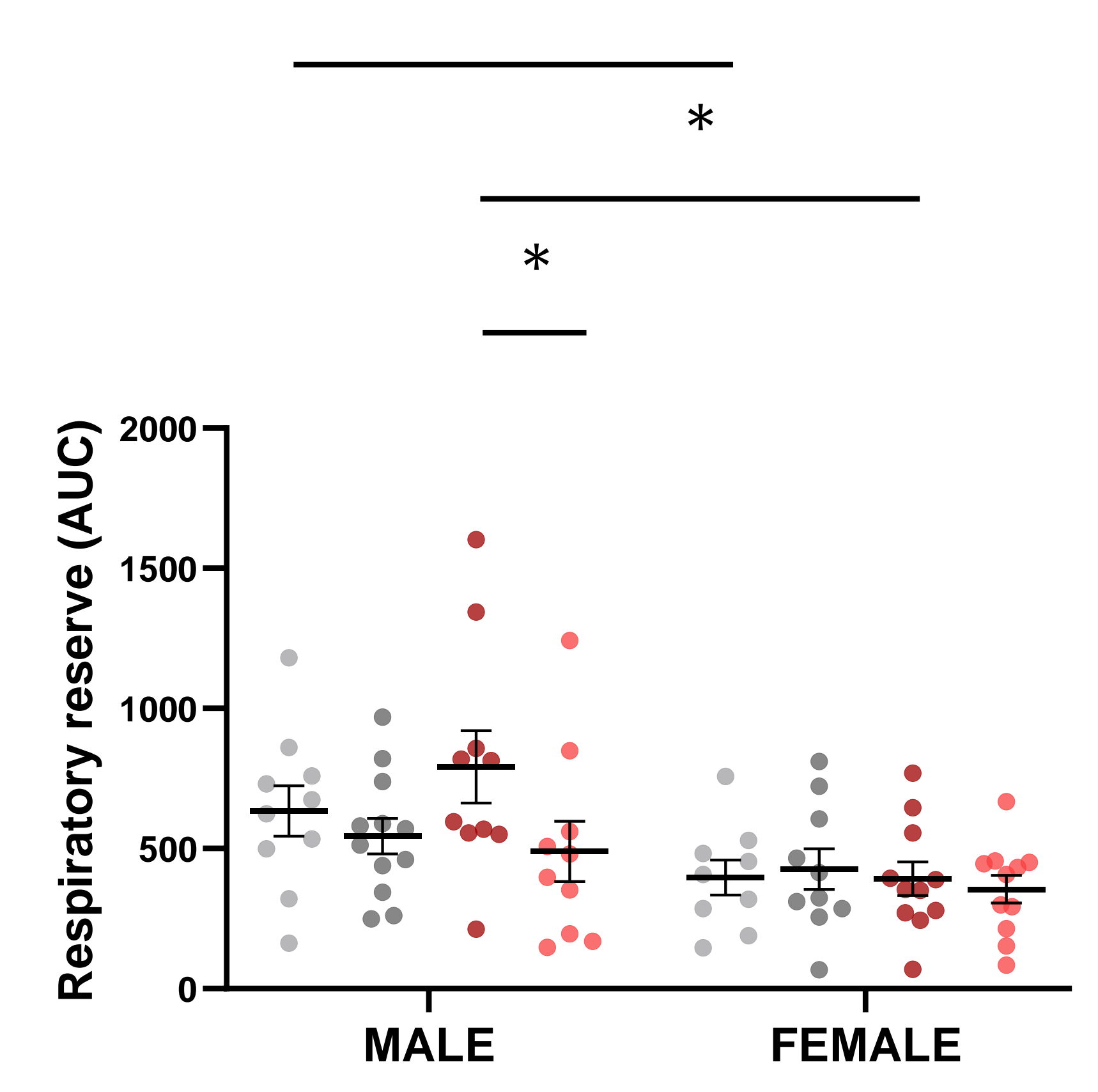

M

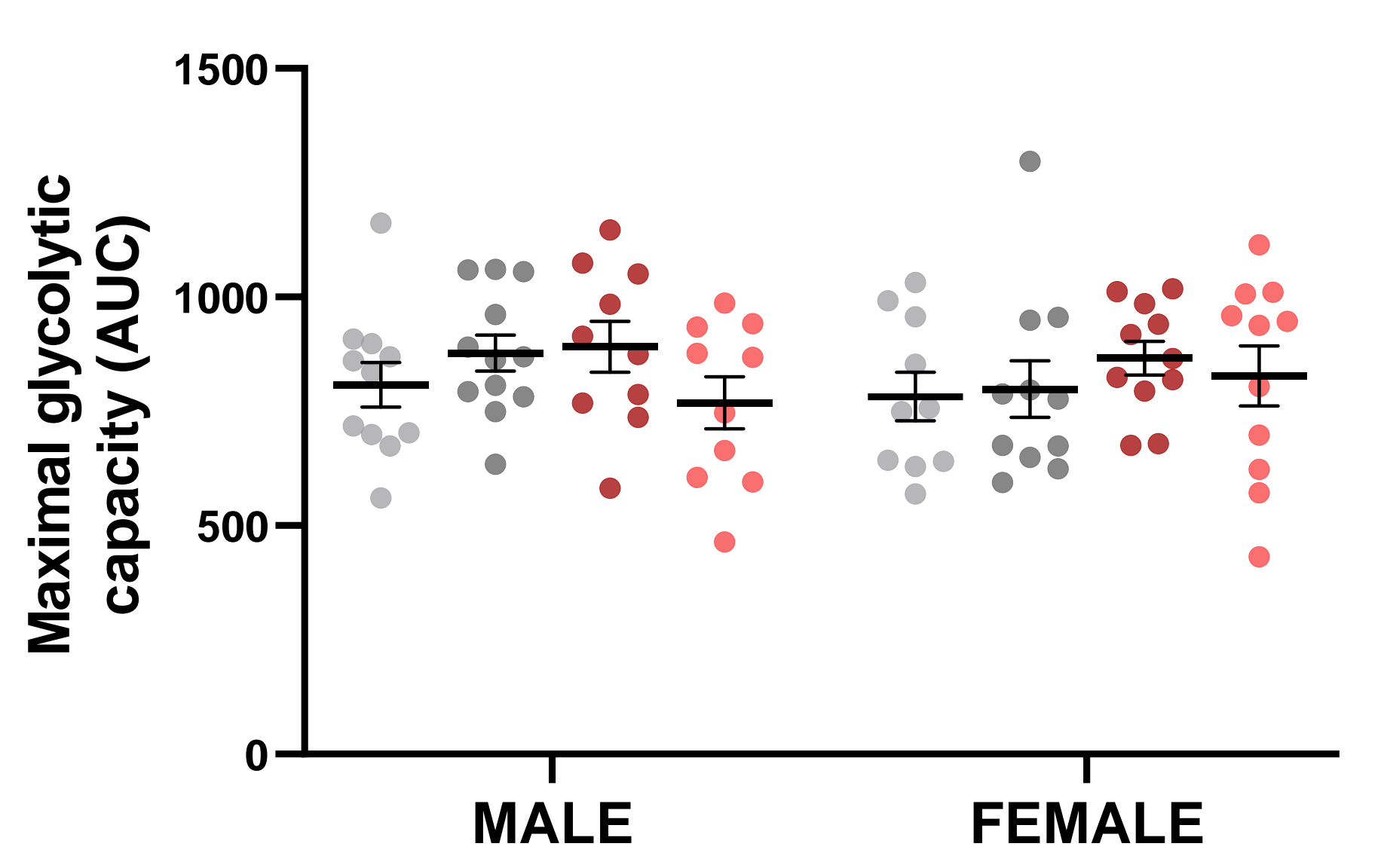

- CONT + BI6015

- DHT + Vehicle
P

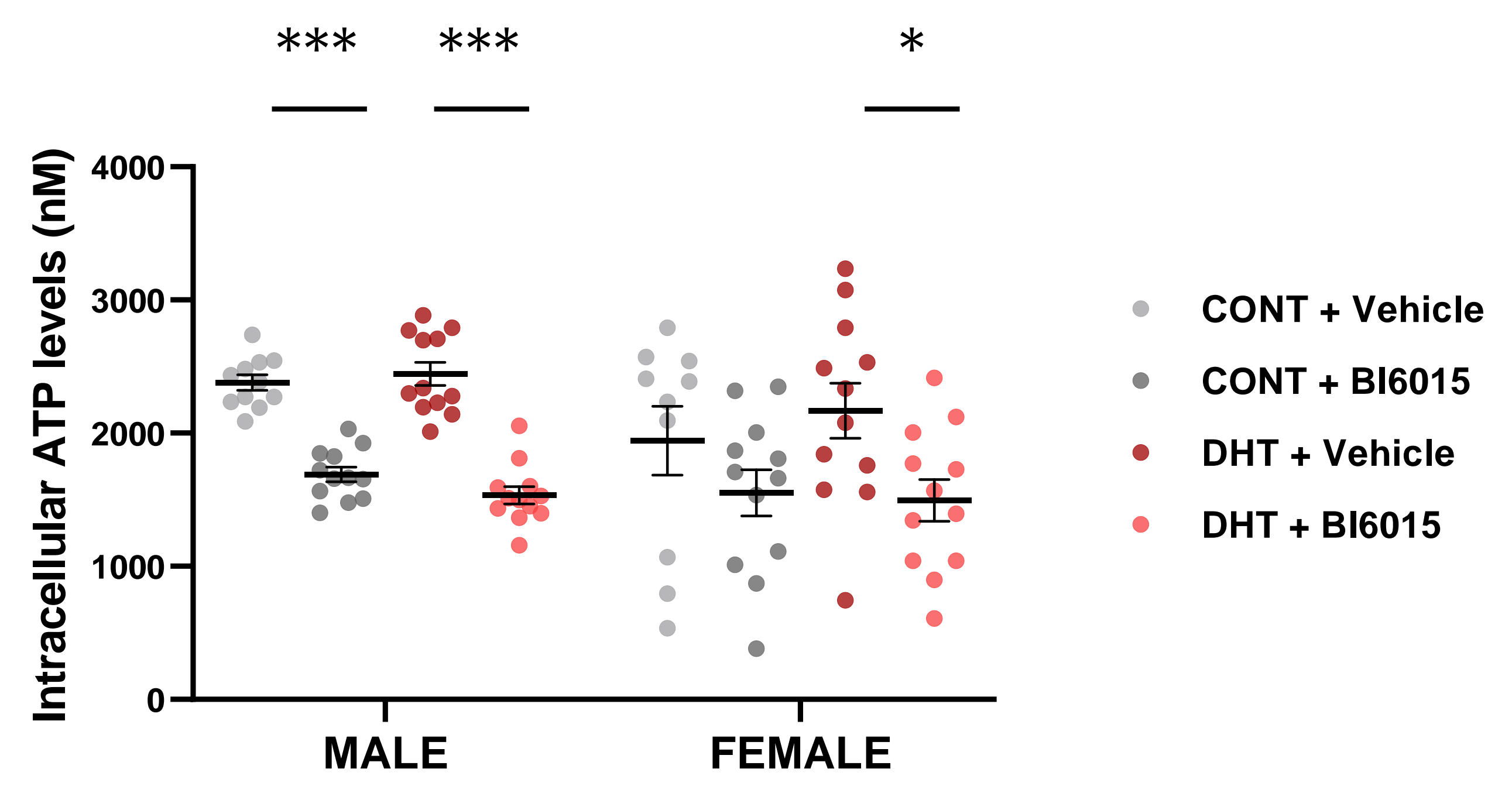


bioRxiv preprint doi: https://doi.org/10.1101/2021.08.23.457385; this version posted August 24, 2021. The copyright holder for this preprint (which was not certified by peer review) is the author/funder, who has granted bioRxiv a license to display the preprint in perpetuity. It is made

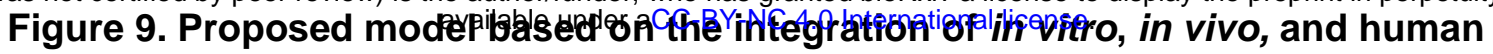

findings. Cell sex and sex hormone effects on kidney metabolism are mediated by KDM6A and HNF4A, respectively, and relate to sex differences in the levels of glucoseand glutamine-derived metabolites. In male PTECs, increased glucose consumption, mitochondrial respiration, oxidative stress, and cell death are enhanced by DHT via ARand HNF4A-dependent mechanisms. In agreement, metabolic targets of HNF4A involved in glycolysis (GPI) and glutamine anaplerosis (GLS, GLUD1) were upregulated by DHT in male PTECs ${ }^{31}$. Concordantly, increased gene levels of SLC38A3 and GLUD1 pointed towards increased glutamine anaplerosis in diabetic male kidneys. Enhanced renal utilization of glucose and glutamine in males is linked to increased levels of glutamate, malate, fumarate, succinate, GSH, GSSG, and $\gamma$-glutamyl-aminoacids, in PTECs and/or in the blood of healthy and diabetic mice and humans, suggesting a higher activation of the TCA cycle and, in consequence, a higher demand for antioxidant mechanisms. In female PTECs, metabolic homeostasis is regulated by KDM6Adependent mechanisms, and linked to increased cellular levels of pyruvate, glutamylcysteine, and cysteinylglycine, and to a higher GSH/GSSG ratio, suggestive of a favorable redox balance. Increased aminoacid excretion in non-diabetic and diabetic female individuals suggest a lower demand for aminoacid utilization in the glutathione cycle of female tissues. 


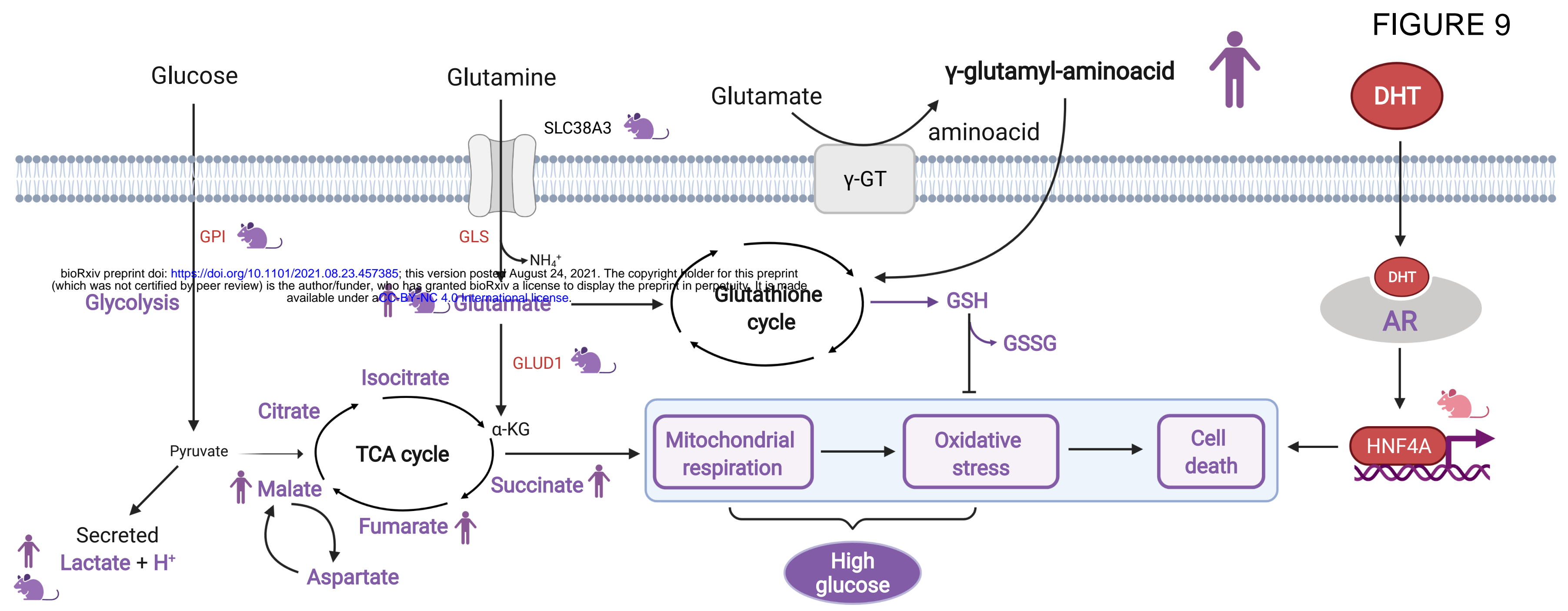

Increased in male PTECs

Increased in female PTECs
Increased by male sex
in DKD mice

Increased by female sex in DKD mice
Increased by male sex in
the human cohort

Increased by female sex in the human cohort
Increased by androgens in vivo

Targets of HNF4A increased by DHT in male PTECs (Clotet S, et al. MCP, 2017)

Regulated by KDM6A and increased in human female kidneys (Nephroseq)

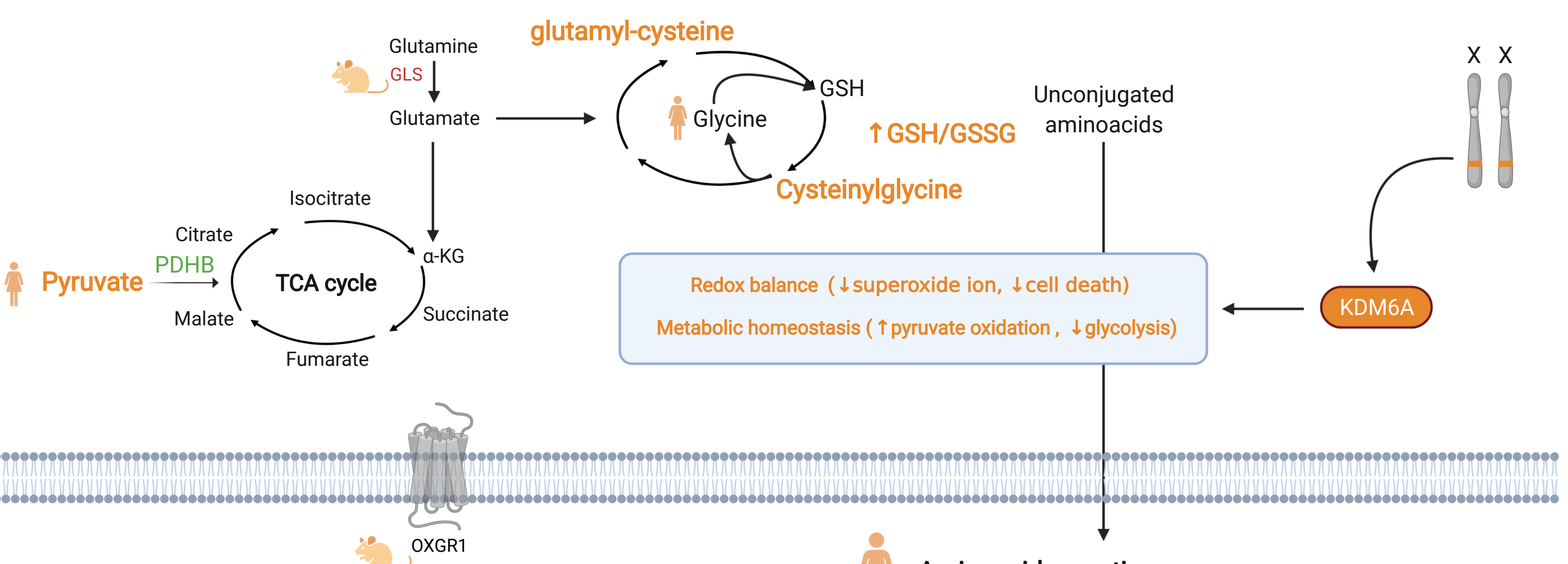


Figure S1. Sex-balanced experimental design. To study the role of cell sex and sex hormones in the setting of normal glucose, PTECs from 4 male and 5 female donors were studied in parallel. The commercial availability of male and female PTECs (Lonza) enabled the simultaneous study of cells from a maximum of 3 donors of each sex. After being serum-starved for $24 \mathrm{~h}$, PTECs were exposed to vehicle (CONT), 100nM DHT, or 100nM EST for 16-24h. A series of metabolic, kidney injury, and molecular readouts were measured after treatment $(A)$. As part of the metabolic function studies, baseline glycolysis, maximal glycolytic capacity, glycolytic reserve, and nonglycolytic acidification were assessed by calculating the estimated AUC on different sections of each ECAR curve (B). Basal respiration, ATP-linked respiration, maximal respiratory capacity, reserve capacity, proton leak, and non-mitochondrial respiration were assessed by calculating the estimated AUC on different sections of each OCR curve (C). PTECs, proximal tubular epithelial cells; AUC, area under the curve; DHT, dihydrotestosterone; EST, estradiol; ECAR, extracellular acidification rate; OCR, oxygen consumption rate; OLIGO, oligomycin; FCCP, p-trifluoromethoxy carbonyl cyanide phenyl hydrazone; 2-DG, 2-deoxyglucose; Rot, rotenone; AA: antimycin A. 


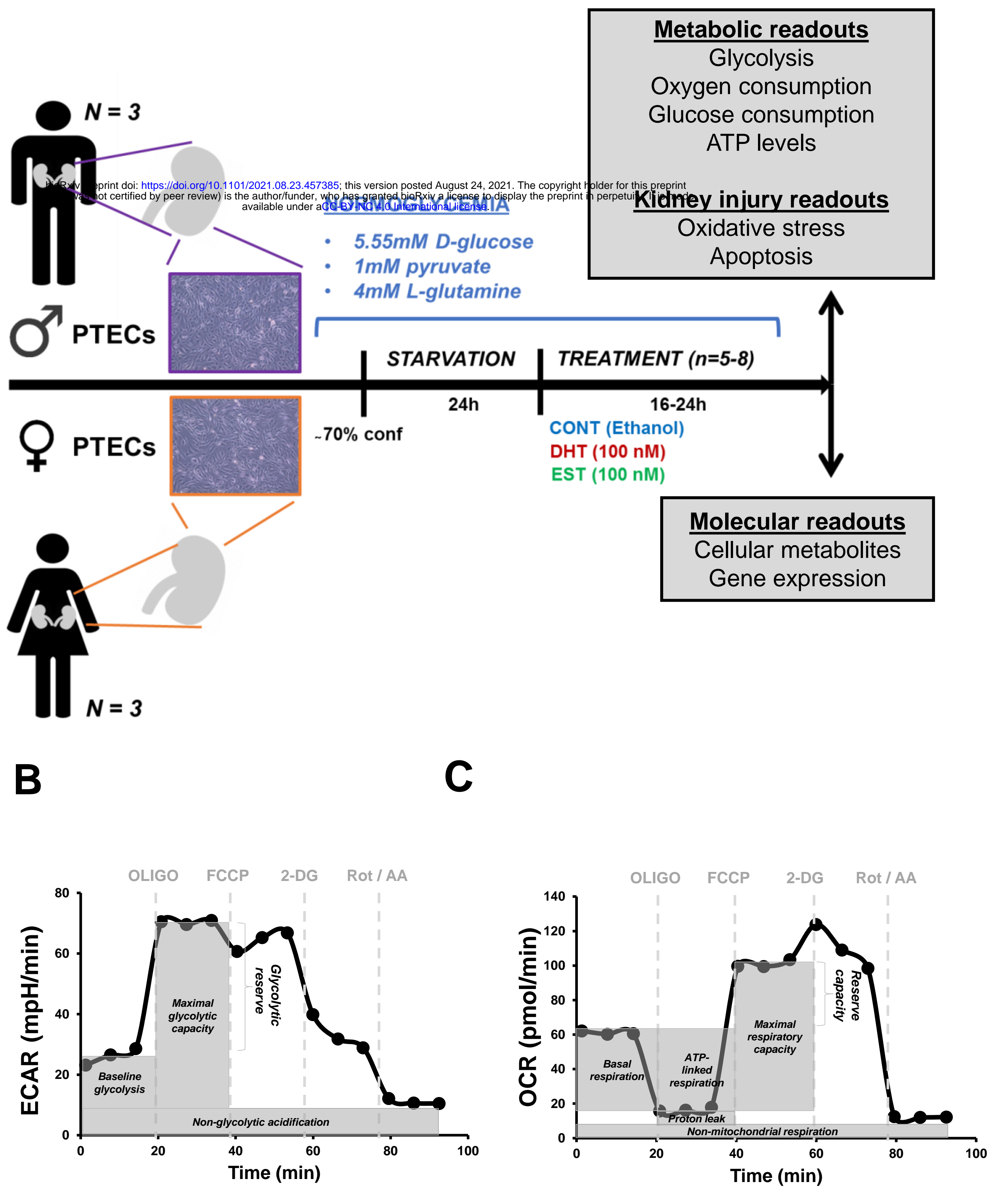


Figure S2. Effect of sex hormones on the metabolism and proliferation of male and female PTECs. Male and female PTECs were exposed to ethanol (CONT), 100nM DHT or 100nM EST for $16-24 h$ ( $n=3 /$ sex; $n=4-6 /$ treatment). Baseline $\operatorname{ECAR~}(A)$, maximal glycolytic capacity $(B)$, glycolytic reserve (C), basal respiration (D), non-mitochondrial respiration (E), and ATP-linked respiration (F) were calculated from the ECAR and OCR curves in Figure 2A. Group-to-group differences were determined using pairwise t tests for variables following a normal distribution, and Mann-Whitney tests for variables with a non-parametric distribution. ${ }^{*} p<0.05$. PTECs, proximal tubular epithelial cells; AUC, area under the curve; DHT, dihydrotestosterone; EST, estradiol; ECAR, extracellular acidification rate; OCR, oxygen consumption rate. 


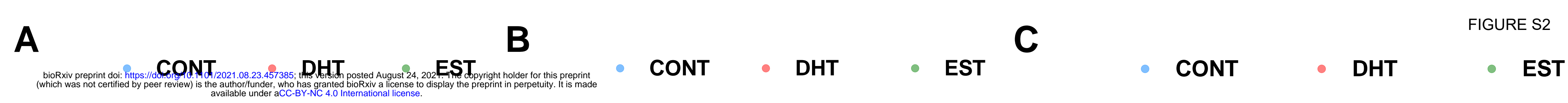
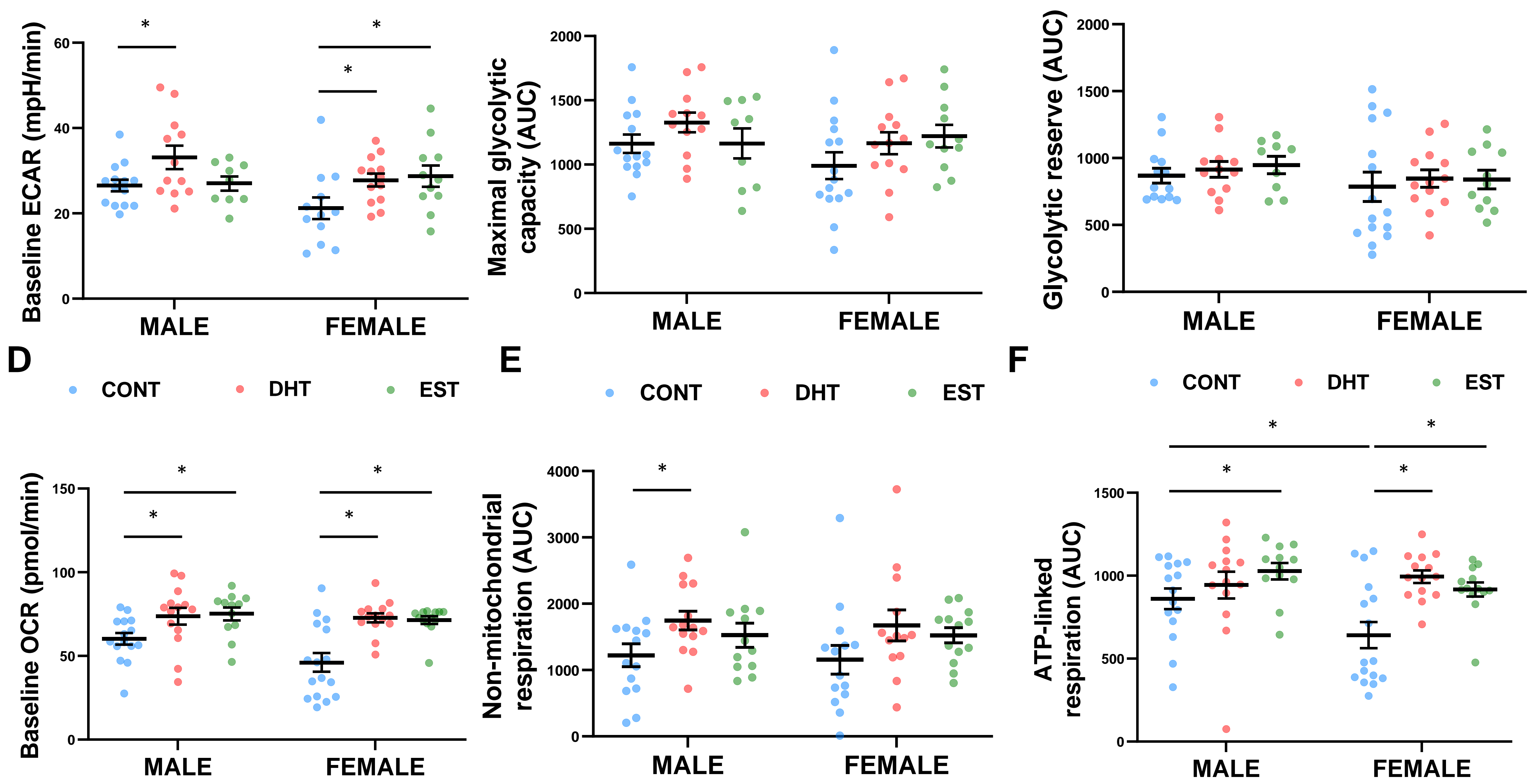
Figure S3. Effect of androgen receptor inhibition on the metabolic function of male and female PTECs. Male and female PTECs were exposed to anti-androgen treatment for $2 \mathrm{~h}$ prior to stimulation vehicle (CONT) or dihydrotestosterone (DHT) for $16 \mathrm{~h}$ ( $n=2 / \mathrm{sex} ; \mathrm{n}=4-6 /$ treatment). Two different AR inhibitors were employed: flutamide (FLUT) and enzalutamide (ENZ). FLUT is a selective antagonist of the AR, and competes with androgens like DHT for binding to AR. In turn, ENZ has a novel mode of action targeting AR signalling at three key stages: 1 ) inhibits androgen binding to AR; 2) inhibits nuclear translocation of the hormone/AR complex; and 3) inhibits binding

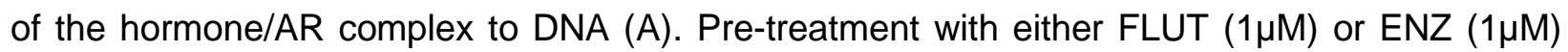
significantly prevented the DHT-induced increase in glycolysis (B) and mitochondrial respiration (C) in male PTECs and, to a lesser extent, in female PTECs. Accordingly, both FLUT and ENZ significantly reduced baseline ECAR (D), maximal glycolytic capacity (E), in male PTECs. Overall, FLUT and ENZ prevented the change towards a more energetic metabolic phenotype induced by DHT in male PTECs. Although to a lesser extent, this effect was also observed in female PTECs (I). In addition, pre-treatment with FLUT and ENZ reduced the intracellular levels of superoxide ion in DHT-treated male and female PTECs $(J)$. In line with their increased susceptibility to DHT treatment and AR inhibition, male PTECs displayed a significant increase in the gene expression of $\mathrm{AR}$, compared to female PTECs (K). Group-to-group differences were determined using pairwise t tests for variables following a normal distribution, and Mann-Whitney tests for variables with a non-parametric distribution. ${ }^{*} \mathrm{p}<0.05 ;{ }^{* *} \mathrm{p}<0.01 ;{ }^{* *} \mathrm{p}<0.001$. PTECs, proximal tubular epithelial cells; AUC, area under the curve; DHT, dihydrotestosterone; EST, estradiol; ECAR, extracellular acidification rate; OCR, oxygen consumption rate; AR, androgen receptor; HPRT1, hypoxanthine-guanine phosphoribosyltransferase; RFU, relative fluorescence units. 
Figure S4. Effects of high glucose on the metabolism of male and female PTECs exposed to sex hormones.

Male and female PTECs were serum starved and treated with vehicle (CONT), 100nM DHT or 100nM EST for 16h, prior to the exposure to high glucose. ECAR and OCR were monitored to assess glycolysis and mitochondrial function, respectively, at baseline and after $2 \mathrm{~h}, 48 \mathrm{~h}$, and $96 \mathrm{~h}$ of exposure to high glucose (25mM glucose; $(n=2 /$ sex; $n=4-6 /$ time point). DHT and EST concentrations were adjusted to $1 \mathrm{nM}$ during the high glucose treatment. The figure shows the evolution over time of key metabolic parameters: baseline glycolysis (A), maximal glycolytic capacity (B), basal respiration (C), ATP-linked respiration (D), maximal respiratory capacity (E), reserve respiratory capacity $(F)$, and non-mitochondrial respiration $(G)$. The evolution of the metabolic phenotype of male $(\mathrm{H})$ and female PTECs (I) exposed to high glucose and sex hormones was visualized by plotting ECAR on the $\mathrm{X}$ axis and OCR on the $\mathrm{Y}$ axis. Intracellular levels of ATP $(\mathrm{J})$ and superoxide ion $(\mathrm{K})$ were also measured after $96 \mathrm{~h}$ of exposure to high glucose. In panels A-G, a t test was used at each time point to assess statistical significance between the two groups. In panels J-K, group-to-group differences were determined using pairwise t tests for variables following a normal distribution, and Mann-Whitney tests for variables with a non-parametric distribution. ${ }^{*} p<0.05$ vs female PTECs; $\$ p<0.05$ vs CONT; $\# p<0.05$ vs DHT. PTECs, proximal tubular epithelial cells; AUC, area under the curve; DHT, dihydrotestosterone; EST, estradiol; HG, high glucose; ECAR, extracellular acidification rate; OCR, oxygen consumption rate. 
-Female + CONT A

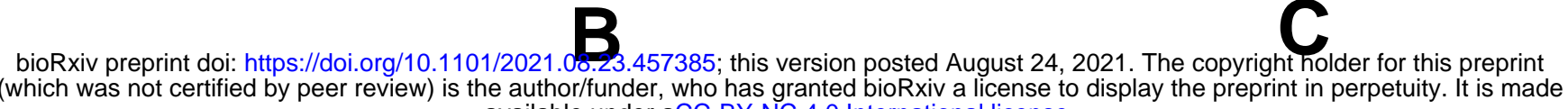

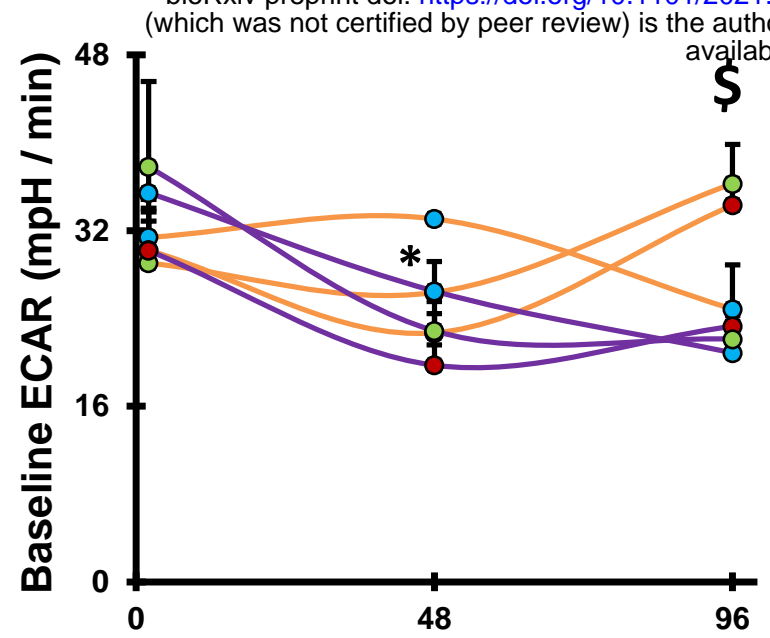

Time of Hyperglycemia (h)

$\mathbf{F}$
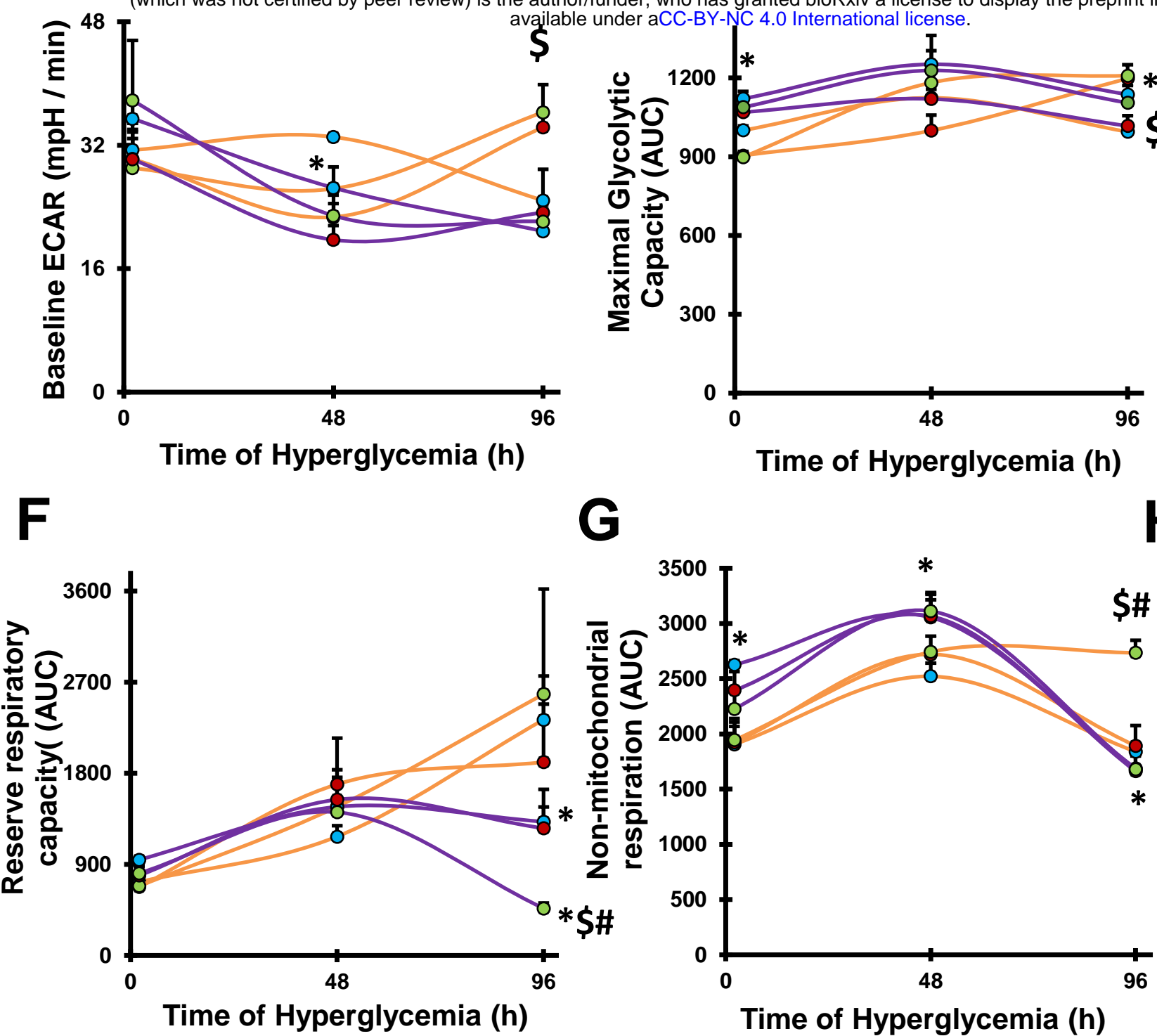

G
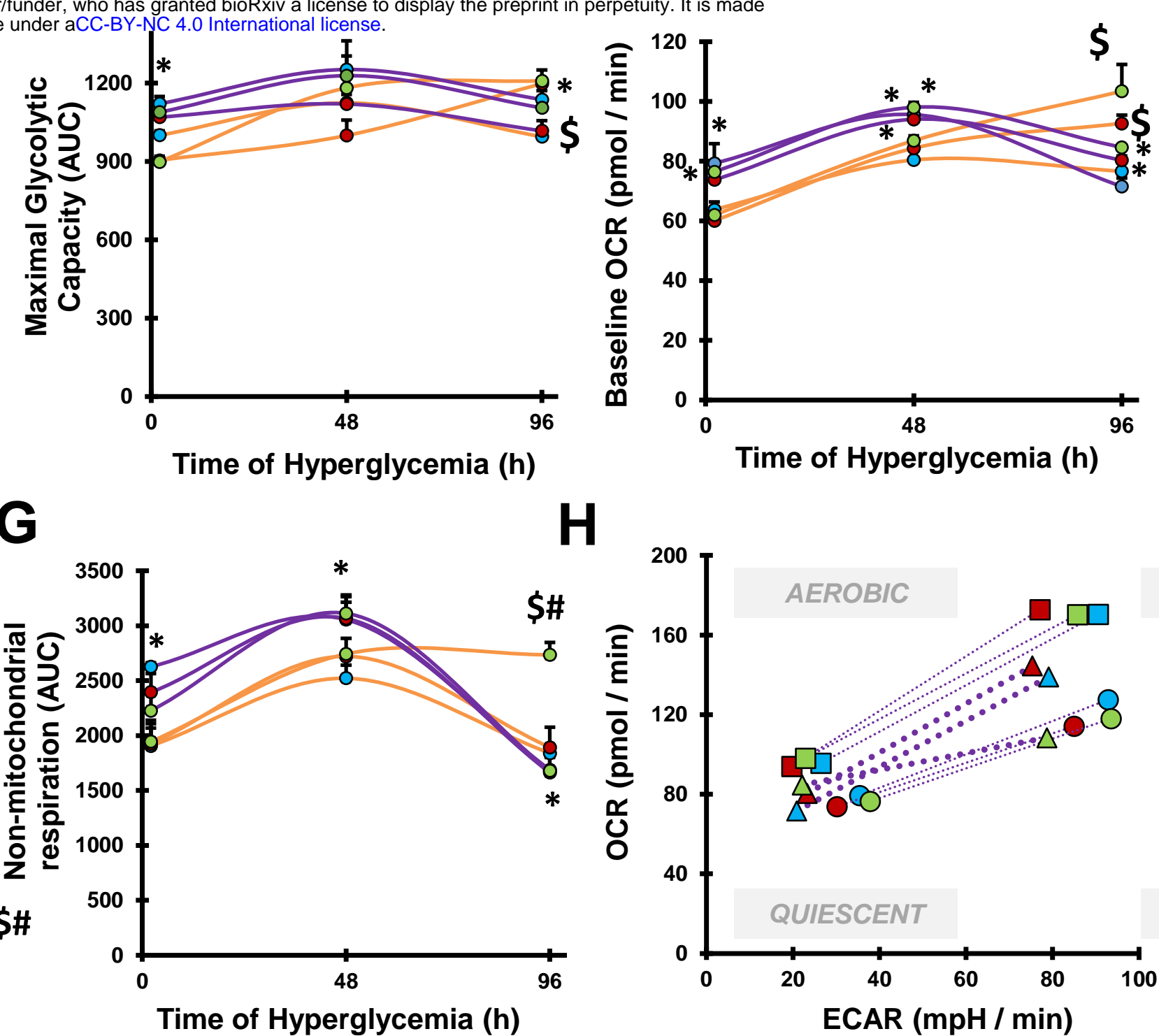

H

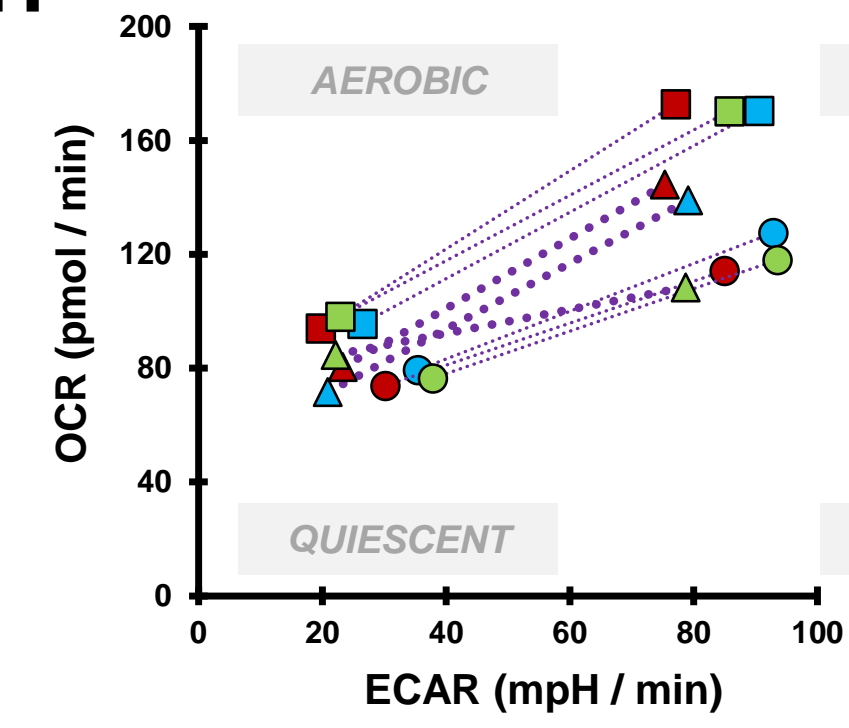

D $\rightarrow$ Male + DHT

E

FlGURE S4
-o-Male + EST
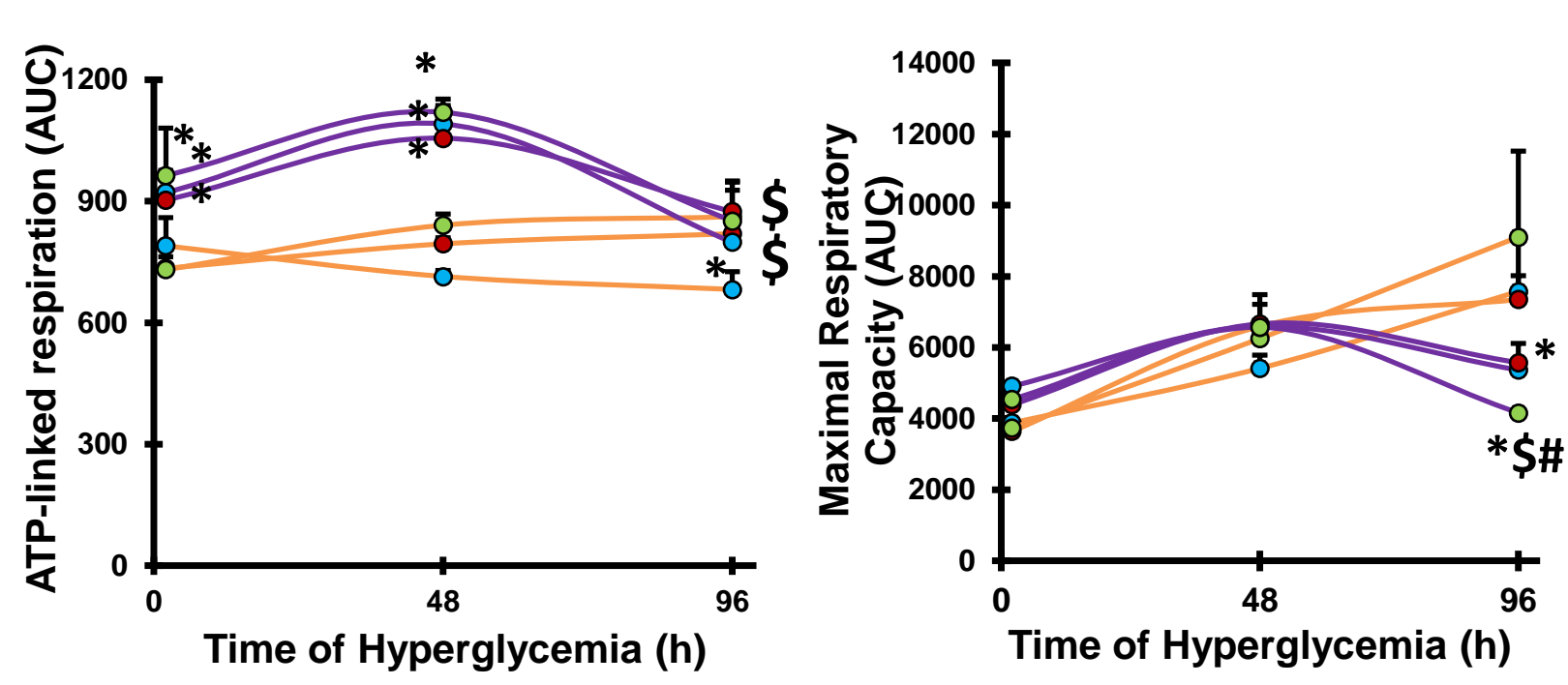

I

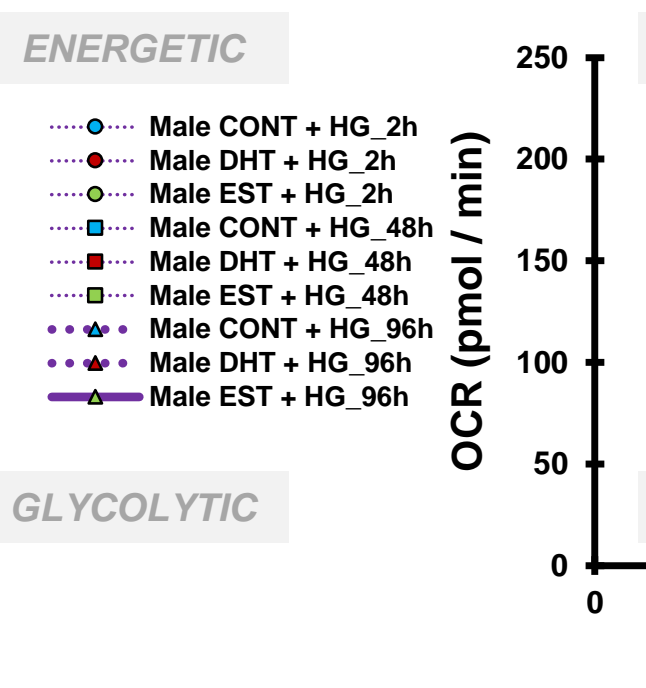

AEROBIC

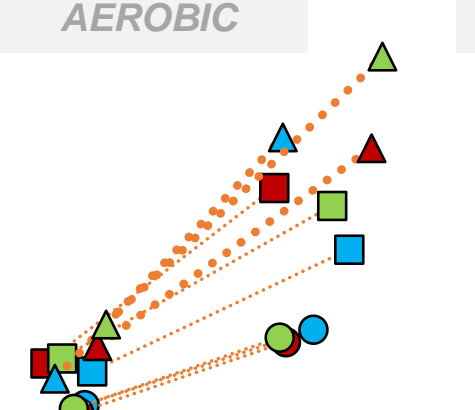

ENERGETIC

0.... Female CONT + HG_2h .... Female DHT + HG_2h

O.... Female EST + HG_2h

.... Female DHT + HG 48 h

.... Female EST + HG_48h

D.. Female CONT + HG_96h 2
0. Female DHT + HG_96h

QUIESCENT GLYCOLYTIC

ECAR $(\mathrm{mpH} / \mathrm{min})$

K

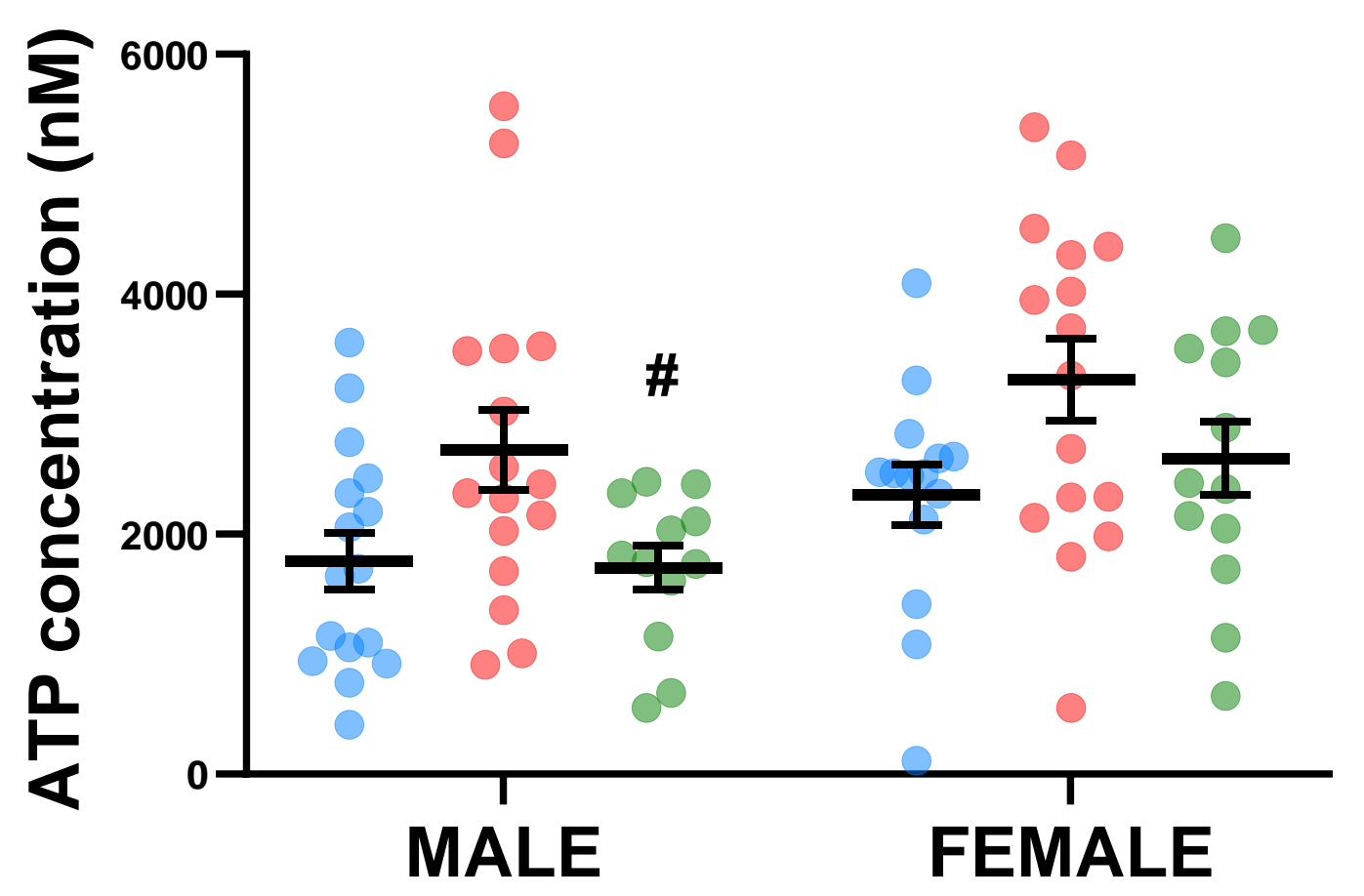

- CONT

- DHT

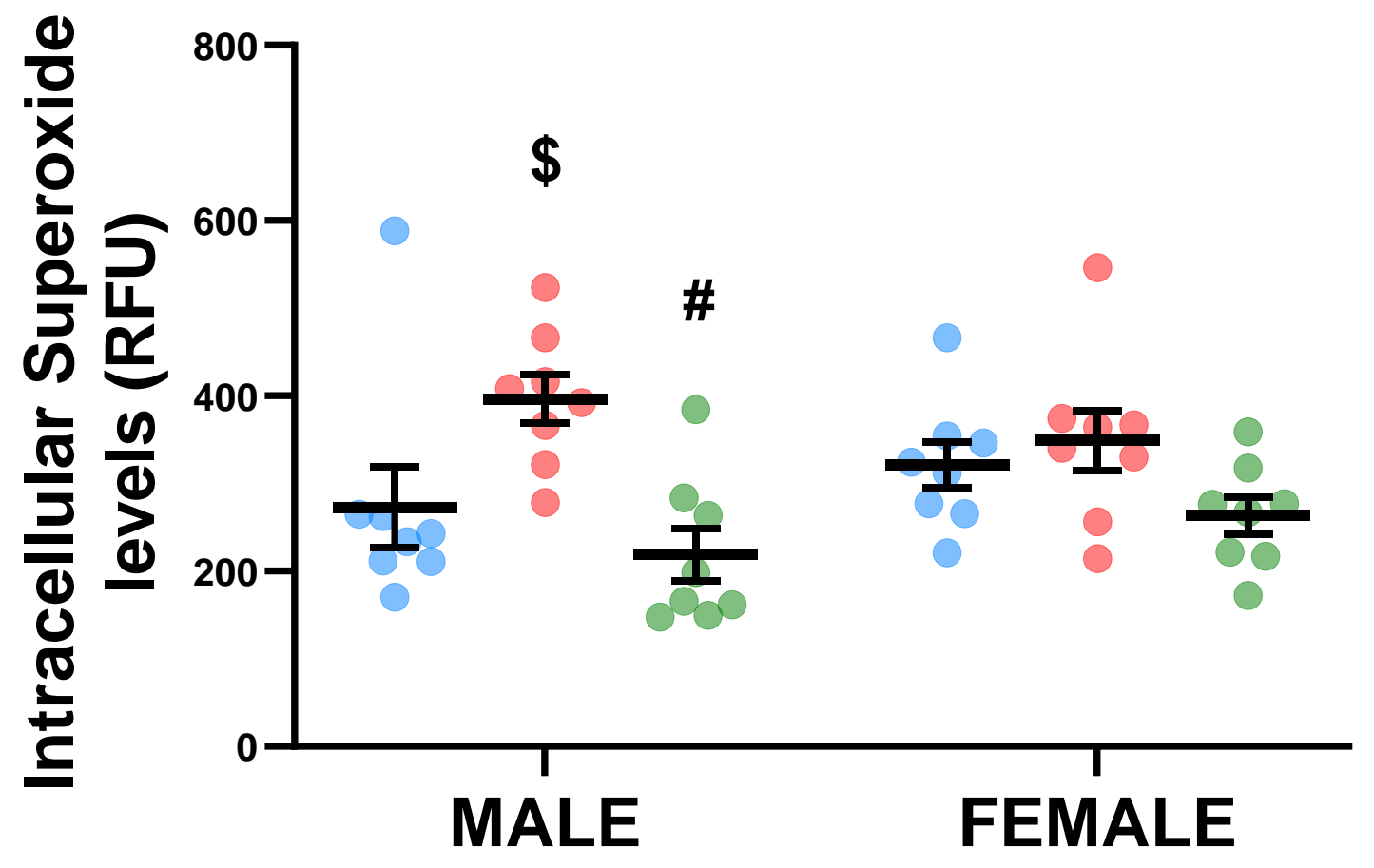

- CONT

- EST 
Figure S5. Sex-based differences on urine metabolites and kidney expression of genes related to glucose and glutamine metabolism in vivo.

The urinary excretion of glucose, lactate, glutamine, and glutamate was measured in 30-weekold male mice previously subjected to gonadectomy (GDX). Metabolite levels in the urine of GDX male mice were compared to those in the urine of sham-operated males and intact females (A). Male mice displayed a reduction in the urinary levels of all four metabolites, in comparison to females, and this reduction was prevented by GDX (B) ( $n=5-8$ animals/group). The kidney gene expression of Slc38a3, Gls, Glud1, and Oxgr1 was measured in 16-week-old male and female, healthy and type 1 diabetic (Akita) mice, and normalized to Hprt1 (C,D) (n=5-6 animals/group). Group-to-group differences were determined using pairwise t tests for variables following a normal distribution, and Mann-Whitney tests for variables with a non-parametric distribution. * $\mathrm{p}<0.05$; ${ }^{* *} p<0.01 ;{ }^{* *} p<0.001$. TCA, tricarboxylic acid; $\alpha-K G$, alpha-ketoglutarate; SLC38A3, sodiumcoupled neutral amino acid transporter 3; GLS, glutaminase; GLUD1, glutamate dehydrogenase; OXGR1, alpha-ketoglutarate receptor 1; HPRT1, hypoxanthine-guanine phosphoribosyltransferase. The illustration in panel C was created with BioRender.com. 


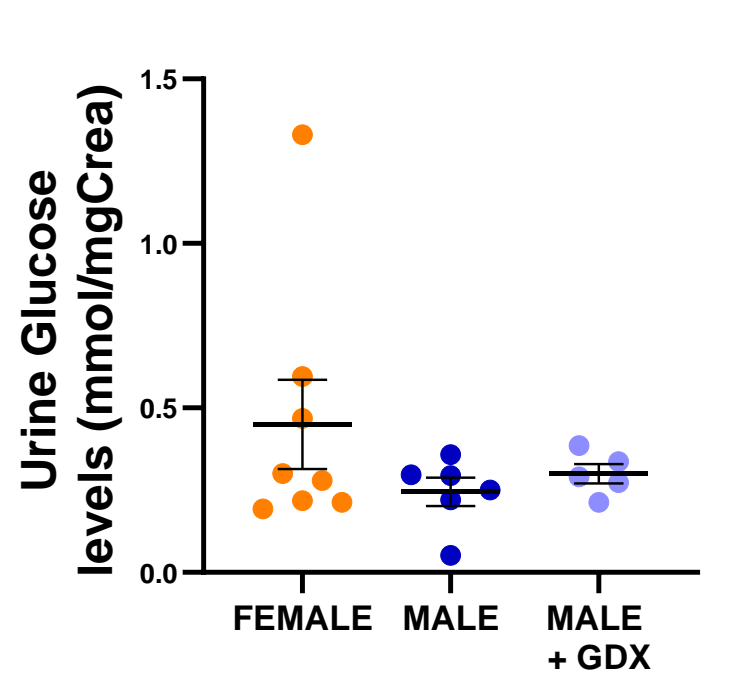

Metabolite measurement:

glucose, lactate,

glutamine, and glutamate

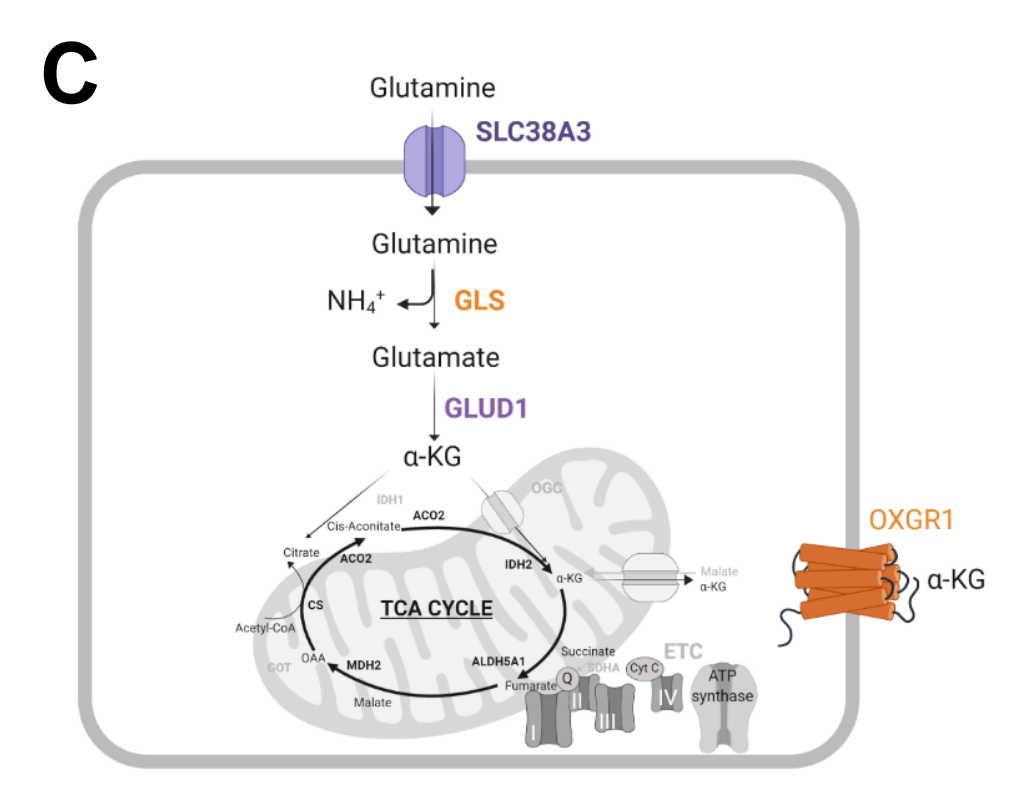

D

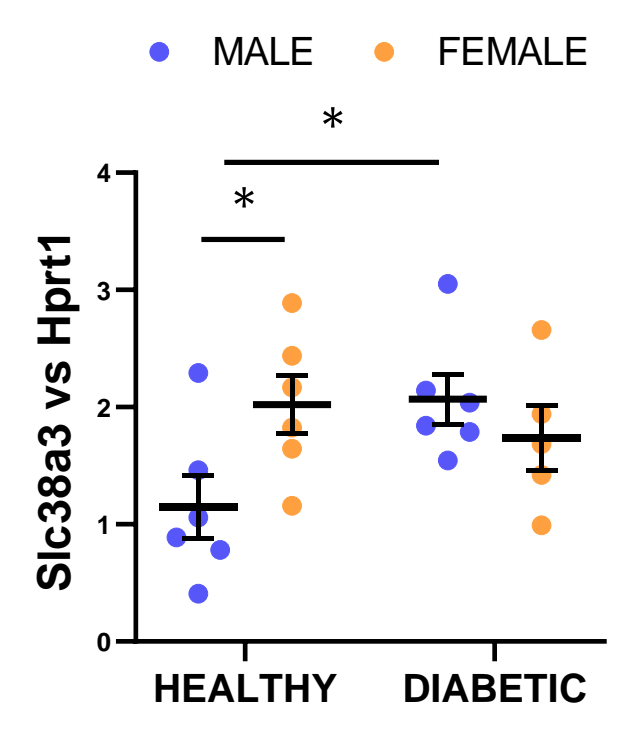

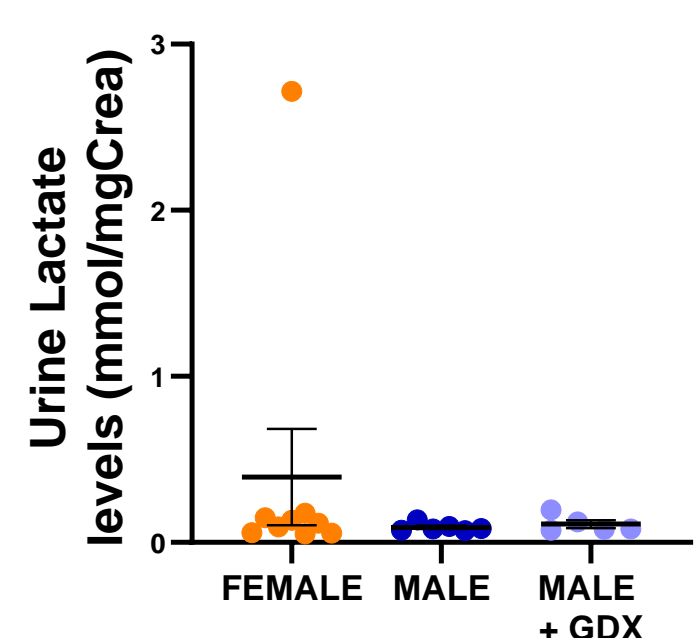

MALE
+ GDX

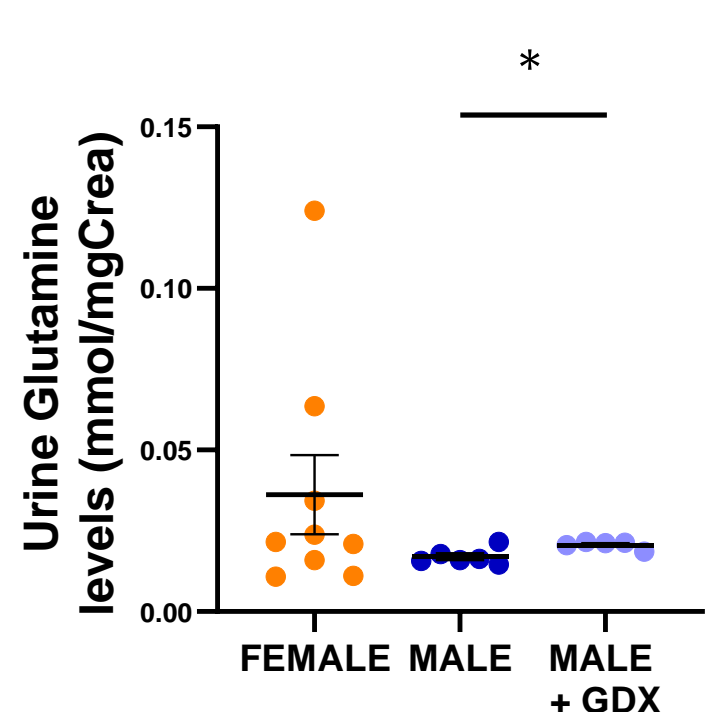

$+\mathrm{GDX}$
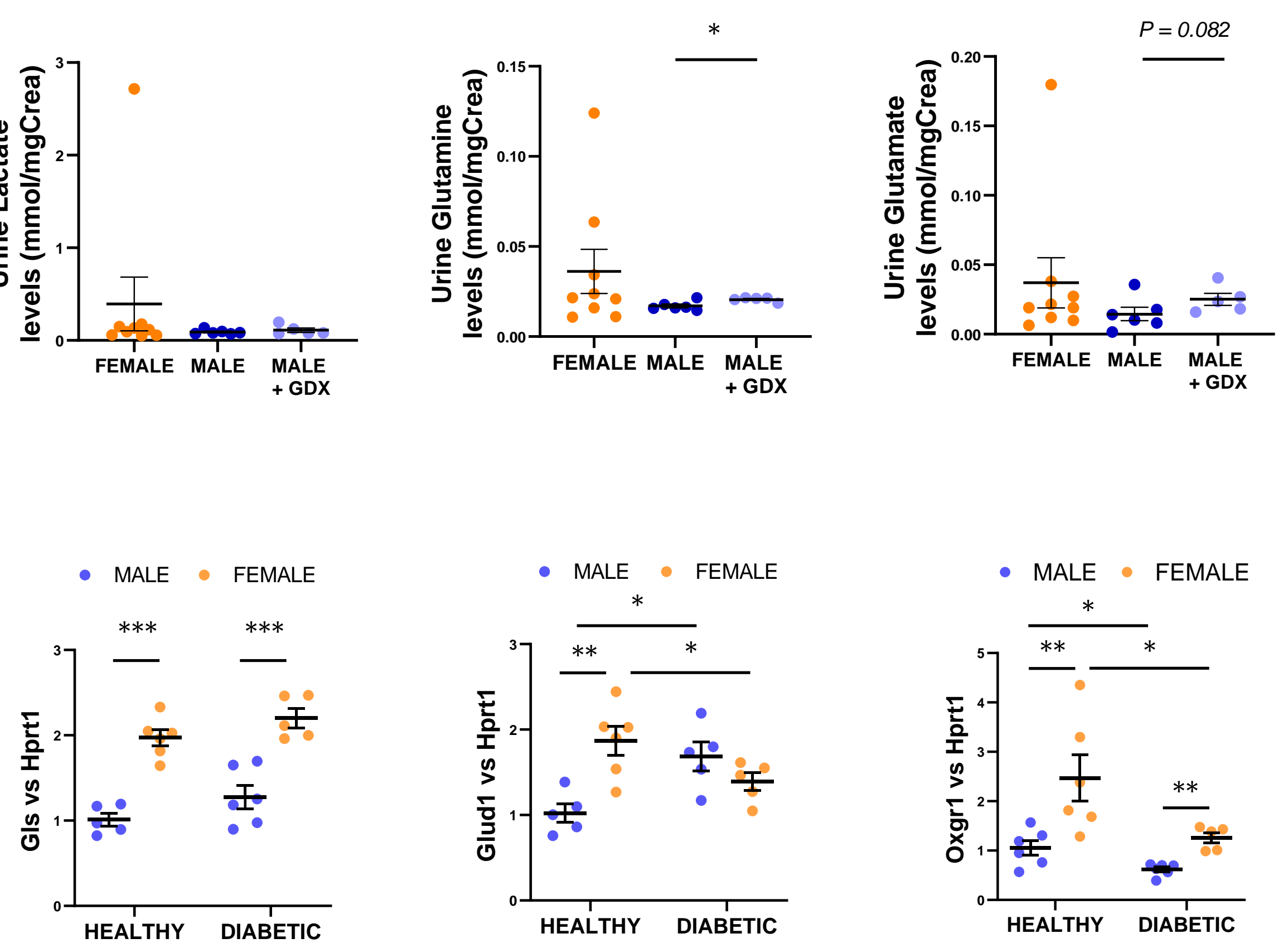
Figure S6. Representative correlations involving the circulating levels of $\gamma$-glutamylaminoacids, glucose, and glutamine in the human cohort. Pearson correlation was calculated for all possible pairs of circulating metabolites and correlation p-values were adjusted for multiple testing using the Benjamini-Hochberg method. The significance of each correlation was adjusted for an estimated 5\% FDR. The scattered dot plots in panel A display the significant positive correlation between the circulating levels of 4 representative $\gamma$-glutamylaminoacids altered by sex and/or diabetes, namely $\gamma$-glutamyl-alpha-lysine, $\gamma$-glutamylalanine, $\gamma$-glutamylglutamine, and $\gamma$ glutamylglutamate. The serum levels of $\gamma$-glutamyl-alpha-lysine, $\gamma$-glutamylalanine, and $\gamma$ glutamylglutamate did not correlate with the levels of glucose. Instead, these metabolites showed a significant negative correlation with the levels of glutamine, and a significant positive correlation with the levels of unconjugated glutamate $(B)$. 


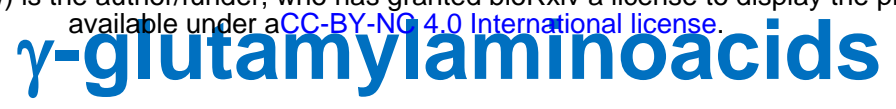
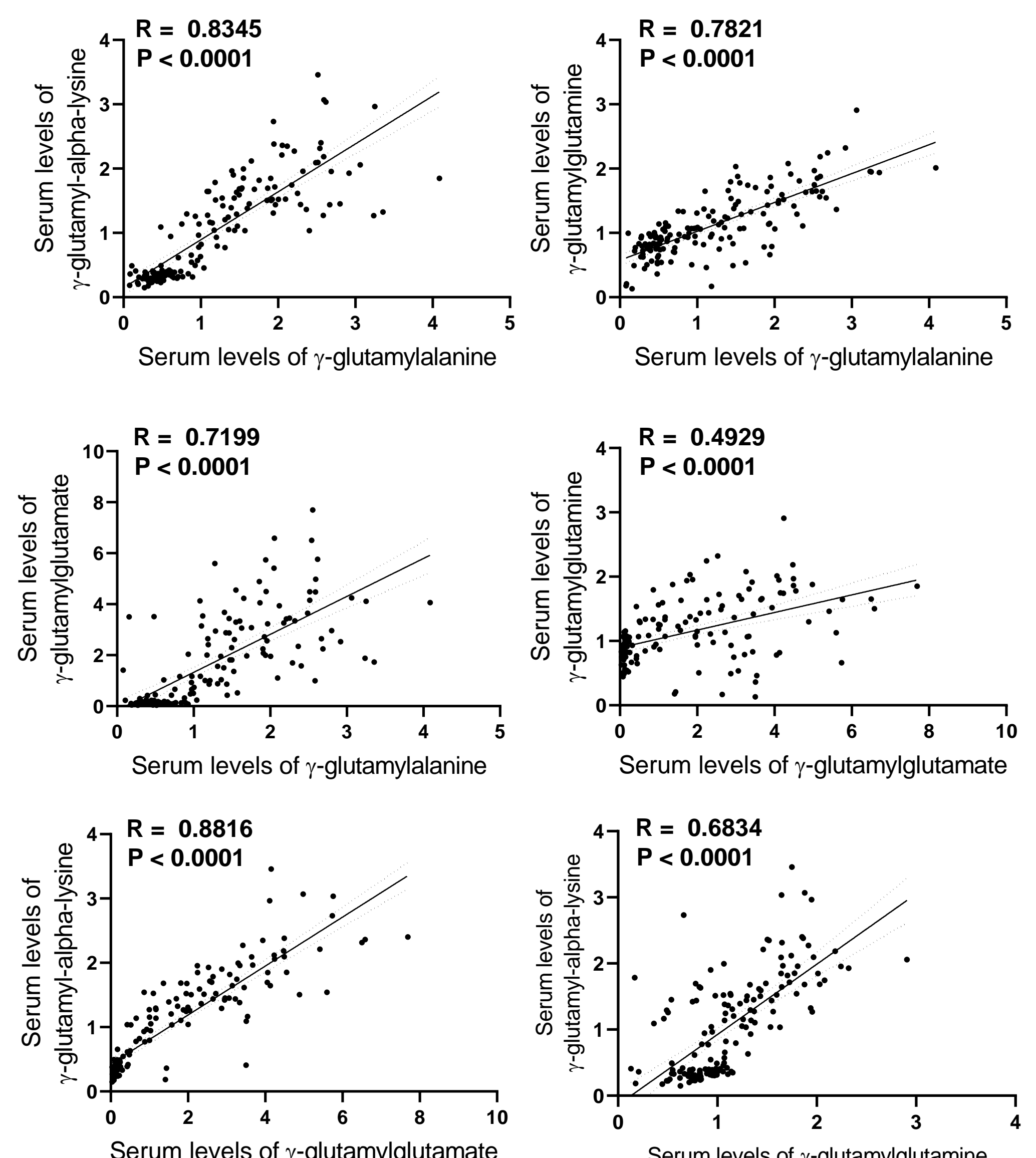

Serum levels of $\gamma$-glutamylglutamine
No correlation with serum levels of glucose
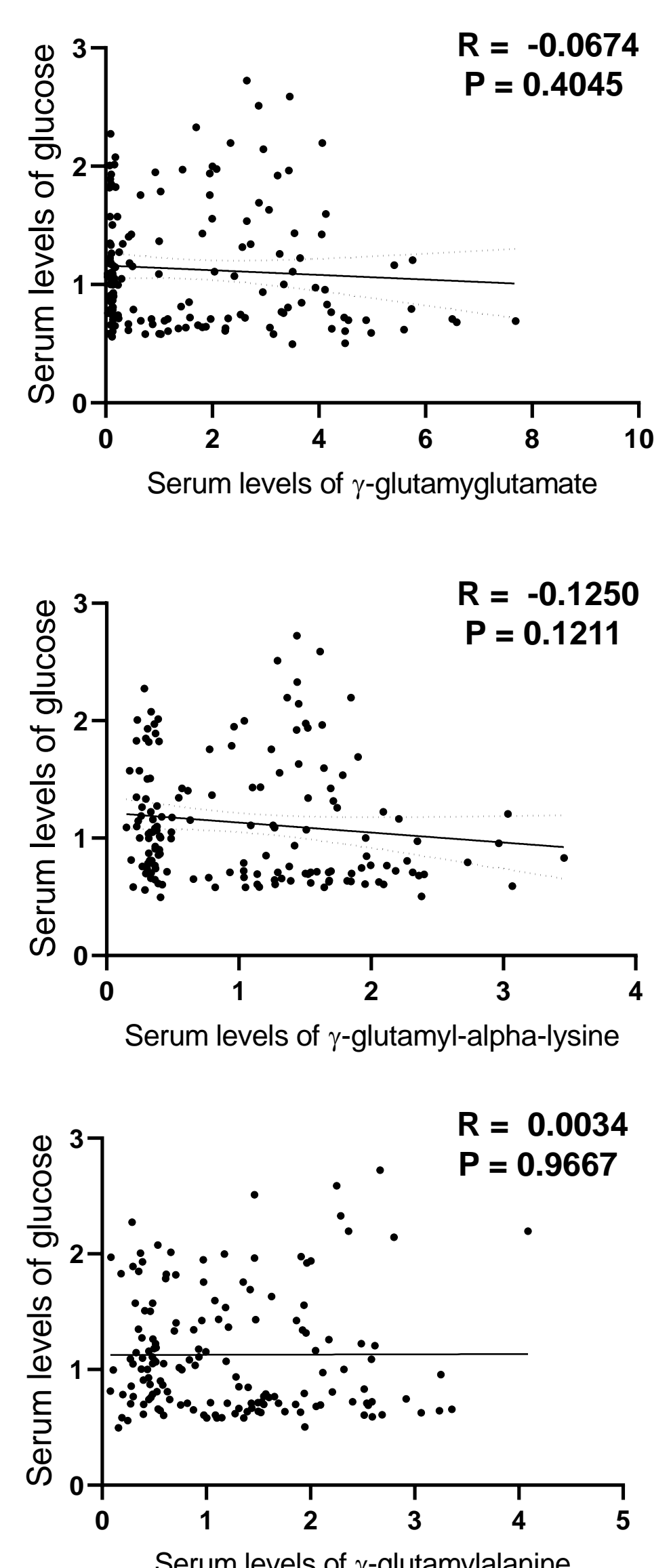

Negative correlation with serum levels of glutamine
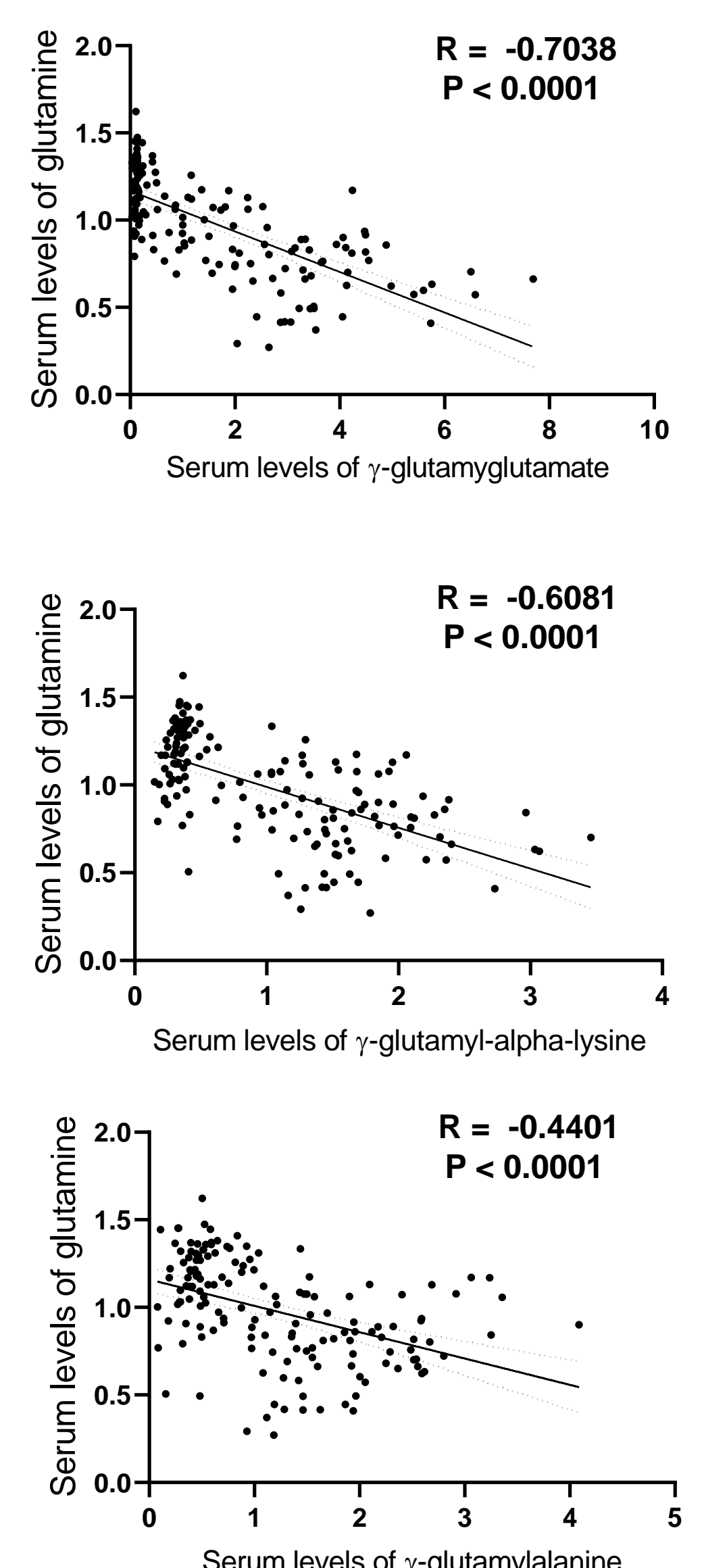

Positive correlation with serum levels of glutamate
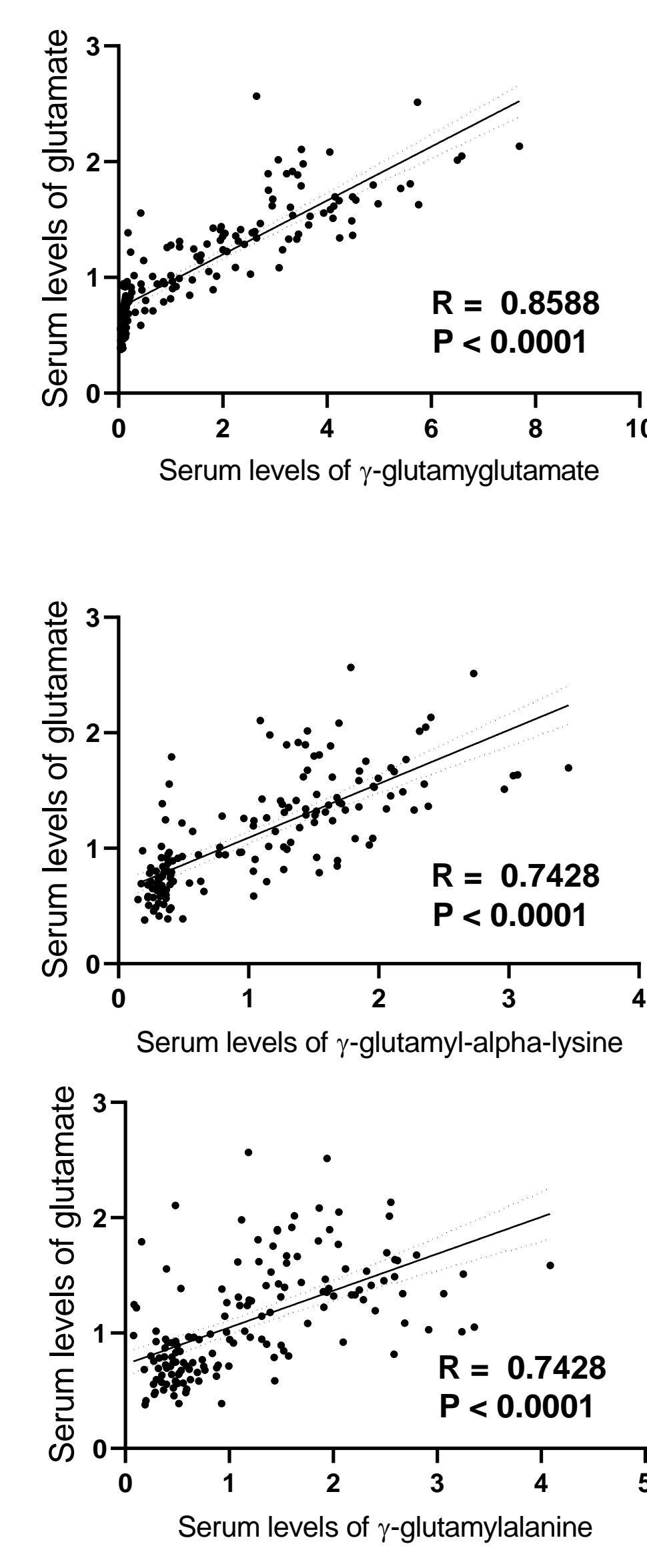
Figure S7. Identification of sex-biased genes and transcriptional regulators in the human

kidney. To identify transcriptional mechanisms linked to the effect of sex hormones in the kidney tubule, we studied the 60 proteins upregulated by DHT, as well as the 18 upregulated by EST, in PTECs. To identify genes differentially expressed between male and female kidneys that could relate to our findings in tubular cells we analyzed publicly available gene expression data in Nephroseq database. Four data sets of the human healthy kidney tubulointerstitium including information about the sex of the sample were identified: Woroniecka et al., Ju et al., Lindenmeyer et al., and the European Renal cDNA Bank (ERCB, unpublished). The Venn diagrams depict the overlap between genes that showed a sex-biased expression with $\mathrm{P}<0.05$ in each data set. Significantly altered genes showing the same direction of change in at least 2/4 data sets were retained. The analysis led to the identification of 196 genes significantly upregulated in the male tubulointerstitium and 139 genes significantly upregulated in the female tubulointerstitium. 


\section{Physiological sex differences}

in the human kidney

\section{Effect of Sex hormones}

Proteome data sets in human PTECs (Clotet et al., MCP, 2017)

60 Proteins regulated by

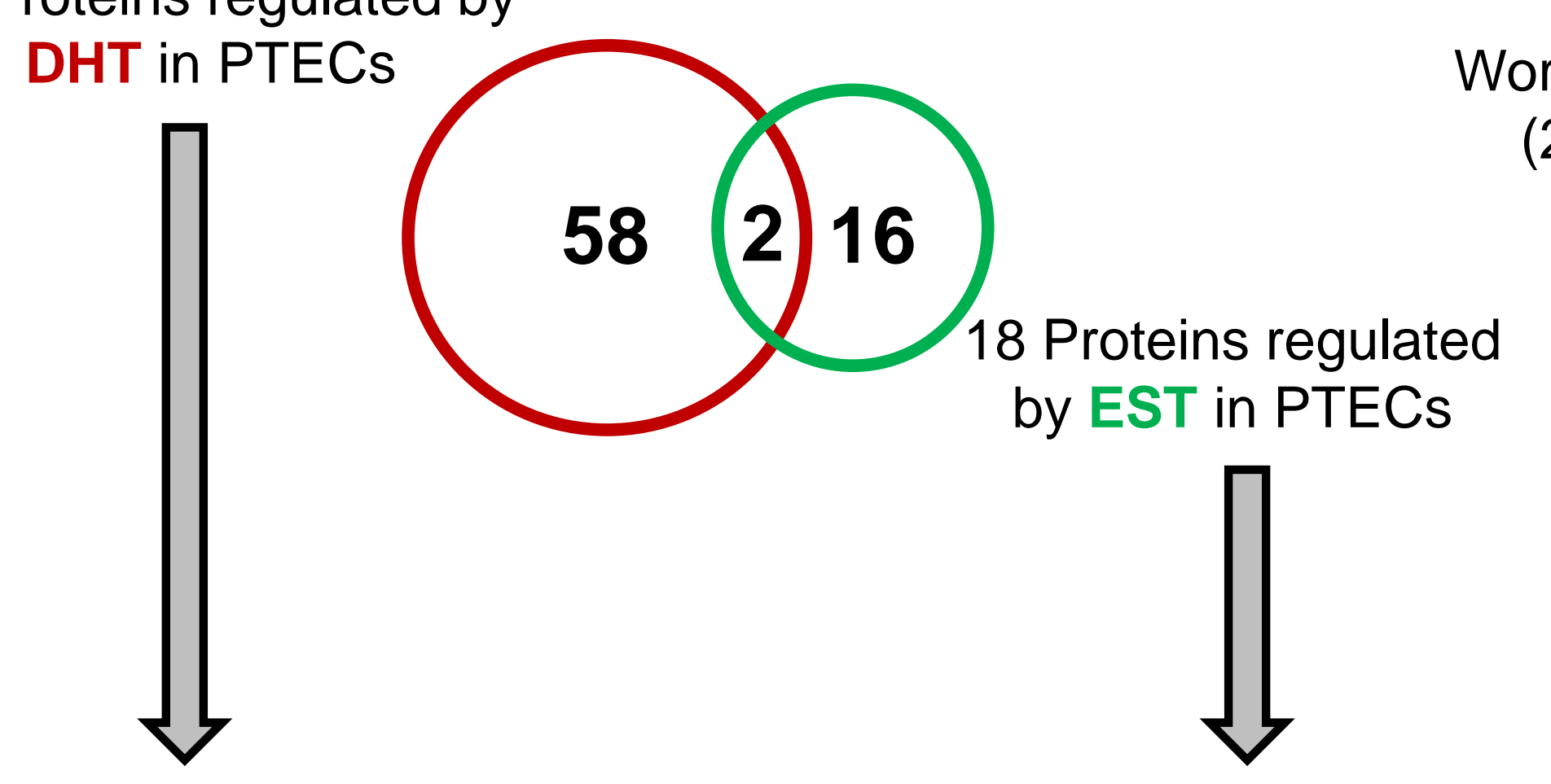

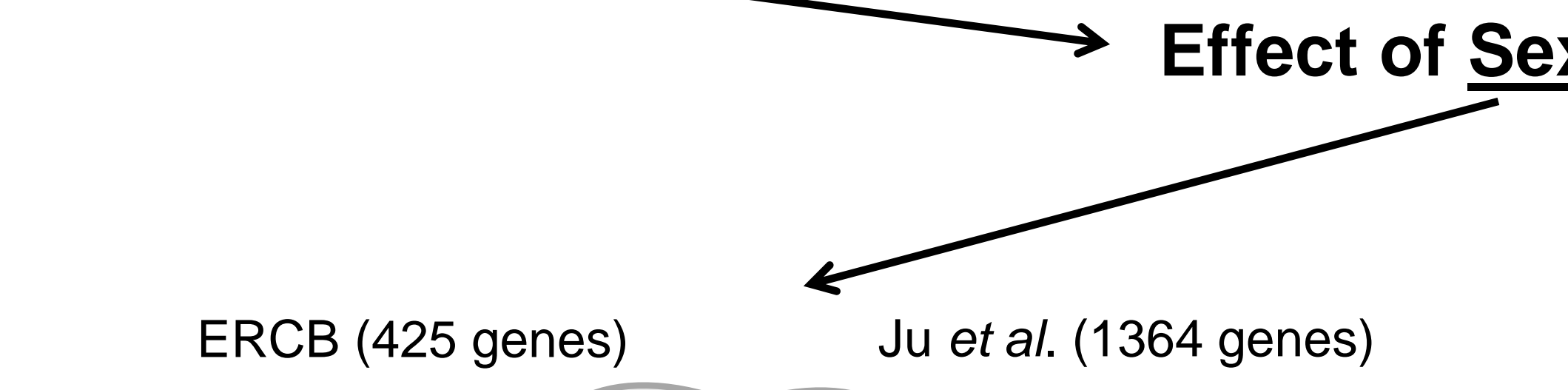$$
\text { ERCB (425 genes) }
$$$$
\text { Ju et al. (1364 genes) }
$$

Woroniecka et al. (242 genes)
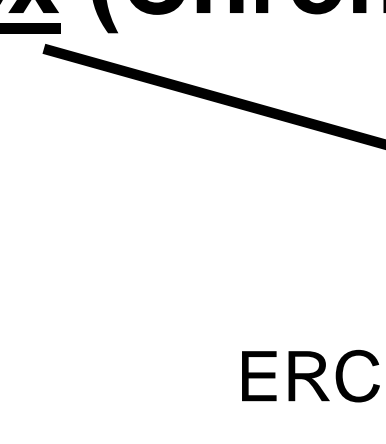

ERCB (240 genes)

Lidenmeyer et al.

Woroniecka et al. (562 genes)
Lidenmeyer et al.
Nephroseq database: 'Human Healthy

Tubulointerstitium' transcriptome data sets

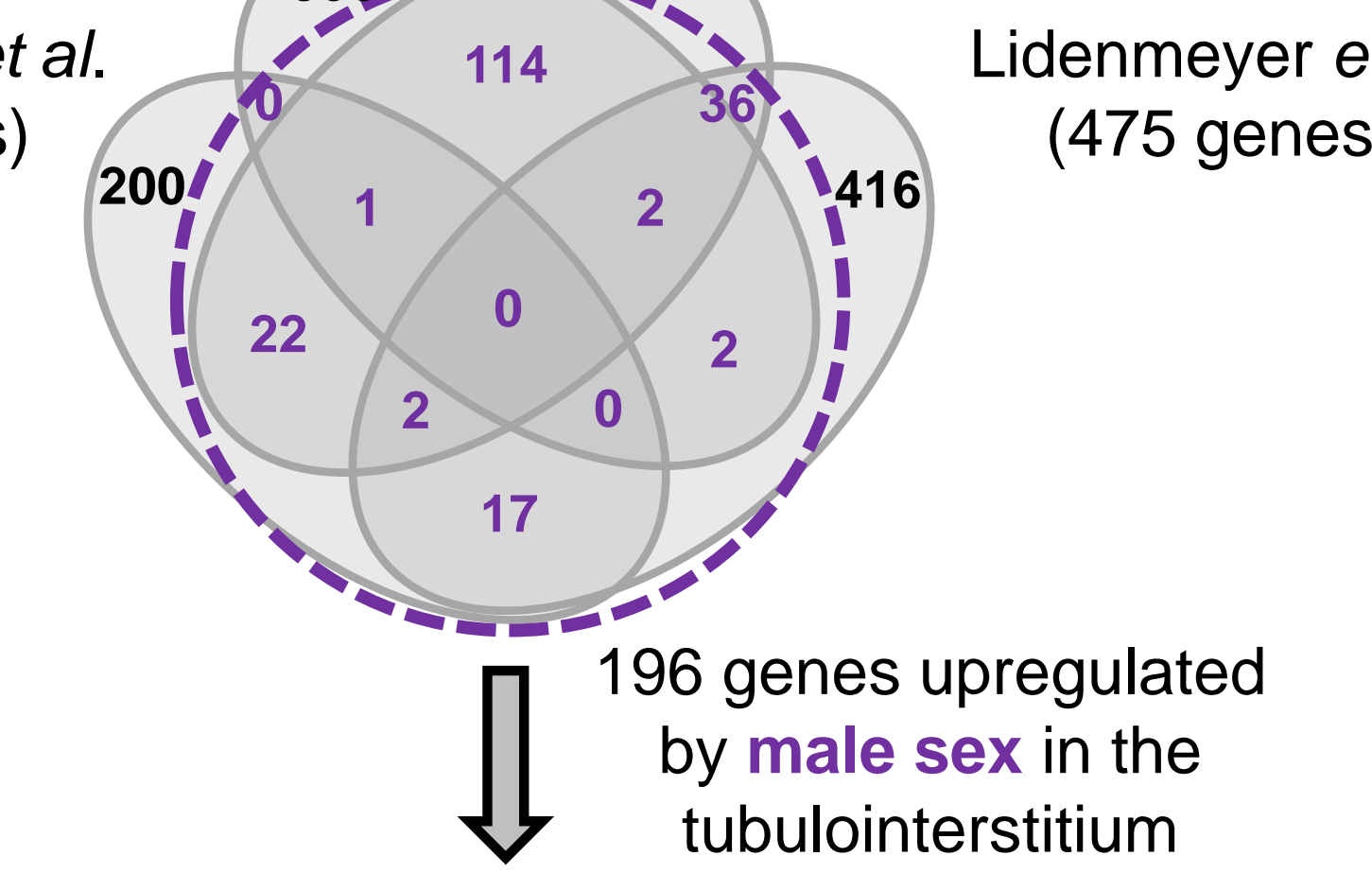

139 genes upregulate

tubulointersitium

tubulointerstitium 
Figure S8. Sex-biased expression of Y-linked and X-escape genes in PTECs. Male and female PTECs were exposed to ethanol (CONT), 100nM DHT or 100nM EST for 16h ( $\mathrm{n}=3 / \mathrm{sex}$; $n=4-6 /$ treatment). The gene expression of $\operatorname{KDM5D}(A), \operatorname{UTY}(B)$, EIF1AY (C), DDX3X (D), ZFX $(E)$, and EIF1AX (F) was measured and normalized to RPL31. Group-to-group differences were determined using pairwise $t$ tests for variables following a normal distribution, and Mann-Whitney tests for variables with a non-parametric distribution. ${ }^{*} p<0.05 ;{ }^{* *} p<0.01 ;{ }^{* *} p<0.001$. PTECs, proximal tubular epithelial cells; DHT, dihydrotestosterone; EST, estradiol. 
A

B

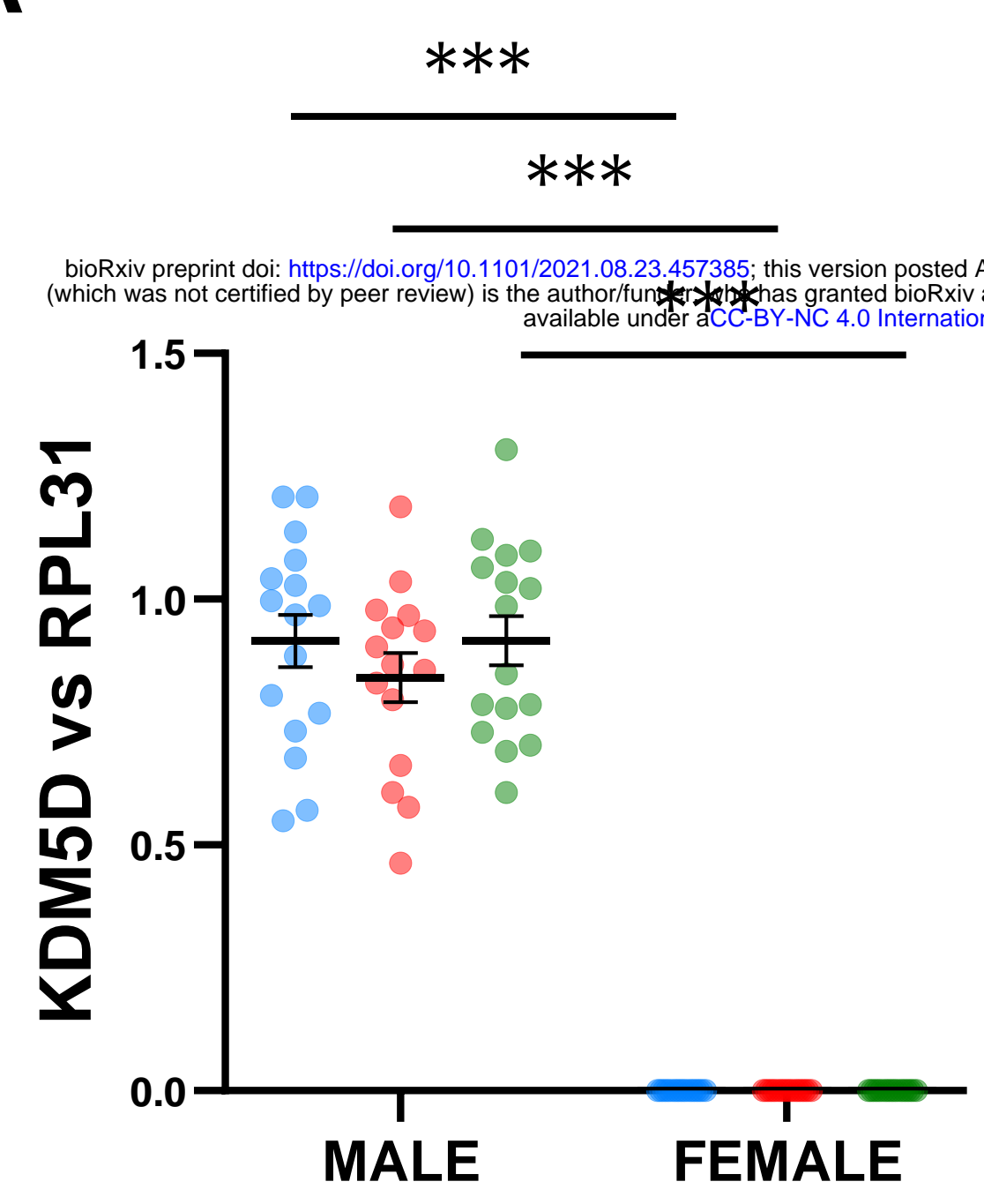

D

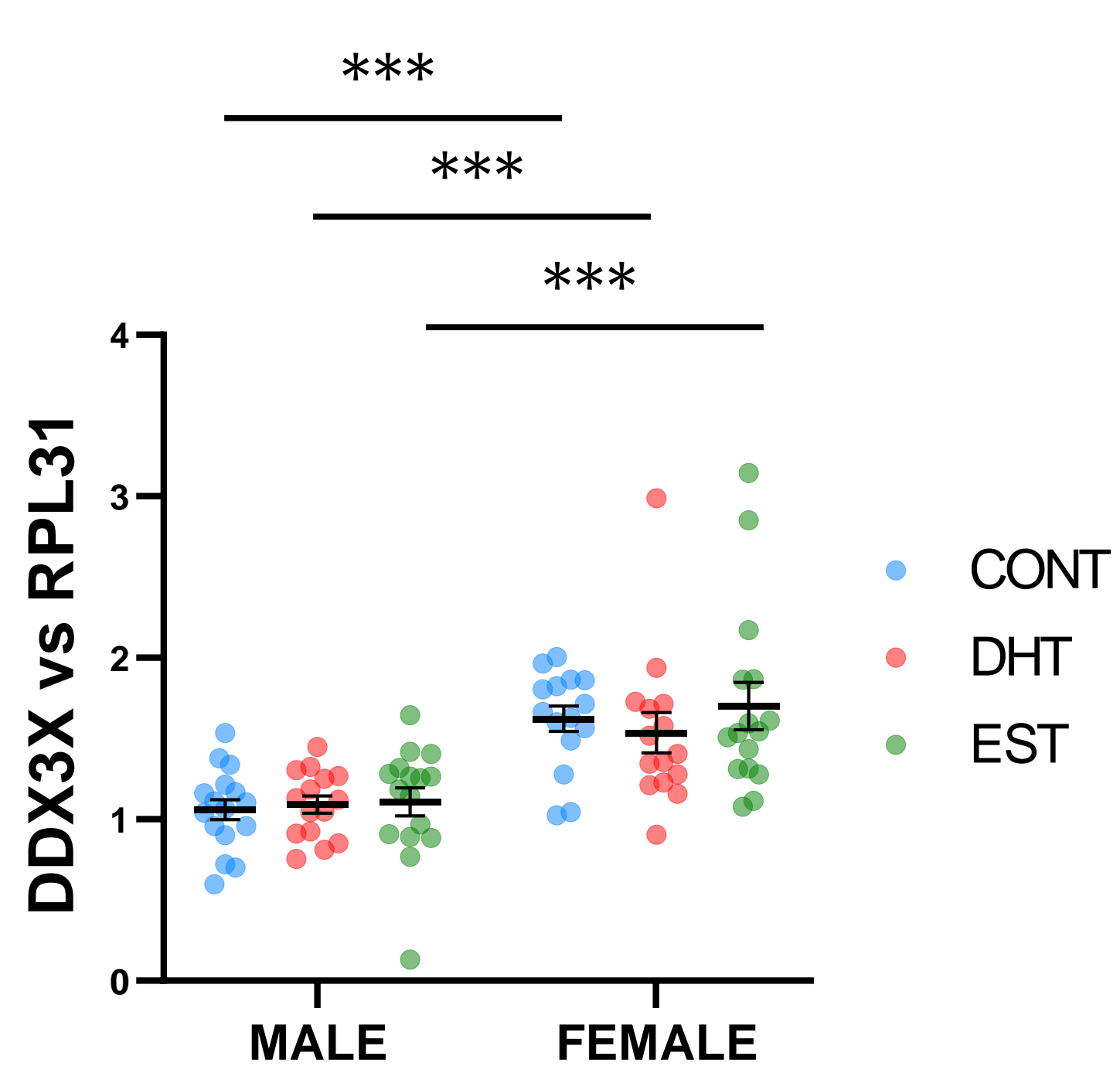

CONT

- DHT

- EST

E

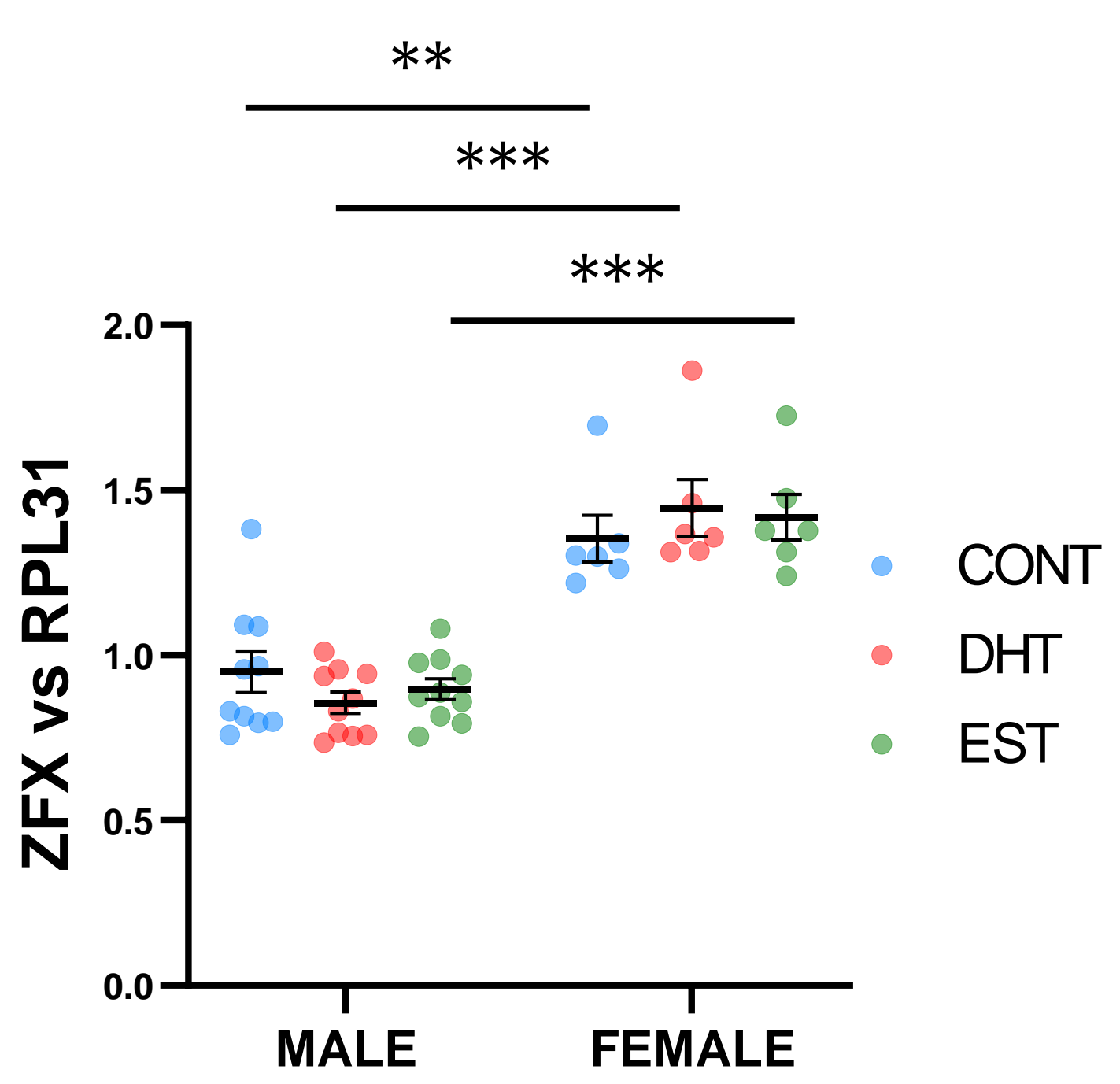

C

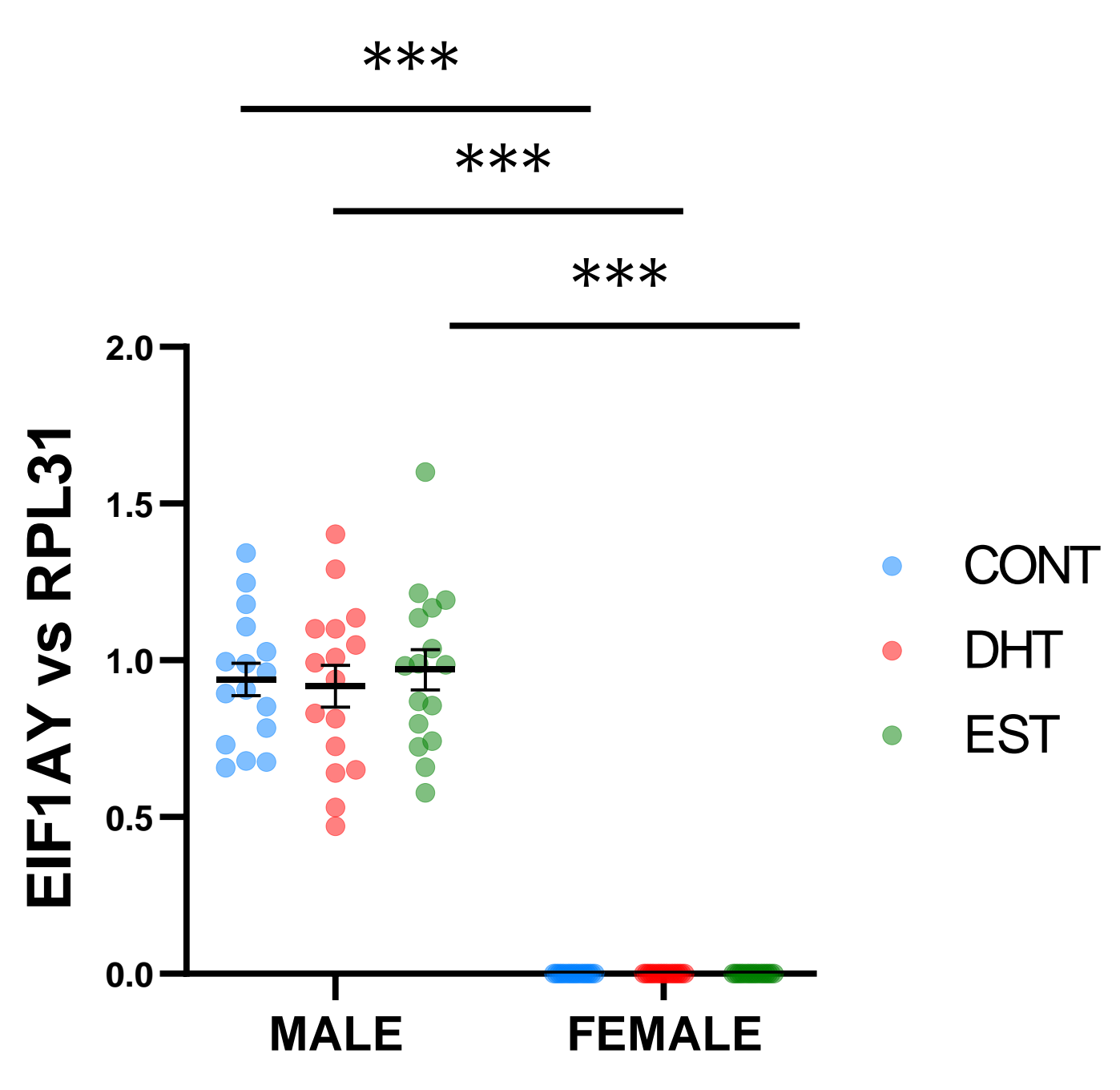

F

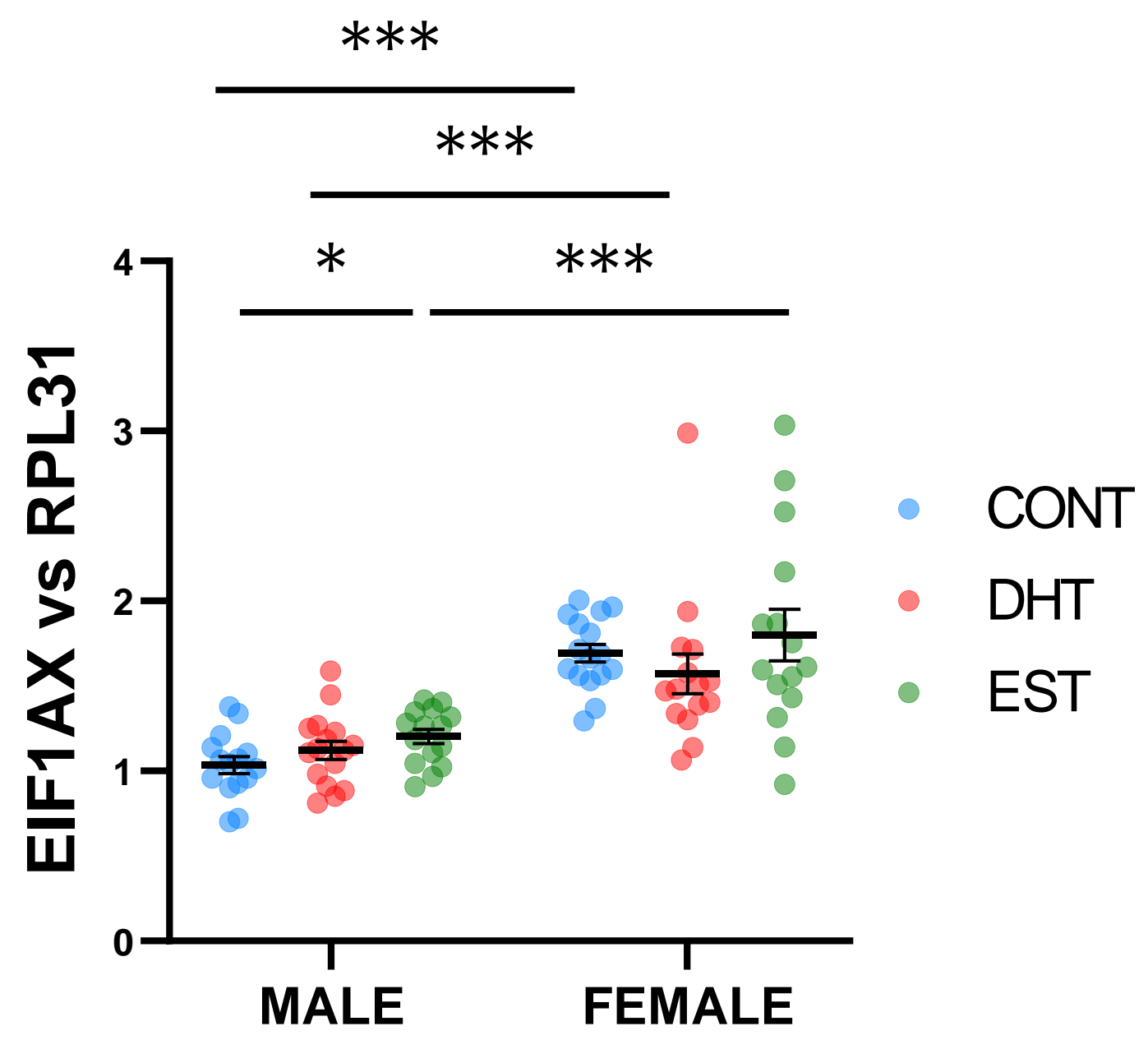


Figure S9. Serum and urine metabolome analysis in a subset of cases from the iCARE cohort. The flowchart illustrates the total number of cases encompassing the iCARE cohort, including non-diabetic males, non-diabetes females, males with T2DM, and females with T2DM. The subgroup of cases encompassing the metabolome study is also shown. T2DM, type 2 diabetes mellitus. 
